\title{
Shot Noise in Mesoscopic Conductors
}

\author{
Ya. M. Blanter and M. Büttiker \\ Département de Physique Théorique, Université de Genève, CH-1211, Genève 4, Switzerland
}

\begin{abstract}
Theoretical and experimental work concerned with dynamic fluctuations has developed into a very active and fascinating subfield of mesoscopic physics. We present a review of this development focusing on shot noise in small electric conductors. Shot noise is a consequence of the quantization of charge. It can be used to obtain information on a system which is not available through conductance measurements. In particular, shot noise experiments can determine the charge and statistics of the quasiparticles relevant for transport, and reveal information on the potential profile and internal energy scales of mesoscopic systems. Shot noise is generally more sensitive to the effects of electron-electron interactions than the average conductance. We present a discussion based on the conceptually transparent scattering approach and on the classical Langevin and BoltzmannLangevin methods; in addition a discussion of results which cannot be obtained by these methods is provided. We conclude the review by pointing out a number of unsolved problems and an outlook on the likely future development of the field.
\end{abstract}

(October 18, 1999)

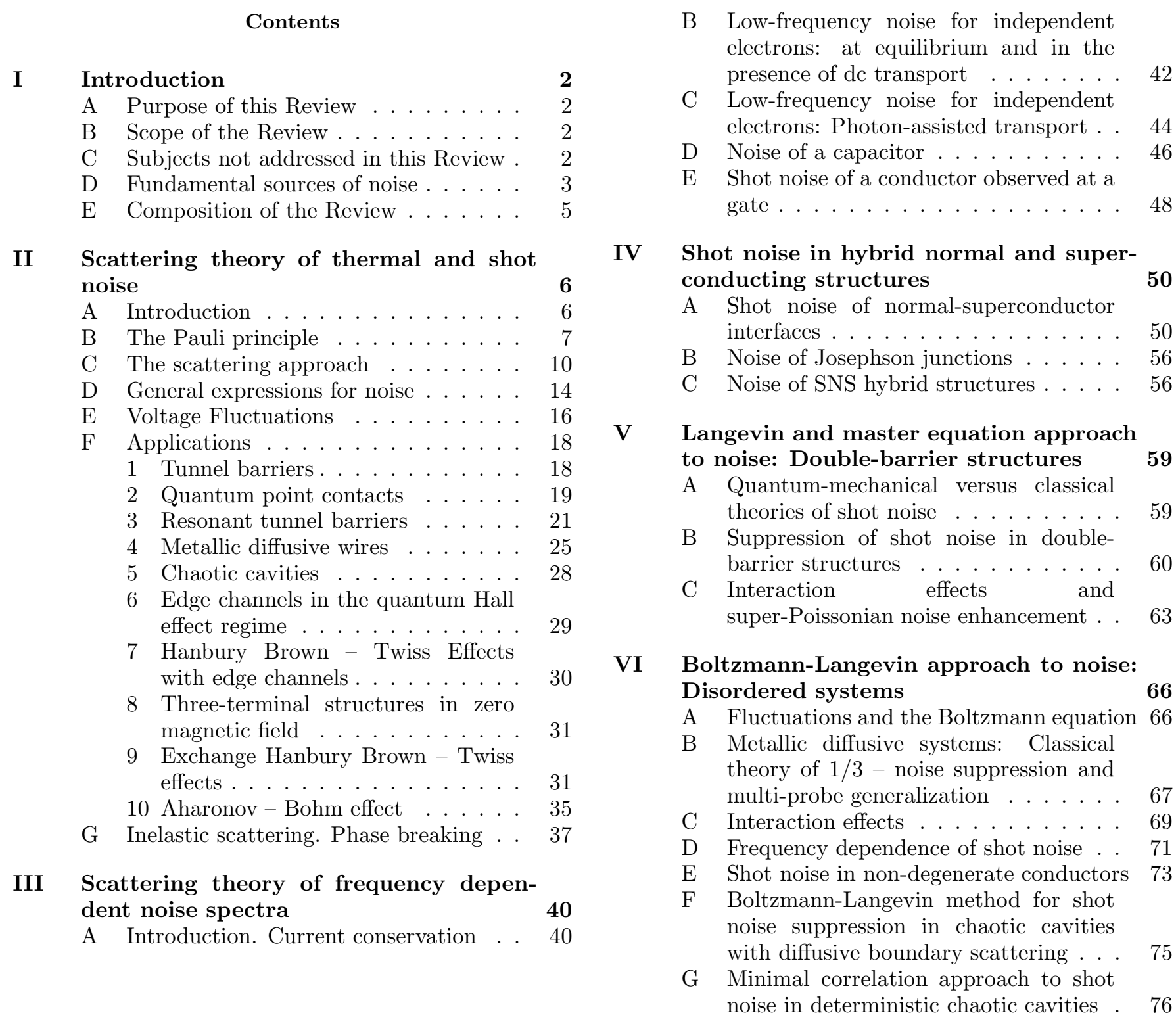


\begin{tabular}{|lll}
\hline VII & Noise in strongly correlated systems & $\mathbf{7 8}$
\end{tabular} A Coulomb blockade .......... . 78

B Anderson and Kondo impurities. . . . 83

C Tomonaga-Luttinger liquids and fractional quantum Hall edge states . . . . 84

D Composite fermions . . . . . . . 87

VIII Concluding remarks, future prospects, and unsolved problems 88

A General considerations ........ 88

B Summary for a lazy or impatient reader 89

\section{APPENDIXES}

90

A Counting statistics and optical analogies 90

B Spin effects and entanglement 92

C Noise induced by thermal transport

\section{INTRODUCTION}

\section{A. Purpose of this Review}

During the past two decades mesoscopic physics has developed into a fascinating subfield of condensed matter physics. In this article, we review a special topic of this field: We are concerned with the dynamical noise properties of mesoscopic conductors. After a modest start, a little more than a decade ago, both theories and experiments have matured. There is now already a substantial theoretical literature and there are a number of interesting experiments with which theoretical predictions can be compared. Some experiments ask for additional theoretical work. The field has thus reached a stage in which a review might be useful as a first orientation for researchers who wish to enter the field. Also researchers which are already active in the field might appreciate a review to help them keep an overview over the rapid development which has occurred. Any review, of course, reflects the authors' preferences and prejudices and in any case cannot replace the study of the original literature.

Presently, there are no reviews covering the actual state of development of the field. The only article which provides a considerable list of references, and gives a description of many essential features of shot noise in mesoscopic systems, has been written in 1996 by de Jong and Beenakker [1]. It is useful as a first introduction to the subject, but since then the field has developed considerably, and a broader review is clearly desirable. An additional brief review has been written by Martin [2]. The subject has been touched in books with broader scopes by Kogan [3] (Chapter 5) and Imry [4] (Chapter 8). These reviews, and in particular, the work of de Jong and Beenakker [1], provided a considerable help in starting this project.

\section{B. Scope of the Review}

Our intention is to present a review on shot noise in mesoscopic conductors. An effort is made to collect a complete list of references, and if not comprehensively re-derive, then at least to mention results relevant to the field. We do not cite conference proceedings and brief commentaries, unless we feel that they contain new results or bring some understanding which cannot be found elsewhere. Certainly, it is very possible that some papers, for various reasons, have not come to our attention. We apologize to the authors whose papers we might have overlooked.

Trying to classify the already large literature, we chose to divide the Review into sections according to the methods by which the results are derived and not according to the systems we describe. Many results can be obtained in the framework of the scattering approach and/or by classical methods. We deliberately avoid an explanation of the Green's function method, the master equation approach, and the bosonization technique. An attempt to explain how any one of these approaches work would probably double the size of this Review, which is already long enough. Consequently, we make an effort to re-derive the results existing in the literature by either the scattering or one of the classical (but not the master equation) approaches, and to present a unifying description. Certainly, for some systems these simple methods just do not work. In particular, this concerns Section VII, which describes shot noise in strongly correlated systems. Results obtained with more sophisticated methods are discussed only briefly and without an attempt to rederive them. We incorporate a number of original results in the text; they are usually extensions of results available in the literature, and are not specially marked as original.

A good review article should resemble a textbook useful to learn the subject but should also be a handbook for an expert. From this perspective this Review is more a textbook than a handbook. We encourage the reader who wants to enter the field to read the Review from the beginning to the end. On the other hand, we also try to help the experts, who only take this Review to look for the results concerning some particular phenomenon. It is for this reason that we have included the Table I with references to the different subsections in which the subject is discussed. The Review is concluded by a brief summary.

\section{Subjects not addressed in this Review}

First of all, we emphasize that this is not a general review of mesoscopic physics. In many cases, it is necessary to describe briefly the systems before addressing their noise properties. Only a few references are provided to the general physical background. These references are 
not systematic; we cite review articles where this is possible, and give references to the original papers only in cases such reviews are not readily available. This Review is not intended to be a tool to study mesoscopic physics, though possibly many important ideas are present here.

A multitude of sources typically contribute to the noise of electrical conductors. In particular, some of the early experimental efforts in this field suffered from $1 / f$-noise which is observed at low frequencies. There is still to our knowledge no established theory of $1 / f$-noise. The present article focuses on fundamental, unavoidable sources of noise, which are the thermal (NyquistJohnson-noise) and the shot noise due to the granularity of charge. The reader may find an account of other sources of noise in the book by Kogan [3].

We mention also that, though we try to be very accurate in references, this Review cannot be regarded as a historical chronicle of shot noise research. We re-derive a number of results by other methods than were used in the original papers, and even though we make an effort to mention all achievements, we do not emphasize historical priorities.

\section{Fundamental sources of noise}

Thermal noise. At non-zero temperature, thermal fluctuations are an unavoidable source of noise. Thermal agitation causes the occupation number of the states of a system to fluctuate.

Typically, in electric conductors, we can characterize the occupation of a state by an occupation number $n$ which is either zero or one. The thermodynamic average of the occupation number $\langle n\rangle$ is determined by the Fermi distribution function $f$ and we have simply $\langle n\rangle=f$. In an equilibrium state the probability that the state is empty is on the average given by $1-f$, and the probability that the state is occupied is on the average given by $f$. The fluctuations away from this average occupation number are $(n-\langle n\rangle)^{2}=n^{2}-2 n\langle n\rangle+\langle n\rangle^{2}$. Taking into account that for a Fermi system $n^{2}=n$, we find immediately that the fluctuations of the occupation number at equilibrium away from its thermal average are given by

$$
\left\langle(n-\langle n\rangle)^{2}\right\rangle=f(1-f) .
$$

The mean squared fluctuations vanish in the zero temperature limit. At high temperatures and high enough energies the Fermi distribution function is much smaller than one and thus the factor $1-f$ in Eq. (11) can be replaced by 1 . The fluctuations are determined by the (Maxwell) - Boltzmann distribution.

The fluctuations in the occupation number give rise to equilibrium current fluctuations in the external circuit which are via the fluctuation-dissipation theorem related to the conductance of the system. Thus investigation of equilibrium current fluctuations provides the same information as investigation of the conductance. This is not so with the shot noise of electrical conductors which provides information which cannot be obtained from a conductance measurement. We next briefly discuss the source of shot noise.

Shot noise. Shot noise in an electrical conductor is a consequence of the quantization of the charge. Unlike for thermal noise, to observe shot noise, we have to investigate the non-equilibrium (transport) state of the system.

To explain the origin of shot noise we consider first a fictitious experiment in which only one particle is incident upon a barrier. At the barrier the particle is either transmitted with probability $T$ or reflected with probability $R=1-T$. We now introduce the occupation numbers for this experiment. The incident state is characterized by an occupation number $n_{i n}$, the transmitted state is characterized by an occupation number $n_{T}$ and the reflected state by an occupation number $n_{R}$. If we repeat this experiment many times, we can investigate the average of these occupation numbers and their fluctuations away from the average behavior. In our experiments the incident beam is occupied with probability 1 and thus $\left\langle n_{i n}\right\rangle=1$. However the transmitted state is occupied only with probability $T$ and is empty with probability $R$. Thus $\left\langle n_{T}\right\rangle=T$ and $\left\langle n_{R}\right\rangle=R$. The fluctuations away from the average occupation number, can be obtained very easily in the following way: The mean squared fluctuations in the incident beam vanish, $\left(n_{i n}-\left\langle n_{i n}\right\rangle\right)^{2}=0$. To find the mean squared fluctuations in the transmitted and reflected state, we consider the average of the product of the occupation numbers of the transmitted and reflected beam $\left\langle n_{T} n_{R}\right\rangle$. Since in each event the particle is either transmitted or reflected, the product $n_{T} n_{R}$ vanishes for each experiment, and hence the average vanishes also, $\left\langle n_{T} n_{R}\right\rangle=0$. Using this we find easily that the mean squares of the transmitted and reflected beam and their correlation is given by

$$
\left\langle\left(\Delta n_{T}\right)^{2}\right\rangle=\left\langle\left(\Delta n_{R}\right)^{2}\right\rangle=-\left\langle\Delta n_{T} \Delta n_{R}\right\rangle=T R,
$$

where we have used the abbreviations $\Delta n_{T}=n_{T}-\left\langle n_{T}\right\rangle$ and $\Delta n_{R}=n_{R}-\left\langle n_{R}\right\rangle$. Such fluctuations are called partition noise since the scatterer divides the incident carrier stream into two streams. The partition noise vanishes both in the limit when the transmission probability is 1 and in the limit when the transmission probability vanishes, $T=0$. In these limiting cases no partitioning takes place. The partition noise is maximal if the transmission probability is $T=1 / 2$.

Let us next consider a slightly more sophisticated, but still fictitious, experiment. We assume that the incident beam is now occupied only with probability $f$. Eventually $f$ will just be taken to be the Fermi distribution function. The initial state is empty with probability $1-f$. Apparently, in this experiment the average incident occupation number is $\left\langle n_{i n}\right\rangle=f$, and since the particle is transmitted only with probability $f T$ and reflected with probability $f R$, we have $\left\langle n_{T}\right\rangle=f T$ and $\left\langle n_{R}\right\rangle=f R$. Since we are still only considering a single particle, we 
have as before that in each event the product $n_{T} n_{R}$ vanishes. Thus we can repeat the above calculation to find

$$
\begin{gathered}
\left\langle\left(\Delta n_{T}\right)^{2}\right\rangle=T f(1-T f), \\
\left\langle\left(\Delta n_{R}\right)^{2}\right\rangle=R f(1-R f), \\
\left\langle\Delta n_{T} \Delta n_{R}\right\rangle=-T R f^{2} .
\end{gathered}
$$

If we are in the zero temperature limit, $f=1$, we recover the results discussed above. Note that now even in the limit $T=1$ the fluctuations in the transmitted state do not vanish, but fluctuate like the incident state. For the transmitted stream, the factor $(1-T f)$ can be replaced by 1 , if either the transmission probability is small or if the occupation probability of the incident carrier stream is small.

We can relate the above results to the fluctuations of the current in a conductor. To do this we have to put aside the fact that in a conductor we deal not as above just with events of a single carrier but with a state which may involve many (indistinguishable) carriers. We imagine a perfect conductor which guides the incident carriers to the barrier, and imagine that we have two additional conductors which guide the transmitted and reflected carriers away from the conductor, such that we can discuss, as before, incident, transmitted and reflected currents separately. Furthermore, we want to assume that we have to consider only carriers moving in one direction with a velocity $v(E)$ which is uniquely determined by the energy $E$ of the carrier. Consider next the average incident current. In a narrow energy interval $d E$, the incident current is $d I_{\text {in }}(E)=e v(E) d \rho(E)$, where $d \rho(E)$ is the density of carriers per unit length in this energy range. The density in an energy interval $d E$ is determined by the density of states (per unit length) $\nu(E)=d \rho / d E$ times the occupation factor $n_{i n}(E)$ of the state at energy $E$. We thus have $d \rho(E)=n_{\text {in }}(E) \nu(E) d E$. The density of states in our perfect conductors is $\nu(E)=1 /(2 \pi \hbar v(E))$. Thus the incident current in a narrow energy interval is simply

$$
d I_{i n}(E)=\frac{e}{2 \pi \hbar} n_{i n}(E) d E .
$$

This result shows that there is a direct link between currents and the occupation numbers. The total incident current is $I_{i n}=(e / 2 \pi \hbar) \int n_{i n}(E) d E$ and on the average is given by $\left\langle I_{i n}\right\rangle=(e / 2 \pi \hbar) \int f(E) d E$. Similar considerations give for the average transmitted current $\left\langle I_{T}\right\rangle=(e / 2 \pi \hbar) \int f(E) T d E$ and for the reflected current $\left\langle I_{R}\right\rangle=(e / 2 \pi \hbar) \int f(E) R d E$. Current fluctuations are dynamic phenomena. The importance of the above consideration is that it can now easily be applied to investigate time-dependent current fluctuations. For occupation numbers which vary slowly in time, Eq. (6) still holds. The current fluctuations in a narrow energy interval are at long times determined by $d I_{i n}(E, t)=$ $(e / 2 \pi \hbar) n_{i n}(E, t) d E$ where $n_{\text {in }}(E, t)$ is the time dependent occupation number of states with energy $E$. A detailed derivation of the connection between currents and occupation numbers is the subject of an entire Section of this Review. We are interested in the low frequency current noise and thus we can Fourier transform this equation. In the low frequency limit we obtain $I(\omega)=(e / 2 \pi \hbar) \int d E n(E, E+\hbar \omega)$. As a consequence the fluctuations in current and the fluctuations in occupation number are directly related. In the zero frequency limit the current noise power is $S_{I I}=e^{2} \int d E S_{n n}(E)$. In each small energy interval particles arrive at a rate $d E /(2 \pi \hbar)$ and contribute, with a mean square fluctuation, as given by one of the equations (3) - (5), to the noise power. We have $S_{n n}(E)=(1 / \pi \hbar)\langle\Delta n \Delta n\rangle$. Thus the fluctuation spectra of the incident, transmitted, and reflected currents are

$$
\begin{gathered}
S_{I_{i n} I_{i n}}=2 \frac{e^{2}}{2 \pi \hbar} \int d E f(1-f) ; \\
S_{I_{T} I_{T}}=2 \frac{e^{2}}{2 \pi \hbar} \int d E T f(1-T f) ; \\
S_{I_{R} I_{R}}=2 \frac{e^{2}}{2 \pi \hbar} \int d E R f(1-R f) .
\end{gathered}
$$

The transmitted and reflected current are correlated,

$$
S_{I_{T} I_{R}}=-2 \frac{e^{2}}{2 \pi \hbar} \int d E T f R f
$$

In the limit that either $T$ is very small or $f$ is small, the factor $(1-T f)$ in Eq. (8) can be replaced by one. In this limit, since the average current through the barrier is $\langle I\rangle=(e / 2 \pi \hbar) \int d E T f$, the spectrum, Eq. (8), is Schottky's result [5] for shot noise,

$$
S_{I_{T} I_{T}}=2 e\langle I\rangle .
$$

Schottky's result corresponds to the uncorrelated arrival of particles with a distribution function of time intervals between arrival times which is Poissonian, $P(\Delta t)=$ $\tau^{-1} \exp (-\Delta t / \tau)$, with $\tau$ being the mean time interval between carriers. Alternatively, Eq. (11) is also referred to in the literature as the Poisson value of shot noise.

The result Eq. (8) is markedly different from Eq. (11) since it contains, in comparison to Schottky's expression, the extra factor $(1-T f)$. This factor has the consequence that the shot noise (8) is always smaller than the Poisson value. For truly ballistic systems $(T=1)$ the shot noise even vanishes in the zero temperature limit. As the temperature increases, in such a conductor $(T=1)$ there is shot noise due to the fluctuation in the incident beam arising from the thermal fluctuations. Eventually at high temperatures the factor $1-f$ can be replaced by 1 , and the ballistic conductor exhibits Poisson noise, in 
accordance with Schottky's formula, Eq. (11). The full Poisson noise given by Schottky's formula is also reached for a scatterer with very small transparency $T \ll 1$. We emphasize that the above statements refer to the transmitted current. In the limit $T \ll 1$ the reflected current remains nearly noiseless up to high temperatures when $(1-R f)$ can be replaced by 1 . We also remark that even though electron motion in vacuum tubes (the Schottky problem (5) is often referred to as ballistic, it is in fact a problem in which carriers have been emitted by a source into vacuum either through thermal activation over or by tunneling through a barrier with very small transparency.

Our discussion makes it clear that out of equilibrium, and at finite temperatures, the noise described by Eq. (8) contains the effect of both the fluctuations in the incident carrier beam as well as the partition noise. In a transport state, noise in mesoscopic conductors has two distinct sources which manifest themselves in the fluctuations of the occupation numbers of states: (i) thermal fluctuations; (ii) partition noise due to the discrete nature of carriers].

Both the thermal and shot noise at low frequencies and low voltages reflect in many situations independent quasi-particle transport. Electrons are, however, interacting entities and both the fluctuations at finite frequencies and the fluctuation properties far from equilibrium require in general a discussion of the role of the longrange Coulomb interaction. A quasi-particle picture is no longer sufficient and collective properties of the electron system come into play.

The above considerations are, of course, rather simplistic and should not be considered as a quantitative theory. Since statistical effects play a role, one would like to see a derivation which relates the noise to the symmetry of the wave functions. Since we deal with many indistinguishable particles, exchange effects can come into play. The following Sections will treat these questions in detail.

\section{E. Composition of the Review}

The review starts with a discussion of the scattering approach (Section [1]). This is a fully quantummechanical theory which applies to phase-coherent transport. It is useful to take this approach as a starting point because of its conceptual clarity. The discussion proceeds with a number of specific examples, like quantum point contacts, resonant double barriers, metallic diffu-

\footnotetext{
${ }^{1}$ Note that this terminology, common in mesoscopic physics, is different from that used in the older literature [6], where shot noise (due to random injection of particles into a system) and partition noise (due to random division of the particle stream between different electrodes, or by potential barriers) are two distinct independent sources of fluctuations.
}

sive wires, chaotic cavities, and quantum Hall conductors. We are interested not only in current fluctuations at one contact of a mesoscopic sample but also in the correlations of currents at different contacts. Predictions and experiments on such correlations are particularly interesting since current correlations are sensitive to the statistical properties of the system. Comparison of such experiments with optical analogs is particularly instructive.

Section III describes the frequency dependence of noise via the scattering approach. The main complication is that generally one has to include electron-electron interactions to obtain fluctuation spectra which are current conserving. For this reason, not many results are currently available on frequency-dependent noise, though the possibility to probe in this way the inner energy scales and collective response times of the system looks very promising.

We proceed in Section IV with the description of superconducting and hybrid structures, to which a generalized scattering approach can be applied. New noise features appear from the fact that the Cooper pairs in superconductors have the charge $2 e$.

Then in Sections $\mathrm{V}$ and $\mathrm{VI}$ we review recent discussions which apply more traditional classical approaches to the fluctuations of currents in mesoscopic conductors. Specifically, for a number of systems, as far as one is concerned only with ensemble averaged quantities, the Langevin and Boltzmann-Langevin approaches provide a useful discussion, especially since it is known how to include inelastic scattering and effects of interactions.

Section VII is devoted to shot noise in strongly correlated systems. This Section differs in many respects from the rest of the Review, mainly because strongly correlated systems are mostly too complicated to be described by the scattering or Langevin approaches. We resort to a brief description and commentary of the results, rather than to a comprehensive demonstration how they are derived.

\begin{tabular}{|c|c|}
\hline Subject & Subdivision \\
\hline \multicolumn{2}{|l|}{ Ballistic conductors } \\
\hline - Electron-phonon interactions & $\mathrm{VIO}$ \\
\hline $\begin{array}{l}\text { - Electron-electron interactions in } \\
\text { non-degenerate ballistic conductors }\end{array}$ & VIE \\
\hline - Hanbury Brown - Twiss effects & II F 8, IIF 9 \\
\hline & IV A, B \\
\hline - Aharonov-Bohm effect & II F 10 \\
\hline \multicolumn{2}{|l|}{ Tunnel barriers } \\
\hline - Normal barriers & II F 1 \\
\hline - Barriers in diffusive conductors & II F 4 \\
\hline - Coulomb blockade regime & VII A \\
\hline - Frequency dependence of noise & III B, III C \\
\hline - Barriers of oscillating random height & III O \\
\hline - NS interfaces & IV A \\
\hline • Josephson junctions & IV B \\
\hline $\begin{array}{l}\text { - Barriers in Luttinger liquids } \\
\text { - Counting statistics for normal }\end{array}$ & $\mathrm{VIIC}$ \\
\hline
\end{tabular}


and NS barriers

Quantum point contacts

- Normal quantum point contacts

- SNS contacts

Double-barrier structures

- Resonant tunneling; linear regime

- Double-barrier suppression

- Counting statistics

- Double wells and crossover to the diffusive regime

- Quantum wells in the non-linear regime: super-Poissonian shot noise enhancement

- Interaction effects in quantum wells

- Coulomb blockade in quantum dots (normal, superconducting, or ferromagnetic electrodes)

- Frequency dependence of noise

- Resonant tunneling through localized states; Anderson and Kondo models

- NINIS junctions

Disordered conductors

- Noise suppression in metallic diffusive wires

- Counting statistics

- Multi-terminal generalization and

Hanbury Brown - Twiss effects

- Interaction effects

- Frequency dependence of noise

- Disordered contacts and interfaces

- Disordered NS and SNS contacts

- Crossover to the ballistic regime

- Localized regime

- Non-degenerate diffusive conductors

- Composite fermions with disorder

- Noise induced by thermal transport

Chaotic cavities

- Noise suppression in two-terminal chaotic cavities

- Cavities with diffusive boundary scattering

- Multi-terminal generalization and

Hanbury Brown - Twiss effects

- Counting statistics

Quantum Hall effect

- IQHE edge channels

- Hanbury Brown - Twiss effects with

IQHE edge channels

- FQHE edge channels

- Composite fermions

Systems with purely capacitive coupling

- Frequency dependence of noise

TABLE I. The results reviewed in this article arranged by subject.
A

IIF 2

IV C

IIF 3

IIF 3, $\mathrm{VB}$

A

IIF 3, $\mathrm{VB}$

$\mathrm{NC}$

II G, $\mathrm{VB}$.

\section{A \\ $\mathrm{VB}, \mathrm{VO}$, \\ VII A}

IIF 3, VII B IV A

IIF 4, VIB

A

IIF 9, NIB

II G, VI d

IIIB, VID

IIF 4

IVA, IV O

IIF 4

IIF 4

VIE

VIID

d

IIF 5, $\mathrm{VIF}$,

VI G

VIF

II F 5, VIG

A

IIF 6, IIF 10

IIF 7, II F 9

VIIC

VIID

IIID III E

subject.

We conclude the Review (Section VIII) with an outlook. We give our opinion concerning possible future directions along which the research on shot noise will develop. In the concluding Section, we provide a very concise summary of the state of the field and we list some (possibly) important unsolved problems.

Some topics are treated in a number of Appendices mostly to provide a better organization of the manuscript. The Appendices report important results on topics which are relatively well rounded, are relevant for the connections between different sub-fields, and might very well become the subject of much further research.

\section{SCATTERING THEORY OF THERMAL AND SHOT NOISE}

\section{A. Introduction}

In this Section we present a theory of thermal and shot noise for fully phase-coherent mesoscopic conductors. The discussion is based on the scattering approach to electrical conductance. This approach, as we will show, is conceptually simple and transparent. A phasecoherent description is needed if we consider an individual sample, like an Aharonov-Bohm ring, or a quantum point contact. Often, however, we are interested in characterizing not a single sample but rather an ensemble of samples in which individual members differ only in the microscopic arrangement of impurities or small variations in the shape. The ensemble averaged conductance is typically, up to a small correction, determined by a classical expression like a Drude conductance formula. Similarly, noise spectra, after ensemble averaging, are, up to small corrections, determined by purely classical expressions. In these case, there is no need to keep information about phases of wavefunctions, and shot noise expressions may be obtained by classical methods. Nevertheless, the generality of the scattering approach and its conceptual clarity, make it the desired starting point of a discussion of noise in electrical conductors.

Below we emphasize a discussion based on second quantization. This permits a concise treatment of the many particle problem. Rather than introducing the Pauli principle by hand, in this approach it is a consequence of the underlying symmetry of the wave functions. It lends itself to a discussion of the effects related to the quantum mechanical indistinguishability of identical particles. In fact it is an interesting question to what extend we can directly probe the fact that exchange of particles leaves the wave function invariant up to a sign. Thus an important part of our discussion will focus on exchange effects in current-current correlation spectra.

We start this Section with a review of fluctuations in idealized one- and two-particle scattering problems. This simple discussion highlights the connection between symmetry of the wave functions (the Pauli principle) and the 
fluctuation properties. It introduces in a simple manner some of the basic concepts and it will be interesting to compare the results of the one- and two-particle scattering problems with the many-particle problem which we face in mesoscopic conductors.

\section{B. The Pauli principle}

The investigation of the noise properties of a system is interesting because it is fundamentally connected with the statistical properties of the entities which generate the noise. We are concerned with systems which contain a large number of indistinguishable particles. The fact that in quantum mechanics we cannot label different particles implies that the wave function must be invariant, up to a phase, if two particles are exchanged. The invariance of the wave function under exchange of two particles implies that we deal with wavefunctions which are either symmetric or antisymmetric under particle exchange. (In strictly two dimensional systems more exotic possibilities are permitted). These symmetry statements are known as the Pauli principle. Systems with symmetric (antisymmetric) wavefunctions are described by Bose-Einstein (Fermi-Dirac) statistics, respectively.

Prior to the discussion of the noise properties in electrical conductors, which is our central subject, in this subsection we illustrate in a simple manner the fundamental connection between the symmetry of the wave function and the statistical properties of scattering experiments. We deal with open systems similarly to a scattering experiment in which particles are incident on a target at which they are scattered. The simplest case in which the symmetry of the wave function matters is the case of two identical particles. Here we present a discussion of idealized two-particle scattering experiments. We consider the arrangement shown in Fig. 1, which contains two sources 1 and 2 which can emit particles and two detectors 3 and 4 which respond ideally with a signal each time a particle enters a detector. An arrangement similar to that shown in Fig. 1 is used in optical experiments. In this field experiments which invoke one or two incoming particle streams (photons) and two detectors are known as Hanbury Brown - Twiss experiments [7], after the pioneering experiments carried out by these two researchers to investigate the statistical properties of light.

In Fig. 11 the scattering is, much as in an optical table top experiment, provided by a half-silvered mirror (beam-splitter), which permits transmission from the input-channel 1 through the mirror with probability amplitude $s_{41}=t$ to the detector at arm 4 and generates reflected particles with amplitude $s_{31}=r$ into detector 3 . We assume that particles from source 2 are scattered likewise and have probability amplitudes $s_{32}=t$ and $s_{42}=r$. The elements $s_{i j}$, when written as a matrix, form the scattering matrix $s$. The elements of the scattering matrix satisfy $|r|^{2}+|t|^{2}=1$ and $t r^{*}+r t^{*}=0$, stating that the scattering matrix is unitary. A simple example of a scattering matrix often employed to describe scattering at a mirror in optics is $r=-i / \sqrt{2}$ and $t=1 / \sqrt{2}$.

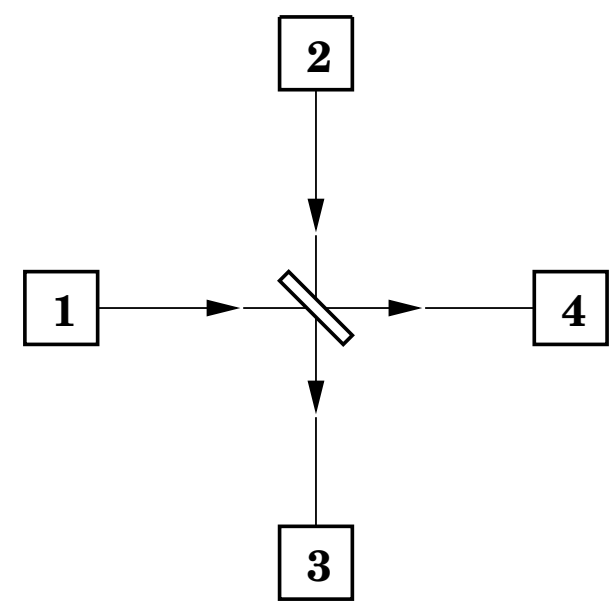

FIG. 1. An arrangement of scattering experiment with two sources (1 and 2) and two detectors (3 and 4).

We are interested in describing various input states emanating from the two sources. To be interesting, these input states contain one or two particles. We could describe these states in terms of Slater determinants, but it is more elegant to employ the second quantization approach. The incident states are described by annihilation operators $\hat{a}_{i}$ or creation operators $\hat{a}_{i}^{\dagger}$ in $\operatorname{arm} i, i=1,2$. The outgoing states are, in turn, described by annihilation operators $\hat{b}_{i}$ and creation operators $\hat{b}_{i}^{\dagger}, i=3,4$. The operators of the input states and output states are not independent but are related by a unitary transformation which is just the scattering matrix,

$$
\left(\begin{array}{l}
\hat{b}_{3} \\
\hat{b}_{4}
\end{array}\right)=s\left(\begin{array}{l}
\hat{a}_{1} \\
\hat{a}_{2}
\end{array}\right) .
$$

Similarly, the creation operators $\hat{a}_{i}^{\dagger}$ and $\hat{b}_{i}^{\dagger}$ are related by the adjoint of the scattering matrix $s^{\dagger}$. Note that the mirror generates quantum mechanical superpositions of the input states. The coefficients of these superpositions are determined by the elements of the scattering matrix.

For bosons, the $\hat{a}_{i}$ obey the commutation relations

$$
\left[\hat{a}_{i}, \hat{a}_{j}^{\dagger}\right]=\delta_{i j}
$$

Since the scattering matrix is unitary, the $\hat{b}_{i}$ obey the same commutation relations. In contrast, for fermions, the $\hat{a}_{i}$ and $\hat{b}_{i}$ obey anti-commutation relations

$$
\left\{\hat{a}_{i}, \hat{a}_{j}^{\dagger}\right\}=\delta_{i j}
$$

The different commutation relations for fermions and bosons assure that multi-particle states reflect the underlying symmetry of the wave function. 
The occupation numbers of the incident and transmitted states are found as $\hat{n}_{i}=\hat{a}_{i}^{\dagger} \hat{a}_{i}$ and $\hat{n}_{i}=\hat{b}_{i}^{\dagger} \hat{b}_{i}$,

$$
\left(\begin{array}{c}
\left\langle\hat{n}_{3}\right\rangle \\
\left\langle\hat{n}_{4}\right\rangle
\end{array}\right)=\left(\begin{array}{cc}
R & T \\
T & R
\end{array}\right)\left(\begin{array}{c}
\left\langle\hat{n}_{1}\right\rangle \\
\left\langle\hat{n}_{2}\right\rangle
\end{array}\right),
$$

where we introduced transmission and reflection probabilities, $T=|t|^{2}$ and $R=|r|^{2}$.

Single, independent particle scattering. Before treating two-particle states it is useful to consider briefly a series of scattering experiments in each of which only one particle is incident on the mirror. Let us suppose that a particle is incident in arm 1 . Since we know that in each scattering experiment there is one incident particle, the average occupation number in the incident arm is thus $\left\langle n_{1}\right\rangle=1$. The fluctuations away from the average occupation number $\Delta n_{1}=n_{1}-\left\langle n_{1}\right\rangle$ vanish identically (not only on the average). In particular, we have $\left\langle\left(\Delta n_{1}\right)^{2}\right\rangle=0$. Particles are transmitted into arm 4 with probability $T$, and thus the mean occupation number in the transmitted beam is $\left\langle n_{4}\right\rangle=T$. Similarly, particles are reflected into arm 3 with probability $R$, and the average occupation number in our series of experiments is thus $\left\langle n_{3}\right\rangle=R$. Consider now the correlation $\left\langle n_{3} n_{4}\right\rangle$ between the occupation numbers in arm 3 and 4 . Since each particle is either reflected or transmitted, it means that in this product one of the occupation numbers is always 1 and one is zero in each experiment. Consequently the correlation of the occupation numbers vanishes, $\left\langle n_{3} n_{4}\right\rangle=0$. Using this result, we obtain for the fluctuations of the occupation numbers $\Delta n_{3}=n_{3}-\left\langle n_{3}\right\rangle$ in the reflected beam and $\Delta n_{4}=n_{4}-\left\langle n_{4}\right\rangle$ in the transmitted beam,

$$
\left\langle\left(\Delta n_{3}\right)^{2}\right\rangle=\left\langle\left(\Delta n_{4}\right)^{2}\right\rangle=-\left\langle\Delta n_{3} \Delta n_{4}\right\rangle=T R .
$$

The fluctuations in the occupation numbers in the transmitted and reflected beams and their correlation are a consequence of the fact that a carrier can finally only be either reflected or transmitted. These fluctuations are known as partition noise. The partition noise vanishes for a completely transparent scatterer $T=1$ and for a completely reflecting scatterer $R=1$ and is maximal for $T=R=1 / 2$. We emphasize that the partition noise is the same, whether we use a fermion or boson in the series of experiments. To detect the sensitivity to the symmetry of the wave function, we need to consider at least two particles.

Two-particle scattering. We now consider two particles incident on the mirror of Fig. 1, focusing on the case that one particle is incident in each arm. We follow here closely a discussion by Loudon [8] and refer the reader to this work for additional information. The empty (vacuum) state of the system (input arms and output arms) is denoted by $|0\rangle$. Consider now an input state which consists of two particles with a definite momentum, one incident in arm 1 and one incident in arm 2. For simplicity, we assume that the momentum of both particles is the same. With the help of the creation operators given above we can generate the input state $|\Psi\rangle=\hat{a}_{1}^{\dagger} \hat{a}_{2}^{\dagger}|0\rangle$. The probability that both particles appear in output arm 3 is $P(2,0)=\left\langle\Psi\left|\hat{n}_{3} \hat{n}_{3}\right| \Psi\right\rangle$, the probability that in each output arm there is one particle is $P(1,1)=\left\langle\Psi\left|\hat{n}_{3} \hat{n}_{4}\right| \Psi\right\rangle$, and the probability that both particles are scattered into arm 4 is $P(0,2)=\left\langle\Psi\left|\hat{n}_{4} \hat{n}_{4}\right| \Psi\right\rangle$. Considering specifically the probability $P(1,1)$, we have to find

$$
P(1,1)=\left\langle\Psi\left|\hat{n}_{3} \hat{n}_{4}\right| \Psi\right\rangle=\left\langle 0\left|\hat{a}_{2} \hat{a}_{1} \hat{b}_{3}^{\dagger} \hat{b}_{3} \hat{b}_{4}^{\dagger} \hat{b}_{4} \hat{a}_{1}^{\dagger} \hat{a}_{2}^{\dagger}\right| 0\right\rangle
$$

First we notice that in the sequence of $\hat{b}$-operators $\hat{b}_{3}$ and $\hat{b}_{4}^{\dagger}$ anti-commute (commute), and we can thus write $\hat{b}_{3}^{\dagger} \hat{b}_{3} \hat{b}_{4}^{\dagger} \hat{b}_{4}$ also in the sequence $\mp \hat{b}_{3}^{\dagger} \hat{b}_{4}^{\dagger} \hat{b}_{3} \hat{b}_{4}$. Then, by inserting a complete set of states with fixed number of particles $|n\rangle\langle n|$ into this product, $\mp \hat{b}_{3}^{\dagger} \hat{b}_{4}^{\dagger}|n\rangle\langle n| \hat{b}_{3} \hat{b}_{4}$, it is only the state with $n=0$ which contributes since to the right of $\langle n|$ we have two creation and two annihilation operators. Thus the probability $P(1,1)$ is given by the absolute square of a probability amplitude

$$
P(1,1)=\left|\left\langle 0\left|\hat{b}_{3} \hat{b}_{4} \hat{a}_{1}^{\dagger} \hat{a}_{2}^{\dagger}\right| 0\right\rangle\right|^{2} .
$$

To complete the evaluation of this probability, we express $\hat{a}_{1}^{\dagger}$ and $\hat{a}_{2}^{\dagger}$ in terms of the output operators $\hat{b}_{3}^{\dagger}$ and $\hat{b}_{4}^{\dagger}$ using the adjoint of Eq. (12). This gives

$$
\hat{a}_{1}^{\dagger} \hat{a}_{2}^{\dagger}=r t \hat{b}_{3}^{\dagger} \hat{b}_{3}^{\dagger}+r^{2} \hat{b}_{3}^{\dagger} \hat{b}_{4}^{\dagger}+t^{2} \hat{b}_{4}^{\dagger} \hat{b}_{3}^{\dagger}+r t \hat{b}_{4}^{\dagger} \hat{b}_{4}^{\dagger} .
$$

Now, using the commutation relations, we pull the annihilation operators to the right until one of them acts on the vacuum and the corresponding term vanishes. A little algebra gives

$$
P(1,1)=(T \pm R)^{2} .
$$

Eq. (20) is a concise statement of the Pauli principle. For bosons $P(1,1)$ depends on the transmission and reflection probability of the scatterer, and vanishes for an ideal mirror $T=R=1 / 2$. The two particles are preferentially scattered into the same output branch. For fermions $P(1,1)$ is independent of the transmission and reflection probability and given by $P(1,1)=1$. Thus fermions are scattered with probability one into the different output branches. It is instructive to compare these result with the one for classical particles, $P(1,1)=T^{2}+R^{2}$. We see thus that the probability to find two bosons (fermions) in two different detectors is suppressed (enhanced) in comparison with the same probability for classical particles.

A similar consideration also gives for the probabilities

$$
P(2,0)=P(0,2)=2 R T
$$

for bosons, whereas for fermions the two probabilities vanish $P(2,0)=P(0,2)=0$. For classical partition of carriers the probability to find the two particles in the same detector is $R T$, which is just one half of the probability for bosons.

The average occupation numbers are $\left\langle n_{3}\right\rangle=\left\langle n_{4}\right\rangle=1$, since we have now two particles in branch 3 with probability $P(2,0)$ and one particle with probability $P(1,1)$. 
Consequently, the correlations of the fluctuations in the occupation numbers $\Delta \hat{n}_{i}=\hat{n}_{i}-\left\langle n_{i}\right\rangle$ are given by

$$
\left\langle\Delta \hat{n}_{3} \Delta \hat{n}_{4}\right\rangle=-4 R T,
$$

for bosons and by $\left\langle\Delta \hat{n}_{3} \Delta \hat{n}_{4}\right\rangle=0$ for fermions. For bosons the correlation is negative due to the enhanced probability that both photons end up in the same output branch. For fermions there are no fluctuations in the occupation number and the correlation function thus vanishes.

Two-particle scattering: Wave packet overlap. The discussion given above implicitly assumes that both "particles" or "waves" arrive simultaneously at the mirror and "see" each other. Clearly, if the two particles arrive at the mirror with a time-delay which is large enough such that there is no overlap, the outcome of the experiments described above is entirely different. If we have only a sequence of individual photons or electrons arriving at the mirror we have for the expectation values of the occupation numbers $\left\langle n_{3}\right\rangle=\left\langle n_{4}\right\rangle=R+T=1$, and the correlation of the occupation number $\left\langle n_{3} n_{4}\right\rangle=0$ vanishes. Consequently, the correlation of the fluctuations of the occupation number is $\left\langle\Delta n_{1} \Delta n_{2}\right\rangle=-1$. Without any special sources at hand it is impossible to time the carriers such that they arrive simultaneously at the mirror, and we should consider all possibilities.

To do this, we must consider the states at the input in more detail. Let us assume that a state in input arm $i$ can be written with the help of plane waves $\Psi_{i}\left(k, x_{i}\right)=\exp \left(-i k x_{i}\right)$ with $x_{i}$ the coordinate along arm $i$ normalized such that it grows as we move away from the arm toward the source. Similarly, let $y_{i}$ be the coordinates along the output arms such that $y_{i}$ vanishes at the mirror and grows as we move away from the splitter. A plane wave $\Psi_{1}\left(k, x_{1}\right)=\exp \left(-i k x_{1}\right)$ incident from arm 1 thus leads to a reflected wave in output arm 3 given by $\Psi_{1}\left(k, y_{3}\right)=r \exp \left(-i k y_{3}\right)$ and to a transmitted wave $\Psi_{1}\left(k, y_{4}\right)=t \exp \left(-i k y_{4}\right)$ in output arm 4 . We call such a state a "scattering state". It can be regarded as the limit of a wave packet with a spatial width that tends towards infinity and an energy width that tends to zero. To built up a particle that is localized in space at a given time we now invoke superpositions of such scattering states. Thus, let the incident particle in arm $i$ be described by $\Psi_{i}\left(x_{i}, t\right)=\int d k \alpha_{i}(k) \exp \left(-i k x_{i}\right) \exp (-i E(k) t / \hbar)$, where $\alpha_{i}(k)$ is a function such that

$$
\int_{0}^{\infty} d k\left|\alpha_{i}(k)\right|^{2}=1
$$

and $E(k)$ is the energy of the carriers as a function of the wave vector $k$. In second quantization the incident states are written with the help of the operators

$$
\begin{aligned}
\hat{A}_{i}^{\dagger}\left(x_{i}, t\right) & =\int_{0}^{\infty} d k_{i} \alpha_{i}\left(k_{i}\right) \Psi_{i}\left(k, x_{i}\right) \hat{a}_{i}^{\dagger}\left(k_{i}\right) \\
& \times \exp \left(-i E\left(k_{i}\right) t / \hbar\right),
\end{aligned}
$$

and the initial state of our two-particle scattering experiment is thus $\hat{A}_{1}^{\dagger}\left(x_{1}, t\right) \hat{A}_{2}^{\dagger}\left(x_{2}, t\right)|0\rangle$. We are again interested

in determining the probabilities that two particles appear in an output branch or that one particle appears in each output branch, $P(2,0)=\int_{0}^{\infty} d k_{3} d k_{4}\left\langle\hat{n}_{3}\left(k_{3}\right) \hat{n}_{3}\left(k_{4}\right)\right\rangle$, $P(1,1)=\int_{0}^{\infty} d k_{3} d k_{4}\left\langle\hat{n}_{3}\left(k_{3}\right) \hat{n}_{4}\left(k_{4}\right)\right\rangle$, and $P(0,2)=$ $\int_{0}^{\infty} d k_{3} d k_{4}\left\langle\hat{n}_{4}\left(k_{3}\right) \hat{n}_{4}\left(k_{4}\right)\right\rangle$. Let us again consider $P(1,1)$. Its evaluation proceeds in much the same way as in the case of pure scattering states. We re-write $P(1,1)$ in terms of the absolute square of an amplitude,

$$
P(1,1)=\int_{0}^{\infty} d k_{3} d k_{4}\left|\left\langle 0\left|\hat{b}_{3}\left(k_{3}\right) \hat{b}_{4}\left(k_{4}\right)\right| \Psi\right\rangle\right|^{2} .
$$

We then write the $\hat{a}$ operators in the $\hat{A}$ in terms of the output operators $\hat{b}$. Instead of Eq. (20), we obtain

$$
P(1,1)=T^{2}+R^{2} \pm 2 T R|J|^{2},
$$

where

$$
J=\int_{0}^{\infty} d k \alpha_{1}^{*}(k) \alpha_{2}(k) \exp \left(i k\left(x_{1}-x_{2}\right)\right)
$$

is the overlap integral of the two particles. For the case of complete overlap $|J|=1$ we obtain Eq. (20). For the case that we have no overlap we obtain the classical result $P(1,1)=T^{2}+R^{2}$ which is independent of whether a boson or fermion is incident on the scatterer. In the general case, the overlap depends on the form of the wave packet. If two Gaussian wave packets of spatial width $\delta$ and central velocity $v$ are timed to arrive at time $\tau_{1}$ and $\tau_{2}$ at the scatterer, the overlap integral is

$$
|J|^{2}=\exp \left[-v^{2}\left(\tau_{1}-\tau_{2}\right)^{2} / 2 \delta^{2}\right]
$$

A significant overlap occurs only during the time $\delta / v$. For wave packets separated in time by more than this time interval the Pauli principle is not effective.

Complete overlap occurs in two simple cases. We can assume that the two wave packets are identical and are timed to arrive exactly at the same instant at the scatterer. Another case, in which we have complete overlap, is in the basis of scattering states. In this case $\left|\alpha_{i}(k)\right|^{2}=\delta\left(k-k_{i}\right)$ for a scattering state with wave vector $k_{i}$ and consequently for the two particles with $k_{i}$ and $k_{j}$ we have $J=\delta_{i j}$. The first option of timed wave packets seems artificial for the thermal sources which we want to describe. Thus in the following we will work with scattering states.

\begin{tabular}{llll}
\hline \hline Probability & Classical & Bosons & Fermions \\
\hline$P(2,0)$ & $R T$ & $R T\left(1+|J|^{2}\right)$ & $R T\left(1-|J|^{2}\right)$ \\
$P(1,1)$ & $R^{2}+T^{2}$ & $R^{2}+T^{2}-2 R T|J|^{2}$ & $R^{2}+T^{2}+2 R T|J|^{2}$ \\
$P(0,2)$ & $R T$ & $R T\left(1+|J|^{2}\right)$ & $R T\left(1-|J|^{2}\right)$ \\
\hline \hline
\end{tabular}

TABLE II. Output probabilities for one particle incident in each input arm (From Ref. [8]). 
The probabilities $P(2,0), P(1,1)$ and $P(0,2)$ for these scattering experiments are shown in Table II. The considerations which lead to these results now should be extended to take the polarization of photons or spin of electrons into account.

Two-particle scattering: Spin. Consider the case of fermions and let us investigate a sequence of experiments in each of which we have an equal probability of having electrons with spin up or down in an incident state. Assuming that the scattering matrix is independent of the spin state of the electrons, the results discussed above describe the two cases when both spins point in the same direction (to be denoted as $P(1 \uparrow, 1 \uparrow$ ) and $P(1 \downarrow, 1 \downarrow))$. Thus what remains is to consider the case in which one incident particle has spin up and one incident particle has spin down. If the detection is also spin sensitive, the probability which we determine is $P(1 \uparrow, 1 \downarrow)$. But in such an experiment we can tell which of the two particles went which way at the scatterer and there is thus no interference. The outcome is classical: $P(1 \uparrow, 1 \downarrow)=R^{2}$ for an initial state with a spin up in arm 1 and a spin down in arm 2. For the same state we have $P(1 \downarrow, 1 \uparrow)=T^{2}$.

Now let us assume that there is no way of detecting the spin state of the outgoing particles. For a given initial state we have $P(1,1)=\sum_{\sigma, \sigma^{\prime}} P\left(1 \sigma, 1 \sigma^{\prime}\right)$ where $\sigma$ and $\sigma^{\prime}$ are spin variables. If we consider all possible incident states with equal probability, we find $P(1,1)=T^{2}+R^{2}+$ $T R|J|^{2}$ i. e. a result with an interference contribution which is only half as large as given in Eq. (26). For further discussion, see Appendix B.

The scattering experiments considered above assume that we can produce one or two particle states either in a single mode or by exciting many modes. Below we will show that thermal sources, the electron reservoirs which are of the main interest here, cannot be described in this way.

\section{The scattering approach}

The idea of the scattering approach (also referred to as Landauer approach) is to relate transport properties of the system (in particular, current fluctuations) to its scattering properties, which are assumed to be known from a quantum-mechanical calculation. In its traditional form the method applies to non-interacting systems in the stationary regime 2 . The system may be either

\footnotetext{
${ }^{2}$ To avoid a possible misunderstanding, we stress that the long range Coulomb interaction needs to be taken into account when one tries to apply the scattering approach for the description of systems in time-dependent external fields, or finite-frequency fluctuation spectra in stationary fields. On the other hand, for the description of zero-frequency fluctua-
}

at equilibrium or in a non-equilibrium state; this information is introduced through the distribution functions of the contacts of the sample. To be clear, we consider first a two-probe geometry and particles obeying Fermi statistics (having in mind electrons in mesoscopic systems). Eventually, the generalization to many probes and Bose statistics is given; extensions to interacting problems are discussed at the end of this Section. In the derivation we essentially follow Ref. [9].

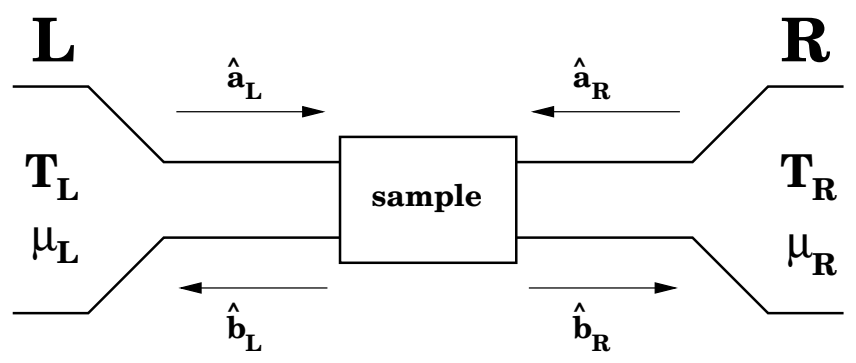

FIG. 2. Example of two-terminal scattering problem for the case of one transverse channel.

Two-terminal case; current operator. We consider a mesoscopic sample connected to two reservoirs (terminals, probes), to be referred to as "left" (L) and "right" (R). It is assumed that the reservoirs are so large that they can be characterized by a temperature $T_{L, R}$ and a chemical potential $\mu_{L, R}$; the distribution functions of electrons in the reservoirs, defined via these parameters, are then Fermi distribution functions

$$
f_{\alpha}(E)=\left[\exp \left[\left(E-\mu_{\alpha}\right) / k_{B} T_{\alpha}\right]+1\right]^{-1}, \quad \alpha=L, R
$$

(see Fig. 2). We must note at this stage, that, although there are no inelastic processes in the sample, a strict equilibrium state in the reservoirs can be established only via inelastic processes. However, we consider the reservoirs (the leads) to be wide compared to the typical crosssection of the mesoscopic conductor. Consequently, as far as the reservoirs are concerned, the mesoscopic conductor represents only a small perturbation, and describing their local properties in terms of an equilibrium state is thus justified. We emphasize here, that even though the dynamics of the scattering problem is described in terms of a Hamiltonian, the problem which we consider is irreversible. Irreversibility is introduced in the discussion, since the processes of a carrier leaving the mesoscopic conductor and entering the mesoscopic conductor are unrelated, uncorrelated events. The reservoirs act as sources of carriers determined by the Fermi distribution

tion spectra in stationary fields, a consistent theory can be given without including Coulomb effects, even though the fluctuations themselves are, of course, time-dependent and random. 
but also act as perfect sinks of carriers irrespective of the energy of the carrier that is leaving the conductor.

Far from the sample, we can, without loss of generality, assume that transverse (across the leads) and longitudinal (along the leads) motion of electrons are separable. In the longitudinal (from left to right) direction the system is open, and is characterized by the continuous wave vector $k_{l}$. It is advantageous to separate incoming (to the sample) and outgoing states, and to introduce the longitudinal energy $E_{l}=\hbar^{2} k_{l}^{2} / 2 m$ as a quantum number. Transverse motion is quantized and described by the discrete index $n$ (corresponding to transverse energies $E_{L, R ; n}$, which can be different for the left and right leads). These states are in the following referred to as transverse (quantum) channels. We write thus $E=E_{n}+E_{l}$. Since $E_{l}$ needs to be positive, for a given total energy $E$ only a finite number of channels exists. The number of incoming channels is denoted $N_{L, R}(E)$ in the left and right lead, respectively.

We now introduce creation and annihilation operators of electrons in the scattering states. In principle, we could have used the operators which refer to particles in the states described by the quantum numbers $n, k_{l}$. However, the scattering matrix which we introduce below, relates current amplitudes and not wave function amplitudes. Thus we introduce operators $\hat{a}_{L n}^{\dagger}(E)$ and $\hat{a}_{L n}(E)$ which create and annihilate electrons with total energy $E$ in the transverse channel $n$ in the left lead, which are incident upon the sample. In the same way, the creation $\hat{b}_{L n}^{\dagger}(E)$ and annihilation $\hat{b}_{L n}(E)$ operators describe electrons in the outgoing states. They obey anticommutation relations

$$
\begin{gathered}
\hat{a}_{L n}^{\dagger}(E) \hat{a}_{L n^{\prime}}\left(E^{\prime}\right)+\hat{a}_{L n^{\prime}}\left(E^{\prime}\right) \hat{a}_{L n}^{\dagger}(E)=\delta_{n n^{\prime}} \delta\left(E-E^{\prime}\right), \\
\hat{a}_{L n}(E) \hat{a}_{L n^{\prime}}\left(E^{\prime}\right)+\hat{a}_{L n^{\prime}}\left(E^{\prime}\right) \hat{a}_{L n}(E)=0, \\
\hat{a}_{L n}^{\dagger}(E) \hat{a}_{L n^{\prime}}^{\dagger}\left(E^{\prime}\right)+\hat{a}_{L n^{\prime}}^{\dagger}\left(E^{\prime}\right) \hat{a}_{L n}^{\dagger}(E)=0 .
\end{gathered}
$$

Similarly, we introduce creation and annihilation operators $\hat{a}_{R n}^{\dagger}(E)$ and $\hat{a}_{R n}(E)$ in incoming states and $\hat{b}_{R n}^{\dagger}(E)$ and $\hat{b}_{R n}(E)$ in outgoing states in the right lead (Fig. 2).

The operators $\hat{a}$ and $\hat{b}$ are related via the scattering matrix $s$,

$$
\left(\begin{array}{c}
\hat{b}_{L 1} \\
\cdots \\
\hat{b}_{L N_{L}} \\
\hat{b}_{R 1} \\
\cdots \\
\hat{b}_{R N_{R}}
\end{array}\right)=s\left(\begin{array}{c}
\hat{a}_{L 1} \\
\cdots \\
\hat{a}_{L N_{L}} \\
\hat{a}_{R 1} \\
\cdots \\
\hat{a}_{R N_{R}}
\end{array}\right) .
$$

The creation operators $\hat{a}^{\dagger}$ and $\hat{b}^{\dagger}$ obey the same relation with the hermitian conjugated matrix $s^{\dagger}$.

The matrix $s$ has dimensions $\left(N_{L}+N_{R}\right) \times\left(N_{L}+N_{R}\right)$. Its size, as well as the matrix elements, depends on the total energy $E$. It has the block structure

$$
s=\left(\begin{array}{cc}
r & t^{\prime} \\
t & r^{\prime}
\end{array}\right)
$$

Here the square diagonal blocks $r$ (size $N_{L} \times N_{L}$ ) and $r^{\prime}$ (size $N_{R} \times N_{R}$ ) describe electron reflection back to the left and right reservoirs, respectively. The off-diagonal, rectangular blocks $t\left(\right.$ size $\left.N_{R} \times N_{L}\right)$ and $t^{\prime}\left(\right.$ size $\left.N_{L} \times N_{R}\right)$ are responsible for the electron transmission through the sample. The flux conservation in the scattering process implies that the matrix $s$ is quite generally unitary. In the presence of time-reversal symmetry the scattering matrix is also symmetric.

The current operator in the left lead (far from the sample) is expressed in a standard way,

$$
\begin{aligned}
\hat{I}_{L}(z, t) & =\frac{\hbar e}{2 i m} \int d \boldsymbol{r}_{\perp}\left[\hat{\Psi}_{L}^{\dagger}(\boldsymbol{r}, t) \frac{\partial}{\partial z} \hat{\Psi}_{L}(\boldsymbol{r}, t)\right. \\
& \left.-\left(\frac{\partial}{\partial z} \hat{\Psi}_{L}^{\dagger}(\boldsymbol{r}, t)\right) \hat{\Psi}_{L}(\boldsymbol{r}, t)\right],
\end{aligned}
$$

where the field operators $\hat{\Psi}$ and $\hat{\Psi}^{\dagger}$ are defined as

$$
\begin{aligned}
\hat{\Psi}_{L}(\boldsymbol{r}, t) & =\int d E e^{-i E t / \hbar} \sum_{n=1}^{N_{L}(E)} \frac{\chi_{L n}\left(\boldsymbol{r}_{\perp}\right)}{\left(2 \pi \hbar v_{L n}(E)\right)^{1 / 2}} \\
& \times\left[\hat{a}_{L n} e^{i k_{L n} z}+\hat{b}_{L n} e^{-i k_{L n} z}\right]
\end{aligned}
$$

and

$$
\begin{aligned}
\hat{\Psi}_{L}^{\dagger}(\boldsymbol{r}, t) & =\int d E e^{i E t / \hbar} \sum_{n=1}^{N_{L}(E)} \frac{\chi_{L n}^{*}\left(\boldsymbol{r}_{\perp}\right)}{\left(2 \pi \hbar v_{L n}(E)\right)^{1 / 2}} \\
& \times\left[\hat{a}_{L n}^{\dagger} e^{-i k_{L n} z}+\hat{b}_{L n}^{\dagger} e^{i k_{L n} z}\right] .
\end{aligned}
$$

Here $\boldsymbol{r}_{\perp}$ is the transverse coordinate(s) and $z$ is the coordinate along the leads (measured from left to right); $\chi_{n}^{L}$ are the transverse wave functions, and we have introduced the wave vector, $k_{L n}=\hbar^{-1}\left[2 m\left(E-E_{L n}\right)\right]^{1 / 2}$ (the summation only includes channels with real $k_{L n}$ ), and the velocity of carriers $v_{n}(E)=\hbar k_{L n} / m$ in the $n$-th transverse channel.

After some algebra, the expression for the current can be cast into the form

$$
\begin{aligned}
\hat{I}_{L}(z, t) & =\frac{e}{4 \pi \hbar} \sum_{n} \int d E d E^{\prime} e^{i\left(E-E^{\prime}\right) t / \hbar} \frac{1}{\sqrt{v_{L n}(E) v_{L n}\left(E^{\prime}\right)}} \\
& \times\left\{\left[v_{L n}(E)+v_{L n}\left(E^{\prime}\right)\right]\right. \\
& \times\left[\exp \left[i\left(k_{L n}\left(E^{\prime}\right)-k_{L n}(E)\right) z\right] \hat{a}_{L n}^{\dagger}(E) \hat{a}_{L n}\left(E^{\prime}\right)\right. \\
& \left.-\exp \left[i\left(k_{L n}(E)-k_{L n}\left(E^{\prime}\right)\right) z\right] \hat{b}_{L n}^{\dagger}(E) \hat{b}_{L n}\left(E^{\prime}\right)\right] \\
& +\left[v_{L n}(E)-v_{L n}\left(E^{\prime}\right)\right] \\
& \times\left[\exp \left[-i\left(k_{L n}(E)+k_{L n}\left(E^{\prime}\right)\right) z\right] \hat{a}_{L n}^{\dagger}(E) \hat{b}_{L n}\left(E^{\prime}\right)\right. \\
& \left.\left.-\exp \left[i\left(k_{L n}(E)+k_{L n}\left(E^{\prime}\right)\right) z\right] \hat{b}_{L n}^{\dagger}(E) \hat{a}_{L n}\left(E^{\prime}\right)\right]\right\} .
\end{aligned}
$$


This expression is cumbersome, and, in addition, depends explicitly on the coordinate $z$. However, it can be considerably simplified. The key point is that for all observable quantities (average current, noise, or higher moments of the current distribution) the energies $E$ and $E^{\prime}$ in Eq. (31) either coincide, or are close to each other. On the other hand, the velocities $v_{n}(E)$ vary with energy quite slowly, typically on the scale of the Fermi energy. Therefore, one can neglect their energy dependence, and reduce the expression (31) to a much simpler form?

$$
\begin{aligned}
\hat{I}_{L}(t) & =\frac{e}{2 \pi \hbar} \sum_{n} \int d E d E^{\prime} e^{i\left(E-E^{\prime}\right) t / \hbar} \\
& \times\left[\hat{a}_{L n}^{\dagger}(E) \hat{a}_{L n}\left(E^{\prime}\right)-\hat{b}_{L n}^{\dagger}(E) \hat{b}_{L n}\left(E^{\prime}\right)\right] .
\end{aligned}
$$

Note that $\hat{n}_{L n}^{+}(E)=\hat{a}_{L n}^{\dagger}(E) \hat{a}_{L n}(E)$ is the operator of the occupation number of the incident carriers in lead $L$ in channel $n$. Similarly, $\hat{n}_{L n}^{-}(E)=\hat{b}_{L n}^{\dagger}(E) \hat{b}_{L n}(E)$ is the operator of the occupation number of the out-going carriers in lead $L$ in channel $n$. Setting $E^{\prime}=E+\hbar \omega$ and carrying out the integral over $\omega$ gives

$$
\hat{I}_{L}(t)=\frac{e}{2 \pi \hbar} \sum_{n} \int d E\left[\hat{n}_{L n}^{+}(E, t)-\hat{n}_{L n}^{-}(E, t)\right] .
$$

Here $\hat{n}_{L n}^{ \pm}(E, t)$ are the time-dependent occupation numbers for the left and right moving carriers at energy $E$. Thus Eq. (33) states that the current at time $t$ is simply determined by the difference in occupation number between the left and right movers in each channel. We made use of this intuitively appealing result already in the introduction. Using Eq. (29) we can express the current in terms of the $\hat{a}$ and $\hat{a}^{\dagger}$ operators alone,

$$
\begin{aligned}
\hat{I}_{L}(t) & =\frac{e}{2 \pi \hbar} \sum_{\alpha \beta} \sum_{m n} \int d E d E^{\prime} e^{i\left(E-E^{\prime}\right) t / \hbar} \\
& \times \hat{a}_{\alpha m}^{\dagger}(E) A_{\alpha \beta}^{m n}\left(L ; E, E^{\prime}\right) \hat{a}_{\beta n}\left(E^{\prime}\right) .
\end{aligned}
$$

Here the indices $\alpha$ and $\beta$ label the reservoirs and may assume values $L$ or $R$. The matrix $A$ is defined as

$$
A_{\alpha \beta}^{m n}\left(L ; E, E^{\prime}\right)=\delta_{m n} \delta_{\alpha L} \delta_{\beta L}-\sum_{k} s_{L \alpha ; m k}^{\dagger}(E) s_{L \beta ; k n}\left(E^{\prime}\right),
$$

and $s_{L \alpha ; m k}(E)$ is the element of the scattering matrix relating $\hat{b}_{L m}(E)$ to $\hat{a}_{\alpha k}(E)$. Note that Eq. (34) is independent of the coordinate $z$ along the lead.

Average current. Before we proceed in the next subsection with the calculation of current-current correlations, it is instructive to derive the average current

${ }^{3}$ A discussion of the limitations of Eq. (32) is given in Ref. [10]. from Eq. (34). For a system at thermal equilibrium the quantum statistical average of the product of an electron creation operator and annihilation operator of a Fermi gas is

$$
\left\langle\hat{a}_{\alpha m}^{\dagger}(E) \hat{a}_{\beta n}\left(E^{\prime}\right)\right\rangle=\delta_{\alpha \beta} \delta_{m n} \delta\left(E-E^{\prime}\right) f_{\alpha}(E) .
$$

Using Eq. (34) and Eq. (36) and taking into account the unitarity of the scattering matrix $s$, we obtain

$$
\left\langle I_{L}\right\rangle=\frac{e}{2 \pi \hbar} \int d E \operatorname{Tr}\left[t^{\dagger}(E) t(E)\right]\left[f_{L}(E)-f_{R}(E)\right] .
$$

Here the matrix $t$ is the off-diagonal block of the scattering matrix (30), $t_{m n}=s_{R L ; m n}$. In the zero-temperature limit and for a small applied voltage Eq. (37) gives a conductance

$$
G=\frac{e^{2}}{2 \pi \hbar} \operatorname{Tr}\left[t^{\dagger}\left(E_{F}\right) t\left(E_{F}\right)\right] .
$$

Eq. (38) establishes the relation between the scattering matrix evaluated at the Fermi energy and the conductance. It is a basis invariant expression. The matrix $t^{\dagger} t$ can be diagonalized; it has a real set of eigenvalues (transmission probabilities) $T_{n}(E)$ (not to be confused with temperature), each of them assumes a value between zero and one. In the basis of eigen channels we have instead of Eq. (37)

$$
\left\langle I_{L}\right\rangle=\frac{e}{2 \pi \hbar} \sum_{n} \int d E T_{n}(E)\left[f_{L}(E)-f_{R}(E)\right] .
$$

and thus for the conductance

$$
G=\frac{e^{2}}{2 \pi \hbar} \sum_{n} T_{n},
$$

Eq. (40) is known as a multi-channel generalization of the Landauer formula. Still another version of this result expresses the conductance in terms of the transmission probabilities $T_{R L, m n}=\left|s_{R L, m n}\right|^{2}$ for carriers incident in channel $n$ in the left lead $L$ and transmitted into channel $m$ in the right lead $R$. In this basis the Hamiltonians of the left and right lead (the reservoirs) are diagonal and the conductance is given by

$$
G=\frac{e^{2}}{2 \pi \hbar} \sum_{m n} T_{m n}
$$

We refer to this basis as the natural basis. We remark already here that, independently of the choice of basis, the conductance can be expressed in terms of transmission probabilities only. This is not case for the shot noise to be discussed subsequently. Thus the scattering matrix rather then transmission probabilities represents the fundamental object governing the kinetics of carriers. 
Multi-terminal case. We consider now a sample connected by ideal leads to a number of reservoirs labeled by an index $\alpha$, with the Fermi distribution functions $f_{\alpha}(E)$. At a given energy $E$ the lead $\alpha$ supports $N_{\alpha}(E)$ transverse channels. We introduce, as before, creation and annihilation operators of electrons in an incoming $\hat{a}_{\alpha n}^{\dagger}$, $\hat{a}_{\alpha n}$ and outgoing $\hat{b}_{\alpha n}^{\dagger}, \hat{b}_{\alpha n}$ state of lead $\alpha$ in the transverse channel $n$. These operators are again related via the scattering matrix. We write down this relation, similar to Eq. (29), in components,

$$
\hat{b}_{\alpha m}(E)=\sum_{\beta n} s_{\alpha \beta ; m n}(E) \hat{a}_{\beta n}(E) .
$$

The matrix $s$ is again unitary, and, in the presence of time-reversal symmetry, symmetric.

Proceeding similarly to the derivation presented above, we obtain the multi-terminal generalization of Eq. (34) for the current through the lead $\alpha$,

$$
\begin{aligned}
\hat{I}_{\alpha}(t) & =\frac{e}{2 \pi \hbar} \sum_{\beta \gamma} \sum_{m n} \int d E d E^{\prime} e^{i\left(E-E^{\prime}\right) t / \hbar} \\
& \times \hat{a}_{\beta m}^{\dagger}(E) A_{\beta \gamma}^{m n}\left(\alpha ; E, E^{\prime}\right) \hat{a}_{\gamma n}\left(E^{\prime}\right),
\end{aligned}
$$

with the notation

$$
A_{\beta \gamma}^{m n}\left(\alpha ; E, E^{\prime}\right)=\delta_{m n} \delta_{\alpha \beta} \delta_{\alpha \gamma}-\sum_{k} s_{\alpha \beta ; m k}^{\dagger}(E) s_{\alpha \gamma ; k n}\left(E^{\prime}\right) .
$$

The signs of currents are chosen to be positive for incoming electrons.

Imagine that a voltage $V_{\beta}$ is applied to the reservoir $\beta$, that is, the electro-chemical potential is $\mu_{\beta}=\mu+e V_{\beta}$, where $\mu$ can be taken to be the equilibrium chemical potential. From Eq. (43) we find the average current,

$$
\begin{aligned}
\left\langle I_{\alpha}\right\rangle & =\frac{e^{2}}{2 \pi \hbar} \sum_{\beta} V_{\beta} \int d E\left(-\frac{\partial f}{\partial E}\right) \\
& \times\left[N_{\alpha} \delta_{\alpha \beta}-\operatorname{Tr}\left(s_{\alpha \beta}^{\dagger} s_{\alpha \beta}\right)\right],
\end{aligned}
$$

where the trace is taken over channel indices in lead $\alpha$. As usual, we define the conductance matrix $G_{\alpha \beta}$ via $G_{\alpha \beta}=$ $d\left\langle I_{\alpha}\right\rangle /\left.d V_{\beta}\right|_{V_{\beta}=0}$. In the linear regime this gives

$$
\left\langle I_{\alpha}\right\rangle=\sum_{\beta} G_{\alpha \beta} V_{\beta}
$$

with

$$
G_{\alpha \beta}=\frac{e^{2}}{2 \pi \hbar} \int d E\left(-\frac{\partial f}{\partial E}\right)\left[N_{\alpha} \delta_{\alpha \beta}-\operatorname{Tr}\left(s_{\alpha \beta}^{\dagger} s_{\alpha \beta}\right)\right] .
$$

The scattering matrix is evaluated at the Fermi energy. Eq. (47) has been successfully applied to a wide range of problems from ballistic transport to the quantum Hall effect.

Current conservation, gauge invariance, and reciprocity. Any reasonable theory of electron transport must be current-conserving and gauge invariant. Current conservation means that the sum of currents entering the sample from all terminals is equal to zero at each instant of time. For the multi-terminal geometry discussed here this means $\sum_{\alpha} I_{\alpha}=0$. The current is taken to be positive if it flows from the reservoir towards the mesoscopic structure. For the average current in the two-terminal geometry, we have $I_{L}+I_{R}=0$. We emphasize that current conservation must hold not only on the average but at each instant of time. In particular, current conservation must also hold for the fluctuation spectra which we discuss subsequently. In general, for time dependent currents, we have to consider not only contacts which permit carrier exchange with the conductor, but also other nearby metallic structures, for instance gates, against which the conductor can be polarized. The requirement that the results are gauge invariant means in this context, that no current arises if voltages at all reservoirs are simultaneously shifted by the same value (and no temperature gradient is applied). For the average currents (see Eqs. (39), (47)) both properties are a direct consequence of the unitarity of the scattering matrix.

For the conductance matrix $G_{\alpha \beta}$ current conservation and gauge invariance require that the elements of this matrix in each row and in each column add up to zero,

$$
\sum_{\alpha} G_{\alpha \beta}=\sum_{\beta} G_{\alpha \beta}=0
$$

Note that for the two terminal case this implies $G \equiv$ $G_{L L}=G_{R R}=-G_{L R}=-G_{R L}$. In the two terminal case, it is thus sufficient to evaluate one conductance to determine the conductance matrix. In multi-probe samples the number of elements of the one has to determine to find the conductance matrix is given by the constraints (48) and by the fact that the conductance matrix is a susceptibility and obeys the Onsager-Casimir symmetries

$$
G_{\alpha \beta}(B)=G_{\beta \alpha}(-B) .
$$

In the scattering approach the Onsager-Casimir symmetries are again a direct consequence of the reciprocity symmetry of the scattering matrix under field reversal.

In the stationary case, the current conservation and the gauge invariance of the results are a direct consequence of the unitarity of the scattering matrix. In general, for non-linear and non-stationary problems, current conservation and gauge invariance are not automatically fulfilled. Indeed, in ac-transport a direct calculation of average particle currents does not yield a current conserving theory. Only the introduction of displacement currents, determined by the long range Coulomb interaction, leads to a theory which satisfies these basic requirements. We will discuss these issues for noise problems in Section [III. 


\section{General expressions for noise}

We are concerned with fluctuations of the current away from their average value. We thus introduce the operators $\Delta \hat{I}_{\alpha}(t) \equiv \hat{I}_{\alpha}(t)-\left\langle I_{\alpha}\right\rangle$. We define the correlation function $S_{\alpha \beta}\left(t-t^{\prime}\right)$ of the current in contact $\alpha$ and the current in contact $\beta$ as

$$
S_{\alpha \beta}\left(t-t^{\prime}\right) \equiv \frac{1}{2}\left\langle\Delta \hat{I}_{\alpha}(t) \Delta \hat{I}_{\beta}\left(t^{\prime}\right)+\Delta \hat{I}_{\beta}\left(t^{\prime}\right) \Delta \hat{I}_{\alpha}(t)\right\rangle .
$$

Note that in the absence of time-dependent external fields the correlation function must be function of only $t-t^{\prime}$. Its Fourier transform,

$2 \pi \delta\left(\omega+\omega^{\prime}\right) S_{\alpha \beta}(\omega) \equiv\left\langle\Delta \hat{I}_{\alpha}(\omega) \Delta \hat{I}_{\beta}\left(\omega^{\prime}\right)+\Delta \hat{I}_{\beta}\left(\omega^{\prime}\right) \Delta \hat{I}_{\alpha}(\omega)\right\rangle$, is sometimes referred to as noise power.

To find the noise power we need the quantum statistical expectation value of products of four operators $\hat{a}$. For a Fermi gas (or a Bose gas) at equilibrium this expectation value is

$$
\begin{aligned}
& \left\langle\hat{a}_{\alpha k}^{\dagger}\left(E_{1}\right) \hat{a}_{\beta l}\left(E_{2}\right) \hat{a}_{\gamma m}^{\dagger}\left(E_{3}\right) \hat{a}_{\delta n}\left(E_{4}\right)\right\rangle \\
- & \left\langle\hat{a}_{\alpha k}^{\dagger}\left(E_{1}\right) \hat{a}_{\beta l}\left(E_{2}\right)\right\rangle\left\langle\hat{a}_{\gamma m}^{\dagger}\left(E_{3}\right) \hat{a}_{\delta n}\left(E_{4}\right)\right\rangle \\
= & \delta_{\alpha \delta} \delta_{\beta \gamma} \delta_{k n} \delta_{m l} \delta\left(E_{1}-E_{4}\right) \delta\left(E_{2}-E_{3}\right) \\
\times & f_{\alpha}\left(E_{1}\right)\left[1 \mp f_{\beta}\left(E_{2}\right)\right] .
\end{aligned}
$$

(The upper sign corresponds to Fermi statistics, and the lower sign corresponds to Bose statistics. This convention will be maintained whenever we compare systems with differing statistics. It is also understood that for Fermi statistics $f_{\alpha}(E)$ is a Fermi distribution and for Bose statistics $f_{\alpha}(E)$ is a Bose distribution function). Making use of Eq. 43) and of the expectation value (50), we obtain the expression for the noise power [9],

$$
\begin{aligned}
S_{\alpha \beta}(\omega) & =\frac{e^{2}}{2 \pi \hbar} \sum_{\gamma \delta} \sum_{m n} \int d E A_{\gamma \delta}^{m n}(\alpha ; E, E+\hbar \omega) \\
& \times A_{\delta \gamma}^{n m}(\beta ; E+\hbar \omega, E) \\
& \times\left\{f_{\gamma}(E)\left[1 \mp f_{\delta}(E+\hbar \omega)\right]\right. \\
& \left.+\left[1 \mp f_{\gamma}(E)\right] f_{\delta}(E+\hbar \omega)\right\}
\end{aligned}
$$

\footnotetext{
${ }^{4}$ Note that several definitions, differing by numerical factors, can be found in the literature. The one we use corresponds to the general definition of time-dependent fluctuations found in Ref. [11]. We define the Fourier transform with the coefficient 2 in front of it, then our normalization yields the equilibrium (Nyquist-Johnson) noise $S=4 k_{B} T G$ and is in accordance with Ref. [1], see below. The standard definition of Fourier transform would yield the Nyquist-Johnson noise $S=2 k_{B} T G$. Ref. 9] defines the spectral function which is multiplied by the width of the frequency interval where noise is measured.
}

Note that with respect to frequency, it has the symmetry properties $S_{\alpha \beta}(\omega)=S_{\beta \alpha}(-\omega)$. For arbitrary frequencies and an arbitrary $s$-matrix Eq. (51) is neither current conserving nor gauge invariant and additional considerations are needed to obtain a physically meaningful result.

In the reminder of this Section, we will only be interested in the zero-frequency noise. For the noise power at $\omega=0$ we obtain [9]

$$
\begin{aligned}
S_{\alpha \beta} \equiv S_{\alpha \beta}(0) & =\frac{e^{2}}{2 \pi \hbar} \sum_{\gamma \delta} \sum_{m n} \int d E A_{\gamma \delta}^{m n}(\alpha ; E, E) \\
& \times A_{\delta \gamma}^{n m}(\beta ; E, E) \\
& \times\left\{f_{\gamma}(E)\left[1 \mp f_{\delta}(E)\right]+\left[1 \mp f_{\gamma}(E)\right] f_{\delta}(E)\right\} .
\end{aligned}
$$

Eqs. (52) are current conserving and gauge invariant. Eq. (52) can now be used to predict the low frequency noise properties of arbitrary multi-channel and multiprobe, phase-coherent conductors. We first elucidate the general properties of this result, and later on analyze it for various physical situations.

Equilibrium noise. If the system is in thermal equilibrium at temperature $T$, the distribution functions in all reservoirs coincide and are equal to $f(E)$. Using the property $f(1 \mp f)=-k_{B} T \partial f / \partial E$ and employing the unitarity of the scattering matrix, which enables us to write

$$
\sum_{\gamma \delta} \operatorname{Tr}\left(s_{\alpha \gamma}^{\dagger} s_{\alpha \delta} s_{\beta \delta}^{\dagger} s_{\beta \gamma}\right)=\delta_{\alpha \beta} N_{\alpha}
$$

(where as before the trace is taken over transverse channel indices, and $N_{\alpha}$ is the number of channels in the lead $\alpha)$, we find

$$
\begin{aligned}
S_{\alpha \beta} & =\frac{e^{2} k_{B} T}{\pi \hbar} \int d E\left(-\frac{\partial f}{\partial E}\right) \\
& \times\left[2 N_{\alpha} \delta_{\alpha \beta}-\operatorname{Tr}\left(s_{\alpha \beta}^{\dagger} s_{\alpha \beta}+s_{\beta \alpha}^{\dagger} s_{\beta \alpha}\right)\right] .
\end{aligned}
$$

This is the equilibrium, or Nyquist-Johnson noise. In the approach discussed here it is a consequence of the the thermal fluctuations of occupation numbers in the reservoirs. Comparing Eqs. (45) and (53), we see that

$$
S_{\alpha \beta}=2 k_{B} T\left(G_{\alpha \beta}+G_{\beta \alpha}\right)
$$

This is the manifestation of the fluctuation-dissipation theorem: equilibrium fluctuations are proportional to the corresponding generalized susceptibility, in this case to the conductance. For the time-reversal case (no magnetic field) the conductance matrix is symmetric, and Eq. (54) takes the form

$$
S_{\alpha \beta}=4 k_{B} T G_{\alpha \beta},
$$

which is familiar for the two-terminal case, $S=4 k_{B} T G$, with $G$ being the conductance. From Eq. (54) we see that the fluctuation spectrum of the mean squared current at a contact $\alpha$ is positive (since $G_{\alpha \alpha}>0$ ) but that 
the current-current correlations of the fluctuations at different probes are negative (since $G_{\alpha \beta}<0$ ). The sign of the equilibrium current-current fluctuations is independent of statistics: Intensity-intensity fluctuations for a system of bosons in which the electron reservoirs are replaced by black body radiators are also negative. We thus see that equilibrium noise does not provide any information of the system beyond that already known from conductance measurements.

Nevertheless, the equilibrium noise is important, if only to calibrate experiments and as a simple test for theoretical discussions. Experimentally, a careful study of thermal noise in a multi-terminal structure (a quantum Hall bar with a constriction) was recently performed by Henny et al [12]. Within the experimental accuracy, the results agree with the theoretical predictions.

Shot noise. Zero temperature. We now consider noise in a system of fermions 5 in a transport state. In the zero temperature limit the Fermi distribution in each reservoir is a step function $f_{\alpha}(E)=\theta\left(\mu_{\alpha}-E\right)$. Using this we can rewrite Eq. (52) as

$$
\begin{aligned}
S_{\alpha \beta}= & \frac{e^{2}}{2 \pi \hbar} \sum_{\gamma \neq \delta} \int d E \operatorname{Tr}\left[s_{\alpha \gamma}^{\dagger} s_{\alpha \delta} s_{\beta \delta}^{\dagger} s_{\beta \gamma}\right] \\
& \times\left\{f_{\gamma}(E)\left[1-f_{\delta}(E)\right]+f_{\delta}(E)\left[1-f_{\gamma}(E)\right]\right\} .
\end{aligned}
$$

We are now prepared to make two general statements. First, correlations of the current at the same lead, $S_{\alpha \alpha}$, are positive. This is easy to see, since their signs are determined by positively defined quantities $\operatorname{Tr}\left[s_{\alpha \gamma}^{\dagger} s_{\alpha \delta} s_{\alpha \delta}^{\dagger} s_{\alpha \gamma}\right]$. The second statement is that the correlations at different leads, $S_{\alpha \beta}$ with $\alpha \neq \beta$, are negative. This becomes clear if we use the property $\sum_{\delta} s_{\alpha \delta} s_{\beta \delta}^{\dagger}=0$ and rewrite Eq. (55) as

$$
\begin{aligned}
S_{\alpha \beta} & =-\frac{e^{2}}{\pi \hbar} \int d E \\
& \times \operatorname{Tr}\left[\left(\sum_{\gamma} s_{\beta \gamma} s_{\alpha \gamma}^{\dagger} f_{\gamma}(E)\right)\left(\sum_{\delta} s_{\alpha \delta} s_{\beta \delta}^{\dagger} f_{\delta}(E)\right)\right] .
\end{aligned}
$$

The integrand is now positively defined. Of course, current conservation implies that if all cross-correlations $S_{\alpha \beta}$ are negative for all $\beta$ different from $\alpha$, the spectral function $S_{\alpha \alpha}$ must be positive.

Actually, these statements are even more general. One can prove that cross-correlations in the system of fermions are generally negative at any temperature, see Ref. [9] for details. On the other hand, this is not correct for a system of bosons, where under certain conditions cross-correlations can be positive.

\footnotetext{
${ }^{5}$ For bosons at zero temperature one needs to take into account Bose condensation effects.

${ }^{6}$ These quantities are called "noise conductances" in Ref. 9].
}

Two-terminal conductors. Let us now consider the zero-temperature shot noise of a two-terminal conductor. Again we denote the leads as left (L) and right (R). Due to current conservation, we have $S \equiv S_{L L}=$ $S_{R R}=-S_{L R}=-S_{R L}$. Utilizing the representation of the scattering matrix (30), and taking into account that the unitarity of the matrix $s$ implies $r^{\dagger} r+t^{\dagger} t=1$, we obtain after some algebra

$$
S=\frac{e^{2}}{\pi \hbar} \operatorname{Tr}\left(r^{\dagger} r t^{\dagger} t\right) e|V|,
$$

where the scattering matrix elements are evaluated at the Fermi level. This is the basis invariant relation between the scattering matrix and the shot noise at zero temperature. Like the expression of the conductance, Eq. (38), we can express this result in the basis of eigen channels with the help of the transmission probabilities $T_{n}$ and reflection probabilities $R_{n}=1-T_{n}$,

$$
S_{L L}=\frac{e^{3}|V|}{\pi \hbar} \sum_{n} T_{n}\left(1-T_{n}\right) .
$$

We see that the non-equilibrium (shot) noise is not simply determined by the conductance of the sample. Instead, it is determined by a sum of products of transmission and reflection probabilities of the eigen channels. Only in the limit of low-transparency $T_{n} \ll 1$ in all eigen channels is the shot noise given by the Poisson value, discussed by Schottky,

$$
S_{P}=\frac{e^{3}|V|}{\pi \hbar} \sum_{n} T_{n}=2 e\langle I\rangle
$$

It is clear that zero-temperature shot noise is always suppressed in comparison with the Poisson valuel. In particular, neither closed $\left(T_{n}=0\right)$ nor open $\left(T_{n}=1\right)$ channels contribute to shot noise; the maximal contribution comes from channels with $T_{n}=1 / 2$. The suppression below the Poissonian limit given by Eq. (58) was one of the aspects of noise in mesoscopic systems which triggered many of the subsequent theoretical and experimental works. A convenient measure of sub-Poissonian shot noise is the Fano factor $F$ which is the ratio of the actual shot noise and the Poisson noise that would be measured if the system produced noise due to single independent electrons,

$$
F=\frac{S_{L L}}{S_{P}}
$$

\footnotetext{
${ }^{7}$ This statement is only valid for non-interacting systems. Interactions may cause instabilities in the system, driving the noise to super-Poissonian values. Noise in systems with multistable current-voltage characteristics (caused, for example, by a non-trivial structure of the energy bands, like in the Esaki diode) may also be super-Poissonian. These features are discussed in Section V.
} 
For energy independent transmission and/or in the linear regime the Fano factor is

$$
F=\frac{\sum_{n} T_{n}\left(1-T_{n}\right)}{\sum_{n} T_{n}}
$$

The Fano factor assumes values between zero (all channels are transparent) and one (Poissonian noise). In particular, for one channel it becomes $(1-T)$.

Unlike the conductance, which can be expressed in terms of (transmission) probabilities independent of the choice of basis, the shot noise, even for the two terminal conductors considered here, can not be expressed in terms of probabilities. The trace of Eq. (56) is a sum over $k, l, m, n$ of terms $r_{k n}^{*} r_{k m} t_{l m}^{*} t_{l n}$, which by themselves are not real valued if $m \neq n$ (in contrast to the Eq. (41) for the conductance). This is a signature that carriers from different quantum channels interfere and must remain indistinguishable. It is very interesting to examine whether it is possible to find experimental arrangements which directly probe such exchange interference effects, and we return to this question later on. In the remaining part of this subsection we will use the eigen channel basis which offers the most compact representation of the results.

The general result for the noise power of the current fluctuations in a two-terminal conductor is

$$
\begin{aligned}
S & =\frac{e^{2}}{\pi \hbar} \sum_{n} \int d E\left\{T_{n}(E)\left[f_{L}\left(1 \mp f_{L}\right)+f_{R}\left(1 \mp f_{R}\right)\right]\right. \\
& \left. \pm T_{n}(E)\left[1-T_{n}(E)\right]\left(f_{L}-f_{R}\right)^{2}\right\} .
\end{aligned}
$$

Here the first two terms are the equilibrium noise contributions, and the third term, which changes sign if we change statistics from fermions to bosons, is the nonequilibrium or shot noise contribution to the power spectrum. Note that this term is second order in the distribution function. At high energies, in the range where both the Fermi and Bose distribution function are well approximated by a Maxwell-Boltzmann distribution, it is negligible compared to the equilibrium noise described by the first two terms. According to Eq. (61) the shot noise term enhances the noise power compared to the equilibrium noise for fermions but diminishes the noise power for bosons.

In the practically important case, when the scale of the energy dependence of transmission coefficients $T_{n}(E)$ is much larger than both the temperature and applied voltage, these quantities in Eq. (61) may be replaced by their values taken at the Fermi energy. We obtain then (only fermions are considered henceforth)

$$
\begin{aligned}
S & =\frac{e^{2}}{\pi \hbar}\left[2 k_{B} T \sum_{n} T_{n}^{2}\right. \\
& \left.+e V \operatorname{coth}\left(\frac{e V}{2 k_{B} T}\right) \sum_{n} T_{n}\left(1-T_{n}\right)\right],
\end{aligned}
$$

where $V$ is again the voltage applied between the left and right reservoirs. The full noise is a complicated function of temperature and applied voltage rather than a simple superposition of equilibrium and shot noise? For low voltages $\mathrm{eV} \ll k_{B} T$ we obtain $S=4 k_{B} T G$, in accordance with the general result (54).

Note that, since $\operatorname{coth} x>1 / x$ for any $x>0$, the actual noise (62) for any voltage is higher than the equilibrium noise. This is not generally correct if the transmission coefficients are strongly energy dependent. As pointed out by Lesovik and Loosen [13], in certain situations (for instance, when the transmission coefficients sharply peak as functions of energy) the total non-equilibrium noise may be actually lower than the equilibrium noise at the same temperature.

We conclude this subsection with some historical remarks. Already Kulik and Omel'yanchuk [14 noticed that the shot noise in ballistic contacts (modeled as an orifice in an insulating layer between two metallic reservoirs) vanishes if there is no elastic impurity scattering. Subsequently, Khlus [15 considered such a point contact with elastic scattering and derived Eq. (62) by means of a Keldysh Green's function technique. The papers by Kulik and Omel'yanchuk and by Khlus remained unknown, they were either not or only poorly cited even in the Russian literature. Later Lesovik [16] derived Eq. (61) in the framework of the scattering approach (for the case of fermions). Independently, Yurke and Kochanski [17] investigated the momentum noise of a tunneling microscopic tip, also based on the scattering approach, treating only the one-channel case; Ref. [16] treated the multichannel case, but assumed at the outset that the scattering matrix is diagonal and that the diagonal channels are independent. A generalization for many-channel conductors described by an arbitrary scattering matrix (without assumption of independence) and for the many-terminal case was given in Refs. 18, 19. The same results were later discussed by Landauer and Martin [20] and Martin and Landauer [21] appealing to wave packets. The treatment of wave-packet overlap (see subsection II B) is avoided by assuming that wave packets are identical and timed to arrive at the same instant. Ref. [9], which we followed in this subsection, is a long version of the papers 18,19 .

\section{E. Voltage Fluctuations}

Role of external circuit. Thus far all the results which we have presented are based on the assumption that the sample is part of an external circuit with zero

\footnotetext{
${ }^{8}$ The full noise can be divided into equilibrium-like and transport parts, see Ref. [9. This division is, of course, arbitrary.
} 
impedance. In this case the voltage (voltages) applied to the sample can be viewed to be a fixed non-fluctuating quantity and the noise properties are determined by the current correlations which we have discussed. The idealized notion of a zero-impedance external circuit does often not apply. Fig. 3 shows a simple example: The sample $\mathrm{S}$ is part of an electrical circuit with resistance $R_{e x t}$ and a voltage source which generates a voltage $V_{e x t}$. (In general the external circuit is described by a frequency dependent impedance $Z_{\text {ext }}(\omega)$ ). As a consequence, in such a circuit, we deal with both current fluctuations and voltage fluctuations.

The current fluctuations through the sample are now governed by the fluctuations $\Delta V(t)$ of the voltage across the sample, which generate a fluctuating current $\Delta I_{V}(\omega)=G(\omega) \Delta V(\omega)$, where $G(\omega)$ is frequencydependent conductance (admittance) of the sample. In addition, there is the contribution of the spontaneous current fluctuations $\delta I(\omega)$ themselves. The total fluctuating current through the sample is thus given by

$$
\Delta I(\omega)=G(\omega) \Delta V(\omega)+\delta I(\omega) .
$$

Eq. (63) has the form of a Langevin equation with a fluctuating source term given by the spontaneous current fluctuations determined by the noise power spectrum $S$ of a two terminal conductor. To complete these equations we must now relate the current through the sample to the external voltage. The total current $I$ is related to the external voltage $V_{\text {ext }}$ and the voltage across the sample by the Kirchoff law $V+R_{\text {ext }} I=V_{\text {ext }}$. Here $V_{\text {ext }}$ is a constant, and the voltage and current fluctuations are thus related by $\Delta V+R_{e x t} \Delta I=0$, or

$$
\Delta V(\omega)=-R_{e x t} \Delta I(\omega) .
$$

For $R_{e x t}=0$ (zero external impedance) we have the case of a voltage controlled external circuit, while for $R_{\text {ext }} \rightarrow \infty$ (infinite external impedance) we have the case of a current controlled external circuit. The Langevin approach assumes that the mesoscopic sample and the external circuit can be treated as separate entities, each of which might be governed by quantum effects, but that there are no phase coherent effects which would require the treatment of the sample and the circuit as one quantum mechanical entity. In such a case the distinction between sample and external circuit would presumably be meaningless. Eliminating the voltage fluctuations in Eqs. (63) and (64) gives for the current fluctuations $\Delta I(\omega)\left(1+G(\omega) R_{\text {ext }}\right)=\delta I(\omega)$ and with the resistance of the sample $R=1 / G(0)$ and the noise power spectrum $S$ we obtain in the zero-frequency limit 9

\footnotetext{
${ }^{9}$ The quantity $S_{I I}$ is defined by Eq.(49), as before. The quantity $S_{V V}$ is defined by the same expression where current fluctuations $\Delta I$ are replaced by the voltage fluctuations $\Delta V$.
}

$$
S_{I I}=\frac{S}{\left(1+R_{e x t} / R\right)^{2}} .
$$

Eq. (65) shows that the external impedance becomes important if it is comparable or larger than the resistance of the sample. Eliminating the current we obtain $\left(1 / R_{e x t}+G(\omega)\right) \Delta V(\omega)=-\delta I(\omega)$, and thus a voltage fluctuation spectrum given by

$$
S_{V V}=\frac{S}{\left(\frac{1}{R_{e x t}}+\frac{1}{R}\right)^{2}} .
$$

At equilibrium, where the current noise power is given by $S=4 k_{B} T G$, Eq. (56) gives $S_{V V}=4 k_{B} T / R\left(1 / R_{\text {ext }}+\right.$ $1 / R)^{2}$ which reduces in the limit $R_{e x t} \rightarrow \infty$ to the familiar Johnson-Nyquist result $S_{V V}=4 k_{B} T R$ for the voltage fluctuations in an infinite external impedance circuit. The procedure described above can also be applied to shot noise as long as we are only concerned with effects linear in the voltage $V$. Far from equilibrium, this approach applies if we replace the conductance (resistance) by the differential conductance (resistance) and if a linear fluctuation theory is sufficient.

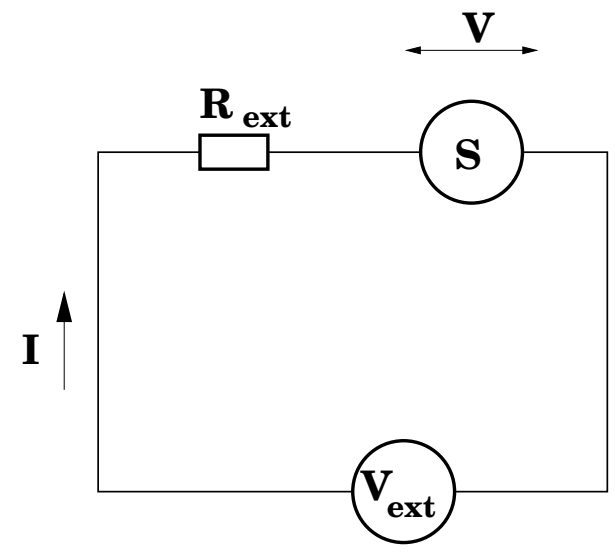

FIG. 3. Noise measurements in an external circuit. The sample is denoted $S$.

External circuit: multi-probe conductors. For a multi-probe geometry the consideration of the external circuit is similarly based on the Langevin equation [9],

$$
\Delta I_{\alpha}=\sum_{\beta} G_{\alpha \beta} \Delta V_{\beta}+\delta I_{\alpha},
$$

where $G_{\alpha \beta}$ is an element of the conductance matrix and $\delta I_{\alpha}$ is a fluctuating current with the noise power spectrum $S_{\alpha \beta}$. The external circuit loops connecting to a multi-probe conductor can have different impedances: the external impedance is thus also represented by a matrix which connects voltages and currents at the contacts of the multi-probe conductor. Ideally, the current source and sink contacts are connected to a zero impedance external circuit, whereas the voltage probes are connected to an external loop with infinite impedance. 
At equilibrium, in the limiting case that all probes are connected to infinite external impedance loops, the voltage fluctuations can be expressed in terms of multi-probe resistances. Consider first a four-probe conductor. A four-probe resistance is obtained by injecting current in contact $\alpha$ taking it out at contact $\beta$ and using two additional contacts $\gamma$ and $\delta$ to measure the voltage difference $V_{\gamma}-V_{\delta}$. The four-probe resistance is defined as $R_{\alpha \beta, \gamma \delta}=\left(V_{\gamma}-V_{\delta}\right) / I$. Using the conductance matrix of a four-probe conductor, a little algebra shows that,

$$
R_{\alpha \beta, \gamma \delta}=D^{-1}\left(G_{\gamma \alpha} G_{\delta \beta}-G_{\gamma \beta} G_{\delta \alpha}\right)
$$

where $D$ is any sub-determinant of rank three of the conductance matrix. (Due to current conservation and gauge invariance all possible subdeterminants of rank three of the conductance matrix are identical and are even functions of the applied magnetic field). Eqs. (68) can be applied to a conductor with any number of contacts larger than four, since the conductance matrix of any dimension can be reduced to a conductance matrix of dimension four, if the additional contacts not involved in the measurement are taken to be connected to infinite external impedance loops. Similarly, there exists an effective conductance matrix of dimension three which permits to define a three-probe measurement. In such a measurement one of the voltages is measured at the current source contact or current sink contact and thus two of the indices in $R_{\alpha \beta, \gamma \delta}$ are identical, $\alpha=\gamma$ or $\beta=\delta$. Finally, if the conductance matrix is reduced to a $2 \times 2$ matrix, we obtain a resistance for which two pairs of indices are identical, $R_{\alpha \beta, \alpha \beta}$ or $R_{\alpha \beta, \beta \alpha}=-R_{\alpha \beta, \alpha \beta}$. With these resistances we can now generalize the familiar Johnson-Nyquist relation $S_{V V}=4 k_{B} T R$ for two-probe conductors, to the case of a multi-probe conductor. For the correlation of a voltage difference $V_{\alpha}-V_{\beta}$ measured between contacts $\alpha$ and $\beta$ with a voltage fluctuation $V_{\gamma}-V_{\delta}$ measured between contacts $\gamma$ and $\delta$ Eq. (67) leads to

$$
\left\langle\left(V_{\alpha}-V_{\beta}\right)\left(V_{\gamma}-V_{\delta}\right)\right\rangle=2 k_{B} T\left(R_{\alpha \beta, \gamma \delta}+R_{\gamma \delta, \alpha \beta}\right) .
$$

The mean squared voltage fluctuations $\alpha=\gamma$ and $\beta=\delta$ are determined by the two-terminal resistances $R_{\alpha \beta, \alpha \beta}$ of the multi-probe conductor. The correlations of voltage fluctuations (in the case when all four indices differ) are related to symmetrized four-probe resistances.

If shot noise is generated, for instance, by a current incident at contact $\alpha$ and taken out at contact $\beta$ (in a zero external impedance loop) and with all other contacts connected to an infinite impedance circuit, the voltage fluctuations are [22]

$$
\left\langle\left(\delta V_{\gamma}-\delta V_{\delta}\right)\left(\delta V_{\epsilon}-\delta V_{\zeta}\right)\right\rangle=\sum_{\alpha \eta} R_{\alpha \beta, \epsilon \zeta} R_{\eta \beta, \gamma \delta} S_{\alpha \eta}
$$

where $S_{\alpha \eta}$ is the noise power spectrum of the current correlations at contacts $\alpha$ and $\eta$, and $\beta$ is an arbitrary index.
These examples demonstrate that the fluctuations in a conductor are in general a complicated expression of the noise power spectrum determined for the zero-impedance case, the resistances (or far from equilibrium the differential resistances) and the external impedance (matrix). These considerations are of importance since in experiments it is the voltage fluctuations which are actually measured and which eventually are converted to current fluctuations.

\section{F. Applications}

In this subsection, we give some simple applications of the general formulae derived above, and illustrate them with experimental results. We consider only zero frequency limit. As we explained in the Introduction, we do not intend to give here a review of all results concerning a specific system. Instead, we focus on the application of the scattering approach. For results derived for these systems with other methods, the reader is addressed to Table 1.

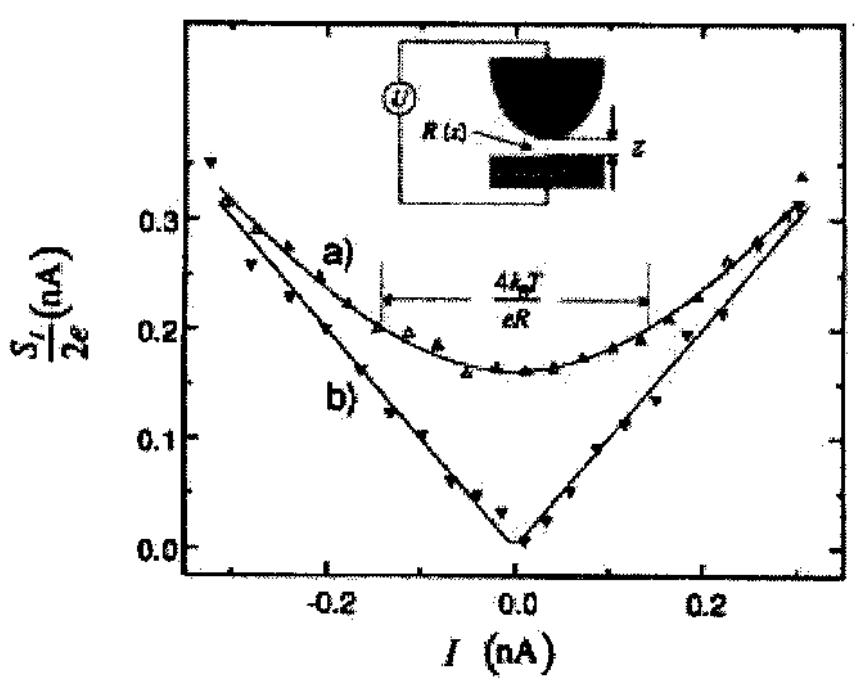

FIG. 4. Crossover from thermal to shot noise measured by Birk, de Jong, and Schönenberger 30]. Solid curves correspond to Eq. (71); triangles show experimental data for the two samples, with lower (a) and higher (b) resistance.

\section{Tunnel barriers}

For a tunnel barrier, which can be realized, for example, as a layer of insulator separating two normal metal electrodes, all the transmission coefficients $T_{n}$ are small, $T_{n} \ll 1$ for any $n$. Separating terms linear in $T_{n}$ in Eq. (62) and taking into account the definition of the Poisson noise, Eq.58, we obtain 


$$
S=\frac{e^{3} V}{\pi \hbar} \operatorname{coth}\left(\frac{e V}{2 k_{B} T}\right) \sum_{n} T_{n}=\operatorname{coth}\left(\frac{e|V|}{2 k_{B} T}\right) S_{P}
$$

At a given temperature, Eq. (71) describes the crossover from thermal noise at voltages $e|V| \ll k_{B} T$ to shot noise at voltages $e|V| \gg k_{B} T$. The transition is independent from any details of the tunnel barrier and occurs at $e|V|=k_{B} T$.

Eq. (71) is also obtained in the zero-frequency, zero charging energy limit of microscopic theories of low transparency normal tunnel junctions or Josephson junctions 23 28]. These theories employ the tunneling Hamiltonian approach and typically only keep the terms of lowest non-vanishing order in the tunneling amplitude. Poissonian shot noise was measured experimentally in semiconductor diodes, see e.g. Ref. [29]; these devices, however, could hardly be called mesoscopic, and it is not always easy to separate various sources of noise. More recently, Birk, de Jong, and Schönenberger [30] presented measurements of noise in a tunnel barrier formed between an STM tip and a metallic surface. Specifically addressing the crossover between the thermal and shot noise, they found an excellent agreement with Eq. (71). Their experimental results are shown in Fig. 4 .

\section{Quantum point contacts}

A point contact is usually defined as a constriction between two metallic reservoirs. Experimentally, it is typically realized by depleting of a two-dimensional electron gas formed with the help of a number of gates. Changing the gate voltage $V_{g}$ leads to the variation of the width of the channel, and consequently of the electron concentration. All the sizes of the constriction are assumed to be shorter than the mean free path due to any type of scattering, and thus transport through the point contact is ballistic. In a quantum point contact the width of the constriction is comparable to the Fermi wavelength.

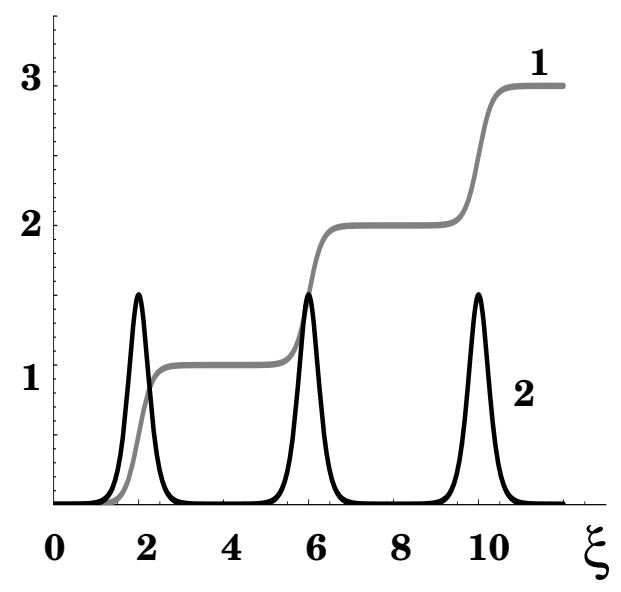

FIG. 5. Conductance in units of $e^{2} / 2 \pi \hbar$ (curve 1) and zero-frequency shot noise power in units of $e^{3}|V| / 6 \pi \hbar$ (curve 2) for a quantum point contact with $\omega_{y}=4 \omega_{x}$ as a function of the gate voltage. Here $\xi=\left(E_{F}-V_{0}\right) / \hbar \omega_{x}$ is a dimensionless energy.

Quantum point contacts have drawn wide attention after experimental investigations [31,32] showed steps in the dependence of the conductance on the gate voltage. This stepwise dependence is illustrated in Fig. 5, curve 1. An explanation was provided by Glazman et al [33], who modeled the quantum point contact as a ballistic channel between two infinitely high potential walls (Fig. 6a). If the distance between the walls $d(x)$ (width of the contact) is changing slowly in comparison with the wavelength, transverse and longitudinal motion can be approximately separated. The problem is then effectively reduced to one-dimensional motion in the adiabatic potential $U(x)=\pi^{2} n^{2} \hbar^{2} / 2 m d^{2}(x)$, which depends on the width profile and the the transverse channel number $n$. Changing the gate voltage leads to the modification of the potential profile. Theoretically it is easier to fix the geometry of the sample, i.e the form of the potential, and vary the Fermi energy in the channel $E_{F}$ (Fig. 6b). The external potential is smooth, and therefore may be treated semi-classically. This means that the channels with $n<k_{F} d_{\min } / \pi$ (here $\hbar k_{F} \equiv\left(2 m E_{F}\right)^{1 / 2}$, and $d_{\text {min }}$ is the minimal width of the contact) are open and transparent, $T_{n}=1$, while the others are closed, $T_{n}=0$. The conductance (40) is proportional to the number of open channels and therefore exhibits plateaus as a function of the gate voltage. At the plateaus, shot noise is equal to zero, since all the channels are either open or closed. The semi-classical description fails when the Fermi energy lies close to the top of the potential in one of the transverse channels. Then the transmission coefficient for this channel increases from zero to one due to quantum tunneling through the barrier and quantum reflection at the barrier. The transition from one plateau to the next is associated with a spike in the shot noise as we will now discuss.

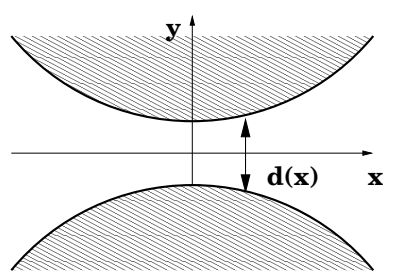

a)

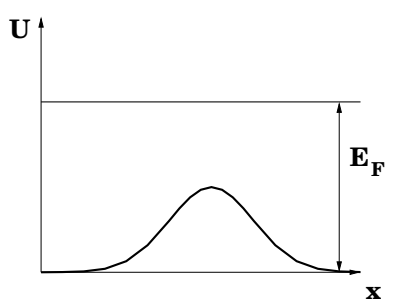

b)
FIG. 6. Geometry of the quantum point contact in the hard-wall model (a) and the effective potential for one-dimensional motion (b).

A more realistic description of the quantum point contact takes into account that the potential in the transverse direction $y$ is smooth [34]. The constriction can 
then be thought of as a bottleneck with an electrostatic potential of the form of a saddle. Quite generally the potential can be expanded in the directions away from the center of the constriction,

$$
V(x, y)=V_{0}-\frac{1}{2} m \omega_{x}^{2} x^{2}+\frac{1}{2} m \omega_{y}^{2} y^{2},
$$

where the constant $V_{0}$ denotes the potential at the saddle point. Experimentally it is a function of the gate voltage. The transmission probabilities are given by Ref. [34],

$$
\begin{aligned}
T_{n}(E) & =\left[1+\exp \left(-\pi \epsilon_{n}\right)\right]^{-1}, \\
\epsilon_{n} & \equiv 2\left[E-\hbar \omega_{y}\left(n+\frac{1}{2}\right)-V_{0}\right] / \hbar \omega_{x} .
\end{aligned}
$$

The transmission probability $T_{n}(E)$ exhibits a crossover from zero to one as the energy $E$ passes the value $V_{0}+$ $\hbar \omega_{y}(n+1 / 2)$. The resulting zero temperature shot noise as a function of $V_{0}$, using Eq. (57), is illustrated in Fig. 5 for the case $\omega_{y}=4 \omega_{x}$ (curve 2). The conductance of this quantum point contact is shown in Fig. 5 as curve 1. As expected, the shot noise dependence is a set of identical spikes between the plateaus. The height of each spike is $e^{3}|V| / 4 \pi \hbar$ up to exponential accuracy. At the plateaus shot noise is exponentially suppressed. This behavior of shot noise in a quantum point contact was predicted by Lesovik [16]. The shot noise of a saddle point model of a quantum point contact was presented in Ref. [1]. Scherbakov et al [35] thoroughly analyze and compare shot noise for various models of quantum point contacts. Using a classical (master equation) approach, shot noise suppression was also confirmed by Chen and Ying [36].

If a magnetic field is applied in the transverse direction, the energies $\epsilon_{n}$ are pushed up. Shot noise is thus an oscillating function of the magnetic field for a fixed gate voltage. Strong magnetic fields may even drive the quantum point contact to the regime $E_{F}<\epsilon_{0}$, suppressing the shot noise completely [35].

Using Eq. (61), it is easy also to study shot noise in the non-linear regime as the function of the applied bias voltage $V$. We obtain

$$
S \approx \frac{e^{2} \omega_{x}}{(2 \pi)^{2}} N_{V}, \quad N_{V}=\frac{e|V|}{\hbar \omega_{y}},
$$

where $N_{V}$ is the number of channels which open in the energy interval between zero and $e|V|$. Eq. (73) applies when this number is large, $N_{V} \gg 1$. For even higher voltages $e|V|>V_{0}$, the noise becomes voltage independent, as found by Larkin and Reznikov [37]. They also discuss self-consistent interactions and found that the non-linear shot noise is suppressed as compared to the non-interacting value.

As the number of open channels becomes large, so that the width of the constriction is much wider than the Fermi wavelength (classical point contact), the shot noise stays the same, while the conductance grows proportional to the number of channels. Thus, shot noise becomes small in comparison with the Poisson value (at the top of the $n$-th spike this suppression equals $(4 n)^{-1}$ ), and in this sense shot vanishes for a classical point contact, as found by Kulik and Omel'yanchuk [14]. Note, however, that the shot noise really disappears only when inelastic scattering becomes significant (see subsection $\amalg \mathrm{IG}$ ).

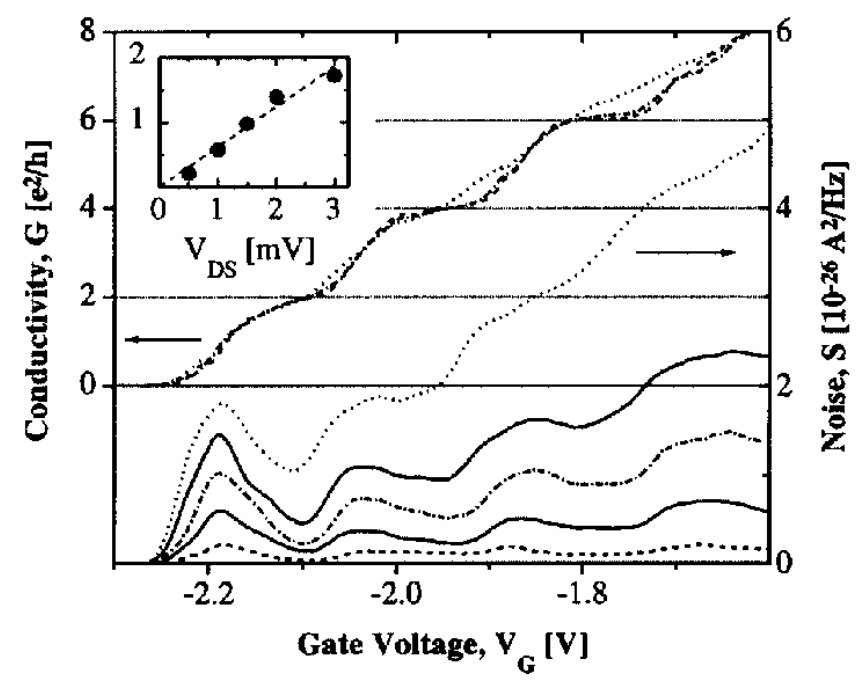

FIG. 7. Conductance (upper plot) and shot noise (lower plot) as functions of the gate voltage, as measured by Reznikov et al [42]. Different curves correspond to five different bias voltages.

Experimentally, sub-Poissonian shot noise suppression was observed by Akimenko, Verkin, and Yanson [38] in a slightly different system, a metallic quantum point contact, which is essentially an orifice in a thin insulating layer between two metallic reservoirs. In this system, however, it is difficult to separate different sources of noise. In ballistic quantum point contacts sub-Poisson suppression was observed in an early experiment by $\mathrm{Li}$ et al 39], and later by Dekker et al [40,41. Reznikov et al [42] found clearly formed peaks in the shot noise as a function of the gate voltage. A considerable improvement in the experimental technique was obtained by measuring noise in the $\mathrm{MHz}$ range at frequencies far above the range where $1 / f$-noise contributes. The results of Reznikov et al [42] are shown in Fig. 7. Compared to the theory the experimental noise peaks exhibit a slight asymmetry around the transition point. Kumar et al 433] developed a different low frequency technique based on voltage correlation measurements to filter out unwanted noise. They found that "the agreement /of experimental results〉 with theoretical expectations, within the calculable statistical deviations, is nearly perfect". Recently, van den Brom and van Ruitenbeek 44 demonstrated that shot noise measurements can be used to extract information on the transmission probabilities of the eigen channels of nanoscopic metallic point contacts. Subsequently, Bürki and Stafford [45] were able to reproduce their results quantitatively based on a simple theoretical model which takes into account only two features of the 
contacts, the confinement of electrons and the coherent backscattering from imperfections.

\section{Resonant tunnel barriers}

The transport through two consecutive tunnel barriers allows already to discuss many aspects of shot noise suppression. Let us first consider the case of purely onedimensional electron motion through two potential barriers with transmission probabilities $T_{L}$ and $T_{R}$, separated by a distance $w$, as shown in Fig. 8. Eventually, we will assume that the transmission of each barrier is low, $T_{L} \ll 1$ and $T_{R} \ll 1$. An exact expression for the transmission coefficient of the whole structure is

$$
=\frac{T(E)}{1+\left(1-T_{L}\right)\left(1-T_{R}\right)-2 \sqrt{\left(1-T_{L}\right)\left(1-T_{R}\right)} \cos \phi(E)},
$$

with $\phi(E)$ being the phase accumulated during motion between the barriers; in our particular case $\phi(E)=$ $2 w(2 m E)^{1 / 2} / \hbar$. Eq. (74) has a set of maxima at the resonant energies $E_{n}^{r}$ such that the phase $\phi\left(E_{n}^{r}\right)$ equals $2 \pi n$. Expanding the function $\phi(E)$ around $E_{n}^{r}$, and neglecting the energy dependence of the transmission coefficients, we obtain the Breit-Wigner formula [46,47]

$$
T(E)=T_{n}^{\max } \frac{\Gamma_{n}^{2} / 4}{\left(E-E_{n}^{r}\right)^{2}+\Gamma_{n}^{2} / 4}, \quad T_{n}^{\max }=\frac{4 \Gamma_{L n} \Gamma_{R n}}{\Gamma_{n}^{2}} .
$$

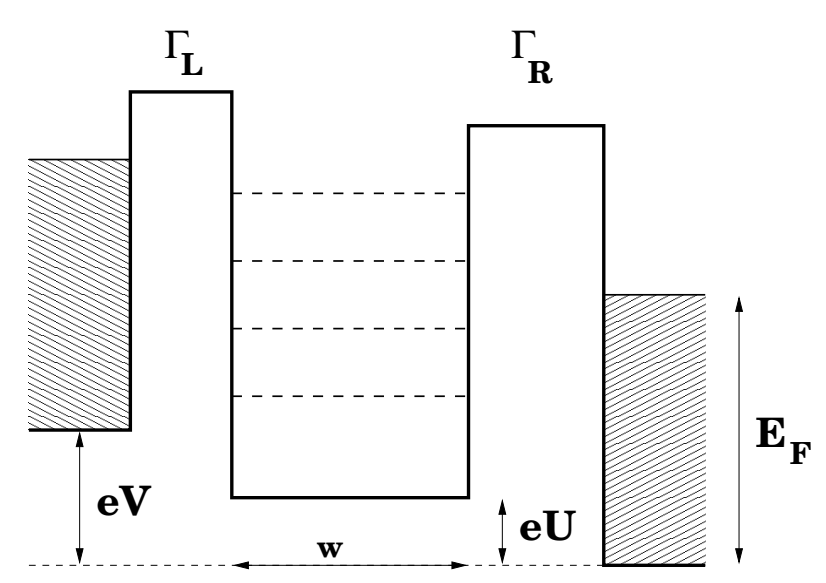

FIG. 8. Resonant double barriers. The case of low voltage is illustrated; resonant levels inside the well are indicated by dashed lines.

$T_{n}^{\max }$ is the maximal transmission probability at resonance. We have introduced the partial decay widths $\Gamma_{L n, R n}=\hbar \nu_{n} T_{L, R}$. The attempt frequency $\nu_{n}$ of the $n$-th resonant level is given by $\nu_{n}^{-1}=(\hbar / 2)\left(d \phi / d E_{n}^{r}\right)=w / v_{n}$, $v_{n}=\left(2 E_{n}^{r} / m\right)^{1 / 2} . \quad \Gamma_{n} \equiv \Gamma_{L n}+\Gamma_{R n}$ is the total decay width of the resonant level. Eq. (75) is, strictly speaking, only valid when the energy $E$ is close to one of the resonant energies $\sqrt{10} E_{n}^{r}$. In many situations, however, the Lorentz tails of $T(E)$ far from the resonances are not important, and one can write

$$
T(E)=\sum_{n} T_{n}^{\max } \frac{\Gamma_{n}^{2} / 4}{\left(E-E_{n}^{r}\right)^{2}+\Gamma_{n}^{2} / 4} .
$$

Despite the fact that the transparencies of both barriers are low, we see that the total transmission coefficient shows sharp peaks around resonant energies. This effect is a consequence of constructive interference and is known as resonant tunneling. The transmission coefficient at the top of a peak equals $T_{n}^{\max }$; for a symmetric resonance $\Gamma_{L n}=\Gamma_{R n}$ the transmission is ideal, $T_{n}^{\max }=1$. This dependence may be probed 11 by applying a gate voltage. The gate voltage moves the positions of the resonant levels, and the conductance exhibits peaks around each resonance.

In the linear regime the shot noise is determined by the transmission coefficient evaluated at the Fermi level, and is thus an oscillating function of the gate voltage, vanishing almost completely between the peaks. The Fano factor (60) at the top of each peak is equal to $F=\left(\Gamma_{L n}-\Gamma_{R n}\right)^{2} / \Gamma_{n}^{2}$. It vanishes for a symmetric barrier. For a resonance with $T_{n}^{\max }>1 / 2$ the Fano factor reaches a maximum each time when the transmission probability passes through $T=1 / 2$; for a resonance with $T_{n}^{\max }<1 / 2$ the shot noise is maximal at resonance.

One-dimensional problem, non-linear regime. For arbitrary voltage, direct evaluation of the expressions (39) and (61) gives an average current,

$$
I=\frac{e}{\hbar} \sum_{n=1}^{N_{V}} \frac{\Gamma_{L n} \Gamma_{R n}}{\Gamma_{n}}
$$

and a zero-temperature shot noise,

$$
S=\frac{2 e^{2}}{\hbar} \sum_{n=1}^{N_{V}} \frac{\Gamma_{L n} \Gamma_{R n}\left(\Gamma_{L n}^{2}+\Gamma_{R n}^{2}\right)}{\Gamma_{n}^{3}}
$$

Here $N_{V}$ is the number of resonant levels in the energy strip $e|V|$ between the chemical potentials of the left and right reservoirs. Eqs. (76) and (77) are only valid when this number is well defined - the energy difference between any resonant level and the chemical potential of any reservoir must be much greater than $\Gamma$. Under this condition both the current and the shot noise are independent of the applied voltage. The dependence of both the current and the shot noise on the bias voltage $V$ is thus a set of plateaus, the height of each plateau being

\footnotetext{
${ }^{10}$ We assume that the resonances are well separated, $\Gamma \ll$ $\hbar^{2} / 2 m w^{2}$.

${ }^{11}$ For discussion of experimental realizations, see below.
} 
proportional to the number of resonant levels through which transmission is possible. Outside this regime, when one of the resonant levels is close to the chemical potential of left and/or right reservoir, a smooth transition with a width of order $\Gamma$ from one plateau to the next occurs.

Consider now for a moment, a structure with a single resonance. If the applied voltage is large enough, such that the resonance is between the Fermi level of the source contact and that of the the sink contact, the Fano factor is

$$
F=\frac{\Gamma_{L}^{2}+\Gamma_{R}^{2}}{\Gamma^{2}} .
$$

It varies between $1 / 2$ (symmetric barrier) and 1 (very asymmetric barrier). Expression (78) was obtained by Chen and Ting [48] using a nonequilibrium Green's functions technique, and independently in Ref. [19] using the scattering approach. It was confirmed in Monte Carlo simulations performed by Reklaitis and Reggiani [49,50]. If the width of the resonance is comparable to the applied voltage, Eq. (78) has to be supplemented by correction terms due to the Lorentz tails of the Breit-Wigner formula, as found in Ref. [19] and later by Averin [51].

It is also worthwhile to point out that our quantummechanical derivation assumes that the electron preserves full quantum coherence during the tunneling process (coherent tunneling model). Another limiting case occurs when the electron completely loses phase coherence once it is inside the well (sequential tunneling model). This latter situation can be described both classically (usually, by means of a master equation) and quantum-mechanically (e.g., by connecting to the well one or several fictitious voltage probes which serve as "dephasing" leads). These issues are addressed in Section V, where we show that the result for the Fano factor Eq. (78) remains independent of whether we deal with a coherent process or a fully incoherent process. The Fano factor Eq. (78) is thus insensitive to dephasing.

Quantum wells. The double-barrier problem is also relevant for quantum wells, which are two- or threedimensional structures 12 , consisting of two planar (linear in two dimensions) potential barriers. Of interest is transport in the direction perpendicular to the barriers (across the quantum well, axis $z$ ). These systems have drawn attention already in the seventies, when resonant tunneling was investigated both theoretically [52] and experimentally [53].

If the area of the barriers (in the plane $x y) \mathcal{A}$ is very large, the summation over the transverse channels in Eqs. (39), (61) can be replaced by integration, and we obtain for the average current

\footnotetext{
${ }^{12}$ For simplicity, we use a three-dimensional notation. Specialization to two dimensions is trivial.
}

$$
\begin{aligned}
I & =\frac{e \nu_{2} \mathcal{A}}{2 \pi \hbar} \int_{0}^{\infty} d E_{\perp} d E_{z} T\left(E_{z}\right) \\
& \times\left\{f_{L}\left(E_{z}+E_{\perp}\right)-f_{R}\left(E_{z}+E_{\perp}\right)\right\}
\end{aligned}
$$

and the shot noise

$$
\begin{aligned}
S & =\frac{e^{2} \nu_{2} \mathcal{A}}{\pi \hbar} \int_{0}^{\infty} d E_{\perp} d E_{z} T\left(E_{z}\right)\left[1-T\left(E_{z}\right)\right] \\
& \times\left\{f_{L}\left(E_{z}+E_{\perp}\right)-f_{R}\left(E_{z}+E_{\perp}\right)\right\}
\end{aligned}
$$

with $\nu_{2}=m / 2 \pi \hbar^{2}$ the density of states of the twodimensional electron gas (per spin). The key point is that the transmission coefficient depends only on the energy of the longitudinal ${ }^{[3}$ motion $E_{z}$, and thus is given by the solution of the one-dimensional double-barrier problem, discussed above. Denoting $\mu_{L}=E_{F}+e V, \mu_{R}=E_{F}$, and integrating over $d E_{\perp}$, we write (the temperature is set to zero)

$$
\begin{aligned}
I & =\frac{e \nu_{2} \mathcal{A}}{2 \pi \hbar}\left\{e V \int_{0}^{E_{F}} d E_{z} T\left(E_{z}\right)\right. \\
& \left.+\int_{E_{F}}^{E_{F}+e V} d E_{z}\left(E_{F}+e V-E_{z}\right) T\left(E_{z}\right)\right\}
\end{aligned}
$$

and

$$
\begin{aligned}
S & =\frac{e^{2} \nu_{2} \mathcal{A}}{\pi \hbar}\left\{e V \int_{0}^{E_{F}} d E_{z} T\left(E_{z}\right)\left[1-T\left(E_{z}\right)\right]\right. \\
& \left.+\int_{E_{F}}^{E_{F}+e V} d E_{z}\left(E_{F}+e V-E_{z}\right) T\left(E_{z}\right)\left[1-T\left(E_{z}\right)\right]\right\} .
\end{aligned}
$$

Expressions (79) and (80) are valid in the linear and non-linear regimes, provided interactions are not important. We will consider the noise in the non-linear regime in Section V, where it will be shown that effects of charging of the well may play an important role. Here, specializing on the regime linear in the bias voltage $V$, we obtain a current $\langle I\rangle=G V$ determined by the conductance

$$
G=\frac{e^{2} \nu_{2} \mathcal{A} N_{F}}{\hbar} \frac{\Gamma_{L} \Gamma_{R}}{\Gamma},
$$

and a shot noise power

$$
S=2 e \frac{\left(\Gamma_{L}^{2}+\Gamma_{R}^{2}\right)}{\Gamma^{2}}\langle I\rangle .
$$

Here $N_{F}$ is the number of resonant states in the onedimensional problem, which lie below $E_{F}$. Eqs. 81) and (32) are valid only when the distance between all resonant levels and the Fermi level is much greater that $\Gamma$. This dependence may be probed again, like in a onedimensional structure, with the help of a gate. Both the

\footnotetext{
${ }^{13}$ Along the current, not along the well.
} 
current and the shot noise exhibit plateaus as a function of gate voltage; these plateaus are smoothly joined over a width of order $\Gamma$. This dependence resembles that of the non-linear one-dimensional regime, but it clearly is a consequence of different physics. Nevertheless, in the plateau regime the Fano factor is the same, Eq. (78). This fact was noted by Davis et al [54], who presented both quantum and classical derivations of this result. Classical theories of shot noise suppression in quantum wells are discussed in Section $\mathrm{V}$.

Averaging. Another point of view was taken by Melsen and Beenakker [55] and independently by Melnikov [56], who investigated not a single resonant tunneling structure but an ensemble of systems. Imagine an ensemble of quasi-one-dimensional double-barrier systems, in which some parameter is random. For definiteness, we assume that the systems are subject to a random gate voltage. Then in some of them the Fermi level is close to one of the resonant energies, and in others it lies between two resonant levels. Therefore, on average, shot noise must be finite even in the linear regime. To quantify this argument, we turn to the exact expression (74) for the transmission coefficient ${ }^{14}$ and assume that the phase $\phi$ is a random variable, uniformly distributed on the interval $(0,2 \pi)$. We only consider the linear regime.

First, we calculate the conductance,

$$
G=\frac{e^{2}}{2 \pi \hbar} \sum_{n} T_{n}=\frac{e^{2} N}{(2 \pi)^{2} \hbar} \int_{0}^{2 \pi} T(\phi) d \phi=\frac{e^{2} N}{2 \pi \hbar} \frac{T_{L} T_{R}}{T_{L}+T_{R}}
$$

where $N$ is the number of transverse channels, and we have taken into account $T_{L} \ll 1, T_{R} \ll 1$. The same calculation for the shot noise yields [56,1]

$$
\begin{aligned}
S & =\frac{e^{3}|V| N}{2 \pi^{2} \hbar} \int_{0}^{2 \pi} T(\phi)[1-T(\phi)] d \phi \\
& =\frac{e^{3}|V| N}{\pi \hbar} \frac{T_{L} T_{R}\left(T_{L}^{2}+T_{R}^{2}\right)}{\left(T_{L}+T_{R}\right)^{3}} .
\end{aligned}
$$

The Fano factor is given again by Eq. (78).

We can learn two lessons from this simple model. First, the Fano factor $(78) F=\left(\Gamma_{L}^{2}+\Gamma_{R}^{2}\right) / \Gamma^{2}$ appears each time when there is some kind of averaging in the system which involves one-dimensional motion across two barriers. In the two examples we considered, the onedimensional non-linear problem, and the quantum well in the linear regime, this averaging is provided by the summation over all the levels between the chemical potentials of the two reservoirs (one dimension), or the summation over the transverse channels at the given total energy

\footnotetext{
${ }^{14}$ The Breit-Wigner formula 75 ) cannot be used for this purpose, since it is not exact far from the resonance.
}

(quantum well). Thus, both problems prove to be selfaveraging. At the same time, this averaging is absent in the one-dimensional linear problem, and the Fano factor has nothing to do with Eq. (78) even between the resonances.

The second lesson is provided by the distribution function of the transmission coefficients of the eigen channels in the one-dimensional problem 55,56. Without giving details, we mention only that it has a bimodal form. The transmission coefficients assume values between $T_{\min }=T_{L} T_{R} / 4 \ll 1$ and $T_{\max }$; those close to $T_{\min }$ and $T_{\max }$ have higher probability than those lying in between. The Fano factor is very sensitive to the appearance of transmission coefficients close to one, since it is these values which cause the sub-Poisson suppression. Thus, for a symmetric barrier $T_{\max }=1$, and the probability to find the transmission coefficient close to 1 is high. This yields the lowest possible Fano factor $1 / 2$ which is possible in this situation. We will return in more detail to the distribution of transmission probabilities for metallic diffusive conductors and chaotic cavities.

Related work. Here we mention briefly additional theoretical results on noise in double-barrier and similar structures.

Runge [57 investigates noise in double-barrier quantum wells, allowing for elastic scattering inside the well. He employs a non-equilibrium Green's function technique and a coherent potential approximation, and arrives at rather cumbersome expressions for the average current and noise power. In the limit of zero temperature, however, his results yield the same Fano factor (78), despite the fact that both current and noise are sensitive to impurity scattering.

Lund B $\varnothing$ and Galperin [58,59 consider a resonant quantum well in a strong magnetic field perpendicular to the interfaces (along the axis $z$ ). They find that the shot noise power (in the linear and nonlinear regimes, but without charging effects taken into account) shows peaks each time when the new Landau level in the well crosses the chemical potential in the right reservoir.

Xiong 60] analyzes noise in superlattices of finite size (several consecutive barriers) using the transfer matrix method. His numerical results clearly show shot noise suppression with respect to the Poisson value, but, unfortunately, the Fano factor is not plotted.

Resonant tunneling through localized states. The following problem was discussed by Nazarov and Struben 61]. Consider now non-linear transport through one, one-dimensional, symmetric barrier, situated in the region $-w / 2<z<w / 2$. We assume that there are resonant states which are randomly distributed inside the barrier and strongly localized. Applying the model suggested for this situation by Larkin and Matveev [62], we assume that these resonant states are provided by impurities inside the barrier; the localization radius of each state is denoted by $\xi, \xi \ll w$. Transition rates are exponentially sensitive to the position of these impurities inside the barrier. In the regime of low impurity concentration, 
only those situated close to the center of the barrier contribute to the transport properties. Thus, the problem is effectively mapped onto a double-barrier problem, where the impurity region near the center of the barrier serves as a potential well, and is separated by two "barriers" from the left and right reservoirs. The tunneling rates through these "barriers" to a resonant state depend on the position $z$ of the impurity which provides this resonant state. We have 62

$$
\Gamma_{L, R}(z)=\Gamma_{0} \exp [ \pm z / \xi], \quad|z|<w / 2
$$

We have assumed that this amplitude is energy independent, and that impurities are uniformly distributed in energy and space. Thus, our expressions (76) and (77) hold, and must be averaged over impurity configurations. We write

$$
I=\frac{e}{\hbar} n_{0} \Gamma_{0} \int_{-\infty}^{\infty} \frac{d z}{2 \cosh (z / \xi)}
$$

and

$$
S=\frac{2 e^{2}}{\hbar} n_{0} \Gamma_{0} \int_{-\infty}^{\infty} d z \frac{\cosh (2 z / \xi)}{4 \cosh ^{3}(z / \xi)}
$$

where $n_{0}$ is the spatial concentration of impurities. By extending the integration to infinity, we have taken into account $\xi \ll w$. Performing the average and calculating the Fano factor, we find 61]

$$
F=3 / 4
$$

which is markedly different form the usual double-barrier suppression $F=1 / 2$.

The model can be generalized 15 to include Coulomb correlations 63. Imagine that each resonant center has two degenerate electron states available for tunneling, corresponding to two different spin states. However, if one of the states is filled, the other one is shifted up by the Coulomb energy $U$. We assume that the Coulomb energy is very large, so that once one electron has tunneled, the tunneling of the second one is suppressed. Then the effective tunneling rate through the "left" barrier is $2 \Gamma_{L}$ (we assume that voltage is applied from left to the right), and instead of Eqs. (76) and (77) we write for the current 63. and shot noise power per spin 61]

$$
I=\frac{e N_{V}}{\hbar} \frac{\Gamma_{L} \Gamma_{R}}{2 \Gamma_{L}+\Gamma_{R}}, \quad S=\frac{2 e^{2} N_{V}}{\hbar} \frac{\Gamma_{L} \Gamma_{R}\left(4 \Gamma_{L}^{2}+\Gamma_{R}^{2}\right)}{\left(2 \Gamma_{L}+\Gamma_{R}\right)^{3}}
$$

Averaging over impurity configurations and calculating the Fano factor, we find again $F=3 / 461$.

\footnotetext{
${ }^{15}$ This example concerns interacting systems, and is included in this Section only as an exception.
}

Experiments. The simplest experimental system one can imagine which should exhibit the features of a twobarrier structure is just a one-dimensional channel constrained by two potential barriers. If the barriers are close to each other, the region between the two barriers can be considered as a zero-dimensional system and is called a quantum dot. In addition, one usually places one more electrode (gate), which couples only capacitively to the dot. Roughly speaking, the voltage applied to the gate shifts all electron levels in the dot with respect to the chemical potential of the reservoirs, and may tune them to the resonance position. However, typically quantum dots are so small that Coulomb interaction effects (Coulomb blockade) become important, and the theoretical picture described above is no longer valid. If the space between the barriers is large and one-dimensional (one channel), interaction effects are also important, and a Luttinger liquid state is formed. For a more extensive discussion of noise in interacting systems, the reader is addressed to Section VII.

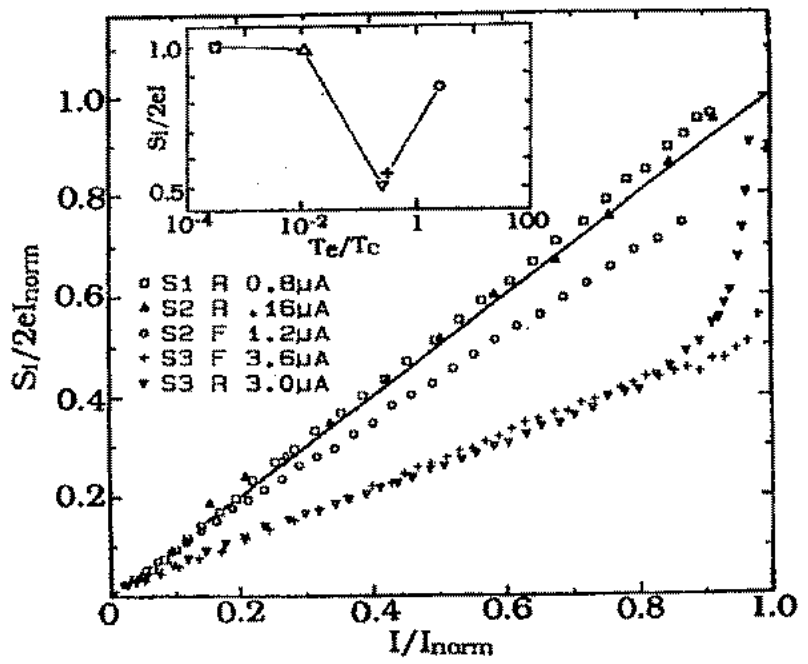

FIG. 9. The Fano factor observed experimentally by Li et al 64. as a function of current for three quantum wells, which differ by their asymmetry. The solid line represents the Poisson shot noise value.

Quantum wells, however, are macroscopic objects, and hence are less sensitive to interactions. Thus, experiments carried out on quantum wells may probe the noninteracting theory of noise suppression in a double-barrier system. Sub-Poissonian shot noise suppression in quantum wells was observed by Li et al 64 even before a theory of this suppression was available. Li et al noted that the suppression is maximal for symmetric barriers, and is insignificant for very asymmetric structures (Fig. 9). This suppression was later observed by van de Roer et al 65, Ciambrone et al 66], Liu et al 67, and Przadka et al 68. Liu et al compared their experimental data with the results of numerical simulations attempting to take into account specific features of their sample, and 
found that theory and experiment are in a reasonable agreement. Yau et al [69] observed shot noise suppression in double quantum wells (triple barrier structures). We should note, however, that in all experimental data available, the Fano factor depends considerably on the applied voltage in the whole range of voltages. Apparently, this happens because already relatively low voltages drive the system out of the linear regime. To the best of our knowledge, this issue has not been addressed systematically, although some results, especially concerning the negative differential resistance range, exist. They are summarized in Section $\mathrm{V}$.

\section{Metallic diffusive wires}

1/3-suppression. We consider now transport in multi-channel diffusive wires in the metallic regime. This means that, on one hand, the length of the wire $L$ is much longer than the mean free path $l$ due to disorder. On the other hand, in a quasi-one-dimensional geometry all electron states are localized in the presence of arbitrarily weak disorder; the localization length equals $L_{\xi}=N_{\perp} l$, where $N_{\perp}$ is the number of transverse channels 1 . Thus for a wire to be metallic we must have $L \ll L_{\xi}$ (which of course implies $N_{\perp} \gg 1$ ). As everywhere so far, we ignore inelastic processes.

Comparison between the Drude-Sommerfeld formula for conductance $\mathrm{T}^{\top 7}$,

$$
G=\frac{2}{\pi} \frac{e^{2} n \tau}{m} \frac{w}{L}
$$

( $n$ is the electron concentration, and $\tau=l / v_{F}$ is the momentum relaxation time), and the Landauer formula (40) yields the expression for the average transmission coefficient,

$$
\langle T\rangle=\frac{l}{L}
$$

In the diffusive regime we have $\langle T\rangle \ll 1$.

A naive point of view would be to assume that all the transmission coefficients of the wire are of the order of the average transmission eigenvalue $\langle T\rangle$ and thus, that all transmission probabilities are small. From our previous consideration it would then follow that the Fano factor is very close to one: a metallic diffusive wire would exhibit full Poissonian shot noise. On the other hand, it is well

\footnotetext{
${ }^{16}$ We use below two-dimensional notations: for a strip of width $w$ the number of transverse channels is equal to $N_{\perp}=$ $p_{F} w / \pi \hbar$. All results expressed through $N_{\perp}$ remain valid also for a three-dimensional (wire) geometry.

${ }^{17}$ The factor $2 / \pi$ which might look unusual to some readers only reflects a different definition of the mean free path, and is not essential for any results which we describe below.
}

known that a macroscopic metallic conductor exhibits no shot noise. Using this information as a guide one might equally naively expect that a mesoscopic metallic diffusive conductor also exhibits no shot noise.

In fact, these naive assumptions are incorrect. In particular, the fact that the transmission eigenvalues of a metallic conductor are not all small has long been recognized 18: In the metallic regime, for any energy open channels (with $T \sim 1$ ) coexist with closed ones $(T \ll 1$ ). The distribution function of transmission coefficients has in fact a bimodal form. This bimodal distribution leads to sub-Poissonian shot noise. Quantitatively, this situation can be described by random matrix theory of onedimensional transport. It implies 73 that the channeldependent inverse localization lengths $\zeta_{n}$, related to the transmission coefficients by $T_{n}=\cosh ^{-2}\left(L / \zeta_{n}\right)$, are uniformly distributed between 0 and $l^{-1}$. This statement can be transformed into the following expression for the distribution function of transmission coefficients,

$$
\begin{array}{r}
P(T)=\frac{l}{2 L} \frac{1}{T \sqrt{1-T}}, \quad T_{\min }<T<1, \\
T_{\min }=4 \exp (-2 L / l),
\end{array}
$$

and $P(T)=0$ otherwise. As discussed, it has a bimodal form: almost open and almost close channels are preferred. The dependence $P(T)$ is illustrated in Fig. 10 .

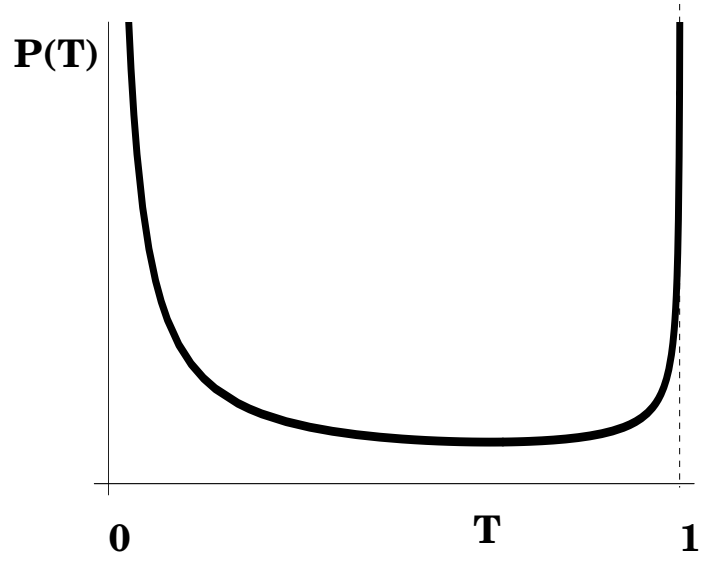

FIG. 10. Distribution function of transmission coefficients (87) for $L / l=10$.

The distribution function $P(T)$ must be used now to average expressions (40) and (57) over impurity configurations. Direct calculation confirms Eq. (86), and, thus, the distribution function (87) yields the DrudeSommerfeld formula (85) for the average conductance. Furthermore, we obtain

\footnotetext{
${ }^{18}$ This statement has a long history, and we only cite two early papers on the subject by Dorokhov 70 and Imry 71 . For a modern discussion we refer to Ref. 72 .
} 


$$
\langle T(1-T)\rangle=\frac{l}{3 L},
$$

which implies that the zero-temperature shot noise power is

$$
S=\frac{e^{3}|V|}{3 \pi \hbar} \frac{N_{\perp} l}{L}=\frac{1}{3} S_{P}
$$

The shot noise suppression factor for metallic diffusive wires is equal to $F=1 / 3$. The remarkable feature is that this result is universal: As long as the geometry of the wire is quasi-one-dimensional and $l \ll L \ll L_{\xi}$ (metallic diffusive regime), the Fano factor does not depend on the degree of disorder ${ }^{T 9}$, the number of transverse channels, and any other individual features of the sample. This result was first obtained by Beenakker and one of the authors [74] using the approach described above. Independently, Nagaev [75] derived the same suppression factor $1 / 3$ by using a classical theory based on a Boltzmann equation with Langevin sources. This theory and subsequent developments are described in Section VI.

Later on, the $1 / 3$ suppression of shot noise became a subject of a number of microscopic derivations. Altshuler, Levitov, and Yakovets [76] recovered the Fano factor $1 / 3$ by direct microscopic calculation using the Green's function technique. Nazarov [77] proved that the distribution (87) holds for an arbitrary (not necessarily quasi-one-dimensional) geometry; thus, the $1 / 3$ suppression is "super-universal". He used a slightly different technique, expressing scattering matrices through Green's functions and then performing disorder averages. The same technique, in more elaborated form, was used in Ref. [78], which also obtains the $1 / 3$-suppression.

It is clear that the quantum-mechanical theories of Refs. 74, 76 78 are equivalent for the quasi-onedimensional geometry, since they deal with disorder averages basically in the same way. On the other hand, their equivalence to the classical consideration of Ref. [75] is less obvious.

Experimentally, shot noise in metallic diffusive wires was investigated by Liefrink et al 79], who observed that it is suppressed with respect to the Poisson value. The suppression factor in this experiment lies between 0.2 and 0.4 (depending on gate voltage). More precise experiments were performed by Steinbach, Martinis, and Devoret [80] who analyzed silver wires of different length. In the shortest wires examined they found a shot noise slightly larger than $1 / 3$ and explained this larger value as due to electron-electron interaction 20 . A very accurate

\footnotetext{
${ }^{19}$ It seems that the question whether the Fano factor depends on the type of disorder has never been addressed. In all cases disorder is assumed to be Gaussian white noise, i.e. $\left\langle U(\boldsymbol{r}) U\left(\boldsymbol{r}^{\prime}\right)\right\rangle \propto \delta\left(\boldsymbol{r}-\boldsymbol{r}^{\prime}\right)$.

${ }^{20}$ In long wires, interaction effects play a role; this is addressed in Section VI.
}

measurement of the $1 / 3$ noise suppression was performed by Henny et al [81]. Special care was taken to avoid electron heating effects by attaching very large reservoirs to the wire. Their results are displayed in Fig. 11.

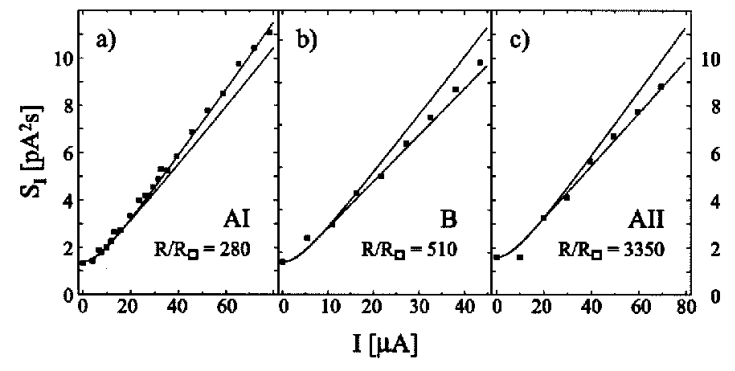

FIG. 11. Shot noise measurements by Henny et al 81 on three different samples. The lower solid line is 1/3-suppression, the upper line is the hot-electron result $F=\sqrt{3} / 4$ (see Section $\mathrm{VI}$ ). The samples (b) and (c) are short, and clearly display 1/3-suppression. The sample (a) is longer (has lower resistance), and the shot noise deviates from the non-interacting suppression value due to inelastic processes.

Localized regime. In quasi-one-dimensional wires with length $L \gg L_{\xi}$ the transmission coefficients are "crystallized" around exponentially small values [72]. This leads to a conductance and a shot noise power which decay exponentially with the length $L$. Shot noise is not suppressed with respect to the Poissonian value: $F=1$.

The shot noise in the one-dimensional case was analyzed by Melnikov [56], who obtained different suppression factors for various models of disorder in a onechannel wires. In particular, the model of Gaussian deltacorrelated one-dimensional disorder leads to the suppression factor $3 / 4$.

Weak localization and mesoscopic fluctuations. In the metallic regime, quantum interference effects due to disorder, which eventually drive the system into the localized regime, manifest themselves in the form of weak localization corrections. For the shot noise, the weak localization correction was studied by de Jong and Beenakker 82], and later by Macêdo [83], Macêdo and Chalker 84, and Macêdo [85]. They found

$$
S=\frac{e^{3}|V|}{\pi \hbar}\left[\frac{N_{\perp} l}{3 L}-\frac{1}{45}\right] .
$$

Comparison with a similar expression for the conductance,

$$
G=\frac{e^{2}}{2 \pi \hbar}\left[\frac{N_{\perp} l}{L}-\frac{1}{3}\right],
$$

yields the Fano factor

$$
F=\frac{1}{3}+\frac{4}{45} \frac{L}{N_{\perp} l} .
$$

The second terms represent weak localization corrections $\left(L \ll N_{\perp} l\right)$. These expressions are valid for the case 
of preserved time-reversal symmetry (orthogonal symmetry). In the case of broken time-reversal symmetry (unitary symmetry; technically, this means that a weak magnetic field is applied) weak localization corrections are absent and the Fano factor stays at $1 / 3$. Thus, we see that weak localization effects suppress noise, but enhance the Fano factor, in agreement with the general expectation that it lies above $1 / 3$ in the localized regime. The crossover from the metallic to the localized regime for shot noise has not been investigated.

De Jong and Beenakker 82, Macêdo 83, and Macêdo and Chalker 84 studied also mesoscopic fluctuations of shot noise 21 , which are an analog of the universal conductance fluctuations. For the root mean square of the shot noise power, they found

$$
\text { r.m.s. } S=\frac{e^{3}|V|}{\pi \hbar} \sqrt{\frac{46}{2835 \beta}},
$$

where the parameter $\beta$ equals 1 and 2 for the orthogonal and unitary symmetry, respectively. These fluctuations are independent of the number of transverse channels, length of the wire, or degree of disorder, and may be called 82 "universal noise fluctuations".

The picture which emerges is, therefore, that like the conductance, the ensemble averaged shot noise is a classical quantity. Quantum effects in the shot noise manifest themselves only if we include weak localization effects or if we ask about fluctuations away from the average. With these results it is thus no longer surprising that the $1 / 3$ noise suppression factor derived quantum mechanically and from a classical Boltzmann equation for the fluctuating distribution are in fact the same. The same picture holds of course not only for metallic diffusive wires but whenever we ensemble average. We have already discussed this for the resonant double barrier and below will learn this very same lesson again for chaotic cavities.

Chiral symmetry. Mudry, Brouwer, and Furusaki [86,87 studied the transport properties of disordered wires with chiral symmetry (i.e. when the system consists of two or several sublattices, and only transitions between different sublattices are allowed). Examples of these models include tight-binding hopping models with disorder or the random magnetic flux problem. Chiral models exhibit properties usually different from those of standard disordered wires 22 , for instance, the conductance at the band center scales not exponentially with the length of the wire $L$, but rather as a power law [88. Ref. 87], however, finds that in the diffusive regime $\left(l \ll L \ll N_{\perp} l\right)$ the Fano factor equals precisely $1 / 3$, like for ordinary symmetry. The only feature which appears due to the chiral symmetry is the absence of weak

\footnotetext{
${ }^{21}$ This requires the knowledge of the joint distribution function of two transmission eigenvalues.

${ }^{22}$ Extensive list of references is provided by Ref. [86.
}

localization corrections in the zero order in $N_{\perp}$. Weak localization corrections, both for conductance and shot noise, scale as $L /\left(l N_{\perp}\right)$ and discriminate between chiral unitary and chiral orthogonal symmetries.

Transition to the ballistic regime. In the zerotemperature limit, a perfect wire does not exhibit shot noise. For this reason, one should anticipate that in the ballistic regime, $l>L$, shot noise is suppressed below $1 / 3$. The crossover between metallic and ballistic regimes in disordered wires was studied by de Jong and Beenakker 82 (see their Eq. (A10)), who found for the noise suppression factor

$$
F=\frac{1}{3}\left(1-\frac{1}{(1+L / l)^{3}}\right) .
$$

It, indeed, interpolates between $F=1 / 3$ for $l \ll L$ and $F=0$ for $L \gg l$. Later, they [89,90 illustrated Eq. (91) by using the classical (Boltzmann-Langevin) approach for single-channel wires ${ }^{23}$. Liu, Eastman, and Yamamoto [91] performed Monte Carlo simulations of shot noise for the same situation, and found agreement with Eq. (91).

Nazarov [77 and, independently, Beenakker and Melsen 92 addressed the metallic - ballistic crossover in a disordered quantum point contact, i.e. a constriction between two quasi-one-dimensional metallic diffusive conductors (of identical width). The diffusive conductors have a mean free path $l$, a combined length $L$ and a total resistance $R_{N}$. The constriction has a resistance $R_{T}=\left(h / e^{2}\right) N_{0}$ in the presence of $N_{0}$ open channels. For this system the Fano factor is a function of the ratio $\gamma=N_{0} L /(l N)=R_{N} / R_{T}$, and is given by

$$
F=\frac{1}{3}\left(1-\frac{1}{(1+\gamma)^{3}}\right) \text {. }
$$

Eq.(92) describes a crossover from $F=1 / 3$ (the metallic regime) to $F=1$ (classical point contact between metallic diffusive banks) and actually follows 92 from Eq. (91).

Disordered interfaces. Schep and Bauer 93 considered transport through disordered interfaces, modeled as a configuration of short-ranged scatterers randomly distributed in the plane perpendicular to the direction of transport. In the limit $g \ll N_{\perp}$, with $g$ and $N_{\perp}$ being the dimensionless conductance and the number of transverse channels, respectively, they found the following distribution function of transmission coefficients,

$$
\begin{aligned}
P(T)= & \frac{g}{\pi N_{\perp}} \frac{1}{T^{3 / 2} \sqrt{1-T}}, \\
& {\left[1+\left(\frac{\pi N_{\perp}}{2 g}\right)^{2}\right]^{-2}<T<1, }
\end{aligned}
$$

\footnotetext{
${ }^{23}$ This must be considered as a toy model, since the classical theory ignores localization effects. Single-channel wires are in reality either ballistic or localized, but never metallic.
} 
and zero otherwise. Eq. (93) accidentally has the same form as the distribution function of transmission coefficients for the symmetric opaque double-barrier structure. The noise suppression factor for this system equals $1 / 2$, e.g. the suppression is weaker than for metallic diffusive wires.

\section{Chaotic cavities}

1/4-suppression. Chaotic cavities are quantum systems which in the classical limit would exhibit chaotic electron motion. We consider ballistic chaotic systems without any disorder inside the cavity; the chaotic nature of classical motion is a consequence of the shape of the cavity or due to surface disorder. The results presented below are averages over ensembles of cavities. The ensemble can consist of a collection of cavities with slightly different shape or a variation in the surface disorder, or it can consist of cavities investigated at slightly different energies. Experimentally, chaotic cavities are usually realized as quantum dots, formed in the 2D electron gas by back-gates. They may be open or almost closed; we discuss first the case of open chaotic quantum dots, shown in Fig. 12a. We neglect charging effects 24. One more standard assumption, which we use here, is that there is no direct transmission: Electrons incident from one lead cannot enter another lead without being reflected from the surface of the cavity (like Fig. 12a).

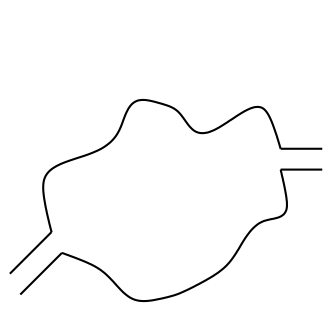

a)

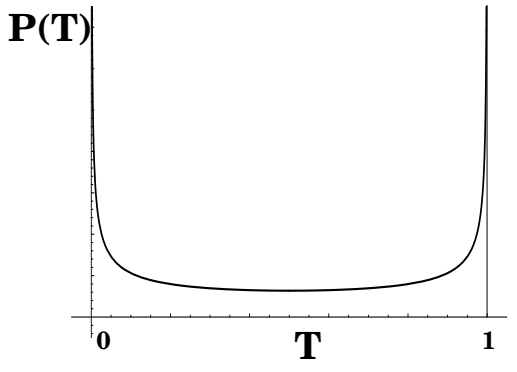

b)
FIG. 12. (a) An example of a chaotic cavity. (b) Distribution function of transmission eigenvalues (94).

The description of transport properties of open chaotic cavities based on the random matrix theory was proposed independently by Baranger and Mello [96] and Jalabert, Pichard, and Beenakker 97]. They assumed that the scattering matrix of the chaotic cavity is a member of

\footnotetext{
${ }^{24}$ In the case when the cavity is open, i.e. connected by ideal leads to the electron reservoirs, charging effects may still play a role. This effect, called mesoscopic charge quantization 94, was recently shown to affect very weakly the conductance of open chaotic cavities 95. Results for shot noise are currently unavailable.
}

Dyson's circular ensemble of random matrices, uniformly distributed over the unitary group. For the cavity where both left and right leads support the same number of transverse channels $N_{\perp} \gg 1$, this conjecture implies the following distribution function of transmission eigenvalues,

$$
P(T)=\frac{1}{\pi \sqrt{T(1-T)}},
$$

shown in Fig. 12 b. As a consequence of the assumption underlying random matrix theory, this distribution is universal: It does not depend on any features of the system. Taking into account that $\langle T\rangle=1 / 2$ and $\langle T(1-T)\rangle=1 / 8$, we obtain for conductance,

$$
G=\frac{e^{2} N_{\perp}}{4 \pi \hbar},
$$

and for the zero-temperature shot noise,

$$
S=\frac{e^{3}|V| N_{\perp}}{8 \pi \hbar}=\frac{1}{4} S_{P}
$$

The Fano factor equals $F=1 / 4$, and is, of course, also universal. For the one-channel case, the whole distribution function of shot noise may be found analytically [98],

$$
\begin{aligned}
& P(S) \\
= & \begin{array}{cc}
\frac{\sqrt{1+\sqrt{1-4 \eta}}+\sqrt{1-\sqrt{1-4 \eta}}}{\sqrt{16 \eta(1-4 \eta)}}, & \text { orthogonal symmetry } \\
\frac{2}{\sqrt{1-4 \eta}}, & \text { unitary symmetry }
\end{array}
\end{aligned}
$$

where we defined $\eta=\pi \hbar S /\left(e^{3}|V|\right)$.

In the general case when the numbers of transverse channels in the left $N_{L}$ and right $N_{R}$ lead are not equal, but still $N_{L} \gg 1$ and $N_{R} \gg 1$, the distribution function of transmission eigenvalues has been calculated by Nazarov 100]. Using this result, a calculation of the shot noise gives for the Fano factor 10072

$$
F=\frac{N_{L} N_{R}}{\left(N_{L}+N_{R}\right)^{2}}
$$

This suppression factor has $1 / 4$ as its maximal value for the symmetric case $N_{L}=N_{R}$, and shot noise is suppressed down to zero in the very asymmetric case $N_{L} \ll N_{R}$ or $N_{L} \gg N_{R}$. Indeed, for $N_{L} \ll N_{R}$ the transport properties are determined by the less transparent (left) contact; however, since the contact is still ideal, the noise in this situation is totally suppressed.

Eq. (96) results from an ensemble average, and, as we discussed, must be a classical result. Indeed, it has been derived [99] by purely classical means, see Section VI.

Crossover to double-barrier behavior. We assume now that the cavity is separated from the leads by tunnel barriers. Brouwer and Beenakker [101] were able to calculate the distribution function of transmission coefficients in this system for the symmetric case, when the 
number of channels supported by the left and the right lead are equal, $N_{L}=N_{R}=N$, and the transmission coefficients $\tilde{T}_{i}$ in each channel $i$ are same for the left and the right barriers. Assuming in addition $N \tilde{T}_{i} \gg 1$ for all channels, they found

$$
P(T)=\frac{1}{N} \sum_{i=1}^{N} \frac{\tilde{T}_{i}\left(2-\tilde{T}_{i}\right)}{\pi\left(\tilde{T}_{i}^{2}-4 \tilde{T}_{i} T+4 T\right) \sqrt{T(1-T)}} .
$$

Calculating the averages

$$
\langle T\rangle=\frac{1}{2 N} \sum_{i} \tilde{T}_{i}
$$

and

$$
\langle T(1-T)\rangle=\frac{1}{8 N} \sum_{i} \tilde{T}_{i}\left(2-\tilde{T}_{i}\right)
$$

we find the Fano factor,

$$
F=\frac{\langle T(1-T)\rangle}{\langle T\rangle}=\frac{1}{4} \frac{\sum_{i} \tilde{T}_{i}\left(2-\tilde{T}_{i}\right)}{\sum_{i} \tilde{T}_{i}} .
$$

In particular, if all the transmission probabilities $\tilde{T}_{i}$ are the same and equal to $\tilde{T}$, we obtain $F=(2-\tilde{T}) / 4$. This expression reproduces the limiting cases $F=1 / 4$ for $\tilde{T}=1$ (no barriers - open quantum cavity) and $F=$ $1 / 2$ for $\tilde{T} \rightarrow 0$ (double-barrier suppression in symmetric system). Thus, Eq. (98) describes crossover between the behavior characteristic for an open cavity and the situation when the barriers are so high that the dynamics inside the cavity does not play any role.

\section{Edge channels in the quantum Hall effect regime}

Now we turn to the description of effects which are inherently multi-terminal. The calculation of the scattering matrix is in general a difficult problem. However, in some special situations the scattering matrix can be deduced immediately even for multi-terminal conductors.

We consider a four-terminal conductor (Fig. 13) made by patterning a two-dimensional electron gas. The conductor is brought into the quantum Hall regime by a strong transverse magnetic field. In a region with integer filling of Landau levels the only extended states at the Fermi energy which connect contacts 102 are edge states 25 , the quantum mechanical equivalent of classical

\footnotetext{
${ }^{25}$ We do not give a microscopic description of edge states. Coulomb effects in the integer quantum Hall effect regime lead to a spatial decomposition into compressible and incompressible regions. Edge channels in the fractional quantum Hall effect regime will be discussed in Section VII.
}

skipping orbits. Since the net current at a contact is determined by the states near the Fermi surface, transport in such a system can be described by considering the edge states. Note that this fact makes no statement on the spatial distribution of the current density. In particular a description based on edge states does not mean that the current density vanishes away from the edges. This point which has caused considerable confusion and generated a number of publications is well understood, and we refer the reader here only to one particularly perceptive discussion 103. Edge states are uni-directional; if the sample is wide enough, backscattering from one edge state to another one is suppressed [102. In the plateau regime of the integer quantum Hall effect, the number of edge channels is equal to the number of filled Landau levels. For the discussion given here, we assume, for simplicity, that we have only one edge state. In a quantum Hall conductor wide enough so that there is no backscattering, there is no shot noise 18]. Hence we introduce a constriction (Fig. 13) and allow scattering between different edge states at the constriction 18 : the probability of scattering from contact 4 to the contact 3 is $T$, while that from 4 to 1 is $1-T$. In the following, we will focus on the situation when the chemical potentials of all the four reservoirs are arranged so that $\mu_{2}=\mu_{3}=\mu$, $\mu_{1}=\mu_{4}=\mu+e V$.

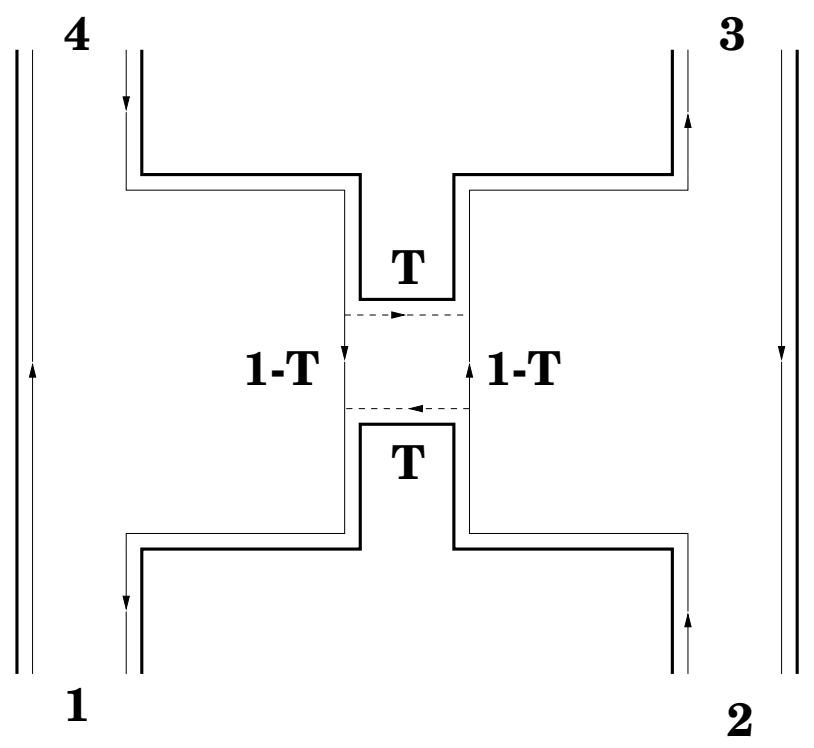

FIG. 13. Four-probe quantum Hall conductor. Solid lines indicate edge channels; dashed lines show the additional scattering probability $T$ through the quantum point contact.

The scattering matrix of this system has the form

$$
s=\left(\begin{array}{cccc}
0 & s_{12} & 0 & s_{14} \\
0 & 0 & s_{23} & 0 \\
0 & s_{32} & 0 & s_{34} \\
s_{41} & 0 & 0 & 0
\end{array}\right)
$$

Here the elements $s_{14}=r, s_{32}=r^{\prime}, s_{12}=t, s_{34}=t^{\prime}$ form the $2 \times 2$ scattering matrix at the constriction: $|t|^{2}=$ 
$\left|t^{\prime}\right|^{2}=T,|r|^{2}=\left|r^{\prime}\right|^{2}=1-T, r^{*} t^{\prime}+t^{*} r^{\prime}=r^{*} t+r^{\prime} t^{\prime *}=0$. The two remaining elements, $s_{41}=\exp \left(i \theta_{1}\right)$ and $s_{23}=$ $\exp \left(i \theta_{2}\right)$ describe propagation along the edges without scattering. It is straightforward to check that the matrix (99) is unitary.

We consider first the shot noise at zero temperature [18,9]. Using the general Eq. (55), we see immediately that the only non-zero components of the shot noise power tensor are $S_{11}=S_{33}=-S_{13}=-S_{31}$, with

$$
S_{31}=-\frac{e^{3}|V|}{\pi \hbar} T(1-T) .
$$

Indeed, if there is no scattering between the edge states, there is no shot noise in the system. The same is true for the case when this scattering is too strong: all the current from 2 flows to 1 , and from 4 to 3 .

For finite temperature all components become non-zero (except for $S_{24}$ ) and can be found [9] from Eq. (52). We only give the result for $S_{13}=S_{31}$,

$$
S_{13}=-\frac{e^{2}}{\pi \hbar} \int d E T(1-T)\left(f_{1}-f_{3}\right)^{2},
$$

where $f_{1}$ and $f_{3}$ are Fermi distribution functions in the reservoirs 1 and 3, respectively. This result is remarkable since it vanishes for $T=1$ at any temperature: The correlation function (101) is always "shot-noise-like".

One more necessary remark is that all the shot noise components in this example are actually expressed only through absolute values of the scattering matrix elements: Phases are not important for noise in this simple edge channel problem.

Early experiments on noise in quantum Hall systems were oriented to other sources of noise (see e.g. Refs. 104,105), and are not discussed here. Shot noise in the quantum Hall regime was studied by Washburn et al 106. who measured voltage fluctuations in a six-terminal geometry with a constriction, which, in principle, allows for direct comparison with the above theory. They obtained results in two magnetic fields, corresponding to the filling factors $\nu=1$ and $\nu=4$. Although their results were dominated by $1 / f$-noise, Washburn et al were able to find that shot noise is very much reduced below the Poisson value, and the order of magnitude corresponds to theoretical results.

\section{Hanbury Brown - Twiss Effects with edge channels}

The conductor of Fig. 13 is an electrical analog of the scattering of photons at a half-silvered mirror (see Fig. 11). Like in the table top experiment of Hanbury Brown and Twiss [7], there is the possibility of two sources which send particles to an object (here the quantum point contact) permitting scattering into transmitted and reflected channels which can be detected separately. Bose statistical effects have been exploited by Hanbury Brown and
Twiss 7 to measure the diameter of stars. The electrical geometry of Fig. 13 was implemented by Henny et al [12], and the power spectrum of the current correlation between contact 1 (reflected current) and contact 3 (transmitted current) was measured in a situation where current is incident from contact 4 only. Contact 2 was closed such that effectively only a three-terminal structure resulted. For the edge-channel situation considered here, in the zero-temperature limit, this does not affect the correlation between transmitted and reflected current. (At finite temperature, the presence of the fourth contact would even be advantageous, as it avoids, as described above, the "contamination" due to thermal noise of the correlation function of reflected and transmitted currents, see Eq. (101)). The experiment by Henny et al finds good agreement with the predictions of Ref. [18], i.e. Eq. (100). With experimental accuracy the current correlation $S_{31}$ is negative and equal in magnitude to the mean square current fluctuations $S_{11}=S_{33}$ in the transmitted and reflected beam. The experiment finds thus complete anti-correlation. This outcome is related to the Fermi statistics only indirectly: If the incident carrier stream is noiseless, current conservation alone leads to Eq. (100). As pointed out by Henny et al 12], the experiment is in essence a demonstration that in Fermi systems the incident carrier stream is noiseless. The pioneering character of the experiment by Henny et al $[12$ and an experiment by Oliver et al [107] which we discuss below lies in the demonstration of the possibility of measuring current-current correlation in electrical conductors [108]. Henny et al 12 measured not only the shot noise but used the four-terminal geometry of Fig. 13 to provide an elegant and interesting demonstration of the fluctuationdissipation relation, Eq. (54).

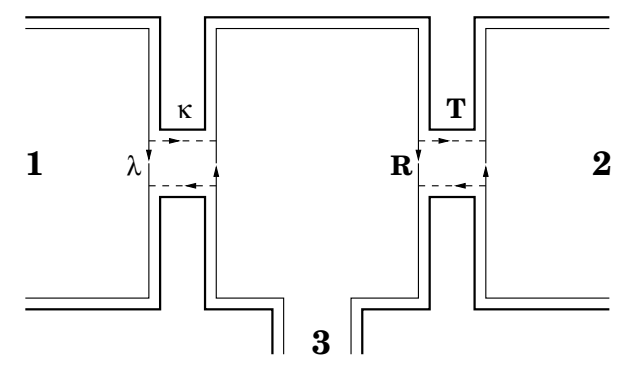

FIG. 14. Three-probe geometry illustrating the experiment by Henny et al [12].

Now it is interesting to ask, what happens if this complete population of the available states is destroyed (the incident carrier stream is not noiseless any more). This can be achieved by inserting an additional quantum point contact in the path of the incident carrier beam (see Fig. 14). We denote the transmission and reflection probability of this first quantum point contact by $\kappa$ and $\lambda=1-\kappa$, and the transmission and reflection probability of the second quantum point contact by $T$ and $R$ as above. The scattering matrix of this system has the form 


$$
s=\left(\begin{array}{ccc}
-i \lambda^{1 / 2} & 0 & \kappa^{1 / 2} \\
(\kappa T)^{1 / 2} & -i R^{1 / 2} & -i(\lambda T)^{1 / 2} \\
-i(\kappa R)^{1 / 2} & T^{1 / 2} & -(\lambda R)^{1 / 2}
\end{array}\right) .
$$

The phases in this experiment play no role and here have been chosen to ensure the unitarity of the scattering matrix. In the zero-temperature limit with a voltage difference $V$ between contact 1 and contacts 2 and 3 (which are at the same potential) the noise power spectra are

$$
S=\frac{e^{2}|V|}{\pi \hbar}\left(\begin{array}{ccc}
\kappa \lambda & -\kappa \lambda T & -\kappa \lambda R \\
-\kappa \lambda T & \kappa T(1-\kappa T) & -\kappa^{2} T R \\
-\kappa \lambda R & -\kappa^{2} T R & \kappa R(1-\kappa R)
\end{array}\right)
$$

The correlation function between transmitted and reflected beams $S_{23}=S_{32}=-\kappa^{2} R T$ is proportional to the square of the transmission probability in the first quantum point contact. For $\kappa=1$ the incident beam is completely filled, and the results of Henny et al 12] are recovered. In the opposite limit, as $\kappa$ tends to zero, almost all states in the incident carrier stream are empty, and the anti-correlation between transmitted and reflected beams also tends to zero.

\section{Three-terminal structures in zero magnetic field}

A current-current correlation was also measured in an experiment by Oliver et al [107] in a three-probe structure in zero magnetic field. This experiment follows more closely the suggestion of Martin and Landauer [21] to consider the current-current correlations in a Ystructure. Ref. [21], like Ref. [18], analyzes the noise power spectrum in the zero frequency limit. Early experiments on a three-probe structure by Kurdak et al 109 were dominated by $1 / f$-noise and did not show any effect.

Here the following remark is appropriate. Strictly speaking, the Hanbury Brown - Twiss (HBT) effect is a coincidence measurement. In the optical experiment the intensity fluctuation $d I_{\alpha}(t)$ is measured and correlated with the intensity fluctuation $d I_{\beta}(t+\tau)$, where $\tau$ is a short time smaller than the response time $\tilde{\tau}$ of the detector. The coincidence rate $C_{\alpha \beta}$ is thus

$$
C_{\alpha \beta}=(1 / 2 \tilde{\tau}) \int_{0}^{\tilde{\tau}} d \tau\left\langle d \hat{I}_{\alpha}(t) d \hat{I}_{\beta}(t+\tau)+d \hat{I}_{\beta}(t+\tau) d \hat{I}_{\alpha}(t)\right\rangle
$$

The coincidence rate is related to the frequency dependent noise power spectrum by

$$
C_{\alpha \beta}=(1 / \tilde{\tau}) \int_{0}^{\tilde{\tau}} d \tau \int d \omega e^{i \omega \tau} S_{\alpha \beta}(\omega)
$$

In Section III, we discuss the frequency dependence of the noise power spectrum in more detail. Typically its lowest characteristic frequencies are given by $R C$-times.
In principle, such a measurement should, therefore, be able to give information on the frequency dependence of the noise power spectrum. In the experiment of Oliver et al the resolution time $\tilde{\tau}$ is probably long compared to such intrinsic time scales, and thus the experiment is effectively determined by the white noise limit of the power spectrum.

Let us now briefly consider a Y-shaped conductor 21 and discuss its correlations in the white noise limit. We assume that the same voltage $V$ is applied between the terminals 1 and 2, and 1 and 3: $\mu_{1}=\mu_{2}+e V=\mu_{3}+e V$. For zero temperature, the general formula (55) yields the following expression for the cross-correlations of currents in leads 2 and 3 ,

$$
S_{23}=-\frac{e^{3}|V|}{\pi \hbar} \operatorname{Tr}\left[s_{21}^{\dagger} s_{21} s_{31}^{\dagger} s_{31}\right],
$$

which is negative in accordance with the general considerations. Note the formal similarity of this result to the shot-noise formula in the two terminal geometry given by Eq. (56). In the single channel limit, if we assume that there is no reflection back into contact 1, Eq. (106) becomes $S_{23}=-\left(e^{3}|V| / \pi \hbar\right) T(1-T)$, where $T$ is the transmission probability from 1 to 2 . This simple result underlines (the formal) equivalence of scattering at a QPC with separation of transmitted and reflected streams and scattering at a reflectionless $\mathrm{Y}$-structure. The experiments by Oliver et al [107] confirm these theoretical predictions.

The experiments by Henny et al [12] and Oliver et al 107 test the partitioning of a current stream. If the incident carrier stream is noiseless, the resulting current correlation is negative already due to current conservation alone. Therefore, experiments are desirable, which test electron statistical effects (and the sign of correlations) in situations where current conservation plays a much less stringent role.

\section{Exchange Hanbury Brown - Twiss effects}
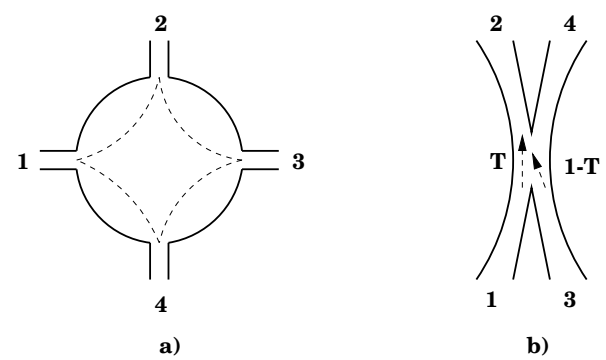

FIG. 15. Four-terminal conductors for Hanbury Brown Twiss exchange effects (a), quantum point contact geometry (b). Terminals are numbered by digits. The dashed line in (a) indicates a phase-sensitive trajectory which contributes to exchange terms in Eq. (107). Arrows in (b) indicate non-zero transmission probabilities. 
Another HBT experiment was proposed in Ref. 19] (see also Ref. [9]). It is based on the comparison of the noise generated in the presence of two incident currents with the noise generated by one source only. We first present a general discussion and later consider a number of applications. Consider the four-terminal structure of Fig. 15a. We will be interested in cross-correlations of currents at the contacts 2 and 4 ,

$$
S \equiv-S_{24}
$$

The quantity $S$ defined in this way is always positive. Now we discuss three different ways of applying voltage. The first one (to be referred to as experiment A) is to apply a voltage to the reservoir $1, \mu_{1}-e V=\mu_{2}=\mu_{3}=$ $\mu_{4}$. In the next one (experiment B) the voltage is applied to $3, \mu_{1}=\mu_{2}=\mu_{3}-e V=\mu_{4}$. Finally, in the experiment $\mathrm{C}$ the identical ${ }^{26}$ voltages are applied to 1 and $3, \mu_{1}-$ $\mathrm{eV}=\mu_{2}=\mu_{3}-\mathrm{eV}=\mu_{4}$. Results for zero temperature are readily derived from Eq. (55) and read

$$
\begin{aligned}
& S_{A}=\frac{e^{3}|V|}{\pi \hbar} \Xi_{1}, \quad S_{B}=\frac{e^{3}|V|}{\pi \hbar} \Xi_{2}, \\
& S_{C}=\frac{e^{3}|V|}{\pi \hbar}\left(\Xi_{1}+\Xi_{2}+\Xi_{3}+\Xi_{4}\right),
\end{aligned}
$$

where

$$
\begin{aligned}
& \Xi_{1}=\operatorname{Tr}\left[s_{21}^{\dagger} s_{21} s_{41}^{\dagger} s_{41}\right], \\
& \Xi_{2}=\operatorname{Tr}\left[s_{23}^{\dagger} s_{23} s_{43}^{\dagger} s_{43}\right], \\
& \Xi_{3}=\operatorname{Tr}\left[s_{21}^{\dagger} s_{23} s_{43}^{\dagger} s_{41}\right] \\
& \Xi_{4}=\operatorname{Tr}\left[s_{23}^{\dagger} s_{21} s_{41}^{\dagger} s_{43}\right] .
\end{aligned}
$$

The quantities $S_{A}$ and $S_{B}$ are determined by transmission probabilities from 2 and 4 to 1 and 3, respectively, and are not especially interesting. New information is contained in $S_{C}$. In systems obeying classical statistics, the experiment $\mathrm{C}$ would be just a direct superposition of the experiments A and B, $S_{C}=S_{A}+S_{B}$. The additional terms $\Xi_{3}$ and $\Xi_{4}$ in the rhs of Eq. (107) are due to quantum (Fermi) statistics of the electrons. These terms now invoke products of scattering matrices which are in general not real valued. These terms are not products of two pairs of scattering matrices as in Eq. (56) or Eq. (106) but contain four scattering matrices in such a way that we are not able to distinguish from which of the two current carrying contacts a carrier was incident. For future convenience, we define the quantity $\Delta S=S_{C}-S_{A}-S_{B}$,

\footnotetext{
${ }^{26} \mathrm{We}$ note in passing that if different voltages are applied to 1 and 3, the correlation functions (107) imply that $S_{23}$ cannot be an analytic function of the two voltages. Thus, our four-terminal conductor is a non-linear circuit element due to exchange effects.
}

which indicates the fermionic analog of the HBT effect. One can show 9 that for finite temperatures the corresponding correction for bosons is of the same form but has the opposite sign, hence it will be called "exchange contribution". One more remarkable feature of the result (107) is that the exchange correction $\Delta S$ is phase sensitive. Indeed, it represents the contribution of trajectories indicated by the dashed line in Fig. 15a (traversed in both directions), and thus is proportional to $\exp ( \pm i \phi)$, with $\phi$ being the phase accumulated during the motion along the trajectories. For this reason, one cannot generally predict the sign of $\Delta S$ : the only restrictions are that all the quantities $S_{A}, S_{B}$, and $S_{C}$ need to be positive.

Gramespacher and one of the authors 110 112 considered a particular geometry where the leads 2 and 4 are tunneling contacts locally coupled to the sample (e.g. scanning tunneling microscope tips). In this case, the exchange contribution can directly be expressed in terms of the wave functions (scattering states)

$$
\begin{aligned}
\Delta S & =\frac{1}{\pi^{2} \hbar^{2}} \sum_{m n} \frac{1}{v_{1 m} v_{3 n}} \\
& \times \operatorname{Re}\left\{\psi_{1 m}(\boldsymbol{r}) \psi_{1 m}^{*}\left(\boldsymbol{r}^{\prime}\right) \psi_{3 n}^{*}(\boldsymbol{r}) \psi_{3 n}\left(\boldsymbol{r}^{\prime}\right)\right\},
\end{aligned}
$$

where the sum is over all transverse channels $m$ in the lead 1 and $n$ in the lead $3 ; \boldsymbol{r}$ and $\boldsymbol{r}^{\prime}$ are the points to which the contacts 2 and 4 couple, respectively, and $\psi_{\alpha k}$ is the wave function of the corresponding scattering state. Thus, the exchange contribution explicitly depends on phases of the wave functions.

We investigate now the general expression for the fourterminal phase-sensitive HBT effect (107) for various systems. Our concern will be the sign and relative magnitude of the exchange contribution $\Delta S=S_{C}-S_{A}-S_{B}$.

Disordered systems. Naively, one might assume that in a disordered medium the phase accumulated along the trajectory, indicated by the dashed line in Fig. 15a, is random. Then the phase-sensitive exchange contribution would be zero after being averaged over disorder. Thus, this view implies $S_{C}=S_{A}+S_{B}$.

A quantitative analysis of these questions was provided by the authors of this review in Ref. [78]. In this work the scattering matrices in Eq. (108) are expressed through Green's functions to which disorder averaging was applied using the diagram technique. The key result found in Ref. 78 is that the naive picture mentioned above, according to which one might expect no exchange effects after disorder averaging, is completely wrong. Exchange effects survive disorder averaging. The reason can be understood if the principal diagrams (which contain four diffusion propagators) are translated back into the language of electron trajectories. One sees then that the typical trajectory does not look like the dashed line in Fig. 15b. Instead, it looks like a collection of dashed lines shown in Fig. 16a: the electron diffuses from contact 1 to some intermediate point 5 in the bulk of the sample (eventually, the result is integrated over the coordinate of point 5 ), then it diffuses from 5 to 2 and back from 2 
to 5 precisely along the same trajectory, and so on, until it returns from 5 to 1 along the same diffusive trajectory as it started. Thus, there is no phase enclosed by the trajectory. This explains why the exchange contribution survives averaging over disorder; apparently, there is a classical contribution to the exchange correlations which requires knowledge only of Fermi statistics, but no information about phases of scattering matrices. Indeed, a classical theory of ensemble averaged exchange effects was subsequently proposed by Sukhorukov and Loss 113,114.

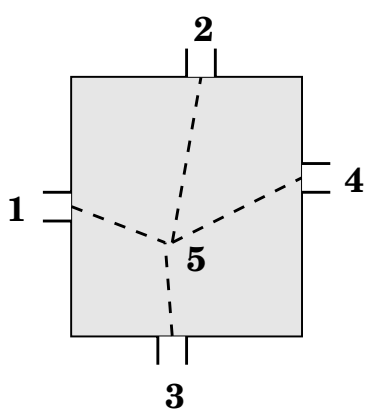

a)

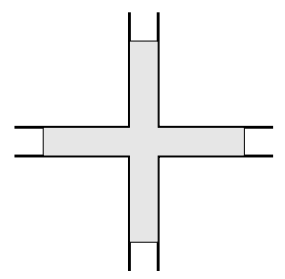

b)
FIG. 16. Examples of four-terminal disordered conductors. Disordered area is shaded. Dashed line denotes diffusive motion between its ends.

Once we determined that exchange correction $\Delta S$ exists in diffusive conductors, we must evaluate its sign and relative magnitude. We only describe the results qualitatively; details can be found in Refs. 778,114. Two specific geometries have been investigated: the disordered box (Fig. 16a) and the disordered cross (Fig. 16b). For the box, the exchange correction $\Delta S$ is negative, i.e. exchange suppresses noise $\left(S_{C}<S_{A}+S_{B}\right)$. The effect is quite considerable: The correction is of the same order of magnitude as the classical contributions in $S_{A}$ and $S_{B}$, and is suppressed only by a numerical factor. For the cross, the exchange contribution is positive - exchange enhances noise - but the magnitude is by powers of $l / L$ smaller than $S_{A}$ and $S_{B}$. Here $l$ and $L$ are the mean free path and the length of the disordered arms, respectively. Thus, neither sign nor magnitude of the exchange effects is predetermined in diffusive systems: they are geometry and disorder dependent, and the only limitation is $S_{C}>0$.

Gramespacher and one of the authors 110,111 considered a geometry of a disordered wire (along the axis $z$ ) between the contacts $1(z=0)$ and $3(z=L)$, coupled locally at the points $z$ and $z^{\prime}$ to the contacts 2 and 4 , respectively, via high tunnel barriers (these latter can be viewed as scanning tunneling microscope tips) $\lcm{27}$ and evaluated Eq. (109). It was found that the exchange effect is positive in the case, i.e. it enhances noise, irrespectively of the position of the contacts 2 and 4 . For the particular case when both tunnel contacts are situated symmetrically around the center of the wire at a distance $d, z=(L-d) / 2$ and $z^{\prime}=(L+d) / 2$, the relative strength of the exchange term is

$$
\frac{\Delta S}{S_{C}}=\frac{1}{3}\left[2+\frac{d}{L}-2\left(\frac{d}{L}\right)^{2}\right],
$$

and reaches its maximum for $d=L / 4$. We see that the exchange effect in this case generally has the same order of magnitude as the classical terms $S_{A}$ and $S_{B}$.

Chaotic cavities. A similar problem in chaotic cavities was addressed in Ref. [115 (see also Ref. [116]). Similarly to disordered systems, it was discovered that exchange effects survive on average. An additional feature is however that the exchange effects in chaotic cavities are universal. That of course is a consequence of the assumption that the cavity can be described by using Dyson's circular ensemble. For open cavities, one finds

$$
\Xi_{1}=\Xi_{2}=-3 \Xi_{3}=-3 \Xi_{4}=\frac{3}{4} \frac{N_{\perp}^{3}}{16 N_{\perp}^{2}-1},
$$

where we assumed that all leads are identical and support $N_{\perp}$ transverse channels. This implies $S_{A}=S_{B}$, $S_{C}=4 S_{A} / 3$, or $\Delta S=-2 S_{A} / 3$. Thus, exchange effects suppress noise in open chaotic cavities.

The situation changes if the cavity is separated from the leads by tunnel barriers. Assuming that the transmission coefficients of all barriers in all transverse channels are identical and equal $T, N_{\perp} T \gg 1$, Ref. [115] finds

$$
\left\{\begin{array}{c}
\Xi_{1}=\Xi_{2} \\
\Xi_{3}=\Xi_{4}
\end{array}\right\}=\frac{N_{\perp} T}{64}\left\{\begin{array}{c}
T+2 \\
-3 T+2
\end{array}\right\} .
$$

Thus, for $T=2 / 3$ the exchange effect changes sign: If the barriers separating the cavity from the reservoirs are high enough, the exchange enhances the correlations. In the limit of very opaque barriers, $T \rightarrow 0$, we have $S_{C}=$ $2\left(S_{A}+S_{B}\right)$ : the exchange correction is the same as the classical contributions $S_{A}$ and $S_{B}$.

Edge channels in the quantum Hall effect regime. Interesting tests of exchange (interference) effects can also be obtained in high magnetic fields using edge channels. In fact this leads to a simple example

\footnotetext{
${ }^{27}$ Ref. 111 also considers a three-terminal structure (a (disordered) wire with a single STM tip attached to it). The fluctuations of the current through the tip are in this case proportional to the local distribution function of electrons at the coupling point, see Eq. (249) below.
} 
where the phase dependence of the exchange effect is indeed essential [9]. Imagine that the system is placed into a strong magnetic field, and the transport is only due to edge channels. We assume that there is only one edge state flowing from 1 through 2 and 3 to 4 and back to 1 (Fig. 17). Furthermore, for simplicity we assume that all the leads are identical, and the transmission probability to enter from the lead to the edge state is $T$. A direct calculation gives [9]

$$
\begin{aligned}
& \Xi_{1}=\Xi_{2}=\frac{T^{4}(1-T)^{2}}{\left[1+(1-T)^{4}-2(1-T)^{2} \cos \phi\right]^{2}}, \\
& \Xi_{3}=\Xi_{4}^{*}=\frac{T^{4}(1-T)^{2} \exp (i \phi)}{\left[1+(1-T)^{4}-2(1-T)^{2} \cos \phi\right]^{2}},
\end{aligned}
$$

where $\phi$ is the phase accumulated along the whole trajectory, and the phase dependence in the denominator appears due to the possibility of multiple traversals of the full circle. We have

$S_{C}=\frac{2 e^{3}|V|}{\pi \hbar} \frac{T^{4}(1-T)^{2}}{\left[1+(1-T)^{4}-2(1-T)^{2} \cos \phi\right]^{2}}(1-\cos \phi)$.

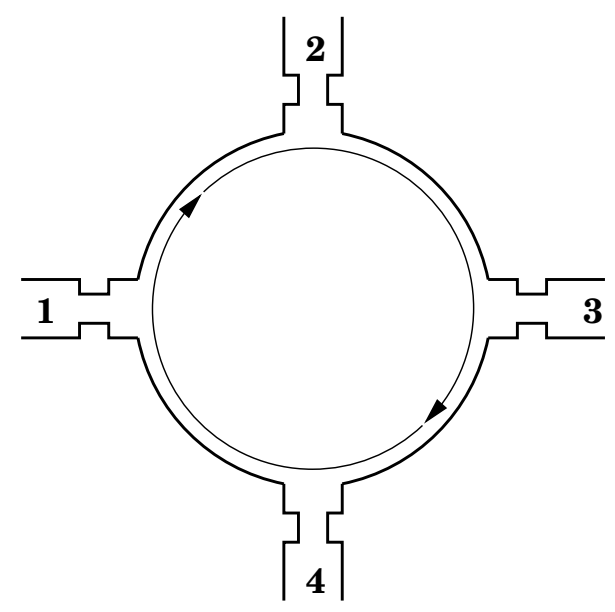

FIG. 17. Hanbury Brown - Twiss effect with edge states.

Here the term with 1 represents the "classical" contributions $S_{A}+S_{B}$, while that with $\cos \phi$ is accountable for the exchange effect $\Delta S$. We see that, depending on $\phi$, exchange effects may either suppress (down to zero, for $\phi=0$ ) or enhance (up to $2\left(S_{A}+S_{B}\right)$, for $\phi=\pi$ ) total noise. This is an example illustrating the maximal phase sensitivity which the exchange effect can exhibit.

This simple example gives also some insight on how exchange effects survive ensemble averaging. Since the phase $\phi$ occurs not only in the numerator but also in the denominator, the average of Eq. (111) over an ensemble of cavities with the phase $\phi$ uniformly distributed in the interval from 0 to $2 \pi$ is non-zero and given by

$$
\left\langle S_{C}\right\rangle=\frac{2 e^{3}|V|}{\pi \hbar} \frac{T^{4}(1-T)^{2}}{\left[1-(1-T)^{2}\right]^{3}\left[1+(1-T)^{2}\right]} .
$$

The ensemble averaged exchange contribution vanishes both in the limit $T=0$ and in the limit $T=1$.

Note that if another order of contacts is chosen, $1 \rightarrow$ $3 \rightarrow 2 \rightarrow 4$, the whole situation changes: the exchange term is now phase insensitive and has a definite sign (negative, i.e. exchange suppresses noise) [9]. This is because the trajectories responsible for exchange terms do not form closed loops in this case.

Experiments. The phase-sensitive Hanbury BrownTwiss effect discussed above has not so far been probed in experiments. However, a related experiment was carried out by Liu et al [117, 118], who measured the mean squared fluctuations $S_{33}$ of the current in the lead 3, of a four probe structure, applying voltages in the same three-fold ways that we have discussed.

Prior to the description of experimental results, we discuss briefly a measurement of $S_{33}$ on the quantum Hall conductor of Fig. 13. If current is incident from contact 4 (experiment A), or contact 2 (experiment B) alone we have for the current fluctuations at contact 3 $S_{33}^{A}=S_{33}^{B}=\left(e^{3}|V| / \pi \hbar\right) T(1-T)$. On the other hand if currents are incident both from contact 4 (experiment C) and contact 2, all states are now completely filled and thus in the zero-temperature limit $S_{33}^{C}=0$. Thus, in comparison to experiments A or B there is a complete reduction of the shot noise at contact 3 in experiment $\mathrm{C}$ : The spectral density $S_{33}^{C}$ is suppressed down to zero.

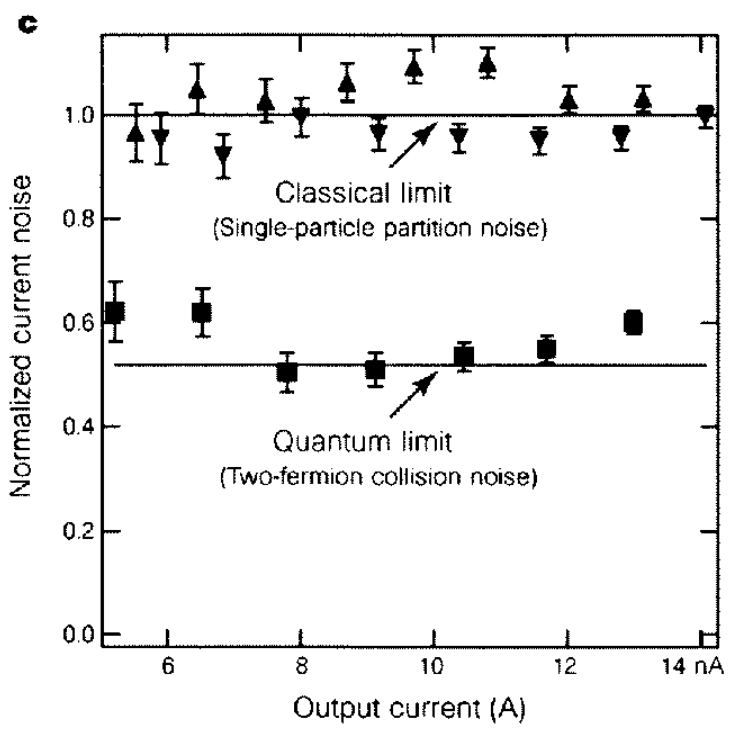

FIG. 18. Experimental results of Liu et al 118 . Upward and downward triangles correspond to the situations when only one of the two input contacts is open (values $S_{A}$ and $S_{B}$, respectively); squares indicate the case when both input contacts are effective $\left(S_{C}\right)$.

In the experiment of Liu et al 117,118] the mean square current fluctuations are measured in zero magnetic field in a conductor in which a left input (1) and output contact (3) are separated by a thin barrier from a right input contact (2) and output contact (4) (see Fig. 
18). The input contacts form QPC's and are adjusted to provide transmission close to 1 . The output contacts support a number of channels. In this experiment it thus not possible to fill all outgoing states in contact 3 completely and there is thus only a limited reduction of noise in experiment $\mathrm{C}$ compared to experiments $A$ and $B$. The experimentally observed ratio $S_{C} /\left(S_{A}+S_{B}\right)=0.56$.

It is also useful to compare the experiment of Liu et al 117, 118 simply with a chaotic cavity connected to point contacts which are fully transparent $T=1$ 115]. Then using Eq. 1110) one finds a ratio $S_{C} /\left(S_{A}+S_{B}\right)=2 / 3$ which is surprisingly close to what was observed in the experiment.

\section{Aharonov - Bohm effect}

The Aharonov - Bohm (AB) effect tests the sensitivity to a magnetic flux $\Phi$ of electrons on a trajectory which enclose this flux. In the pure AB-effect the electron does not experience the magnetic field, the electron trajectory is entirely in a field free region. It is a genuine quantum effect, which is a direct consequence of the gauge invariance of the velocity and the wave nature of electrons. The simplest geometry demonstrating the $\mathrm{AB}$ effect in electric transport is a ring coupled to two reservoirs and threaded by a magnetic flux, as shown in Fig. 19a. Then, the $\mathrm{AB}$ effect is manifest in a periodic flux dependence of all the transport properties.

Qualitatively different phenomena arise in weak and strong magnetic fields, and these two cases need to be considered separately.

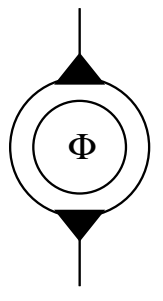

a)

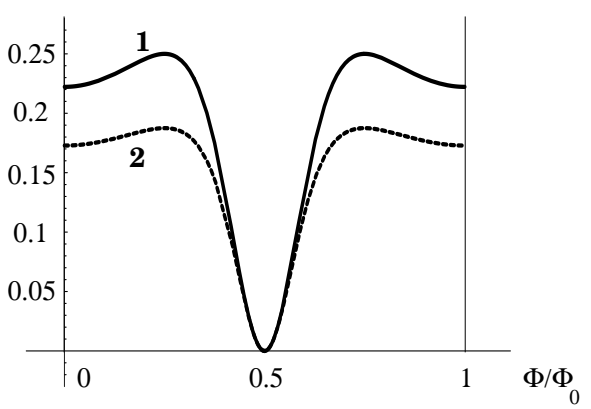

b)
FIG. 19. (a) Geometry of a ring threaded by a flux $\Phi$, demonstrating the Aharonov-Bohm effect in a weak magnetic field. (b) Conductance in units of $e^{2} / 2 \pi \hbar$ (1) and shot noise power in units of $e^{3} V / \pi \hbar$ (2) as a function of the $\mathrm{AB}$ flux $\Phi / \Phi_{0}$ for a particular value of the phase $\phi=\pi / 2$.

Weak magnetic fields. In this regime, the transmission coefficient(s) (and, subsequently, conductance) of the two-terminal structure shown in Fig. 19a, is a periodic function of the external flux, with the period $\Phi_{0}=2 \pi \hbar c / e$. The resulting conductance is samplespecific, and, in particular, it is very sensitive to the phase of the trajectory enclosing the flux. In phase coherent many-channel conductors the AB-oscillations in the conductance represent a small correction to a flux insensitive (classical) background conductance 2 . Thus the $\mathrm{AB}$ effect is most dramatic in single channel rings [120,121 where the flux induced modulations of the conductance are of the order of the conductance itself. Shot noise in such a structure was studied by Davidovich and Anda 122 using the nonequilibrium Green's functions technique. They considered a one-channel ring and used a tight-binding description of the ring and leads. Here we will give another derivation, based on the scattering approach. A related issue was discussed by Iannaccone, Macucci, and Pellegrini [123], who studied noise in a multiply-connected geometry using the scattering approach, and found that if there is no transmission from the left part of the ring to the right part and vice versa (Fig. 19a), noise of the left and right parts add up classically. In particular, this means that such a system would not exhibit an $\mathrm{AB}$ effect.

We follow Refs. 120,121 which study the transmission coefficient of single-channel rings connected to external leads (Fig. 19a). Our purpose here is to illustrate only the principal effect, and therefore we consider the simple case without scattering in the arms of the ring. We also assume that the ring is symmetric. Formulae for shot noise in more complicated situations can be readily produced from Ref. [120,121, though, to the best of our knowledge, they have never been written down explicitly.

We describe the "beam splitters", separating the leads from the ring (black triangles in Fig. 19a) by the scattering matrix 121

$$
s_{b}=\left(\begin{array}{ccc}
-(a+b) & \epsilon^{1 / 2} & \epsilon^{1 / 2} \\
\epsilon^{1 / 2} & a & b \\
\epsilon^{1 / 2} & b & a
\end{array}\right),
$$

where the parameter $\epsilon, 0<\epsilon<1 / 2$, is responsible for the coupling of the ring to the lead, and

$$
\begin{aligned}
& a=\frac{1}{\sqrt{2}}(\sqrt{1-2 \epsilon}-1), \\
& b=-\frac{1}{\sqrt{2}}(\sqrt{1-2 \epsilon}+1) .
\end{aligned}
$$

Specializing to the case of the ring which is ideally coupled to the leads, $\epsilon=1 / 2$, we obtain for the transmission coefficient 121]

$$
T(\Phi)=\frac{(1+\cos \theta) \sin ^{2} \phi}{(1+\cos \theta-\cos 2 \phi)^{2}+(1 / 2) \sin ^{2} 2 \phi},
$$

\footnotetext{
${ }^{28}$ In disordered systems, the ensemble averaged conductance exhibits $\mathrm{AB}$ oscillations with the period of $\Phi_{0} / 2$. These oscillations are, like the weak localization correction, associated with the interference of two electron trajectories running in opposite directions 119. Weak localization effects and shot noise have not so far been investigated.
} 
where $\theta=2 \pi \Phi / \Phi_{0}$, and $\phi$ is the phase accumulated during the motion along a half of the ring (without magnetic field). Now the conductance (40) $G=\left(e^{2} / 2 \pi \hbar\right) T$ and the shot noise (57) $S=\left(e^{3}|V| / \pi \hbar\right) T(1-T)$ are immediately expressed as functions of the applied magnetic flux. They are strongly dependent on the phase $\phi$, which is sample-specific. In particular, both the conductance and the shot noise vanish for $\phi=0$ or $\phi=\pi$. This is a consequence of the symmetry assumed here: If the leads are attached asymmetrically to the ring the transmission coefficient stays finite for any value of the phase [124]. The dependence of conductance and shot noise on flux $\Phi$ for a particular value $\phi=\pi / 2$ is shown in Fig. 19b. We reemphasize that the flux dependence shown depends strongly on the sample specific phase $\phi$.

Strong magnetic fields. Now we turn to the situation of the quantum Hall effect, where transport current is carried by the edge states. A remarkable feature of this regime is that a two-terminal ring without backscattering does not exhibit the $\mathrm{AB}$ effect. The edge states (Fig. 20a) exist in different regions of space, and thus do not interfere. Indeed, the absence of backscattering, which precludes the AB-effect, is just the condition for conductance quantization 102. We cannot have both a quantized conductance and an $\mathrm{AB}$-effect.

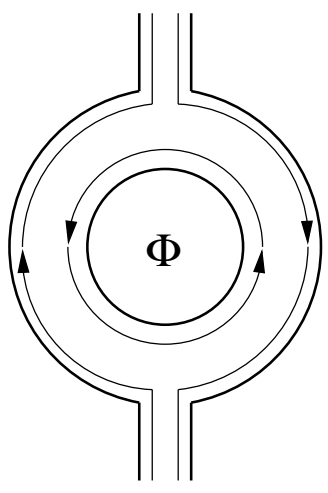

a)

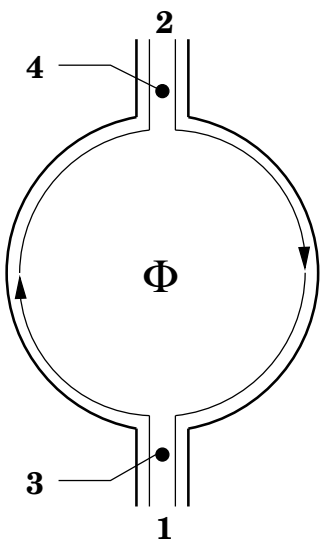

b)
FIG. 20. (a) Geometry of a ring in strong magnetic field. Edge states are shown. (b) Four-terminal geometry which facilitates separation of scattering and AB effects.

The question which we want now to address is the following: Can one observe $\mathrm{AB}$ effects in the noise, which the fourth order interference effect in a situation when they do not exist in the conductance which is only a second order interference effect? We noticed already that shot noise is a phase sensitive effect, and contains interference terms, absent in the conductance. The shot noise contains non-real terms composed of four scattering matrix elements. This is the case already in the two-terminal shot noise formula when it is expressed in the natural basis. In the two terminal case the appearance of such products depends, however, on the basis we chose: The shot noise is a function of transmission probabilities only, if it is evaluated in the eigen channel basis. However, in a multi-terminal geometry, such products appear naturally if we consider current-current cross-correlations. We call these non-real products exchange interference terms, since they are a manifestation of interference effects in multi-particle wave functions (Slater determinants of single particle wave functions) which result from the indistinguishability of carriers. In contrast, the effects we have already seen in weak fields, which contribute to the conductance as well as to the shot noise are a consequence of second order or direct interference.

The two-terminal geometry of Fig. 20a is not appropriate for the observation of the exchange interference effects, since shot noise vanishes without scattering between edge channels. If scattering is introduced, shot noise becomes finite, but at the same time the conductance becomes sensitive to the flux, due to direct interference. One can try to separate $\mathrm{AB}$ effects in the shot noise due to direct and exchange interference, but this is awkward.

A possible way out was proposed in Ref. [112], which suggested four-terminal geometries with two weak coupling contacts. We follow here a subsequent, clearer discussion given in Ref. 111]. The geometry is shown in Fig. 20 b. This is a quantum dot in a strong magnetic field coupled via two quantum point contacts to reservoirs. The ring geometry is actually not needed in the experiment and serves only for conceptual clarity. Current flows between contacts 1 and 2, and the contacts 3 and 4 are inserted locally at the quantum point contact between the edge states in the leads. The magnetic field is such that the two-probe conductance is quantized, but weak enough such that at the quantum point contact the left- and right-going wave functions of the two edge channels overlap. As is well known, the fact that the wave functions in the quantum point contact overlap, does not destroy the quantization, as long as the potential of the quantum point contact is smooth. We take the scattering matrix relating the amplitudes of carriers in the contact 3 (or 4) with those in the edge channels nearby to be of the same form (113) as for the "beam splitter" discussed above. Here it is now essential to assume that coupling is weak thus we take $\epsilon \ll 1$. In the experiment proposed in Refs. 112,111 the same voltage $V$ is applied simultaneously to the contacts 1 and 2 . Then we have

$$
\begin{aligned}
& S_{33}=S_{44}=\frac{2 e^{3}|V|}{\pi \hbar} \epsilon, \\
& S_{34}=-\frac{2 e^{3}|V|}{\pi \hbar} \epsilon^{2}\left[1+\cos \left(\phi+2 \pi \Phi / \Phi_{0}\right)\right],
\end{aligned}
$$

where $\phi$ is a certain phase. The relative value of $S_{34}$ as compared to $S_{33}$ is $\epsilon$. At the same time, the corrections to the conductance and the shot noise due to direct interference are proportional to $\epsilon^{2}$. Thus, in our geometry up to the terms of $\epsilon^{2}$ conductance is not renormalized by the $\mathrm{AB}$ effect, while shot noise feels it due to its two-particle 
nature. This is thus a geometry where the AB effect manifests itself in the fourth order interference and modulates the Hanbury Brown - Twiss effect (the current-current cross-correlation at contacts 3 and 4 ).

\section{G. Inelastic scattering. Phase breaking}

Throughout this Section, we treated the mesoscopic systems as completely phase coherent. In reality, there is always at least some inelastic or phase breaking scattering present. The scattering approach as it was used here, is based on the carrier transmission at a definite energy. In contrast, electron-electron or electron-phonon interactions can change the energy of a carrier. Thus a scattering theory of such processes has to be based on a scattering amplitudes which permit incoming and outgoing particles to have different energies. To our knowledge, the extension of scattering theory of electrical transport within such a generalized scattering matrix approach has not been worked out. It is, however, possible to make progress even within the scattering approach used so far: To treat phase breaking theoretically we often proceed by inventing a Hamiltonian system with many degrees of freedom while we are interested in the behavior of only a subsystem. Similarly it is possible to arrive at an approach which describes inelastic transitions and phase breaking by first considering a completely phase-coherent conductor with one or a continuum of additional voltage probes which are purely fictitious [125, 47]. The additional fictitious voltage probes act as dephasers on the actual conductor of interest. This approach has been widely used to investigate the effect of dephasing on conductance. We refer the reader here only to a few early works 125, 47, 126, 127. In this subsection we illustrate the application of these ideas to noise. Other approaches, based on Green's function techniques, have also been invoked to derive results for strongly correlated systems (see Section VII). Furthermore, on the purely classical level, it proved to be rather simple to extend the fluctuating Boltzmann equation approach to include interactions. For the results on interaction and noise in double barrier resonant tunneling structures and metallic diffusive conductors the reader is addressed to Sections $\mathrm{V}$ and $\mathrm{VI}$, respectively. The approach which uses voltage probes as dephasers is interesting because of its conceptual clarity and because of its close relation to experiments: The effect of additional voltage probes can easily be tested experimentally with the help of gates which permit to switch off or on a connection to a voltage probe (see e.g. Ref. 128).

Voltage probes as dephasers. Consider a mesoscopic conductor connected to $N$ (real) contacts. To introduce inelastic scattering, we attach a number $M$ of purely fictitious voltage probes to this conductor. The entire conductor with its $N+M$ contacts is phase coherent and exhibits the noise of a purely phase coherent conductor. However, elimination of the $M$ fictitious voltage probes leads to an effective conduction problem for which the conductance and the noise depend on inelastic scattering processes 19,74,129 131. Depending on the properties of the fictitious voltage probes, three different types of inelastic scattering can be realized, which de Jong and Beenakker 90] classify as "quasi-elastic scattering" (phase breaking), "electron heating", and "inelastic scattering". Now we describe these types of probes separately. This division corresponds to the distinction of $\tau_{\phi}$, $\tau_{e e}$, and $\tau_{i n}$. We emphasize that only a microscopic theory can give explicit expressions for these times. What the approach based on fictitious voltage probes can do is to find the functional dependence of the conductance or the noise on these times.

The results for interaction effects in double-barrier structures seem to be well established by now. In contrast, for diffusive metallic wires with interactions the situation is less clear. For discussion, the reader is addressed to Section VI.

In this subsection, we assume that the system is charge neutral, i.e. there is no pile-up of charge. This charge neutrality is normally provided by Coulomb interactions, which thus play an important role. If this is not the case, one can get different results, like for resonant tunneling quantum wells with charging (Section V) or quantum dots in the Coulomb blockade regime (Section VII).

Inelastic scattering. We begin the discussion with the strongest scattering processes which lead to carrier energy relaxation and consequently also energy dissipation. Physically, this may correspond to electron-phonon scattering. To simulate this process, we consider a twoterminal structure in the conceptually simple case where we add only one fictitious voltage probe 29 (marked as 3, see Fig. 21). As in our treatment of noise in multiprobe conductors we assume that all reservoirs (also the voltage probe reservoir 3) are characterized by Fermi distribution functions (for simplicity, we only consider zerotemperature case). We take $\mu_{2}=0$ and $\mu_{1}=e V$; the chemical potential $\mu_{3}$ is found from the condition that at a voltage probe the current $I_{3}$ vanishes at any moment of time. Then an electron which has left the conductor and escaped into the reservoir of the voltage probe must immediately be replaced by another electron that is reinjected from the voltage probe into the conductor with an energy and phase which are uncorrelated with that of the escaping reservoir. This approach to inelastic scattering was applied to noise in interacting mesoscopic systems in Ref. 19] (where only the average current was

\footnotetext{
${ }^{29}$ There are a number of works treating the effect of distributed dephasing (many voltage probes) on conduction processes (see for instance Ref. 132). Results on inelastic scattering on shot noise with several probes can be found in Refs. $74,82,90$.
} 
taken to vanish), and the analysis for an instantaneously vanishing current was presented in Ref. [74].
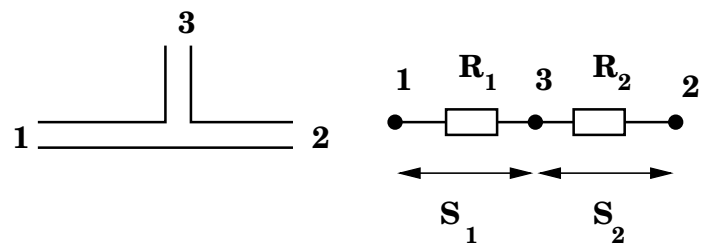

a)

b)
FIG. 21. (a) Setup with intermediate electrode. (b) Equivalent circuit for the case when there is no direct transmission from 1 to 2 .

To proceed, we introduce transmission probabilities from the lead $\alpha$ to the lead $\beta$,

$$
T_{\alpha \beta}=\operatorname{Tr} s_{\alpha \beta}^{\dagger} s_{\alpha \beta}, \quad \sum_{\beta} T_{\alpha \beta}=\sum_{\beta} T_{\beta \alpha}=N_{\alpha},
$$

$N_{\alpha}$ being the number of transverse channels in the lead $\alpha$. Currents can be then written as

$$
\begin{aligned}
& I_{1}=\frac{e^{2}}{2 \pi \hbar}\left[T_{12} e V+T_{13}\left(e V-\mu_{3}\right)\right]+\delta I_{1}, \\
& I_{2}=\frac{e^{2}}{2 \pi \hbar}\left[-T_{21} e V-T_{23} \mu_{3}\right]+\delta I_{2}, \\
& I_{3}=\frac{e^{2}}{2 \pi \hbar}\left[-T_{31}\left(e V-\mu_{3}\right)+T_{32} \mu_{3}\right]+\delta I_{3},
\end{aligned}
$$

where $\delta I_{\alpha}$ are the fluctuating parts of the currents (each of them is zero on average), correlated according to Eq. (55) with $\mu_{3}$ replaced by the average $\left\langle\mu_{3}\right\rangle$. The current conservation implies $\delta I_{1}+\delta I_{2}+\delta I_{3}=0$. The requirement $I_{3}=0$ implies now that the electrochemical potential at the voltage probe is a fluctuating function of time $\mu_{3}(t)$ given by

$$
\mu_{3}=\frac{T_{31}}{T_{31}+T_{32}} e V+\frac{2 \pi \hbar}{e^{2}} \frac{\delta I_{1}+\delta I_{2}}{T_{31}+T_{32}} .
$$

The first term on the right hand side represents the average chemical potential $\left\langle\mu_{3}\right\rangle$, while the second one is a fluctuating correction. The two-terminal conductance (defined according to $\left\langle I_{2}\right\rangle=G V$ ) depends only on the average potential $\left\langle\mu_{3}\right\rangle$ and is given by 125,47]

$$
G=\frac{e^{2}}{2 \pi \hbar}\left[T_{12}+\frac{T_{13} T_{23}}{T_{13}+T_{23}}\right]
$$

In Eq. (116) the probability $T_{12}$ describes coherent transmission, whereas the second term is the incoherent contribution.

Due to the fluctuations of the chemical potential $\mu_{3}$, the random part of the current $I_{1}$ is now

$$
\Delta I_{1}=\delta I_{1}-\frac{T_{13}}{T_{31}+T_{32}}\left(\delta I_{1}+\delta I_{2}\right)
$$

and due to the current conservation the random part of the current $I_{2}$ is the same with the opposite sign. Now the expression for the shot noise power $S_{11}$ can be easily obtained from Eq. (5.5), but it is rather cumbersome in the general case. In the following, we consider only the fully incoherent case $T_{12}=0$, when there is no direct transmission from 1 to 2: Every carrier on its way from contact 1 to 2 enters the electrode 3 with the probability one. This condition also implies $T_{\alpha 3}=T_{3 \alpha}$. Essentially, the fully incoherent case means that the two parts of the system, from 1 to 3 , and from 3 to 1 , are resistors which add classically. In particular, the conductance Eq. (116) contains now only the second term, which now just states that two consecutive incoherent scatterers exhibit a resistance which is equal to the series resistance. For the current correlations, we obtain $S_{11}=S_{22}=-S_{12}=$ $-S_{21}$ with

$S_{11}=\frac{e^{3}|V|}{\pi \hbar} \frac{T_{23}^{2} \operatorname{Tr}\left[s_{11} s_{11}^{\dagger} s_{13} s_{13}^{\dagger}\right]+T_{13}^{2} \operatorname{Tr}\left[s_{22} s_{22}^{\dagger} s_{23} s_{23}^{\dagger}\right]}{\left(T_{13}+T_{23}\right)^{3}}$.

This expression can be re-written in the following transparent manner: First, we define the resistances of the parts of the system between 1 and $3, R_{1}=$ $2 \pi \hbar /\left(e^{2} T_{13}\right)$, and between 2 and $3, R_{2}=2 \pi \hbar /\left(e^{2} T_{23}\right)$. The total resistance between 1 and 2 is given by $R=$ $R_{1}+R_{2}$. Now the voltage drop between 1 and 3 is $e V R_{1} / R$ and between 3 and 2 is $e V R_{2} / R$. Taking this into account, the noise power measured between 1 and 3 is (Fig. 21),

$$
S_{1}=\frac{e^{3}|V|}{\pi \hbar} \frac{R_{1}}{R} \operatorname{Tr}\left[s_{13} s_{13}^{\dagger}\left(1-s_{13} s_{13}^{\dagger}\right)\right],
$$

and noise power measured between 2 and 3 is,

$$
S_{2}=\frac{e^{3}|V|}{\pi \hbar} \frac{R_{2}}{R} \operatorname{Tr}\left[s_{23} s_{23}^{\dagger}\left(1-s_{23} s_{23}^{\dagger}\right)\right] .
$$

Now we can write Eq. (117) as 74$]\left(S \equiv S_{11}\right)$

$$
R^{2} S=R_{1}^{2} S_{1}+R_{2}^{2} S_{2} .
$$

The meaning of Eq. (118) is obvious if we realize that $R^{2} S$ is the voltage fluctuations (for an infinite external impedance circuit) across the whole conductor. The right hand side is just a sum of voltage fluctuations from 1 and 3 , and from 3 to 2 . Thus, Eq. (118) states nothing but that the voltage fluctuations are additive.

Another form of Eq. (118) is useful 133. . We introduce the noise suppression factors $F_{1}=S_{1} R /(2 e|V|)$ and $F_{2}=$ $S_{2} R /(2 e|V|)$ in the first and second resistor. For the Fano factor of the whole system we obtain

$$
F=\frac{R_{1}^{2} F_{1}+R_{2}^{2} F_{2}}{\left(R_{1}+R_{2}\right)^{2}} .
$$

First of all, we now evaluate Eq. (119) for the case of the double-barrier structure, where the intermediate 
electrode is placed between the barriers. Physically, this would correspond to strong inelastic scattering inside the quantum well - in contrast to the quantum-mechanical discussion of the previous subsection, which implicitly requires full phase coherence. Taking into account that for high barriers $F_{1}=F_{2}=1$, and that the resistances $R_{1}$ and $R_{2}$ are inversely proportional to the tunneling rates $\Gamma_{L}$ and $\Gamma_{R}$, respectively, we immediately arrive at Eq. (78), i.e. the result for the fully coherent case $\mathrm{g}^{30}$. We thus see that even though inelastic scattering modifies both the conductance and shot noise of the resonant tunneling structure, it leaves the Fano factor unchanged. This statement, due to Chen and Ting [134] and Davies et al [54, will be again demonstrated in Section $\mathrm{Q}$, where the derivation of Eq. (78) based on a classical Langevin approach (which corresponds to the absence of quantum coherence) is presented.

Next we consider a quasi-one-dimensional geometry and assume for a moment that the lead 3 divides the wire into two identical parts. Then in Eq. (119) $R_{1}=R_{2}$, $F_{1}=F_{2}$, and we obtain $F=F_{1} / 2$. Thus, the Fano factor of the whole wire is one half of the noise measured in each segment.

This result, which describes local inelastic scattering in the middle of the wire, can be generalized to uniform inelastic scattering. For this purpose we introduce a certain length $L_{i}$ associated with inelastic scattering; we assume that $L_{i}$ is much shorter than the total length of the wire $L$. One must then consider initially a conductor with $N_{i}=L / L_{i}$ additional fictitious voltage probes separated by distances $L_{i}$ along the conductor. In the fully incoherent case this picture is equivalent to $N_{i}$ identical classical resistors connected in series. The Fano factor of this system is then the Fano factor of the phase-coherent segment divided by $N_{i}$. In particular, if the wire is diffusive, the suppression factor ${ }^{31}$ is $77,82, F=\left(3 N_{i}\right)^{-1}$. Thus, the conclusion is the following: Inelastic scattering suppresses shot noise. A macroscopic system (large compared to an inelastic scattering length, $N_{i} \gg 1$ ) exhibits no shot noise. This is a well known fact, the absence of shot noise of macroscopic conductors is used to stabilize lasers.

Shimizu and Ueda 133 and Liu and Yamamoto

\footnotetext{
${ }^{30}$ Lund $\mathrm{B} \varnothing$ and Galperin 59 performed microscopic calculation of noise in quantum wells in transverse magnetic field with the account of electron-phonon scattering (phononassisted tunneling). They found that the Fano factor is suppressed by inelastic scattering, as compared with noninteracting value. This result clearly contradicts to the conclusions of this subsection; we presently do not understand the reasons for this discrepancy.

${ }^{31}$ Of course, not only the average of the noise vanishes in macroscopic system, but also fluctuations. De Jong and Beenakker found r.m.s. $S \propto N_{i}^{-5 / 2}$. The weak localization correction to shot noise decreases as $N_{i}^{-2}$.
}

[129,130, provided a similar discussion of noise suppression in the crossover regime between mesoscopic behavior and classical circuit theory (macroscopic behavior). Liu, Eastman, and Yamamoto [91] performed Monte Carlo simulations of shot noise and included explicitly electronphonon scattering.

Quasi-elastic scattering. In contrast to inelastic scattering, dephasing processes leave the energy essentially invariant. To simulate a scattering process which destroys phase but leaves the energy invariant we now have to consider a special voltage probe. We require that the additional electrode conserves not only the total current, but also the current in each small energy interval [90]. Such a voltage probe will not give rise to energy relaxation and dissipates no energy.

From the condition that the current in each energy interval vanishes we find that the distribution function in the reservoir of the voltage probe is given by

$$
f_{3}(E)=\frac{G_{1} f_{1}(E)+G_{2} f_{2}(E)}{G_{1}+G_{2}},
$$

where $f_{1}$ and $f_{2}$ are the Fermi functions at the reservoirs 1 and 2 , and the conductances are $G_{1}=R_{1}^{-1}, G_{2}=R_{2}^{-1}$. We have assumed again that there is no direct transmission between 1 and 2. Straightforward calculation gives for the Fano factor 90

$$
F=\frac{R_{1}^{3} F_{1}+R_{2}^{3} F_{2}+R_{1}^{2} R_{2}+R_{1} R_{2}^{2}}{\left(R_{1}+R_{2}\right)^{3}} .
$$

We analyze now this result for various situations. First, we see that for a ballistic wire divided by a dephasing electrode into two parts, $F_{1}=F_{2}=0$, shot noise does not vanish (unlike Eq. (121)). We obtain $F=$ $R_{1} R_{2}\left(R_{1}+R_{2}\right)^{-2}$. Thus for a ballistic system, which is ideally noiseless, dephasing leads to the appearance of shot noise.

For the strongly biased resonant double-barrier structure, we have $F_{1}=F_{2}=1$, and obtain again the result (78), which is thus insensitive to dephasing.

For metallic diffusive wires, $F_{1}=F_{2}=1 / 3$, Eq. (121) yields for the ensemble averaged Fano factor $F=1 / 3$ independent on the location of the dephasing voltage probe, i. e. independent of the ratio of $R_{1}$ and $R_{2}$. Thus, our consideration indicates that the noise suppression factor for metallic diffusive wires is also insensitive to dephasing, at least when the dephasing is local (in any point of the sample). This result hints that the Fano factor of an ensemble of metallic diffusive wires is not sensitive to dephasing even if the latter is uniformly distributed. Indeed, de Jong and Beenakker [82] checked this by coupling locally a dephasing reservoir to each point of the sample. Already at the intermediate stage their formulae coincide with those obtained classically by Nagaev [75]. This proves that introducing dephasing with fictitious, energy conserving voltage probes is in the limit of complete dephasing equivalent to the Boltzmann-Langevin approach. That inelastic scattering, and not dephasing, 
is responsible for the crossover to the macroscopic regime, has been recognized by Shimizu and Ueda 133.

The effect of phase breaking on the shot noise in chaotic cavities was investigated by van Langen and one of the authors 115. For a chaotic cavity connected to reservoirs via quantum point contacts with $N_{L}$ and $N_{R}$ open quantum channels, the Fano factors vanish $F_{1}=$ $F_{2}=0$, and since $R_{1}=\pi \hbar / e^{2} N_{L}$ and $R_{2}=\pi \hbar / e^{2} N_{R}$, the resulting Fano factor is given by Eq. (96), i.e. it is identical with the result that is obtained from a completely phase coherent, quantum mechanical calculation. Thus for chaotic cavities, like for metallic diffusive wires, phase breaking has no effect on the ensemble averaged noise power.

Electron heating. This is the third kind of inelastic scattering, which implies that energy can be exchanged between electrons. Only the total energy of the electron subsystem is conserved. Physically, this corresponds to electron-electron scattering. Within the voltage probe approach, it is taken into account by including the reservoir 3 , with chemical potential $\mu_{3}$ determined to obtain zero (instantaneous) electrical current and a temperature $T_{3}$, which is generally different from the lattice temperature (or the temperature of the reservoirs 1 and 2), to obtain zero (instantaneous) energy flux. For a detailed discussion we refer the reader to the paper by de Jong and Beenakker [82], here we only mention the result for two identical diffusive conductors at zero temperature 2 . The Fano factor is in this case $F \approx 0.38$, which is higher than the $1 / 3$-suppression for the non-interacting case. We will see in Section $\mathrm{V1}$ that the classical theory also predicts shot noise enhancement for the case of electron heating.

Intermediate summary. Here are the conclusions one can draw from the simple consideration we presented above.

- Dephasing processes do not renormalize the ensemble averaged shot noise power (apart from weak localization corrections, which are destroyed by dephasing). In particular, this statement applies to metallic diffusive wires, chaotic cavities, and resonant double barrier structures.

- Inelastic scattering renormalizes even the ensemble averaged shot noise power: A macroscopic sample exhibits no shot noise. An exception is the resonant double barrier structure, subject to a bias large compared to the resonant level width. Under this condition neither the conductance nor the shot noise of a double barrier are affected.

- As demonstrated for metallic diffusive wires electron heating enhances noise.

\footnotetext{
${ }^{32}$ The temperature of the reservoirs 1 and 2 is zero. The temperature of the intermediate reservoir is in this case 82 $k_{B} T_{3}=(\sqrt{3} / 2 \pi) e|V|$.
}

The last statement implies the following scenario for noise in metallic diffusive wires 80]. There exist three inelastic lengths, responsible for dephasing $\left(L_{1}\right)$, electron heating $\left(L_{2}\right)$ and inelastic scattering $\left(L_{3}\right)$. We expect $L_{1}<L_{2}<L_{3}$. Indeed, requirements for dephasing (inelastic scattering) are stronger (weaker) than those for electron heating. Then for the wires with length $L \ll L_{2}$ the Fano factor equals $1 / 3$ and is not affected by inelastic processes; for $L_{2} \ll L \ll L_{3}$ it is above $1 / 3$, and for $L \gg L_{3}$ it goes down and disappears as $L \rightarrow \infty$.

In Section VI we will reconnect to the results presented here within the classical Boltzmann-Langevin approach.

\section{SCATTERING THEORY OF FREQUENCY DEPENDENT NOISE SPECTRA}

\section{A. Introduction. Current conservation}

The investigation of frequency dependent transport, in particular, noise, is important, since it can reveal information about internal energy scales of mesoscopic systems, not available from dc transport. On the other hand, the investigation of the dynamic noise is a more difficult task than the investigation of quasi-static noise. This is true experimentally, since frequency dependent measurements require a particularly careful control of the measurement apparatus (one wants to see the capacitance of the sample and not that of the coaxial cable connecting to the measurement apparatus), and it is also true theoretically. Addressing specifically mesoscopic systems, the conceptual difficulty is that generally it is meaningless to consider the dynamic response of noninteracting electrons. Since this point remains largely unappreciated in the literature, we give here a brief explanation of this statement. Consider the following system of equations of classical electrodynamics ${ }^{3}$,

$$
\begin{aligned}
& \boldsymbol{j}=\boldsymbol{j}_{p}+\frac{1}{4 \pi} \frac{\partial \boldsymbol{E}}{\partial t} ; \quad \boldsymbol{E}=-\nabla \varphi \\
& \nabla^{2} \varphi=-4 \pi \rho \\
& \operatorname{div} \boldsymbol{j}_{p}+\frac{\partial \rho}{\partial t}=0
\end{aligned}
$$

Here $\boldsymbol{j}, \varphi$ and $\rho$ are the density of the electric current (particle current), the electric potential, and the charge density, respectively; $\boldsymbol{E}$ is the electric field. Equation (122) states that the total current $\boldsymbol{j}$ is a sum of the particle current $\boldsymbol{j}_{p}$ and the displacement current, represented by the second term on the rhs. Equations (123) and (124) are the Poisson equation and the continuity equation, respectively; they must be supplemented by appropriate

\footnotetext{
${ }^{33}$ For simplicity, we assume the lattice dielectric constant to be uniform and equal to one.
} 
boundary conditions. We make the following observations.

(i) Equations (122), (123), and (124), taken together, yield div $\boldsymbol{j}=0$ : the total current density has neither sources nor sinks. This is a general statement, which follows entirely from the basic equations of electrodynamics, and has to be fulfilled in any system. Theories which fail to yield a source and sink free total current density cannot be considered as correct. The equation $\operatorname{div} \boldsymbol{j}=0$ is a necessary condition for the current conservation, as it was defined above (Section III).

(ii) The particle current $\boldsymbol{j}_{p}$ is generally not divergenceless, in accordance with the continuity equation (124), and thus, is not necessarily conserved. To avoid a possible misunderstanding, we emphasize that it is not a mere difference in definitions: The experimentally measurable quantity is the total current, and not the particle current. Thus, experimentally, the fact that the particle current is not conserved is irrelevant.

(iii) The Poisson equation (123), representing electronelectron interactions, is crucial to ensure the conservation of the total current. This means that the latter cannot be generally achieved in the free electron model, where the self-consistent potential $\varphi$ is replaced by the external electric potential.

(iv) In the static case the displacement current is zero, and the particle current alone is conserved. In this case the self-consistent potential distribution $\varphi(\boldsymbol{r})$ is generally also different from the external electrostatic potential, but to linear order in the applied voltage the conductance is determined only by the total potential difference. As a consequence of the Einstein relation the detailed spatial variation of the potential in the interior of the sample is irrelevant and has no effect on the total current.

In mesoscopic physics the problem is complicated since a sample is always a part of a larger system. It interacts with the nearby gates (used to define the geometry of the system and to control the number of charges in the system). Thus a complete solution of the above system of equations is usually a hopeless task without some serious approximations. The theoretical task is to choose idealizations and approximations which are compatible with the basic conservation laws expressed by the above equations. For instance, we might want to describe interactions in terms of an effective (screened) interaction instead of the full long range Coulomb interaction. It is then necessary to ensure that such an effective interaction indeed leads to the conservation of current.

Three frequency dependent types of noise spectra should be distinguished: (i) Finite-frequency noise at equilibrium or in the presence of dc voltage. (ii) Zerofrequency noise in the presence of an ac voltage; the resulting spectrum depends on the frequency of the acvoltage. (iii) Finite-frequency noise in the presence of an ac voltage; this quantity depends on two frequencies. Here we are interested mostly in the first type of noise spectra; the second one is only addressed in subsection III . We re-iterate that, generally, one can not find the ac conductance and the current fluctuations from a non-interacting model. Even the finite frequency current-current correlations (noise) at equilibrium or in the presence of a dc voltage source, which are of primary interest in this Section, can not be treated without taking account interactions. A simple way to see this is to note that due to the fluctuation-dissipation theorem, the equilibrium correlation of currents in the leads $\alpha$ and $\beta$ at finite frequency, $S_{\alpha \beta}(\omega)$, is related to the corresponding element of the conductance matrix, $S_{\alpha \beta}(\omega)=2 k_{B} T\left[G_{\alpha \beta}(\omega)+G_{\beta \alpha}^{*}(\omega)\right]$. The latter is a response to average current in the lead $\alpha$ to the ac voltage applied to the lead $\beta$, and is generally interactionsensitive. Thus, calculation of the quantity $S_{\alpha \beta}(\omega)$ also requires a treatment of interactions to ensure current conservation.

We can now be more specific and make the same point by looking at Eq. (51) which represents the fluctuations of the particle current at finite frequency. Indeed, for $\omega=0$ the current conservation $\sum_{\alpha} S_{\alpha \beta}=0$ is guaranteed by the unitarity of the scattering matrix: The matrix $A(\alpha, E, E)$ (Eq. (44)) contains a product of two scattering matrices taken at the same energy, and therefore it obeys the property $\sum_{\alpha} A(\alpha, E, E)=0$. On the other hand, for finite frequency the same matrix $A$ should be evaluated at two different energies $E$ and $E+\hbar \omega$, and contains now a product of two scattering matrices taken at different energies. These scattering matrices generally do not obey the property $\sum_{\alpha} s_{\alpha \beta}^{\dagger}(E) s_{\alpha \gamma}(E+\hbar \omega)=\delta_{\beta \gamma}$, and the current conservation is not fulfilled: $\sum_{\alpha} S_{\alpha \beta}(\omega) \neq 0$.

Physically, this lack of conservation means that there is charge pile-up inside the sample, which gives rise to displacement currents. These displacement currents restore current conservation, and thus need to be taken into account. It is exactly at this stage that a treatment of interactions is required. Some progress in this direction is reviewed in this Section later on.

It is sometimes thought that there are situations when displacement currents are not important. Indeed, the argument goes, there is always a certain energy scale $\hbar \omega_{c}$, which determines the energy dependence of the scattering matrices. This energy scale is set by the level width (tunneling rate) $\Gamma$ for resonant tunnel barriers, the Thouless energy $E_{c}=\hbar D / L^{2}$ for metallic diffusive wires $(D$ and $L$ are the diffusion coefficient and length of the wire, respectively), and the inverse Ehrenfest time (i.e. the time for which an electron loses memory about its initial position in phase space) for chaotic cavities. The scattering matrices may be thought as energy independent for energies below $\hbar \omega_{c}$. Then for frequencies below $\omega_{c}$ we have $\sum_{\alpha} s_{\alpha \beta}^{\dagger}(E) s_{\alpha \gamma}(E+\hbar \omega) \sim \delta_{\beta \gamma}$, and the unitarity of the scattering matrix assures current conservation, $\sum_{\alpha} S_{\alpha \beta}=0$. However, there are time-scales which are not set by the carrier kinetics, like $R C$-times which reflect a collective charge response of the system. In fact, from the few examples for which the ac conductance has been 
examined, we know that it is the collective times which matter ${ }^{34}$. Displacement currents can be neglected only, if we can assure that these collective times are much shorter than any of the kinetic time-scales discussed above. Furthermore, there are problems which can only be treated by taking interactions into account, even at arbitrarily low frequency: Later on in this Section we will discuss the RC-times of mesoscopic conductors capacitively coupled to a gate. The noise induced into a nearby capacitor is proportional to the square of the frequency. Naive discussions which do not consider the energy dependence of the scattering matrix cannot predict such currents.

Let us at first consider the range of frequencies that are much smaller than any inverse kinetic time scale and smaller than any inverse collective response time. This case is in some sense trivial, since the energy dependence of the scattering matrices is neglected, and the system is now not probed on the scale $\omega_{c}$. As a consequence there is no novel information on the system compared to a zero frequency noise measurement. In this case, as we will see, the entire frequency dependence of the noise is due to the frequency dependence of the Fermi functions. However, it is the low-frequency measurements which are more easily carried out, and therefore there is some justification to discuss noise spectra in this frequency interval.

The rest of the Section is organized as follows. Subsection IIIB treats fluctuations of the particle current of independent electrons, either at equilibrium or in the presence of a dc bias, in the regime when the scattering matrices can be assumed to be frequency independent. Subsection III generalizes the same notions for noise caused by an ac bias. Afterwards, we relax the approximation of the energy independence of scattering matrices, and in subsections III D and III E consider two simple examples. In both of them Coulomb interactions prove to be important. Though the theory of ac noise is far from being completed, we hope that these examples, representing the results available by now in the literature, can stimulate further research in this direction.

In this Section, we only review the quantummechanical description of frequency dependent noise, based on the scattering approach. Alternatively, the frequency dependence of shot noise in diffusive conductors may be studied based on the classical BoltzmannLangevin approach 140 145. These developments are described in Section VI.

\footnotetext{
${ }^{34}$ Exceptions are frequency dependent weak localization corrections 137] which depend in addition to the RC-time also on the dwell time 138, and perfect ballistic wires which have a charge neutral mode determined by the transit time as the lowest collective mode 139 .
}

\section{B. Low-frequency noise for independent electrons: at equilibrium and in the presence of dc transport}

General consideration. This subsection is devoted to low-frequency noise, in a regime where the scattering matrices are energy independent. We take the frequency, temperature and the voltage all below $\omega_{c}$, and below any frequencies associated with the collective response of the structure. For simplicity we only consider the two-terminal case, $\mu_{L}=e V, \mu_{R}=0, V \geq 0$. We emphasize again that in this approach the internal energy scales of mesoscopic conductors cannot be probed, nor is there a manifestation of the collective modes. The frequency dependence of noise is entirely due to the Fermi functions.

Our starting point is Eq. (51), which in this form is given in Ref. [146]. Taking the scattering matrices to be energy independent, we write

$$
\begin{aligned}
S(\omega) & \equiv S_{L L}(\omega)=\frac{e^{2}}{2 \pi \hbar}\left\{\sum_{n} T_{n}^{2} \int d E\right. \\
& \times\left[f_{L L}(E, \omega)+f_{R R}(E, \omega)\right]+\sum_{n} T_{n}\left(1-T_{n}\right) \\
& \left.\times \int d E\left[f_{L R}(E, \omega)+f_{R L}(E, \omega)\right]\right\}
\end{aligned}
$$

with the abbreviation

$$
\begin{aligned}
f_{\alpha \beta}(E, \omega) & =f_{\alpha}(E)\left[1-f_{\beta}(E+\hbar \omega)\right] \\
& +\left[1-f_{\alpha}(E)\right] f_{\beta}(E+\hbar \omega) .
\end{aligned}
$$

Here the $T_{n}$ 's are, as before, (energy independent) transmission coefficients. Performing the integration, we obtain

$$
\begin{aligned}
S(\omega) & =\frac{e^{2}}{2 \pi \hbar}\left\{2 \hbar \omega \operatorname{coth}\left(\frac{\hbar \omega}{2 k_{B} T}\right) \sum_{n} T_{n}^{2}\right. \\
& +\left[(\hbar \omega+e V) \operatorname{coth}\left(\frac{\hbar \omega+e V}{2 k_{B} T}\right)\right. \\
& \left.\left.+(\hbar \omega-e V) \operatorname{coth}\left(\frac{\hbar \omega-e V}{2 k_{B} T}\right)\right] \sum_{n} T_{n}\left(1-T_{n}\right)\right\} .
\end{aligned}
$$

This formula expresses the noise spectral power for arbitrary frequencies, voltages, and temperatures (all of them are assumed to be below $\omega_{c}$ ). The frequency dependent functions in Eq. 127) are obtained already in the discussions of noise based on the tunneling Hamiltonian approach for junctions [24] (see also Refs. 226,147,27]). In this approach one expands in the tunneling probability, and consequently, to leading order, terms proportional to $\sum_{n} T_{n}^{2}$ are disregarded. The full expression Eq. (127) including the terms proportional to $T_{n}^{2}$ was derived by Khlus [15] assuming from the outset that the scattering matrix is diagonal. It is a general result for an arbitrary scattering matrix, if the $T_{n}$ 's are taken to be the 
the eigenvalues of $t^{\dagger} t$. Later the result of Khlus was rederived by Yang 148] in connection with the QPC; the many-channel case was discussed by Ueda and Shimizu [149, Liu and Yamamoto [130, and Schoelkopf et al [150.

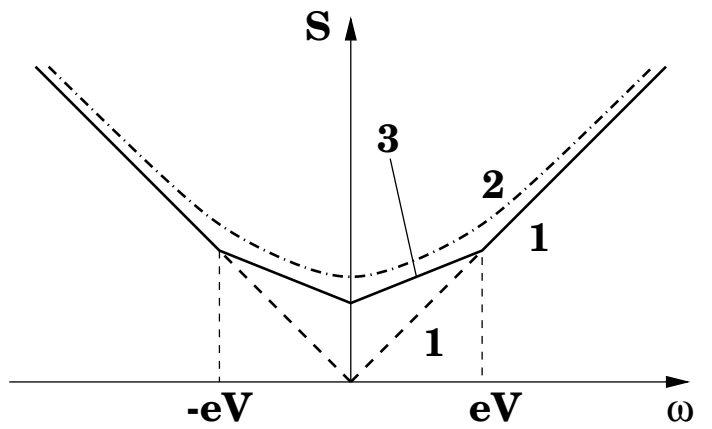

FIG. 22. Frequency dependence of noise for zero temperature, (Eq. (129), solid line), and finite temperatures (dash-dotted line, 2). Line 1 shows equilibrium noise, $S=e^{2}|\omega| \sum T_{n} / \pi$. Line 3 corresponds to the upper line of Eq. (129). In this figure we set $\hbar=1$.

We note first that for $\omega=0$, Eq. (127) reproduces the results for thermal and shot noise presented in Section III (for the two-terminal case and energy independent transmission coefficients). Furthermore, at equilibrium $(V=0)$ it gives the Nyquist noise,

$$
S_{e q}(\omega)=\frac{e^{2} \omega}{\pi} \operatorname{coth}\left(\frac{\hbar \omega}{2 k_{B} T}\right) \sum_{n} T_{n},
$$

as implied by the fluctuation-dissipation theorem. For zero temperature we obtain from Eq. (127)

$$
\begin{gathered}
S(\omega)=\frac{e^{2}}{\pi \hbar} \\
\times\left\{\begin{array}{cc}
\hbar|\omega| \sum_{n} T_{n}^{2}+e V \sum_{n} T_{n}\left(1-T_{n}\right), & \hbar|\omega|<e V \\
\hbar|\omega| \sum_{n} T_{n}, & \hbar|\omega|>e V
\end{array}\right.
\end{gathered}
$$

The frequency dependence is given by a set of straight lines. For zero frequency, the result for shot noise is reproduced, $S=\left(e^{3} V / \pi \hbar\right) \sum_{n} T_{n}\left(1-T_{n}\right)$. At higher frequencies the spectral density increases, and for any finite $\omega$ it is not proportional to the voltage any more. Thus, for finite frequency, like for finite temperature, we do not have pure shot noise. At $\hbar \omega= \pm e V$ the noise spectral power has a discontinuous derivative, and for $|\omega|>e V / \hbar$ the noise spectrum tends to the equilibrium value Eq. (128) determined by the zero-point quantum fluctuations, independent of the voltage. Finite temperature smears the singularities since now the Fermi functions are continuous. The noise spectrum is shown in Fig. 22. It can also be represented differently, if we define excess noise (for $k_{B} T=0$ ) as the difference between the full noise power Eq. (129) and equilibrium noise,
$S_{e x}(\omega)=S(\omega)-e^{2}|\omega| \sum_{n} T_{n} / \pi$. The excess noise is given by

$$
S_{e x}(\omega)=\frac{e^{2}}{\pi \hbar} \sum_{n} T_{n}\left(1-T_{n}\right)(e V-\hbar|\omega|), \quad \hbar|\omega| \leq e V
$$

and zero otherwise.

Eq. (127) is general and valid for all systems under the conditions it was derived. Instead of discussing it for all the examples mentioned in Section III, we consider only the application to a metallic diffusive wire.

Metallic diffusive wires. Performing the disorder averages of transmission coefficients with the distribution function (87), we find the result obtained earlier by Altshuler, Levitov, and Yakovets [76,

$$
\begin{aligned}
S(\omega) & =\frac{1}{3} G\left\{4 \hbar \omega \operatorname{coth}\left(\frac{\hbar \omega}{2 k_{B} T}\right)\right. \\
& +(\hbar \omega+e V) \operatorname{coth}\left(\frac{\hbar \omega+e V}{2 k_{B} T}\right) \\
& \left.+(\hbar \omega-e V) \operatorname{coth}\left(\frac{\hbar \omega-e V}{2 k_{B} T}\right)\right\},
\end{aligned}
$$

where $G=e^{2} N_{\perp} l / 2 \pi \hbar L$ is the conductance of a wire with mean free path $l$ and length $L$.

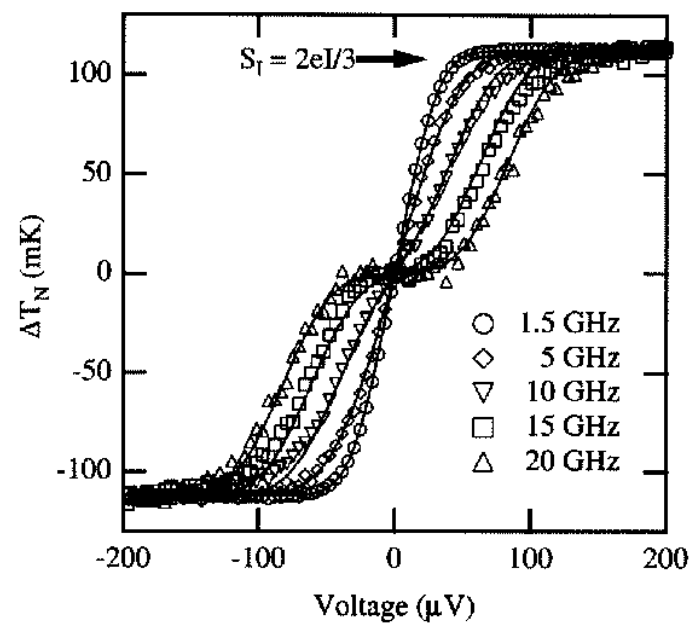

FIG. 23. Experimental results of Schoelkopf et al 150] for the frequency dependence of noise in metallic diffusive wires. Solid lines for each frequency indicate the theoretical result (130). The quantity shown on the vertical axis is essentially $\partial S / \partial V$.

An experimental investigation of the frequency dependent noise in diffusive gold wires is presented by Schoelkopf et al $\mid 150$; this paper reports one of the only two presently available measurements of ac noise. They find a good agreement with Eq. (130), where the electron temperature $T$ was used as a fitting parameter 35

\footnotetext{
${ }^{35}$ Due to effects of electron heating; see Section V1.
} 
(the same for all frequencies). The results of Ref. 150 are plotted in Fig. 23 as a function of voltage for different frequencies; theoretical curves are shown as solid lines. In the experiment, the highest frequency corresponded approximately to the Thouless energy, and in this regime Eq. (130) is well justified.

We have emphasized earlier that in this frequency regime no internal dynamics of the system is probed. The fact that Eq. (130) agrees with experiment is a consequence of the strong screening in metals. In poor metals, the RC-times might become long. A finite value of the screening length may permit charge fluctuations and consequently modify the noise behavior even at relative low frequencies. We address this issue in Section V1.

Inelastic scattering. Now one can ask: What is the effect of inelastic scattering on frequency dependent noise in the regime, where the scattering matrices can be taken to be energy independent. This problem was studied by Ueda and Shimizu [149], and later by Zheng, Wang, and Liu [151], who included electron-phonon interaction directly into the scattering approach, and by Liu and Yamamoto [130], who used an approach based on dephasing voltage probes. The general conclusion is, that like in the case of the zero frequency, inelastic scattering suppresses noise 36 .

\section{Low-frequency noise for independent electrons: Photon-assisted transport}

Now we generalize the results of the preceding subsection to the case when the applied voltage is timedependent; the scattering matrices are still assumed not to depend on energy. The fluctuations in the presence of a potential generated by an ac magnetic flux were treated by Lesovik and Levitov [152]; the fluctuation spectrum in the presence of oscillating voltages applied to the contacts of the sample was obtained in Ref. 153]. The results are essentially the same; below we follow the derivation of Ref. 1153.

We consider a two-terminal conductor; the chemical potential of the right reservoir is kept fixed (we assume it to be zero), while the left reservoir is subject to a constant voltage $\bar{V}$ plus the oscillating component $U(t)=V(\Omega) \cos \Omega t$. One cannot simply use the stationary scattering theory as described in Section II. Instead, the scattering states in the left lead are now solutions of the time-dependent Schrödinger equation,

$$
\begin{aligned}
\psi_{n}(\mathbf{r}, E, t) & =\chi_{L n}\left(\boldsymbol{r}_{\perp}\right) e^{i k_{L n} z-i E t / \hbar} \\
& \times \sum_{l=-\infty}^{\infty} J_{l}\left(\frac{e V(\Omega)}{\hbar \Omega}\right) e^{-i l \Omega t}
\end{aligned}
$$

\footnotetext{
${ }^{36}$ In contrast, the effect of dephasing on the finite frequency noise seems not to have been investigated.
}

Thus, in the presence of an oscillating voltage each state with the central energy $E$ is split to infinitely many subbands with energies $E+l \hbar \Omega$, which have smaller spectral weight. Following the literature on tunneling, this phenomenon is called photon-assisted transport, since electrons with higher energies $(l>0)$ have higher transmission probabilities, and might propagate through the sample thanks to the additional energy.

Now we use a formal trick, assuming that the oscillating potential only exists asymptotically far from the sample (and there Eq. (131) is valid), and decays slowly towards the sample. Thus, there is a certain portion of the left lead, where there is no oscillating potential, but still no scattering. The annihilation operators in this part of the left lead have thus the form

$$
\hat{a}_{L n}(E)=\sum_{l} J_{l}\left(\frac{e V(\Omega)}{\hbar \Omega}\right) \hat{a}_{L n}^{\prime}(E-l \hbar \Omega),
$$

where the operators $\hat{a}^{\prime}$ describe the states of the left reservoir. Instead of Eq. (34), we obtain

$$
\begin{aligned}
\hat{I}_{L}(t) & =\frac{e}{2 \pi \hbar} \sum_{\alpha \beta} \sum_{m n} \int d E d E^{\prime} e^{i\left(E-E^{\prime}\right) t / \hbar} \\
& \times \sum_{l k} J_{l}\left(\frac{e V_{\alpha}}{\hbar \Omega}\right) J_{k}\left(\frac{e V_{\beta}}{\hbar \Omega}\right) \\
& \times \hat{a}_{\alpha m}^{\prime \dagger}(E-l \hbar \Omega) A_{\alpha \beta}^{m n}\left(L ; E, E^{\prime}\right) \hat{a}_{\beta n}^{\prime}\left(E^{\prime}-k \hbar \Omega\right)
\end{aligned}
$$

and we set $V_{L}=V(\Omega), V_{R}=0$. Finally, we assume that the frequency is not too high, so that the left reservoir can be considered to be at (dynamic) equilibrium at any instant of time. Then the averages of the operators $\hat{a}^{\prime}$ are essentially equilibrium averages, expressed through the Fermi functions, $f_{L}=f_{F}(E-e \bar{V})$ and $f_{R}=f_{F}(E)$.

In the presence of a time-dependent voltage, the correlation function $S_{L L}(49)$ depends not only on the time difference $t-t^{\prime}$, but also on the absolute time $\tau=\left(t+t^{\prime}\right) / 2$. In the following, we are interested in the noise spectra on a time scale long compared to $\Omega^{-1}$. Then the noise power can be averaged over $\tau$,

$$
S\left(t-t^{\prime}\right)=\frac{1}{\tilde{\tau}} \int_{0}^{\tilde{\tau}} d \tau S\left(t-t^{\prime}, \tau\right), \quad \tilde{\tau}=\frac{2 \pi}{\Omega} .
$$

Leaving more general cases aside, we only give an expression for the zero-frequency component of $S_{L L}$ 152,153,

$$
\begin{aligned}
S_{L L}(\omega=0, \Omega) & =\frac{e^{2}}{2 \pi \hbar} \sum_{\alpha \beta} \int d E \sum_{l} J_{l}^{2}\left(\frac{e\left(V_{\alpha}-V_{\beta}\right)}{\hbar \Omega}\right) \\
& \times \operatorname{Tr}\left[A_{\alpha \beta}(L) A_{\beta \alpha}(L)\right] f_{\alpha \beta}(E, l \hbar \Omega),
\end{aligned}
$$

where the matrices $A$ are explicitly assumed to be energy independent. For zero external frequency, $\Omega=0$, only the Bessel function with $l=0$ survives, and should be taken equal to one for any $\alpha$ and $\beta$; then we reproduce the zero-frequency expression (52). Performing the energy 
integration and introducing the transmission coefficients, we obtain 154

$$
\begin{aligned}
S(\Omega) & =\frac{e^{2}}{2 \pi \hbar}\left\{4 k_{B} T \sum_{n} T_{n}^{2}\right. \\
& =\sum_{l=-\infty}^{\infty} J_{l}^{2}\left(\frac{e V(\Omega)}{\hbar \Omega}\right) \sum_{n} T_{n}\left(1-T_{n}\right) \\
& \times\left[(l \hbar \Omega+e \bar{V}) \operatorname{coth}\left(\frac{l \hbar \Omega+e \bar{V}}{2 k_{B} T}\right)\right. \\
& \left.\left.+(l \hbar \Omega-e \bar{V}) \operatorname{coth}\left(\frac{l \hbar \Omega-e \bar{V}}{2 k_{B} T}\right)\right]\right\} .
\end{aligned}
$$

For zero temperature, Eq. (134) exhibits singularities at voltages $\bar{V}=l \hbar \Omega / e$ : The derivative $\partial S / \partial \bar{V}$ is a set of steps. The height of each step depends on the ac voltage due to the Bessel function in Eq. (134).

Lesovik and Levitov [152] considered a geometry of (an almost closed) one-channel loop of length L connected to two reservoirs. The loop contains a scatterer, and is pierced by the time-dependent magnetic flux $\Phi(t)=$ $\Phi_{a} \sin \Omega t$. The time dependent flux generates an electric field and thus an internal voltage $U(t)=U_{a} \cos \Omega t$ with $e U_{a}=2 \pi\left(\Phi_{a} / \Phi_{0}\right)(L / 2 \pi R) \hbar \Omega$, where $L$ is the length of the segment on the circle with radius $R$. In addition, a constant voltage $\bar{U}$ is applied. The magnetic flux can be incorporated in the phase of the scattering matrix, and the previous analysis is easily generalized for this case. Ref. [152] found that for zero temperature $\partial S / \partial \bar{U}$ is again a step function of voltage. Steps occur at $\bar{U}=l \hbar \Omega / e$, and the height of each step is $\lambda_{l}=T(1-T) J_{l}^{2}\left(e U_{a} / \hbar \Omega\right)$, where $T$ is the transmission coefficient. In the Ref. 152 the argument of the Bessel function is written in terms of the ratio of fluxes $2 \pi \Phi_{a} / \Phi_{0}$ and the effect is called a non-stationary Aharonov-Bohm effect. However, we emphasize that what is investigated is the response to the external electric field generated by the oscillating flux. This is a classical response, unrelated to any AharonovBohm type effect.

It is remarkable that in the case of energy independent transmission probabilities the response to the electric field considered in Ref. [152] is the same as that of an oscillating voltage $V \equiv U_{a}$ applied to a contact.

Levinson and Wölfle [155] considered a related problem: the noise for the transmission through a barrier with an oscillating random profile (originating, for instance, from the external irradiation). The latter is represented by a one-dimensional potential

$$
U(x, t)=U_{0}(x)+\delta U(x, t) .
$$

The random component $\delta U$ is assumed to be zero on average, and its second moment is a function of $t-t^{\prime}$. In this case, current-current correlations, $\left\langle\delta I(t) \delta I\left(t^{\prime}\right)\right\rangle$, for each particular realization of the random potential depend on both times $t$ and $t^{\prime}$. However, after averaging over disorder realizations, the resulting noise only depends on $t-t^{\prime}$ and can be Fourier transformed. The scattering matrices are energy independent for frequencies below the inverse time of flight through the barrier, $\omega \ll v_{F} / L$, with $L$ being the length of the barrier.

A remarkable feature of this model is that if the barrier and the irradiation are symmetric, $U_{0}(x)=U_{0}(-x)$, and $\delta U(x, t)=\delta U(-x, t)$, and no voltage is applied between the reservoirs, there is no current generated by the irradiation. On the other hand, a non-equilibrium contribution to noise exists. In particular, when the second moment of the random potential is $\overline{\delta U(x, t) \delta U\left(x^{\prime}, t^{\prime}\right)}=V_{0}^{2} \delta\left(x-x^{\prime}\right)$, it happens to have the same frequency structure as the equilibrium one (128), but with the coefficient proportional to $V_{0}^{2}$. The voltage applied to the reservoirs is, as usual, one more source of non-equilibrium noise. For further details, we refer the reader to Ref. 155].

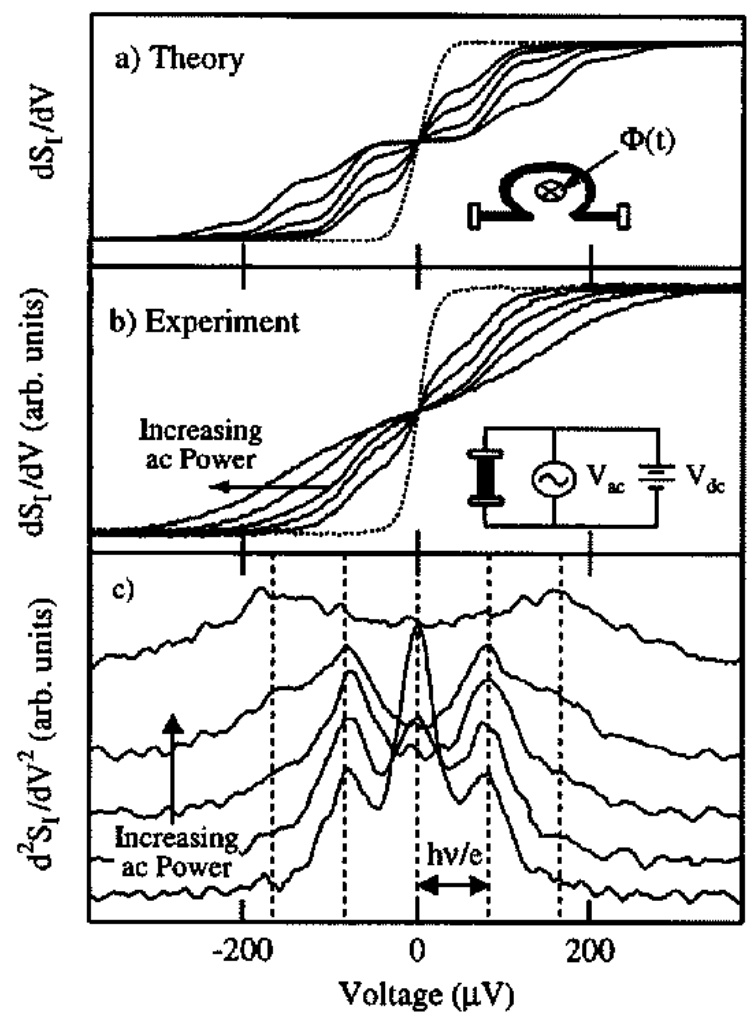

FIG. 24. (a) Theoretical results (134) for various amplitudes of the ac voltage; dotted line shows the dc results; (b) Experimental results of Schoelkopf et al 154 for the same parameters; (c) Experimental results plotted as $\partial^{2} S / \partial \bar{V}^{2}$ for different ac voltage amplitudes. The frequency $\Omega$ is fixed.

Experimentally, noise in response to a simultaneous $\mathrm{dc}$ voltage and ac voltage applied to the contacts of the sample was studied by Schoelkopf et al [154] in phase coherent metallic diffusive wires 3 . They measured zero-

${ }^{37}$ As usual, for metallic diffusive wires $\sum_{n} T_{n}^{2}$ and 
frequency noise as a function of voltage $\bar{V}$ in the $\mathrm{GHz}$ range. The results are presented in Fig. 24 as $\partial S / \partial \bar{V}$ and $\partial^{2} S / \partial \bar{V}^{2}$. The latter quantity is expected to have sharp peaks at the resonant voltages $\bar{V}=l \hbar \Omega / e$. Indeed, three peaks, corresponding to $l=0, \pm 1$ are clearly seen; others are smeared by temperature and not so well pronounced.

Ref. 153] emphasized the need for a self-consistent calculation of photon-assisted transport processes even in the case that the only quantity of interest are the currents or noise-spectra measured at zero frequency. The true electric field in the interior of the conductor is not the external field. The fact that the experimental results agree rather well with the simple results presented here (which do not invoke any self-consistency) is probably a consequence of the effective screening of the metallic conductor. The true potential is simply linear in the range of frequencies investigated experimentally. A self-consistent spectrum for photon-assisted noise spectra can probably be developed along the lines of Ref. 153].

\section{Noise of a capacitor}

Now we turn to the problems where the energy dependence of the scattering matrices is essential. Rather than trying to give the general solution (which is only available for the case when the potential inside the system is spatially uniform [156, 157]), we provide a number of examples which could serve as a basis for further investigations of finite frequency noise.

The simple case of shot noise in a ballistic wire was studied by Kuhn, Reggiani, and Varani 158,159. They found signatures of the inverse flight time $v_{F} / L$. However, the interactions are taken into account only implicitly via what the authors call "quantum generalization of the Ramo-Shockley theorem". We do not know in which situations this approach is correct, and it certainly cannot be correct universally. Thus, even this simple case cannot be considered as solved and needs further consideration.

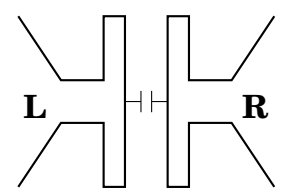

a)

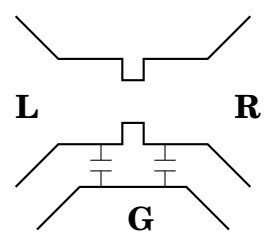

b)
FIG. 25. A mesoscopic capacitor (a); a mesoscopic conductor vis-a-vis a gate (b).

$\sum_{n} T_{n}\left(1-T_{n}\right)$ must be replaced by $2 l N_{\perp} / 3 L$ and $l N_{\perp} / 3 L$, respectively.
We start from the simplest system - a mesoscopic capacitor (Fig. 25a), which is connected via two leads to equilibrium reservoirs. Instead of the full Poisson equation interactions are described with the help of a geometrical capacitance $C$. There is no transmission from the left to the right plate, and therefore there is no dc current from one reservoir to the other. Moreover, this system does not exhibit any noise even at finite frequency if the scattering matrix is energy independent. Indeed, if only the matrices $s_{L L}$ and $s_{R R}$ are nonzero, we obtain from Eq. (51)

$$
\begin{aligned}
S_{\alpha \beta}^{(0)}(\omega) & =\frac{e^{2}}{2 \pi \hbar} \delta_{\alpha \beta} \int d E \operatorname{Tr}\left\{\left[1-s_{\alpha \alpha}^{\dagger}(E) s_{\alpha \alpha}(E+\hbar \omega)\right]\right. \\
& \left.\times\left[1-s_{\alpha \alpha}^{\dagger}(E+\hbar \omega) s_{\alpha \alpha}(E)\right]\right\} \\
& \times f_{\alpha}(E)\left(1-f_{\alpha}(E+\hbar \omega)\right) .
\end{aligned}
$$

Here we used the superscript (0) to indicate that the fluctuations of the particle current, and not the total current, are discussed. If the scattering matrices are energy independent, Eq. (135) is identically zero due to unitarity. Another way to make the same point is to note that since $S_{L R}=0$, the only way to conserve current would be $S_{L L}=0$.

Before proceeding to solve this problem, we remark that Eq. (135) describes an equilibrium fluctuation spectrum and via the fluctuation dissipation theorem $S_{\alpha \alpha}^{(0)}(\omega)=2 \hbar \omega g_{\alpha \alpha}(\omega) \operatorname{coth}\left(\hbar \omega / 2 k_{B} T\right)$ is related to the real part of a conductance $g_{\alpha \alpha}(\omega)$ given by

$$
\begin{aligned}
g_{\alpha \alpha}(\omega) & =\frac{e^{2}}{\hbar} \int d E \operatorname{Tr}\left\{\left[1-s_{\alpha \alpha}^{\dagger}(E) s_{\alpha \alpha}(E+\hbar \omega)\right]\right\} \\
& \times \frac{f_{\alpha}(E)-f_{\alpha}(E+\hbar \omega)}{\hbar \omega} .
\end{aligned}
$$

In what follows, the fluctuation dissipation theorem for the non-interacting system also ensures this theorem for the interacting system.

Current conservation is restored only if interactions are taken into account. Below we assume that charging effects are the only manifestation of interactions [157, 131]. We first present the general result which is, within the limitations stated above, valid for arbitrary frequencies. We then consider in detail the low frequency expansion of this result which can be expressed in physically appealing quantities: an electrochemical capacitance and a charge relaxation resistance.

General result. Our starting point are the particle current operators in the left and right lead $(43), \hat{I}_{L}^{(0)}(t)$ and $\hat{I}_{R}^{(0)}(t)$. Their fluctuation spectra are determined by Eq. (135). Now we must take into account that the total currents are in fact not just the particle currents but contain an additional contribution generated by the fluctuating electrostatic potential on the capacitor plates. We introduce the operators of the potential on the left $\hat{u}_{L}(t)$ and right $\hat{u}_{R}(t)$ plate. The fluctuation of the total current through the lead $\alpha$ can be written in operator form as follows, 


$$
\Delta \hat{I}_{\alpha}(t)=\delta \hat{I}_{\alpha}(t)+\int d t^{\prime} \chi_{\alpha}\left(t-t^{\prime}\right) \delta \hat{u}_{\alpha}\left(t^{\prime}\right), \quad \alpha=L, R
$$

Here we introduced $\delta \hat{I}_{\alpha}(t)=\hat{I}_{\alpha}^{(0)}(t)-\left\langle I_{\alpha}^{(0)}\right\rangle$ and $\delta \hat{u}_{\alpha}(t)=$ $\hat{u}_{\alpha}(t)-\left\langle u_{\alpha}\right\rangle$. Furthermore, $\chi_{\alpha}$ is the response function which determines the current generated at contact $\alpha$ in response to an oscillating potential on the capacitor plate. For the simple case considered here, it can be shown 157 that this response function is directly related to the the ac conductance, Eq. (136), for noninteracting electrons, which gives the current through the lead $\alpha$ in response to a voltage applied to the same lead, $\chi_{\alpha}(\omega)=-g_{\alpha \alpha}(\omega)$. The minus sign is explained by noting that the current fluctuation is the response to $\mu_{\alpha}-u_{\alpha}$ rather than $u_{\alpha}$. The operators $\Delta \hat{I}$, and not $\delta \hat{I}$, determine the experimentally measured quantities.

The total current in this system is the displacement current. In the capacitance model the charge of the capacitor $\hat{Q}$ is given by $\hat{Q}=C\left(\hat{u}_{L}-\hat{u}_{R}\right)$. Note that this is just the Poisson equation expressed with the help of a geometrical capacitance. To the extent that the potential on the capacitor plate can be described by a uniform potential this equation is valid for all frequencies. Then the fluctuations of the current through the left and right leads are

$$
\Delta \hat{I}_{L}(t)=\frac{\partial}{\partial t} \delta \hat{Q}(t)=C \frac{\partial}{\partial t}\left[\delta \hat{u}_{L}(t)-\delta \hat{u}_{R}(t)\right],
$$

and $\Delta \hat{I}_{L}(t)=-\Delta \hat{I}_{R}(t)$. Thus, the conservation of the total current is assured. In contrast to the non-interacting problem, the currents to the left and right are now completely correlated. Equations (137) and (138) can be used to eliminate the voltage fluctuations. The result is conveniently expressed in the frequency representation,

$$
\begin{aligned}
\Delta \hat{I}_{L}=-\Delta \hat{I}_{R} & =\frac{i \omega C}{g_{L L} g_{R R}-i \omega C\left(g_{L L}+g_{R R}\right)} \\
& \times\left[g_{L L} \delta \hat{I}_{R}-g_{R R} \delta \hat{I}_{L}\right] .
\end{aligned}
$$

The noise power $S \equiv S_{L L}$ becomes

$$
\begin{aligned}
S(\omega) & =\frac{\omega^{2} C^{2}}{\left|g_{L L} g_{R R}-i \omega C\left(g_{L L}+g_{R R}\right)\right|^{2}} \\
& \times\left[\left|g_{R R}\right|^{2} S_{L L}^{(0)}+\left|g_{L L}\right|^{2} S_{R R}^{(0)}\right] .
\end{aligned}
$$

Eq. (139) expresses the frequency dependent noise spectrum of the interacting system in terms of the conductances $g_{L L}(\omega), g_{R R}(\omega)$ and the noise spectra $S_{L L}^{(0)}, S_{R R}^{(0)}$ of the problem without interactions. Together with Eq. (135), this is the result of the first step of our calculation. Note that the noise of the capacitor depends only implicitly, through the scattering matrices and the Fermi functions, on the stationary (dc) voltage difference across the capacitor. Eq. (139) is thus valid independently on whether the potentials in the two reservoirs are the same or not.

So far, our consideration is valid in the entire frequency range up to the frequencies at which the concept of a single potential no longer holds: $\omega \ll e^{2} / \epsilon d$, where $\epsilon$ and $d$ are the static susceptibility and the distance between the capacitor plates, respectively.

Low-frequency expansion. Now we turn to the lowfrequency expansion of Eq. (139), leaving only the leading term. The expansion of $g_{\alpha \alpha}$ can be easily obtained 157,

$$
\begin{aligned}
g_{\alpha \alpha}(\omega) & =-i \omega e^{2} \nu_{\alpha} \mathcal{A}_{\alpha}+O\left(\omega^{2}\right), \\
\nu_{\alpha} \mathcal{A}_{\alpha} & =-\frac{1}{2 \pi i} \int d E \frac{\partial f_{\alpha}}{\partial E} \operatorname{Tr}\left(s_{\alpha \alpha}^{\dagger}(E) \frac{\partial s_{\alpha \alpha}(E)}{\partial E}\right),
\end{aligned}
$$

where $\nu_{\alpha}$ is the density of states per unit area and $\mathcal{A}_{\alpha}$ is the area of the cross-section of the plate $\alpha$. The fact that the density of states can be expressed in terms of the scattering matrix is well known [160,161. Expanding Eq. (135), we write

$$
\begin{aligned}
S_{\alpha \alpha}^{(0)} & =\frac{e^{2} \hbar \omega^{2} k_{B} T}{\pi} \int d E\left(-\frac{\partial f_{\alpha}}{\partial E}\right) \operatorname{Tr}\left(s_{\alpha \alpha}^{\dagger}(E) \frac{\partial s_{\alpha \alpha}(E)}{\partial E}\right)^{2} \\
& +O\left(|\omega|^{3}\right) .
\end{aligned}
$$

Now we introduce the electrochemical capacitance,

$$
C_{\mu}^{-1} \equiv C^{-1}+\left(e^{2} \nu_{L} \mathcal{A}_{L}\right)^{-1}+\left(e^{2} \nu_{R} \mathcal{A}_{R}\right)^{-1},
$$

and the charge relaxation resistances,

$$
\begin{aligned}
R_{q \alpha} & =\frac{\hbar}{4 \pi e^{2} \nu_{\alpha}^{2} \mathcal{A}_{\alpha}^{2}} \int d E\left(-\frac{\partial f_{\alpha}}{\partial E}\right) \\
& \times \operatorname{Tr}\left(s_{\alpha \alpha}^{\dagger}(E) \frac{\partial s_{\alpha \alpha}(E)}{\partial E}\right)^{2} .
\end{aligned}
$$

These two quantities, which determine the RC-time of the mesoscopic structure, now completely specify the low frequency noise of the capacitor. A little algebra gives 157, 131

$$
S=4 k_{B} T \omega^{2} C_{\mu}^{2}\left(R_{q L}+R_{q R}\right)+O\left(\omega^{3}\right) .
$$

We remark that the charge relaxation resistance is determined by half the resistance quantum $\pi \hbar / e^{2}$ and not $2 \pi \hbar / e^{2}$, reflecting the fact that each plate of the capacitor is coupled to one reservoir only.

We note now that the low-frequency expansion of the ac conductance (admittance) of the same capacitor 157] has a form

\footnotetext{
${ }^{38}$ For zero temperature, the expansion of Eq. 135 starts with a term proportional to $|\omega|^{3}$ rather than $\omega^{2}$. As a result, $4 k_{B} T$ is replaced by $2 \hbar|\omega|$ in the final expression (143) for noise $S$.
} 


$$
G(\omega)=-i \omega C_{\mu}+\omega^{2} C_{\mu}^{2}\left(R_{q L}+R_{q R}\right)+O\left(\omega^{3}\right) .
$$

Thus, we see that the fluctuation-dissipation theorem is also obeyed for the interacting system. Again, the noise spectrum of this equilibrium system contains the same information as the admittance. We emphasize once more that it is not required that the electrochemical potential of the two plates of the capacitor are identical. The results given above also hold if there is a large dc voltage applied across the capacitor.

The electrochemical capacitance and the charge relaxation resistance have been evaluated for a number of examples. In the limit of one quantum channel only, the charge relaxation resistance is universal, independent of the properties of the scattering matrix, and given by $R_{q}=\pi \hbar / e^{2}$. This is astonishing in view of the fact that if a tunnel barrier is inserted in the channel connecting the capacitor plate to the reservoir one would expect a charge relaxation resistance that diverges as the tunnel barrier becomes more and more opaque. For a chaotic cavity connected via a perfect single-channel lead to a reservoir and coupled capacitively to a macroscopic gate, the distribution of the electrochemical capacitance has been given in Ref. 162]. In this case the charge relaxation resistance, as mentioned above, is universal and given by $R_{q}=\pi \hbar / e^{2}$. For a chaotic cavity coupled via an $N$-channel quantum point contact to a reservoir and capacitively coupled to a macroscopic gate, the capacitance and charge relaxation resistance can be obtained from the results of Brouwer and one of the authors [138]. For large $N$, for an ensemble of chaotic cavities, the capacitance fluctuations are very small, and the averaged charge relaxation resistance is given by $R_{q}=2 \pi \hbar / e^{2} N$. If a tunnel barrier is inserted into the contact, the ensemble averaged resistance is 163 $R_{q}=2 \pi \hbar / e^{2} T N$ for a barrier which couples each state inside the cavity with transmission probability $T$ to the reservoir. In accordance with our expectation the charge relaxation resistance is determined by the two-terminal tunnel barrier resistance $R_{t}=2 \pi \hbar / e^{2} T N$. For additional examples we refer the reader to Ref. [164] which presents an overview of the known charge relaxation resistances $R_{q}$ for mesoscopic conductors.

\section{E. Shot noise of a conductor observed at a gate}

It is interesting to ask what would be measured at a gate that couples capacitively to a conductor which is in a transport state. In such a situation, in the zerotemperature limit, the low frequency noise in the conductor is the shot noise discussed in this Review. Thus we can ask, what are the current fluctuations capacitively induced into a gate due to the shot noise in the nearby conductor? To answer this question we consider a mesoscopic conductor vis-a-vis a macroscopic gate [98]. The whole system is considered as a three-terminal structure, with $L$ and $R$ labeling the contacts of the conductor, and
$G$ denoting the gate (Fig. 25b). The gate and the conductor are coupled capacitively with a geometrical capacitance $C$. For a macroscopic gate the fluctuations of the potential within the gate are small and can be neglected. Finally, the most crucial assumption is that the potential inside the mesoscopic conductor is uniform and may be described by a single (fluctuating) value $u$. This assumption is often made in the discussion of the Coulomb blockade effect, but it is in reality almost never satisfied $^{39}$.

We provide a solution to this problem by extending the discussion of the mesoscopic capacitor. We start from the operators of the particle currents, $\hat{I}_{\alpha}^{(0)}$. We have $\hat{I}_{G}^{(0)}=0$, since without interactions there is no current through a macroscopic gate, and correlations of other current operators are given by Eq. (51). We introduce the operator of potential fluctuation $\delta \hat{u}(t)$ in the conductor; the charge fluctuation is $\delta \hat{Q}(t)=C \delta \hat{u}(t)$. Since there is transmission from the left to the right, we write for the fluctuations of the total currents,

$$
\begin{aligned}
& \Delta \hat{I}_{\alpha}(t)=\delta \hat{I}_{\alpha}(t)+\int_{-\infty}^{t} d t^{\prime} \chi_{\alpha}\left(t-t^{\prime}\right) \delta \hat{u}\left(t^{\prime}\right), \quad \alpha=L, R \\
& \Delta \hat{I}_{G}(t)=-C \frac{\partial}{\partial t} \delta \hat{u}(t) .
\end{aligned}
$$

with the condition $\Delta \hat{I}_{L}^{(0)}+\Delta \hat{I}_{R}^{(0)}=C \partial \delta \hat{u}(t) / \partial t$, which ensures the current conservation. It is important that the quantities $\chi_{\alpha}$, which determine the response of currents at the terminals to the potential inside the sample, must be evaluated at equilibrium, and, since this potential is time dependent, the ac current should be taken. The entire dependence on $u$ of the average current is due to scattering matrices. In the semi-classical approximation they depend on the combination $E-e u$, and the derivative with respect to the internal potential is essentially the derivative with respect to energy. Retaining only the leading order in frequency, we obtain (see e.g. 136])

$$
\chi_{\alpha}(\omega)=i \omega e^{2} N_{\alpha}+O\left(\omega^{2}\right),
$$

\footnotetext{
${ }^{39}$ There are no quantum-mechanical calculations of frequency dependent noise with the potential profile taken into account available in the literature. However, when the conductor is a perfect wire, with a nearby gate, the existing calculation of ac conductance 165 can be generalized to calculate noise. One has to start from the field operators, write a density operator, and solve the Poisson equation as an operator equation for the field operator of the (electro-chemical) potential. This lengthy calculation leads to an obvious result: there is no non-equilibrium noise in the absence of backscattering. Though this outcome is trivial, we hope that the same approach may serve as a starting point to solve other problems, like a wire with backscattering. A related discussion was developed classically for the frequency dependent noise in diffusive conductors 140 145, see Section VI.
} 


$$
\begin{aligned}
N_{\alpha} & =-\frac{1}{4 \pi i} \int d E \frac{\partial f}{\partial E} \sum_{\beta} \\
& \times \operatorname{Tr}\left(s_{\alpha \beta}^{\dagger} \frac{\partial s_{\alpha \beta}}{\partial E}-\frac{\partial s_{\alpha \beta}^{\dagger}}{\partial E} s_{\alpha \beta}(E)\right),
\end{aligned}
$$

which is the analog of Eq. (140) for a multi-probe conductor. The quantities $N_{\alpha}$, called emittances in Ref. [136], obey the rule $N_{L}+N_{R}=\nu \mathcal{A}$ (with $\nu$ and $\mathcal{A}$ being the density of states and the area/volume of the conductor, respectively). They have the meaning of a density of the scattering states which describes the electrons exiting eventually through the contact $\alpha$, irrespectively of the contact they entered through.

Combining Eqs. (145) and (146), we obtain

$$
\begin{aligned}
& \Delta \hat{I}_{L}=\left[1-e^{2} N_{L} K\right] \delta \hat{I}_{L}-e^{2} N_{L} K \delta \hat{I}_{L}, \\
& \Delta \hat{I}_{R}=-e^{2} N_{R} K \delta \hat{I}_{L}+\left[1-e^{2} N_{R} K\right] \delta \hat{I}_{R}, \\
& \Delta \hat{I}_{G}=-C K\left[\delta \hat{I}_{L}+\delta \hat{I}_{R}\right]
\end{aligned}
$$

where $K \equiv\left(C+e^{2} N_{L}+e^{2} N_{R}\right)^{-1}$ plays the role of an effective interaction which determines the change in the electrostatic potential inside the conductor in response to a variation of the charge inside the conductor. In the following, we are only interested in the fluctuations of the current through the gate. For this quantity, which vanishes for zero frequency, we obtain

$$
S_{G G}=C^{2} K^{2}\left[S_{L L}^{(0)}+S_{L R}^{(0)}+S_{R L}^{(0)}+S_{R R}^{(0)}\right] .
$$

We note that the sum of all the current fluctuation spectra in Eq. (147) is just the fluctuation spectrum of the total charge on the conductor: The continuity equation gives [98] $\sum_{\alpha} \hat{I}_{\alpha}(\omega)=i \omega e \hat{\mathcal{N}}(\omega)$ where $\hat{\mathcal{N}}$ is the operator of the charge in the mesoscopic conductor. From the current operator Eq. (43) we obtain

$$
\begin{aligned}
\hat{\mathcal{N}}(t) & =\frac{e}{2 \pi \hbar} \sum_{\alpha \beta \gamma} \sum_{m n} \int d E d E^{\prime} e^{i\left(E-E^{\prime}\right) t / \hbar} \\
& \times \hat{a}_{\beta m}^{\dagger}(E) \mathcal{N}_{\beta \gamma}^{m n}\left(E, E^{\prime}\right) \hat{a}_{\gamma n}\left(E^{\prime}\right),
\end{aligned}
$$

with the non-diagonal density of states elements $\mathcal{N}_{\beta \gamma}^{m n}$

$$
\begin{aligned}
\mathcal{N}_{\beta \gamma}^{m n}\left(E, E^{\prime}\right) & =\frac{1}{2 \pi i\left(E-E^{\prime}\right)} \sum_{\alpha}\left[\delta_{m n} \delta_{\alpha \beta} \delta_{\alpha \gamma}\right. \\
& \left.-\sum_{k} s_{\alpha \beta ; m k}^{\dagger}(E) s_{\alpha \gamma ; k n}\left(E^{\prime}\right)\right]
\end{aligned}
$$

or in matrix notation $\mathcal{N}_{\beta \gamma}=(1 / 2 \pi i \omega) \sum_{\alpha} A_{\beta \gamma}(\alpha, E, E+$ $\hbar \omega)$ with the current matrix $A_{\beta \gamma}\left(\alpha, E, E^{\prime}\right)$ given by Eq. (44). Thus, instead of Eq. (147) we can also express the fluctuation spectrum of the current at the gate in terms of the charge fluctuation spectrum

$$
S_{G G}=e^{2} C^{2} K^{2} \omega^{2} S_{N N}^{(0)}
$$

with

$$
\begin{aligned}
S_{N N}^{(0)}(\omega) & =\frac{e^{2}}{2 \pi \hbar} \sum_{\gamma \delta} \sum_{m n} \int d E \mathcal{N}_{\gamma \delta}^{m n}(E, E+\hbar \omega) \\
& \times \mathcal{N}_{\delta \gamma}^{n m}(E+\hbar \omega, E)\left\{f_{\gamma}(E)\left[1-f_{\delta}(E+\hbar \omega)\right]\right. \\
& \left.+\left[1-f_{\gamma}(E)\right] f_{\delta}(E+\hbar \omega)\right\} .
\end{aligned}
$$

Eq. (151) is, in the absence of interactions, the general fluctuation spectrum of the charge on a mesoscopic conductor.

In the zero-temperature limit, we obtain 98]

$$
\begin{aligned}
& S_{G G} \\
= & 2 C_{\mu}^{2} \omega^{2}\left\{\begin{array}{cc}
R_{q} \hbar|\omega|+R_{V}(e V-\hbar|\omega|), & \hbar|\omega|<e V, \\
R_{q} \hbar|\omega|, & \hbar|\omega|>e V,
\end{array}\right.
\end{aligned}
$$

where $V>0$ is voltage applied between left and right reservoirs. Here the electro-chemical capacitance is

$$
C_{\mu}^{-1}=C^{-1}+\left[e^{2}\left(N_{L}+N_{R}\right)\right]^{-1},
$$

the charge relaxation resistance ${ }^{10}$ reads

$$
R_{q}=\frac{\pi \hbar}{e^{2}} \sum_{\gamma \delta=L, R} \operatorname{Tr}\left(\mathcal{N}_{\gamma \delta} \mathcal{N}_{\gamma \delta}^{\dagger}\right)\left[\sum_{\gamma} \operatorname{Tr} \mathcal{N}_{\gamma \gamma}\right]^{-2},
$$

and the non-equilibrium resistance is

$$
R_{V}=\frac{2 \pi \hbar}{e^{2}} \operatorname{Tr}\left(\mathcal{N}_{L R} \mathcal{N}_{L R}^{\dagger}\right)\left[\sum_{\gamma} \operatorname{Tr} \mathcal{N}_{\gamma \gamma}\right]^{-2},
$$

with the notation

$$
\mathcal{N}_{\gamma \delta}=\frac{1}{2 \pi i} \sum_{\alpha} s_{\alpha \gamma}^{\dagger} \frac{\partial s_{\alpha \delta}}{\partial E} .
$$

It can be checked easily that equilibrium noise, $S_{G G}^{e q}=$ $2 C_{\mu}^{2} R_{q} \hbar|\omega|^{3}$, satisfies the fluctuation-dissipation theorem. The resistance $R_{q}$ can be extracted from the ac conductance as well. However, non-equilibrium noise is described by another resistance, $R_{V}$, which is a new quantity. It probes directly the non-diagonal density of states elements $\mathcal{N}_{L R}$ of the charge operator. The nondiagonal density of states elements which describe the charge fluctuations in a conductor in the presence of shot noise can be viewed as the density of states that is associated with a simultaneous current amplitude at contact $\gamma$ and contact $\delta$, regardless through which contact the carriers leave the sample. These density of states can be also viewed as blocks of the Wigner-Smith time delay matrix $(2 \pi i)^{-1} s^{\dagger} d s / d E$.

\footnotetext{
${ }^{40}$ In terms of the previous subsection, this is the charge relaxation resistance of the conductor. The charge relaxation resistance of the macroscopic gate is zero.
} 
For a saddle-point quantum point contact the resistances $R_{q}$ and $R_{V}$ are evaluated in Ref. [98. In the presence of a magnetic field $R_{V}$ has been calculated for a saddle point model by one of the authors and Martin [166], and is shown in Fig. 26. For a chaotic cavity connected to two single channel leads both resistances are random quantities, for which the whole distribution function is known [98]. Thus, the resistance $R_{q}$ (in units of $2 \pi \hbar / e^{2}$ ) assumes values between $1 / 4$ and $1 / 2$, with the average of $3 / 8$ (orthogonal symmetry) or $5 / 14$ (unitary symmetry). The resistance $R_{V}$ lies in the interval between 0 and $1 / 4$, and is on average $1 / 12$ and $1 / 14$ for orthogonal and unitary symmetry, respectively.

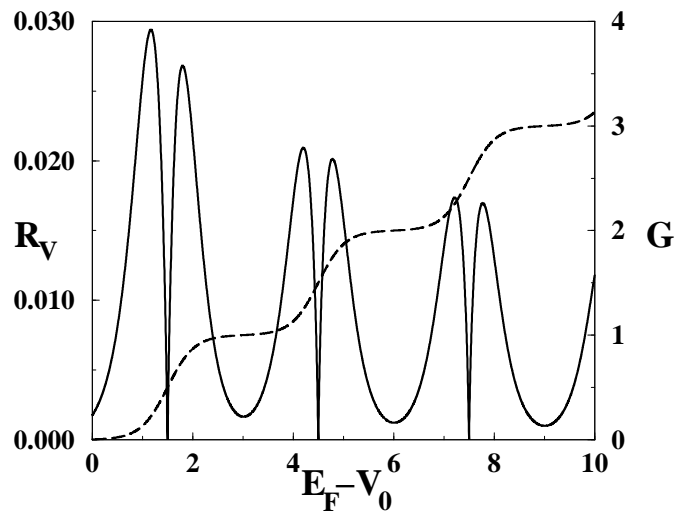

FIG. 26. $R_{V}$ (solid line, in units of $2 \pi \hbar / e^{2}$ ) and the conductance $G$ (dashed line, in units of $e^{2} / 2 \pi \hbar$ ) as a function of $E_{F}-V_{0}$ for a saddle point QPC with $\omega_{x} / \omega_{y}=1$ and $\omega_{c} / \omega_{x}=4, \omega_{c}$ is the cyclotron frequency. After Ref. [166.

\section{SHOT NOISE IN HYBRID NORMAL AND SUPERCONDUCTING STRUCTURES}

The dissipationless current (supercurrent) in superconductors is a property of a ground state, and therefore is noiseless - it is not accompanied by any fluctuations. However, noise appears if the superconductor is in contact with piece(s) of normal metals. This Section is devoted to the description of shot noise in these hybrid structures, which exhibit a variety of interesting phenomena. Following the point of view of the previous Sections, we present here a description which is based on an extension of the scattering approach to hybrid structures.

\section{A. Shot noise of normal-superconductor interfaces}

Simple NS interface, scattering theory and general expressions. We consider first an interface of normal metal and superconductor (NS). If the applied volt- age is below the superconducting gap $\Delta$, the only mechanism of charge transport is Andreev reflection at the NS interface: an electron with energy $E$ approaching the interface from the normal side is converted into a hole with energy $-E$. The velocity of the hole is directed back from the interface to a normal metal. The missing charge $2 e$ on the normal side appears as a new Cooper pair on the superconducting side. There is, of course, also a reverse process, when a Cooper pair recombines with a hole in the normal conductor, and creates an electron. At equilibrium, both processes have the same probability, and there is thus no net current. However, if a voltage is applied, a finite current flows across the NS interface.

The scattering theory which we described in Section II has to be extended to take into account the Andreev scattering processes. We give here only a sketch of the derivation; a more detailed description, as well as a comprehensive list of references, may be found in Refs. 72, 168]. To set up a scattering problem, we consider the following geometry (Fig. 27): The boundary between normal and superconducting parts is assumed to be sharp, and elastic scattering happens inside the normal metal (shaded region) only. The scattering region is separated from the NS interface by an ideal region 2, which is much longer than the wavelength, and thus we may there use asymptotic expressions for the wave functions. This spatial separation of scattering from the interface is artificial. It is not really necessary; our consideration leading to Eq. (159) and (160) does not rely on it. Furthermore, for simplicity, we assume that the number of transverse channels in the normal lead 1 and in the intermediate normal portion 2 is the same.

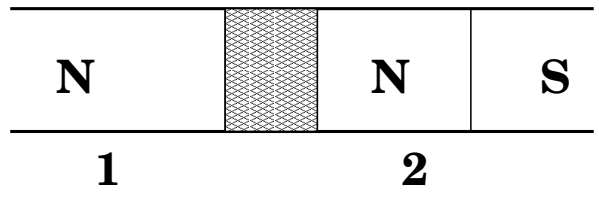

FIG. 27. A simplified model of an NS interface adopted for the scattering description. Scattering is assumed to happen in the shaded area inside the normal metal, which separates the ideal normal parts 1 and 2. Andreev reflection is happening strictly at the interface separating 2 and the superconductor.

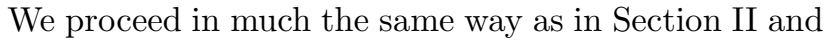
define the annihilation operators in the region 1 asymptotically far from the scattering area, $\hat{a}_{1 e n}$, which annihilate electrons incoming on the sample. These electrons are described by wave functions $\chi_{1 n}\left(\boldsymbol{r}_{\perp}\right) \exp \left(i k_{F} z\right)$ with unit incident amplitude, where the coordinate $z$ is directed towards the superconductor (from the left to the right in Fig. 27), the index $n$ labels the transverse channels. Here we neglected the energy dependence of the wave vector, anticipating the fact that only energies close to the Fermi surface will play a role in transport. Similarly, the operator $\hat{b}_{1 e n}$ annihilates electrons in the outgoing states in the region $1, \chi_{1 n}\left(\boldsymbol{r}_{\perp}\right) \exp \left(-i k_{F} z\right)$. 
For holes, we define an annihilation operator in the incoming states in the region 1 as $\hat{a}_{1 h n}$, and the corresponding wave function is $\chi_{1 n}\left(\boldsymbol{r}_{\perp}\right) \exp \left(-i k_{F} z\right)$. Note that though this wave function is identical to that for outgoing electrons, it corresponds to the incoming state with energy $-E$. The velocity of these holes is directed towards the interface. The annihilation operator for holes in the outgoing states, $\hat{b}_{1 h n}$, is associated with the wave function $\chi_{1 n}\left(\boldsymbol{r}_{\perp}\right) \exp \left(i k_{F} z\right)$. Creation operators for electrons and holes are defined in the same way. Thus, the difference with the scattering theory for normal conductors is that we now have an extra index, which assumes values $e, h$ and discriminates between electrons and holes.

The electron and hole operators for the outgoing states are related to the electron and hole operators of the incoming states via the scattering matrix,

$$
\left(\begin{array}{l}
\hat{b}_{1 e} \\
\hat{b}_{1 h}
\end{array}\right)=s\left(\begin{array}{l}
\hat{a}_{1 e} \\
\hat{a}_{1 h}
\end{array}\right) \equiv\left(\begin{array}{ll}
s_{e e} & s_{e h} \\
s_{h e} & s_{h h}
\end{array}\right)\left(\begin{array}{l}
\hat{a}_{1 e} \\
\hat{a}_{1 h}
\end{array}\right),
$$

where the element $s_{e e}$ gives the outgoing electron current amplitude in response to an incoming electron current amplitude, $s_{\text {he }}$ gives the outgoing hole current amplitude in response to an incoming electron current amplitude, etc. The generalized current operator (32) for electrons and holes in region 1 is

$$
\begin{aligned}
\hat{I}_{L}(t) & =\frac{e}{2 \pi \hbar} \int_{0}^{\infty} d E d E^{\prime} e^{i\left(E-E^{\prime}\right) t / \hbar} \\
& \times \operatorname{Tr}\left[\hat{a}_{1 e}^{\dagger}(E) \hat{a}_{1 e}\left(E^{\prime}\right)-\hat{a}_{1 h}^{\dagger}(E) \hat{a}_{1 h}\left(E^{\prime}\right)\right. \\
& \left.-\hat{b}_{1 e}^{\dagger}(E) \hat{b}_{1 e}\left(E^{\prime}\right)+\hat{b}_{1 h}^{\dagger}(E) \hat{b}_{1 h}\left(E^{\prime}\right)\right],
\end{aligned}
$$

or, equivalently,

$$
\begin{aligned}
\hat{I}_{L}(t) & =\frac{e}{2 \pi \hbar} \sum_{\alpha \beta} \int_{0}^{\infty} d E d E^{\prime} e^{i\left(E-E^{\prime}\right) t / \hbar} \\
& \times \operatorname{Tr}\left[\hat{a}_{1 \alpha}^{\dagger}(E) A_{\alpha \beta}\left(E, E^{\prime}\right) \hat{a}_{1 \beta}\left(E^{\prime}\right)\right],
\end{aligned}
$$

where we have again introduced electron-hole indices $\alpha$ and $\beta$, and the trace is taken over channel indices. The matrix $A$ is given by

$$
A\left(E, E^{\prime}\right)=\Lambda-s^{\dagger}(E) \Lambda s\left(-E^{\prime}\right), \quad \Lambda=\left(\begin{array}{cc}
1 & 0 \\
0 & -1
\end{array}\right),
$$

with the matrix $\Lambda$ discriminating between electron and holes. Introducing the distribution functions for electrons $f_{e}(E)=\left[\exp \left[(E-e V) / k_{B} T\right]+1\right]^{-1}$ and holes $f_{h}(E)=\left[\exp \left[(E+e V) / k_{B} T\right]+1\right]^{-1}$, and acting in a similar way as in Section II for normal systems, we obtain from the current operator and the usual quantum statistical assumptions for the averages and correlations of the electron and hole operators in the normal reservoir the zero-temperature conductance

$$
G=\frac{e^{2}}{\pi \hbar} \operatorname{Tr}\left[s_{h e}^{\dagger} s_{h e}\right]
$$

and the shot noise power

$$
S=\frac{4 e^{3}|V|}{\pi \hbar} \operatorname{Tr}\left[s_{h e}^{\dagger} s_{h e}\left(1-s_{h e}^{\dagger} s_{h e}\right)\right]
$$

in the zero-temperature limit up to linear order in the applied voltage. Here we have made use of the unitarity of the scattering matrix, in particular, $s_{e e}^{\dagger} s_{e e}+s_{h e}^{\dagger} s_{h e}=1$, and of the particle-hole symmetry. As a consequence, both the conductance and the noise can be expressed in terms of $s_{h e}$ only. We emphasize that Eqs. (159) and (160) are completely general: In particular, they do not require a clean NS interface and the spatial separation of the scattering region of the normal conductor from the interface. However, without such additional assumptions the evaluation of the scattering matrix can be very difficult.

To gain more insight we now follow Beenakker [72 and assume, as shown in Fig. 27, that a perfect region of normal conductor is inserted between the disordered part of the conductor and the NS-interface. In region 2, incoming states for electrons and outgoing states for holes have wave functions proportional to $\exp \left(-i k_{F} z\right)$, while outgoing states for electrons and incoming states for holes contain the factor $\exp \left(i k_{F} z\right)$. We also define annihilation operators $\hat{a}_{2 e n}, \hat{a}_{2 h n}, \hat{b}_{2 e n}, \hat{b}_{2 h n}$, and creation operators for this region.

The scattering inside the normal lead is described by a $4 N_{\perp} \times 4 N_{\perp}$ scattering matrix $s_{N}\left(N_{\perp}\right.$ being the number of transverse channels),

$$
\left(\begin{array}{l}
\hat{b}_{1 e} \\
\hat{b}_{2 e} \\
\hat{b}_{1 h} \\
\hat{b}_{2 h}
\end{array}\right)=s_{N}\left(\begin{array}{l}
\hat{a}_{1 e} \\
\hat{a}_{2 e} \\
\hat{a}_{1 h} \\
\hat{a}_{2 h}
\end{array}\right),
$$

where operators like $\hat{b}_{1 e}$ are vectors, each component denoting an individual transverse channel. The elastic scattering in the normal region does not mix electrons and holes, and therefore in the electron-hole decomposition the matrix $s_{N}$ is diagonal,

$$
s_{N}=\left(\begin{array}{cc}
s_{0}(E) & 0 \\
0 & s_{0}^{*}(-E)
\end{array}\right), \quad s_{0}(E)=\left(\begin{array}{cc}
r_{11} & t_{12} \\
t_{21} & r_{22}
\end{array}\right),
$$

Here $s_{0}(E)$ is the usual $2 N_{\perp} \times 2 N_{\perp}$ scattering matrix for electrons, which contains reflection and transmission blocks.

To leading order in $\Delta / E_{F}$ (if both the normal conductors and the superconductor have identical Fermi energies) Andreev reflection at a clean interface is described by a $2 N \times 2 N$ scattering matrix, which is off-diagonal in the electron-hole decomposition, and is given by

$$
\left(\begin{array}{l}
\hat{a}_{2 e} \\
\hat{a}_{2 h}
\end{array}\right)=\gamma\left(\begin{array}{cc}
0 & \exp (i \phi) \\
\exp (-i \phi) & 0
\end{array}\right)\left(\begin{array}{l}
\hat{b}_{2 e} \\
\hat{b}_{2 h}
\end{array}\right)
$$

with $\gamma \equiv \exp [-i \arccos (E / \Delta)]$. With some algebra we can now find expressions for the scattering matrices 
$s_{e e}, s_{e h}, \ldots$ in terms of the Andreev reflection amplitude and the scattering matrix of the normal region [72],

$$
\begin{aligned}
s_{e e}(E) & =r_{11}(E)+\gamma^{2} t_{12}(E) r_{22}^{*}(-E) \\
& \times\left[1-\gamma^{2} r_{22}(E) r_{22}^{*}(-E)\right]^{-1} t_{21}(E), \\
s_{e h}(E) & =\gamma \exp (i \phi) t_{12}(E) \\
& \times\left[1-\gamma^{2} r_{22}^{*}(-E) r_{22}(E)\right]^{-1} t_{21}^{*}(-E), \\
s_{h e}(E) & =\gamma \exp (-i \phi) t_{12}^{*}(-E) \\
& \times\left[1-\gamma^{2} r_{22}(E) r_{22}^{*}(-E)\right]^{-1} t_{21}(E), \\
s_{h h}(E) & =r_{11}^{*}(-E)+\gamma^{2} t_{12}^{*}(-E) r_{22}(E) \\
& \times\left[1-\gamma^{2} r_{22}^{*}(-E) r_{22}(E)\right]^{-1} t_{21}^{*}(-E) .
\end{aligned}
$$

These matrices express amplitudes for an electron (hole) incoming from the left to be eventually reflected as an electron (hole). The corresponding probability is given by the squared absolute value of the matrix element. In the following, we only consider the case when no magnetic field is applied to the structure. Then the matrix $s_{0}(E)$ is symmetric. For $e|V| \ll \Delta$ one has $\gamma=-i$. Taking again the particle-hole symmetry into account, we obtain with the help of Eqs. (159), (162) the conductance

$$
G=\frac{e^{2}}{\pi \hbar} \sum_{n} \frac{T_{n}^{2}}{\left(2-T_{n}\right)^{2}},
$$

and using Eq. (160), the shot noise

$$
\begin{aligned}
S & =\frac{4 e^{3}|V|}{\pi \hbar} \operatorname{Tr}\left[s_{h e}^{\dagger} s_{h e}\left(1-s_{h e}^{\dagger} s_{h e}\right)\right] \\
& =\frac{16 e^{3}|V|}{\pi \hbar} \sum_{n} \frac{T_{n}^{2}\left(1-T_{n}\right)}{\left(2-T_{n}\right)^{4}},
\end{aligned}
$$

where $T_{n}$ are eigenvalues of the matrix $t_{12}^{\dagger} t_{12}$, i.e. transmission eigenvalues of the normal region (evaluated at the Fermi surface). As in normal conductors, channels with $T_{n}=0$ and $T_{n}=1$ do not contribute to the noise. Note that it is the fact that we have chosen to express the conductance and the noise in terms of the eigenvalues of the normal region which gives rise to the non-linear eigenvalue expressions given by Eqs. (164), (165). In terms of the eigen channels of $s_{h e}$ the resulting expression would be formally identical to the conductance and the noise of a normal conductor.

The expression (165) was obtained by Khlus [15] using a Keldysh approach for the case when the normal metal and the superconductor are separated by a tunnel barrier. He also investigated the finite temperature case and derived the Nyquist noise. The results were rederived within the scattering approach by Muzykantskii and Khmelnitskii 169. The general case was studied by de Jong and Beenakker [170] in the framework of the scattering approach; we followed their work in the course of the above derivation. Martin [171] obtains the same results using statistical particle counting arguments and investigates the crossover between shot and thermal noise (see below).

Applications. If the normal and superconducting electrodes are separated by a tunnel barrier, all the transmission coefficients $T_{n}$ can be taken to be the same, $T_{n}=T$ (not to be confused with temperature). Then we obtain

$$
\begin{aligned}
G & =\frac{e^{2} N_{\perp}}{\pi \hbar} \frac{T^{2}}{(2-T)^{2}} \\
S & =\frac{16 e^{3}|V| N_{\perp}}{\pi \hbar} \frac{T^{2}(1-T)}{(2-T)^{4}}
\end{aligned}
$$

with $N_{\perp}$ the number of transverse channels. For the Fano factor this yields 15,170

$$
F=\frac{S}{2 e G V}=\frac{8(1-T)}{(2-T)^{2}} .
$$

For low transparency $T \ll 1$ the Fano factor tends to the value of 2 . This corresponds to the notion that shot noise in NS junctions is essentially the result of uncorrelated transfer of particles with charge $2 e$. The shot noise is super-Poissonian $(F>1)$ for $T<2(\sqrt{2}-1) \approx 0.83$. For open barriers $(T=1)$ the shot noise vanishes.

Refs. 15, 171 have also shown that the crossover between shot and Nyquist noise happens at the temperature $k_{B} T=2 e|V|$, which is one more manifestation of the doubling of the effective charge.

For the case of a disordered normal metal, Eqs. (164) and (165) have to be averaged over impurity configurations. Using the distribution function of the transmission coefficients in the disordered region (87), we obtain for the Fano factor 170 $F=2 / 3$. This is twice as high as for a normal disordered wire. Macêdo 85] obtains the weak localization correction and mesoscopic fluctuations of the shot noise power. In particular, he finds that the mean square of the shot noise power scales as the shot noise power itself, and in this sense the fluctuations are universal (as for normal diffusive conductors).

De Jong and Beenakker [170 analyze the case when both a disordered normal metal and tunnel barrier are present, and describe the crossover between the two limiting regimes which are obtained in the absence of a disordered region or in the absence of tunnel barrier.

Naidenov and Khlus 172 and Fauchère, Lesovik, and Blatter 173 analyze the situation when the normal and the superconducting electrodes are separated by a resonant double barrier (in particular, this may correspond to the situation of resonant impurities in the insulating layer separating the two electrodes). Ref. 172 considers the one-channel sample specific case (no averaging) and discussed the resonant structure of the conductance and the noise. Using the distribution function of transmission eigenvalues and assuming that the barrier is symmetric, Ref. 173 finds for the ensemble average a Fano factor $F=3 / 4$. This should be contrasted with the result $F=1 / 2$ for the corresponding normal symmetric resonant double barrier. 
Schep and Bauer 93 investigate the effect of a disordered interface separating the normal metal and the superconductor. The Fano factor also, of course, equals $F=3 / 4$, which is higher than the value $2 / 3$ discussed above.

Non-linear regime. Khlus [15], and subsequently Anantram and Datta [174] (who used the scattering approach), considered noise in the non-linear regime. Without giving details, we only mention the case of an ideal NS interface coupled to a perfect wire for which all the transmission coefficients $T_{n}$ are equal to one. This ideal contact does not exhibit shot noise for voltages below $\Delta / e$, as is seen from Eq. (165). The physical reason is that in this case the scattering process is not random: an electron approaching the interface is converted into a hole with probability one and sent back. However, as the voltage increases above $\Delta / e$, quasiparticle states in the superconductor become available, and electrons can now tunnel into the superconductor without being reflected as holes (imperfect Andreev reflections). This induces noise even for an ideal interface. For still higher voltages, an even broader range of energies is involved. However, for energies $E \gg \Delta$ (almost) all electrons tunnel into the superconductor without being Andreev reflected. Since the interface is ideal, this process is also noiseless. Thus, noise is produced only by electrons with energies higher than $\Delta$, but with energies which are in the vicinity of $\Delta$. This implies 15, 174 that the shot noise is zero for $e|V|<\Delta$, then starts to grow rapidly, and saturates when the voltage becomes of the order of several $\Delta / e$. The saturation value is found [15] to be

$$
S_{\max }=\frac{4 e^{2} N_{\perp} \Delta}{15 \pi \hbar}
$$

For a non-ideal conductor $\left(0<T_{n}<1\right.$ for at least one channel) the same mechanism leads to the crossover from Eq. (165) at low voltages to Eq. (57) for high voltages.

For barriers of low transparency $T \ll 1$ (for instance, when there is an insulating layer at some distance from the NS interface) another mechanism for non-linear noise takes place, as discussed by Fauchère, Lesovik and Blatter [173]. In such a geometry the phases of the scattering matrix are energy sensitive. For $e|V| \ll \Delta$ we obtain, similarly to Eqs. (164) and (165), formulae for non-linear transport,

$$
I=\frac{e}{\pi \hbar} \sum_{n} \int_{0}^{e V} \frac{T_{n}^{2}}{T_{n}^{2}+2\left(1-T_{n}\right)\left(1-\cos \left(\alpha_{n}(E)\right)\right.} d E,
$$

and noise,

$$
S=\frac{8 e^{2}}{\pi \hbar} \sum_{n} \int_{0}^{e|V|} \frac{T_{n}^{2}\left(1-T_{n}\right)\left(1-\cos \left(\alpha_{n}(E)\right)\right.}{\left[T_{n}^{2}+2\left(1-T_{n}\right)\left(1-\cos \left(\alpha_{n}(E)\right)\right]^{2}\right.} d E
$$

Here we assumed the transmission probabilities $T_{n}$ to be energy independent. The phase $\alpha_{n}$ is

$$
\begin{aligned}
\alpha_{n}(E) & =\phi_{n}(E)-\phi_{n}(-E)-2 \arccos (E / \Delta) \\
& =4 E d / \hbar v_{n}-2 \arccos (E / \Delta)
\end{aligned}
$$

where $d$ is the distance between the insulating layer and the NS interface, and $v_{n}$ is the velocity in the channel $n$. $\phi_{n}(E)$ is the phase that an electron with energy $E$ acquires during a round-trip between the NS interface and the insulating layer. For $E=0$ we have $\alpha_{n}=\pi$, and thus in the linear regime Eqs. 168) and (169) are reduced to Eqs. (164) and (165), respectively.

These expressions can be interpreted as follows. The part of the normal metal between the NS interface and the tunnel barrier serves as an Andreev resonant double barrier. The electron entering this region travels to the NS interface, is converted into a hole, then this hole makes a round-trip, and is converted to an electron, which returns to the barrier. The total phase gain during this trip is $\alpha_{n}(E)$. The "transmission probability" of this process (the integrand in Eq. (168)) shows a pronounced resonance structure near the energies where the phase $\alpha_{n}(E)$ equals $2 \pi m$ with integer $m$. Explicitly, for each channel $n$, we have a set of resonances (Andreev - Kulik bound states 173) $E_{m}=\left(\pi \hbar v_{n} / 4 d\right)(2 m+1)$. Thus, the behavior of the transmission probability is similar to that describing resonant tunneling in the double barrier normal system.

Specializing further to the case of one channel with velocity $v_{F}$ and transmission coefficient $T \ll 1$, we write the analog of the Breit-Wigner formula

$$
I=\frac{e T^{2}}{\pi \hbar} \sum_{m} \int_{0}^{e V} \frac{d E}{T^{2}+\left(4 d / \hbar v_{F}\right)^{2}\left(E-E_{m}\right)^{2}}
$$

and

$$
\begin{aligned}
S & =\frac{4 e^{2} T^{2}}{\pi \hbar}\left(\frac{4 d}{\hbar v_{F}}\right)^{2} \sum_{m} \\
& \times \int_{0}^{e|V|} \frac{\left(E-E_{m}\right)^{2} d E}{\left[T^{2}+\left(4 d / \hbar v_{F}\right)^{2}\left(E-E_{m}\right)^{2}\right]^{2}} .
\end{aligned}
$$

We see that both the current and the noise power show plateaus as a function of applied voltage; sharp transitions between the plateaus take place at resonances, when $e|V|=E_{m}$. In particular, when the voltage $e V$ lies between the resonances (plateau regime), $E_{M}<e|V|<$ $E_{M+1}$, we have $I=e T v_{F} /(4 d)$, and $S=2 e I$. Thus, already after the first resonance, the Fano factor assumes the value $F=1$, the same as for the normal structure. The explanation is that the transport through Andreev bound states, which dominates in this regime, is not accompanied with the formation of Cooper pairs, and thus the usual classical Schottky value is restored. These considerations should be supplemented by an analysis of the charge and its fluctuations and the role of screening (see Section $\mathrm{V}$ ). 
ND interfaces. Zhu and Ting [175] considered shot noise of the interface between a normal metal and a superconductor with a $d$-wave symmetry. For this purpose, they generalized the scattering approach for this situation and subsequently performed numerical studies. Now the shot noise depends on the orientation of the superconducting order parameter at the interface. Zhu and Ting [175] investigated only one particular orientation, when the gaps felt by electrons and holes are of the same magnitude but of different signs.

The results they found are drastically different from those for $s$-wave superconductors. In the tunneling regime, the Fano factor is zero (rather than 2) for low voltages. It grows with voltage and saturates at $F=1$ for $e|V| \gg \Delta$. Thus, shot noise is below the Poisson value, defined with respect to the normal metal, for all voltages. In contrast, in the ballistic limit the Fano factor is enhanced as compared with $s$-wave superconductors.

To our knowledge, Ref. [175] is the only paper addressing shot noise in hybrid structures with non-trivial symmetry of the order parameter.

Frequency dependence. The frequency dependence of the noise of NS interfaces is easy to find in the situation when the scattering matrices of the normal region may be assumed to be energy independent. Lesovik, Martin and Torrès 176] have investigated this case. Generalizing Eq. (125) to the case of NS interface, we write

$$
\begin{aligned}
S(\omega) & =\frac{e^{2}}{\pi \hbar}\left\{\sum_{n} D_{n}^{2} \int d E\left[f_{e e}(E, \omega)+f_{h h}(E, \omega)\right]\right. \\
& \left.+\sum_{n} D_{n}\left(1-D_{n}\right) \int d E\left[f_{e h}(E, \omega)+f_{h e}(E, \omega)\right]\right\},
\end{aligned}
$$

with $D_{n} \equiv T_{n}^{2}\left(2-T_{n}\right)^{-2}$. Performing the integration, we obtain

$$
\begin{aligned}
& S(\omega)=\frac{e^{2}}{\pi \hbar}\left\{2 \hbar \omega \operatorname{coth}\left(\frac{\hbar \omega}{2 k_{B} T}\right) \sum_{n} D_{n}^{2}\right. \\
+ & {\left[(\hbar \omega+2 e V) \operatorname{coth}\left(\frac{\hbar \omega+2 e V}{2 k_{B} T}\right)\right.} \\
+ & \left.\left.(\hbar \omega-2 e V) \operatorname{coth}\left(\frac{\hbar \omega-2 e V}{2 k_{B} T}\right)\right] \sum_{n} D_{n}\left(1-D_{n}\right)\right\},
\end{aligned}
$$

which gives the noise frequency spectrum for arbitrary frequencies, voltages, and temperatures (provided all of them are much below $\Delta$ ). For $V=0$ Eq. (173) agrees with the fluctuation-dissipation theorem. At zero temperature, we obtain 176]

$$
\begin{gathered}
S(\omega)=\frac{2 e^{2}}{\pi \hbar} \\
\times\left\{\begin{array}{cc}
\hbar|\omega| \sum_{n} D_{n}^{2}+2 e|V| \sum_{n} D_{n}\left(1-D_{n}\right), & \hbar|\omega|<2 e|V|, \\
\hbar|\omega| \sum_{n} D_{n}, & \hbar|\omega|>2 e|V| .
\end{array}\right.
\end{gathered}
$$

This expression is quite similar to Eq. (129) which describes zero temperature noise frequency spectrum in the normal contact. One evident difference is that the transmission coefficients $T_{n}$ are replaced by $D_{n}$, due to the modification of scattering by Andreev reflections. Another observation is that the electron charge is now doubled. Thus, instead of the singularity at the frequency $\hbar \omega= \pm \mathrm{eV}$ in a normal metal we have now the singularity at $\hbar \omega= \pm 2 \mathrm{eV}$. This is yet one more manifestation of the fact that transport in NS structures is related to the transmission of Cooper pairs.

Even more interesting effects are expected when the frequency becomes of order $\Delta$. In this case the total scattering matrix, however, can by no means assumed to be energy independent, and the self-consistent treatment of interactions ${ }^{17}$ is needed, as we discussed in Section III. A step in this direction has been done in Ref. [177], which analyzes noise of a NS interface measured at a capacitively coupled gate and only considers the charge self-consistency. In accordance with the general conclusions of Section III, the leading order in frequency for this noise is given by $S_{G G}=2 C_{\mu}^{2} \omega^{2} R_{V} e|V|$, where $R_{V}$ is determined by the properties of the interface. In particular, when the normal part contains a quantum point contact and a new channel opens, $R_{V}=9 \pi \hbar / 2 e^{2}$, whereas for a normal quantum point contact $R_{V}=0$ in this situation.

Multi-terminal devices. Consider now a multiprobe hybrid structure, which contains a number of normal and a number of superconducting leads (the superconducting leads are taken at the same chemical potential). The current operator Eq. (157) can be written for each lead of a multi-terminal structure connected to a superconductor. This leads to a second quantization formulation of the current-current correlations put forth by Anantram and Datta 174]. At each normal contact, labeled $\alpha$, the current is the sum of an electron current $I_{\alpha}^{e}$ and a hole current $I_{\alpha}^{h}$. In terms of the scattering matrix the resulting current correlations are

$$
\begin{aligned}
\left\langle\Delta I_{\alpha}^{\mu} \Delta I_{\beta}^{\nu}\right\rangle & =\frac{q^{\mu} q^{\nu}}{\pi \hbar} \sum_{\substack{\gamma \lambda \\
\delta \kappa}} \int_{0}^{\infty} d E \operatorname{Tr}\left[A_{\gamma \lambda, \delta \kappa}(\alpha \mu) A_{\delta \kappa, \gamma \lambda}(\beta \nu)\right] \\
& \times f_{\gamma}^{\lambda}(E)\left[1-f_{\delta}^{\kappa}(E)\right],
\end{aligned}
$$

where $q^{e}=-e$ and $q^{h}=e$. Here the indices $\alpha, \beta, \gamma, \delta$ label the terminals, and $\kappa, \lambda, \mu, \nu$ describe the electron-hole decomposition and may assume values $e$ and $h$. Basing on Eq. (175), Anantram and Datta predict that, though correlations at the same contact are always positive, like in the case of normal structures, those at different contacts also may in certain situations become positive. (We remind the reader that cross-correlations are quite generally negative in normal devices, as discussed in Sec-

\footnotetext{
${ }^{41}$ By this now we mean self-consistency in both the charge and the superconducting order parameter $\Delta$.
} 
tion [II). Similar results have been obtained by Martin [171 using statistical particle counting arguments.

Qualitatively, this conclusion may be understood in the following way. There are two types of processes contributing to noise. First, an electron or hole can be simply reflected from the interface without being Andreev reflected. In accordance with the general considerations, this reflection tends to make the cross-correlation negative. On the other hand, processes involving Andreev reflection (an electron is converted into a hole or vice versa) provide transport of particles with opposite charges. Due to Eq. (175), these processes are expected to push the cross-correlations towards positive values. This interplay between normal scattering and Andreev reflections determines the total sign of the cross-correlations. This is, indeed, seen from the expressions of Anantram and Datta 174, who decompose current correlations at different contacts into a sum of positive definite and negative definite contributions.

The interpretation which may be found in the literature, that positive cross-correlations in hybrid structures are due to the bosonic nature of Cooper pairs, does not seem to be plausible. Indeed, the microscopic theory of superconductivity never uses explicitly the Bose statistics of Cooper pairs. In particular, Eq. (175) only contains the (Fermi) distribution functions of electrons and holes, but not the distribution function of Cooper pairs.

Quantitative analysis of this effect would also require the next step, which is to express the scattering matrix $s$ through the scattering matrices of the normal part of the device (multi-terminal analog of Eq. (163)). The multiterminal correlations could then be studied for various systems, similarly to the discussion for the normal case (see Section II). Analytical results are currently only available for systems with an ideal NS interface, where the matrix $s$ is fully determined by Andreev reflection.

Anantram and Datta [174] consider a three-terminal device with two normal contacts and a contact to the superconductor. The superconductor connects to the normal system via two NS-interfaces forming a loop which permits the application of an Aharonov-Bohm flux. The conductor is a perfect ballistic structure and the NS interfaces are also taken to be ideal. In this system, shot noise is present for arbitrary voltages, since the electron emitted from the normal contact 1, after (several) Andreev reflections may exit through the normal contact 1 or 2, as an electron or as a hole. Specifically, Ref. 174 studies cross-correlations of current at the two normal contacts, and finds that they may be both positive and negative, depending on the phases of Andreev reflection which in their geometry can be tuned with the help of an Aharonov-Bohm flux.

Another three-terminal geometry, a wave splitter connected to a superconductor, is discussed by Martin 171 and Torrès and Martin [178. The cross-correlation in the normal leads depends on the parameter $\epsilon$ which describes the coupling to the superconducting lead (see Eq. (113)), $0<\epsilon<1 / 2$ 120,121]. For an ideal NS interface,
Ref. 178 finds that the cross-correlations are positive for $0<\epsilon<\sqrt{2}-1$ (weak coupling) and negative for $\sqrt{2}-1<\epsilon<1 / 2$. Torrés and Martin 178 also report numerical results for disordered NS interfaces, showing that disorder enhances positive cross-correlations.

A particularly instructive example has been analyzed in Ref. 179 by Gramespacher and one of the authors of this Review. They investigate the shot noise measurement with a tunneling contact (STM tip) which couples very weakly to a normal conductor which is in turn coupled to a superconductor. If both the normal reservoir and the superconductor are taken at the same potential $\mu_{0}$, and the tunneling tip at potential $\mu$, they find from Eq. (175) the following correlations,

$$
\begin{aligned}
& \left\langle\Delta I_{1}^{e} \Delta I_{\text {tip }}^{e}\right\rangle=\alpha 4 \pi^{2} \nu_{t i p}|t|^{2} \nu\left(x_{e}, 1_{e}\right), \\
& \left\langle\Delta I_{1}^{h} \Delta I_{\text {tip }}^{e}\right\rangle=-\alpha 4 \pi^{2} \nu_{t i p}|t|^{2} \nu\left(x_{e}, 1_{h}\right), \\
& \left\langle\Delta I_{1}^{e} \Delta I_{\text {tip }}^{h}\right\rangle=\left\langle\Delta I_{1}^{h} \Delta I_{\text {tip }}^{h}\right\rangle=0,
\end{aligned}
$$

with $\alpha=-\left(e^{2} / \pi \hbar\right) \Delta \mu, \Delta \mu=\mu-\mu_{0}$, and $|t|^{2}$ the coupling energy of the tip to the sample. Here $\nu\left(x_{e}, 1_{e}\right)$ is the electron density generated at the coupling point $x$ due to injected electrons and $\nu\left(x_{e}, 1_{h}\right)$ is the electron density at the coupling point due to holes injected by the normal reservoir. The total correlation of the currents at contact 1 and 2 is the sum of all four terms. In the absence of a magnetic field, the correlations are proportional to the injected net charge density $q(x)=\nu\left(x_{e}, 1_{e}\right)-\nu\left(x_{h}, 1_{e}\right)$, and given by

$$
\begin{aligned}
\left\langle\Delta I_{1} \Delta I_{t i p}\right\rangle & =-\frac{e^{2}}{\pi \hbar} \Delta \mu 4 \pi^{2} \nu_{t i p}|t|^{2} q(x) \\
& =-2 G_{0} \Delta \mu \frac{q(x)}{p(x)}
\end{aligned}
$$

where $p(x)=\nu\left(x_{e}, 1_{e}\right)+\nu\left(x_{h}, 1_{e}\right)$ is the total particle density of states and $G_{0}=\left(e^{2} / 2 \pi \hbar\right) 4 \pi^{2} \nu_{t i p}|t|^{2} p(x)$ is the tip to sample conductance. This result states, that if at the point $x$ the electrons injected from contact 1 generate a hole density at $x$ which is larger than the electron density at $x$, the injected charge becomes negative and the corresponding correlation becomes positive. A more detailed analysis suggest that this effect is of order $1 / N$, where $N$ is the number of channels.

Up to now positive correlations in hybrid structures have been theoretically demonstrated only for single channel conductors. This leaves open the question, on whether or not, ensemble averaged shot noise spectra can in fact have a positive sign in hybrid structures.

Experiments. The only experiment on shot noise in NS structures currently available was performed by Vystavkin and Tarasov 180 long before the current interest on shot noise in mesoscopic systems started. For this reason, they did not study noise systematically, and only concluded that in certain samples it was suppressed below the value $2 e\langle I\rangle$.

Recently, Jehl et al 181 experimented with an $\mathrm{Nb} / \mathrm{Al} / \mathrm{Nb}$ structure at temperatures above the critical 
temperature for $\mathrm{Al}$, but below that for $\mathrm{Nb}$. They estimate that the length of the $\mathrm{Al}$ region was longer than the thermal length, which means that the multiple Andreev reflection processes (see below) are suppressed. Thus, qualitatively their SNS structure acts just as two incoherent NS interfaces, and the expected effective charge is $2 e$ (the Fano factor for the diffusive system is $2 / 3$ ). Indeed, the measurements show that the Fano factor for high temperatures is $1 / 3$ for all voltages (in accordance with the result for a metallic diffusive wire), while for lower temperatures it grows. The low-temperature behavior is found to be in better agreement with the value $F=2 / 3$ for an NS interface, than with the $F=1 / 3$ prediction for normal systems, though the agreement is far from perfect. The experimental results are shown in Fig. 28.

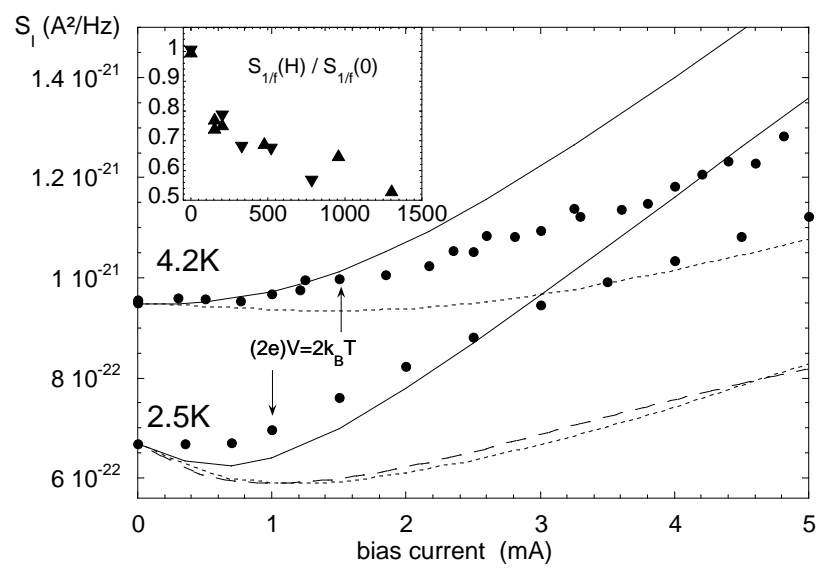

FIG. 28. Experimental results of Jehl et al 181. Solid and dotted lines are theoretical curves corresponding to the effective charges $2 e$ and $e$, respectively.

A clear experimental demonstration of the shot noise doubling with clean NS interfaces remains to be performed.

\section{B. Noise of Josephson junctions}

Josephson junctions are contacts which separate two superconducting bulk electrodes by an insulating barrier. We briefly describe here noise properties for the case when the transmission of this barrier is quite low; other, more interesting, cases are addressed in the next subsection.

The transport properties of Josephson junctions can be summarized as follows. First, at zero voltage a Josephson current may flow across the junction, $I=I_{0} \sin \phi$, where $\phi$ is the difference of the phases of the superconducting order parameter between the two electrodes. In addition, for finite voltage tunneling of quasiparticles between the electrodes is possible. For zero temperature this quasiparticle current only exists when the voltage exceeds $2 \Delta / e$; for finite temperature an (exponentially small) quasiparticle current flows at any voltage.

The Josephson current is a property of the ground state of the junction, and therefore it does not fluctuate. Hence, shot noise in Josephson junctions is due to the quasiparticle current ${ }^{12}$, and basically coincides with the corresponding shot noise properties of normal tunnel barriers. For zero temperature, there is no shot noise for voltages below $2 \Delta / e$. Thermal and shot noise in Josephson junctions are analyzed in detail by Rogovin and Scalapino [26], and have been measured by Kanter and Vernon, Jr. 183,184].

For completeness, we mention that if a voltage $V(t)$ is applied across the junction, as a consequence of gauge invariance, the Josephson current becomes timedependent,

$$
I(\phi)=I_{0} \sin \left[\phi+\frac{2 e}{\hbar} \int_{0}^{t} V(t) d t\right] .
$$

Then, due to any fluctuations of the voltage $V(t)$ (like thermal and shot noise) the Josephson junction starts to radiate. If the voltage $V$ is time-independent on average, the spectral density of this radiation is centered around the resonant frequencies $\omega=2 \mathrm{enV} / \hbar$, and fluctuations determine the width of the maxima, the linewidth of the Josephson radiation. This effect, analyzed by Stephen [185,186, turned out to be an effective experimental tool for detecting voltage fluctuations in Josephson junctions. It is the subject of many theoretical [23,24, 187,26, 188 191 and experimental [192,24, 193 195] papers.

\section{Noise of SNS hybrid structures}

Now we address the limit in which the two superconducting electrodes are separated by a region in which the motion is ballistic, or, at least, the transmission probability is not too small; for definiteness we will consider the quantum point contact connected to superconducting banks. To make a distinction between the tunnel Josephson junction described in the previous subsection and the case of interest here, we refer to these systems as SNS structures; for convenience, we only describe the one-channel case, and first consider the perfect contact for which the transmission probability is equal to one. Furthermore, we consider the case of a constriction with a length (distance between superconducting electrodes) small compared to the superconducting coherence length.

\footnotetext{
${ }^{42}$ As stated by Likharev in his 1979 review 182, "the most important results of all the theories of fluctuations in the Josephson effect is that the only intrinsic source of fluctuations is the normal current rather than the supercurrent of the junction".
} 
Equilibrium noise. In many respects, SNS structures are different from the tunnel Josephson junctions. A phase-coherent SNS structure supports a discrete set of subgap states. Carriers are trapped between two NS interfaces which act as Andreev mirrors. An electron reaching one of the interfaces is reflected as a hole and travels back to the other interface where it is reflected as an electron. The subgap states are known as AndreevKulik states [196]. In the particular case of interest here, there are two subgap states [197] with energies $\epsilon_{ \pm}(\phi)= \pm \Delta \cos (\phi / 2)$, which carry Josephson current. These states have a width $\gamma(\phi) \equiv \gamma[\epsilon(\phi)]$, which can appear, for instance, due to electron-phonon interactions (see e.g. Ref. 198). As a consequence the SNS system can undergo fluctuations between a ground state and an excited state. The situation encountered here exhibits a close analogy to the low-lying excitations in a normal metal ring penetrated by an Aharonov-Bohm loop. If the ring is closed, the excitation away from the ground state has to be described in a canonical ensemble. If the ring is coupled via a side branch to an electron reservoir, carrier exchange is permitted, and the discussion has, as in the problem at hand, to be carried out in the grandcanonical ensemble 199.200]. At equilibrium the occupation probability of the two states is $f_{+}=f\left(\epsilon_{+}(\phi)\right)$ and $f_{-}=f\left(\epsilon_{-}(\phi)\right)$, where $f$ is the Fermi distribution with energy measured away from the center of the gap of the superconductor. Note that $f_{-}=1-f_{+}$. To investigate the dynamics of this system, in the presence of a bath permitting inelastic transitions, we investigate the relaxation of the non-equilibrium distribution $\rho_{ \pm}$towards the instantaneous equilibrium distribution function with the help of the Debye-Boltzmann-like equations

$$
d \rho_{ \pm} / d t=-\gamma\left(\rho_{ \pm}-f_{ \pm}(t)\right) .
$$

If the system is driven out of equilibrium, the instantaneous distribution function is time-dependent. Eq. (180) states that the non-equilibrium distribution tries to follow the instantaneous distribution but can do that at best with a time lag determined by $\gamma^{-1}$. The timedependent readjustment of the distribution $\rho$ is achieved with inelastic processes and is thus dissipative. To find the resulting noise we investigate the response of the current to a small oscillating phase $\delta \phi(\omega) e^{i \omega t}$ superimposed on the dc phase. The current is $I=$ $-(e / \hbar)\left[\left(d \epsilon_{+} / d \phi\right) \rho_{+}+\left(d \epsilon_{-} / d \phi\right) \rho_{-}\right]$. The Josephson relation, $d \phi / d t=-(2 e / \hbar) V$, leads to a conductance which in the zero frequency limit is given by [200]

$$
G(\phi)=\left.\left(\frac{2 e^{2}}{\gamma \hbar^{2}}\right)\left(\frac{d \epsilon}{d \phi}\right)^{2}\left(-\frac{d f}{d \epsilon}\right)\right|_{\epsilon=\epsilon_{+}} .
$$

The resulting thermal noise of the Josephson current follows from the fluctuation dissipation theorem $S=$ $4 k_{B} T G$ and, as found by Averin and Imam [201] and Martín-Rodero, Levy Yeyati, and García-Vidal [202], is given by

$$
S=\frac{2}{\gamma(\phi)}\left(\frac{e \Delta}{\hbar} \frac{\sin (\phi / 2)}{\cosh \left(\epsilon_{0} / 2 k_{B} T\right)}\right)^{2} .
$$

The peculiar feature of both the conductance (181) and the noise (182) is their divergence as the damping $\gamma$ tends to zero. Furthermore, since $(-d f / d \epsilon)$ in Eq. (181) is proportional to $1 / k_{B} T$, the Nyquist noise given by Eq. (182) is not proportional to $k_{B} T$, as in open systems. Since in the zero temperature limit $\gamma$ can be expected to tend rapidly to zero, the Nyquist noise may actually grow as the temperature is lowered.

Instead of a small amplitude ac oscillation of the phase, we can also consider a phase that linearly increases with time, $\phi=2 \mathrm{eVt} / \hbar$. In a junction without dissipation, we now have an ac Josephson effect. In a system which permits inelastic transitions, the ac Josephson current will be accompanied by a dissipative current. If the induced voltage is small, we can to linear order in $V$ again determine the conductance $G_{J}$, where the index $J$ is to remind us that this conductance occurs in parallel with the ac Josephson effect. Note that the two energy bands $\epsilon_{ \pm}$cross at $\phi= \pm \pi$. To describe this crossing, we extend the range of $\phi$ from $-2 \pi$ to $2 \pi$. Ref. [200] finds that $G_{J}$ and $G$, as given by Eq. (181), are related,

$$
G_{J}=\frac{1}{4 \pi} \int_{-2 \pi}^{2 \pi} d \phi G(\phi)=\frac{e^{2}}{2 \pi \gamma \hbar^{2}} \int_{-2 \pi}^{2 \pi}\left(\frac{d^{2} \epsilon_{+}}{d \phi^{2}}\right) f_{+}(\phi) d \phi .
$$

Thus, $G_{J}$ is inversely proportional to the "effective mass" (weighted by the equilibrium distribution function). We could of course derive this result directly from Eq. (180). In the zero temperature limit the effective mass is $1 / m^{*}=2 \Delta / \pi$, and the conductance $G_{J}$ is finite and given by $G_{J}=2 e^{2} \Delta / \pi \gamma \hbar^{2}$. From the fluctuation dissipation theorem, we obtain an equilibrium noise

$$
S=\frac{4 e^{2} \Delta k_{B} T}{\pi \hbar^{2} \gamma}
$$

which (unlike Eq. (182)) is proportional to the temperature. Eq. (184) was obtained by Averin and Imam 201] and Cuevas, Martín-Rodero, and Levy Yeyati [203].

Non-equilibrium noise. For larger voltages but still $e V \ll \Delta(V>0)$, the average dc current becomes a nonlinear function of voltage. The current peaks for $\mathrm{eV} \sim \hbar \gamma$ when dissipation due to the mechanism described above is maximal. We can no longer invoke the fluctuationdissipation theorem to find the noise. Instead, a direct calculation of the current-current correlation function $\left\langle\hat{I}\left(t_{1}\right) \hat{I}\left(t_{2}\right)+\hat{I}\left(t_{2}\right) \hat{I}\left(t_{1}\right)\right\rangle$ is needed. Since the current oscillates with frequency $2 \mathrm{eV} / \hbar$ and its harmonics, the correlation function depends not only on the time difference $t-t^{\prime}$, as for noise away from stationary states, but also periodically on the total time $t=\left(t_{1}+t_{2}\right) / 2$. Averin and Imam 201, and Cuevas, Martín-Rodero, and Levy Yeyati 203 used the Green's function technique to obtain results for the noise power $S(\omega)$ which is this correlation function averaged over $t$ and Fourier-transformed 
with respect to $t_{1}-t_{2}$. For the discussion of this far from equilibrium noise, we refer the reader to Refs. [201,203. For low voltages $e V \ll \hbar \gamma$, and for an energy independent damping constant $\gamma$, the calculation reproduces Eq. (184), whereas for $\hbar \gamma \ll e V \ll \Delta$ the noise actually decreases with voltage

$$
S=\left(3-\frac{8}{\pi}\right) \frac{\gamma \Delta^{2}}{\hbar V^{2}}
$$

The voltage dependence of the noise is quite unusual in this case, and exhibits a peak for $e V \sim \hbar \gamma$.

The result (185) has the following interpretation. The mechanism of charge transport in SNS structures for $e V \ll \Delta$ is multiple Andreev reflections (MARs) [204]. Imagine an electron with energy $E, \Delta<E<\Delta+\mathrm{eV}$ (measured from the chemical potential of the right contact), emanating from the right contact. During the motion in the normal region it loses the energy $\mathrm{eV}$, and thus when it arrives at the left superconducting bank it has an energy below $\Delta$. This electron may only be Andreev reflected and converted into a hole, which (due to the opposite sign of the charge) loses the energy $\mathrm{eV}$ again. The hole is again Andreev reflected at the right interface, and this process goes on ${ }^{4}$, until the energy of the initial electron falls below $-\Delta$. The number of these MARs is equal to $2 \Delta / \mathrm{eV}$. In each individual Andreev reflection the charge $2 e$ is transferred to or from the condensate, and to avoid double counting, we must only take the reflections happening at the same interface. Therefore the whole MAR process is accompanied by a transfer of charge $2 \Delta / V$ (for $e V \ll \Delta$ ). In view of this, the noise (185) may be interpreted [201] as "shot noise" of the $2 \Delta / \mathrm{eV} \gg 1$ charge quanta. In this sense this noise is giant: it greatly exceeds the Poisson value $2 e I$. The general expression for noise can be written explicitly as a sum of contributions of Andreev reflections of different orders 201,203.

Now we briefly discuss the case of a non-ideal contact, i.e. when the two electrodes are separated by a barrier of arbitrary transparency. First, for a finite but small reflection coefficient $1-T$ an additional source of noise is given by Landau-Zener transitions between the two subgap states, as discussed by Averin [205]. The probability of these transitions, which exist even at zero temperature, is $\nu=\exp (-\pi(1-T) \Delta / e V)$, and noise is caused by the randomness of these transitions. Naveh and Averin 206] obtained the following result for the noise due to this mechanism,

\footnotetext{
${ }^{43}$ This expression is not explicit in Ref. 201, but can be easily derived in the limit of strictly zero temperature.

${ }^{44} \mathrm{MAR}$ is a fully coherent process. It cannot take place, for instance, if the length of the junction is longer than the phase breaking length. In this limit the systems acts rather as two independent NS interfaces. Another limitation is $e V \gg \hbar \gamma$.
}

$$
S=\frac{8 e \Delta^{2}}{\pi \hbar V} \nu(1-\nu)
$$

They also considered the generalization to the multichannel case and analyzed a structure with a normal diffusive conductor between the two superconductors. Taking into account the distribution of transmission eigenvalues (87) of a normal conductor yields 206.

$$
S=\frac{(2 \Delta)^{3 / 2}}{(e V)^{1 / 2}} G(\sqrt{2}-1),
$$

where $G$ is the Drude conductance of the normal region. The noise diverges for low voltages as $V^{-1 / 2}$.

If the transparency of the barrier is low, we return to the case of a classical Josephson junction. The amplitude of a MAR process containing $n$ Andreev reflections is proportional to $T^{n}$, where $T$ is the transmission probability of the junction. Thus, for the classical case MAR's are totally suppressed. The case of arbitrary transparency was investigated by Cuevas, Martín-Rodero and Levy Yeyati 203], who described the crossover between these two regimes.

Bezuglyi et al [207] considered a tunnel barrier (an insulating layer) inserted in the middle of a long SNS constriction. This geometry is different from the standard Josephson junction problem. Instead, bound Andreev states (similar to what has been discussed before for the NS interface with a barrier) are formed in both parts of the normal region, separated by the insulating layer. An electron in the left part, before being converted to a hole at the left NS interface, is oscillating many times before it tunnels (as an electron or a hole) through the barrier, and starts oscillating again. This picture resembles 207 transport in a diffusive metallic wire, which gives us a hint that shot noise may be suppressed in comparison with its "giant Poisson" value, i.e. the value corresponding to the effective charge $2 \Delta / V$. Indeed, Ref. 207 finds that in the limit of low voltages the effective charge is $2 \Delta / 3 \mathrm{~V}$, which surprisingly reminds us of the $1 / 3$-suppression of shot noise in metallic diffusive wires.

For high voltages $\mathrm{eV} \gg \Delta$ imperfect Andreev reflections lead to the saturation of noise, similar to NS structures. For an ideal junction the saturation value $S=8 e^{2} \Delta /(15 \pi \hbar)$, found by Hessling et al 208, is two times as large as for an ideal NS interface (167). For a non-ideal contact, shot noise in this regime equals its normal state value $S=2 e\langle I\rangle$; there is also a voltage independent contribution (excess noise) [203,206. The origin of this excess noise are MAR processes, and the physics is similar to that encountered in the discussion of excess current (see e.g. Ref. 204]).

Experiments. Recently a number of efforts have been made to observe the giant shot noise, caused by multiple Andreev reflections. Experiments by Misaki, Saito, and Hamasaki [209], and Misaki et al [210] used a sandwich of $\mathrm{Nb}$ and $\mathrm{NbN}$ superconducting films, separated by a point contact. However, the giant shot noise was 
not observed in these experiments, possibly because of additional scattering inside the point contact.

An experimental observation of MAR-enhanced shot noise is reported by Dieleman et al 211. They investigate noise in a $\mathrm{NbN} / \mathrm{MgO} / \mathrm{NbN}$ structure, where the two superconducting layers $(\mathrm{NbN})$ are separated by an insulator. The main feature observed in this experiment was a decrease of the Fano factor (which is theoretically predicted to be $2 \Delta / e V$ for $e V \ll \Delta)$ for voltages up to $2 \Delta$. For voltages $e V \sim 2 \Delta / n, n \in \mathcal{Z}$, a step-like structure is observed (Fig. 29).

Hoss et al 212 carried out measurements on $\mathrm{Nb} / \mathrm{Au} / \mathrm{Nb}, \mathrm{Al} / \mathrm{Au} / \mathrm{Al}$, and $\mathrm{Al} / \mathrm{Cu} / \mathrm{Al}$ junctions, where the $\mathrm{Au}$ and $\mathrm{Cu}$ layers were essentially diffusive conductors. They observe a well pronounced peak in the voltage dependence of the shot noise for low voltages (much less than $\Delta / e)$. In addition, they also plot the Fano factor, which turns out to be linear in $V^{-1}$ in the whole range of voltages, but with the coefficient higher than $2 \Delta / e$. This discrepancy with theory is not understood.

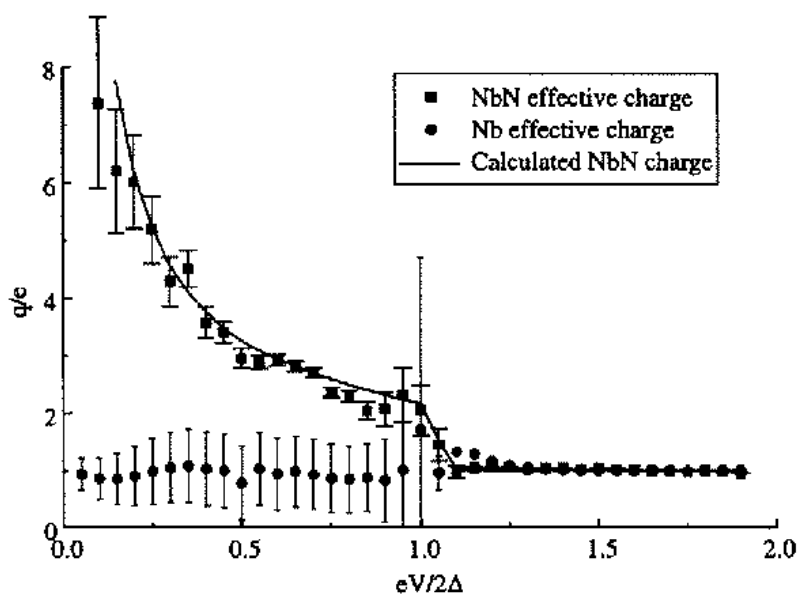

FIG. 29. Experimental results of Dieleman et al 211. The Fano factor (plotted here as an effective charge, black squares) is compared with the theoretical prediction (solid line).

In the experiments by Jehl et al 181 on long $\mathrm{Nb} / \mathrm{Al} / \mathrm{Nb} \mathrm{SNS}$ contacts, the two NS interfaces act effectively independently, and MAR processes are suppressed. Thus, the physics of this experiment resembles more that of a single NS interface, as we have discussed above.

\section{LANGEVIN AND MASTER EQUATION APPROACH TO NOISE: DOUBLE-BARRIER STRUCTURES}

\section{A. Quantum-mechanical versus classical theories of shot noise}

In this and the next Section, we consider classical theories of shot noise in various systems. By doing this, we leave the main road that started from basic quantum mechanics and, through a number of exact transformations and well-justified approximations, lead us to the final results for shot noise. In contrast, classical theories are mostly based on the Langevin approach, which has a conceptually much weaker and less transparent foundation. Indeed, the Langevin equation is equivalent to the Fokker-Planck equation under the condition that the random Langevin sources are Gaussian distributed 45 (see e.g. Ref. 213). In turn, the Fokker-Planck equation is derived from the master equation in the diffusion approximation, and this procedure determines the pair correlation function of Langevin sources. In practice, however, such a basic derivation is usually not presented. The correlation function is written based on some ad hoc considerations rather than derived rigorously. For doublebarrier structures, which are considered in this Section, many results have been derived directly from the master equation, and thus are far better justified than many discussions for other structures. The next Section is devoted to the Boltzmann-Langevin approach in disordered conductors, and, to our knowledge, no attempt to obtain the final results from the master equation, or to justify microscopically the starting Boltzmann equation with Langevin sources, has ever been performed 16 .

The reality, though it may be surprising to some readers, is that in all available cases when the results of classical calculations of shot noise can be compared to exact quantum results averaged over an ensemble, based on the scattering or Green's functions approaches, they turn out to be identical. It is the fact that for many systems the ensemble averaged quantities are classical which makes classical Boltzmann-Langevin theories of shot noise in mesoscopic conductors credible even in those situations, where quantum results are not available. The fact that the ensemble averaged quantum result and the classical result agree is best illustrated by considering for a moment a metallic diffusive wire. A calculation of the conductance can be performed purely quantum-mechanically by finding the scattering matrix computationally or using random matrix theory. After ensemble averaging the leading order result for the conductance is just the Drude result for the conductance of the wire which we can find by solving a diffusion equation. This situation persists

\footnotetext{
${ }^{45}$ Given the results for the distribution of transmitted charge (Appendix A), it is apparent that the Langevin sources are not Gaussian distributed. Possibly, this does not affect the shot noise, which is related to the second cumulant of the Langevin sources.

${ }^{46}$ Quite recently Nagaev 214 has shown that the zero frequency results for shot noise in metallic diffusive wires which are obtained in a quantum-mechanical Green's functions technique, are equivalent to those available from the BoltzmannLangevin approach, even if the interactions are taken into account. This is a considerable step forward, but it still does not explain why the Boltzmann-Langevin approach works.
} 
if we consider the noise: the leading order of the noise, the $1 / 3$-suppression of shot noise in a metallic diffusive wire can be found by ensemble averaging a quantummechanical calculation 74 and/or from a classical consideration [75]. If the two procedures would not agree to leading order, it would imply a gigantic quantum effect. Of course, effects which are genuinely quantum, like the Aharonov-Bohm effect, weak localization, or the quantum Hall effect, cannot be described classically. This consideration also indicates the situations where we can expect differences between a quantum approach and a classical approach: Whenever the leading order effect is of the same order as the quantum corrections we can obviously not find a meaningful classical description.

The Langevin approach essentially takes the Poissonian incoming stream of particles and represents it as a random fluctuating force acting even inside the system. In the language of the scattering approach, this would mean that the Poissonian noise of the input stream is converted into the partition noise of the output stream. Another possible classical approach to the shot noise would be to take the Poissonian input stream as a sequence of random events, and to obtain the distribution of the carriers in the output stream after the scattering events took place. To our knowledge, this approach has not been realized precisely in this form. Landauer 215] discusses noise in diffusive metallic conductors from a similar point of view, but does not calculate the distribution of outgoing particles. Chen et al [216] attempt to modify the distribution function in ballistic systems to take into account the Pauli principle, assuming that the time the particle spends inside the system is finite. Barkai, Eisenberg, and Schuss [217] and van Kampen [218] consider the case when the electrons may arrive from two reservoirs, and are transmitted or reflected with certain probabilities. The Pauli principle forbids two electrons to be in the channel simultaneously.

Though there is no doubt that this approach, if realized, would yield the same value of the shot noise as more powerful methods, it would still help to visualize the results and it might allow a simple generalizations to the interacting systems. Raikh 219] and Imamoglu and Yamamoto [220] have suggested a generalization to the Coulomb blockade regime. Raikh [219] shows how the noise in the Coulomb blockade regime may be expressed if the transformation from the Poisson input stream to the correlated output stream is known for non-interacting electrons. Imamoḡlu and Yamamoto [220] assume that the Poisson distribution is modified in some particular way by the finite charging energy, and are able to obtain sub-Poissonian shot noise suppression. We treat shot noise in the Coulomb blockade regime later on (Section VII) by more elaborate methods.

\section{B. Suppression of shot noise in double-barrier structures}

We consider now transport through quantum wells, which were described quantum-mechanically in Section II. The tunneling rates through the left $\Gamma_{L}$ and the right $\Gamma_{R}$ barrier are assumed to be much lower than all other characteristic energies, including temperature ${ }^{17}$. Introducing the distribution function in the well $f_{w}(E)$, we write the charge of the well $Q_{w}$ in the form

$$
Q_{w}=e \nu_{2} \mathcal{A} \sum_{n} \int_{0}^{\infty} d E_{z} d E_{\perp} f_{w}\left(E_{z}+E_{\perp}\right) \delta\left(E_{z}-E_{n}^{r}\right),
$$

where the energy $E_{z}$ in the well is counted from the band bottom $e U$ in the well ${ }^{48}$ (Fig. 8), and the sum is taken over all the resonant levels.

Now we introduce the charges $Q_{L}(t)$ and $Q_{R}(t)$ which have passed through the left and right barriers, respectively, from the time $t=-\infty$ until the time $t$. At any instant of time the charge of the well is $Q_{w}(t)=$ $Q_{L}(t)-Q_{R}(t)$. The time evolution of the charge $Q_{L}$ is determined by the rate equation,

$$
\dot{Q}_{L}=e\left(\gamma_{L \rightarrow}-\gamma_{L \leftarrow}\right),
$$

where $\gamma_{L \rightarrow}$ and $\gamma_{L \leftarrow}$ are transition rates through the left barrier, from the reservoir to the well and from the well to the reservoir, respectively. We have

$$
\begin{aligned}
& \gamma_{L \rightarrow}=\frac{\nu_{2} \mathcal{A}}{\hbar} \sum_{n} \int_{e(V-U)}^{\infty} d E_{z} \int_{0}^{\infty} d E_{\perp} \Gamma_{L n} \delta\left(E_{z}-E_{n}^{r}\right) \\
& \times f_{L}\left(E_{z}+E_{\perp}+e U-e V\right)\left[1-f_{w}\left(E_{z}+E_{\perp}\right)\right], \\
& \gamma_{L \leftarrow}=\frac{\nu_{2} \mathcal{A}}{\hbar} \sum_{n} \int_{e(V-U)}^{\infty} d E_{z} \int_{0}^{\infty} d E_{\perp} \Gamma_{L n} \delta\left(E_{z}-E_{n}^{r}\right) \\
& \times\left[1-f_{L}\left(E_{z}+E_{\perp}+e U-e V\right)\right] f_{w}\left(E_{z}+E_{\perp}\right) .
\end{aligned}
$$

Here we wrote the distribution function of the left (right) reservoir in such a way that the energy is counted from the chemical potential of the left (right) reservoir. Similarly, $\dot{Q}_{R}=e\left(\gamma_{R \rightarrow}-\gamma_{R \leftarrow}\right)$ is expressed in terms of the transition rates through the right barrier, which are written analogously to Eq. (188).

Further progress is easy in two cases: either the tunneling rates $\Gamma_{L, R n}$ do not depend on $n$ (and equal $\Gamma_{L, R}$ ), or there is only one resonant level of the longitudinal motion

\footnotetext{
${ }^{47}$ In terms of the quantum-mechanical derivation, this would mean that the transmission coefficient (75) is replaced by $T(E)=2 \pi \Gamma_{L n} \Gamma_{R n} \Gamma_{n}^{-1} \delta\left(E-E_{n}^{r}\right)$.

${ }^{48}$ Note that the notations here and below differ from those introduced in Section III: All the energies are measured from the corresponding band bottoms.
} 
in the relevant range of energies. Taking into account Eq. (186), we obtain

$$
\begin{gathered}
\dot{Q}_{L}=I_{L}-\hbar^{-1} \Gamma_{L}\left(Q_{L}-Q_{R}\right), \\
\dot{Q}_{R}=I_{R}+\hbar^{-1} \Gamma_{R}\left(Q_{L}-Q_{R}\right),
\end{gathered}
$$

where we have introduced

$$
\begin{aligned}
I_{L} & =\frac{e \nu_{2} \mathcal{A}}{\hbar} \Gamma_{L} \sum_{n} \int_{e(V-U)}^{\infty} d E_{z} \\
& \times \int_{0}^{\infty} d E_{\perp} \delta\left(E_{z}-E_{n}^{r}\right) f_{L}\left(E_{z}+E_{\perp}+e U-e V\right), \\
I_{R} & =-\frac{e \nu_{2} \mathcal{A}}{\hbar} \Gamma_{L} \sum_{n} \int_{0}^{\infty} d E_{z} \\
& \times \int_{0}^{\infty} d E_{\perp} \delta\left(E_{z}-E_{n}^{r}\right) f_{R}\left(E_{z}+E_{\perp}+e U\right) .
\end{aligned}
$$

Note that we derived the rate equations (189) without specifying the distribution function $f_{w}$. Thus, within the approximations used here, the rate equations are the same, independently of the relaxation rate in the well (which determines the distribution $f_{w}$ ).

The current, for instance, through the left barrier, is given by the sum of the particle current $I_{L p}=\dot{Q}_{L}$ and the displacement current $I_{L d}=c_{L} \dot{Q}_{w} /\left(c_{L}+c_{R}\right)$, where we have introduced the capacitances $c_{L}$ and $c_{R}$ of the left and the right barrier, respectively. Using this gives for the total current

$$
I=\frac{c_{L} \dot{Q}_{R}+c_{R} \dot{Q}_{L}}{c_{L}+c_{R}}
$$

which is often cited as Ramo-Shockley theorem 19 . The calculation of the current through the right barrier yields the same result, demonstrating that the total current is conserved.

Up to now, we have only discussed average quantities. The idea of the Langevin approach is that the (current) fluctuations can be calculated from the same rate equations (189), if random currents (Langevin sources) are added to their right-hand sides. We write

$$
\begin{gathered}
\dot{Q}_{L}=I_{L}-\hbar^{-1} \Gamma_{L}\left(Q_{L}-Q_{R}\right)+\xi_{L}(t), \\
\dot{Q}_{R}=I_{R}+\hbar^{-1} \Gamma_{R}\left(Q_{L}-Q_{R}\right)+\xi_{R}(t),
\end{gathered}
$$

where the Langevin sources $\xi_{L, R}(t)$ have the following properties. They are zero on average, $\left\langle\xi_{\alpha}(t)\right\rangle=0, \alpha=$

\footnotetext{
${ }^{49}$ Many papers in the field are flawed since they use $I=$ $\left(\dot{Q}_{L}+\dot{Q}_{R}\right) / 2$ and subsequently claim the validity of the results for arbitrary barriers. Similarly, evaluating Eq. (191) with the help of the free electron results for $\dot{Q}_{L}$ and $\dot{Q}_{R}$ leads to a current conserving answer but not to a self-consistent result. Compare Eqs. 195) and 201).
}

$L, R$. Furthermore, they are correlated only for the same barrier, and the correlation function describes Poissonian shot noise at each barrier,

$$
\left\langle\xi_{\alpha}(t) \xi_{\beta}\left(t^{\prime}\right)\right\rangle=e\langle I\rangle \delta\left(t-t^{\prime}\right) \delta_{\alpha \beta},
$$

where $\langle I\rangle$ is the average current. To find the noise power, we do not need to specify higher cumulants of the Langevin sources. As we mentioned above, the definition (193) is intuitive rather than the result of a formal derivation. However, the results we obtain in this way coincide with those found by ensemble averaging the quantummechanical results.

The equations (192) are linear and can be easily solved. The average current is

$$
\langle I\rangle=\frac{\Gamma_{R} I_{L}+\Gamma_{L} I_{R}}{\Gamma},
$$

where again $\Gamma=\Gamma_{L}+\Gamma_{R}$. The finite frequency shot noise power is found to be

$$
\begin{aligned}
S(\omega) & =2 e\langle I\rangle\left[\frac{c_{L}^{2}+c_{R}^{2}}{c^{2}}\right. \\
& \left.+\frac{2}{\Gamma^{2}+(\hbar \omega)^{2}}\left(\Gamma^{2} \frac{c_{L} c_{R}}{c^{2}}-\Gamma_{L} \Gamma_{R}\right)\right],
\end{aligned}
$$

with the definition $c \equiv c_{L}+c_{R}$.

For zero frequency Eq. (195) gives the result (78), $F=\left(\Gamma_{L}^{2}+\Gamma_{R}^{2}\right) / \Gamma^{2}$. For high frequencies $\hbar \omega \gg \Gamma$ we have $S(\omega)=2 e\langle I\rangle\left(c_{L}^{2}+c_{R}^{2}\right) / c^{2}$. This expression can be obtained from Eq. (191) based on the assumption that the particle currents $Q_{L}$ and $\dot{Q}_{R}$ fluctuate independently and according to the Poisson shot noise. The crossover frequency between these regimes is $\omega \sim \Gamma / \hbar$ : In accordance with general expectations, the frequency dependence of the shot noise is governed by time-scales inherent to the conductor. We note finally that for a symmetric double barrier, $c_{L}=c_{R}$ and $\Gamma_{L}=\Gamma_{R}$, the noise power (195) is frequency independent and equal to $S(\omega)=e\langle I\rangle$.

Note also that Eq. (195) does not contain the highfrequency Nyquist noise (129), which is proportional to $\hbar|\omega|:$ Zero-point noise is quantum-mechanical and cannot be reproduced by a classical discussion.

The classical derivation of Eq. (195), based on the master equation approach, was given independently by Chen and Ting 134 $\left(c_{L}=c_{R}\right.$, zero frequency limit) and by Davies et al 54 (general case), and later by Chen 221. $\left(c_{L}=c_{R}\right.$, arbitrary frequency) and Müller et al 222 $(\omega=0)$. Sun and Milburn 223,224] and Milburn 225] developed a quantum master equation approach and also derived the same result (195). The Langevin approach was applied to double-barrier structures in Ref. [226]; here we have given an extended version of the derivation. We also remark that the frequency dependence of the noise of the double-barrier structure (195) was obtained by Runge [57 $\left(c_{L}=c_{R}\right)$ and by Lund $\mathrm{B} \varnothing$ and Galperin [58] (general case) using the non-equilibrium Green's functions method. 
Beenakker and de Jong 89,90 consider the two-barrier suppression using the conceptually similar BoltzmannLangevin approach, described in the Section VI. They also investigate the case of $n$ identical barriers in series and obtain for the Fano factor

$$
F=\frac{1}{3}\left[1+\frac{n(1-T)^{2}(2+T)-T^{3}}{[T+n(1-T)]^{3}}\right],
$$

where $T$ is the transmission coefficient of a barrier. This expression gives $F=1-T$ for $n=1, F=1 / 3$ for $n \rightarrow \infty$ (which mimics a diffusive wire), and reduces to $F=1 / 2$ for $T \ll 1$ for the two-barrier case, $n=2$. Thus, the crossover of the ensemble averaged shot noise between a two-barrier and many-barrier (diffusive) system can be described classically.

The theory we have presented above has a number of drawbacks, which we discuss now. First, the charges $Q_{L}$ and $Q_{R}$ are assumed to be continuous, and thus Coulomb blockade effects cannot be treated in this way (see Section VII). Even the charging effects which exist if charge quantization can be neglected are not properly taken into account. The discussion thus far has neglected to include the response to the fluctuating electric potential in the well. Thus the Ramo-Shockley theorem, as it has been used here, and is applied in much of the literature, leads to a current conserving, but, as we discuss below, not to a self-consistent result for the frequency dependence of the shot noise. In the next subsection we show that charging effects can lead to the enhancement of shot noise above the Poisson value.

Furthermore, our consideration is limited to zero temperature 50, and it is not immediately clear how the Langevin approach should be modified in this case to reproduce correct expressions for the Nyquist noise. Another limitation is that our derivation assumes that the tunneling rates through each of the resonant levels are the same. If this is not the case, the rate equations do not have the simple form (189), but instead start to depend explicitly on the distribution function $f_{w}$. Whereas the above derivation does not require any assumptions on the distribution of the electrons in the well $f_{w}$ (i.e. any information on the inelastic processes inside the well), generally this information is required, and it is not a priori clear whether the result on noise suppression depends on the details of the inelastic scattering.

The last two issues are relatively easily dealt with in the more general master equation approach. Chen and Ting [134], Chen [227, and independently Davies et al [54] solved the master equation in the sequential tunneling limit, when the electrons, due to very strong inelastic scattering inside the well, relax to the equilibrium state. They found the results to be identical to those obtained by quantum-mechanical methods (which require

\footnotetext{
${ }^{50}$ To use the kinetic equation formalism, we had to assume $k_{B} T \gg \Gamma$; on the other hand Eq. (193) states $k_{B} T \ll e V$.
}

quantum coherence, i.e. absence of inelastic scattering), and concluded that inelastic processes do not affect shot noise suppression in quantum wells. Later, Iannaccone, Macucci, and Pellegrini 228] solved the master equation allowing explicitly for arbitrary inelastic scattering, and found that the noise suppression factor is given by Eq. (78) at zero temperature for arbitrary inelastic scattering provided the reservoirs are ideal. They also studied other cases and temperature effects. This picture seems to be consistent with the results obtained quantum-mechanically by attaching voltage probes to the sample (subsection II G).

We note here, however, that there is no consensus in the literature concerning this issue. First, we discuss the results obtained quantum-mechanically by Davies, Carlos Egues, and Wilkins 229. They start from the exact expression (74) and average it over the phase $\phi$, allowing for inelastic scattering (dephasing). Instead of assuming that the phase is a uniformly distributed random variable, they postulated $\left\langle\exp \left(i \phi_{1}+\phi_{2}\right)\right\rangle=$ $\left\langle\exp \left(i \phi_{1}\right)\right\rangle\left\langle\exp \left(i \phi_{2}\right)\right\rangle$. For the Fano factor (at zero frequency) they obtain in this way

$$
F=1-\frac{2 \Gamma_{L} \Gamma_{R}}{\Gamma^{2}}\left(\frac{\Gamma}{\Gamma+\Gamma_{i n} / 2}\right),
$$

where $\Gamma_{i n}$ is the rate of inelastic scattering, proportional to $1-\langle\exp (i \phi)\rangle$. Thus, in their model inelastic scattering enhances the Fano factor, driving noise towards the Poisson value. While it is clear that for certain models of inelastic scattering shot noise is affected by interactions, we do not see a direct relation of the model of Ref. 229] to the voltage probe models which we consider in subsection II G and which yield the result that the Fano factor is interaction insensitive.

Furthermore, Isawa, Matsubara, and Ohuti [230], using the Green's functions approach, find that inelastic processes leading to sequential tunneling affect the Fano factor. Their theory, however, is explicitly not current conserving. We mentioned already the result by Lund $\mathrm{B} \varnothing$ and Galperin [59], who report suppression of the Fano factor by electron-phonon interactions. Their results clearly contradict the conclusions based on the voltage probe models.

A related issue is investigated by Sun and Milburn 223,224, who apply the quantum master equation to the analysis of noise in a double-well (triple-barrier) structure. With this approach, they are able to study the case when the two wells are coupled coherently. Their results show an abundance of regimes depending on the relation between the coupling rates to the reservoirs, elastic rates, and the coupling between the two wells.

To conclude this subsection, we address here one more problem. The result (78) predicts that the noise suppression factor may assume values between $1 / 2$ and 1 , depending on the asymmetry of the double barrier. The question is whether interaction effects, under some circumstances, may lead to Fano factors above 1 (superPoissonian noise) or below $1 / 2$. Relegating the problem 
of super-Poissonian noise to the next subsection, we only discuss here the possible suppression of shot noise below $1 / 2$. Experimentally, noise suppression below $1 / 2$ was observed in early experiments by Brown [231], and recently by Przadka et al [68] and by Kuznetsov et al [232.

Early papers on the subject (Han and Barnes [233], Alam and Khondker [234, Sheng and Chua [235], Jahan and Anwar [236] ) predict shot noise suppression down to zero either with frequency or even at zero-frequency. Following Ref. 233], these works treat current fluctuations as a superposition of density and velocity fluctuations, with a self-consistent treatment of interaction effects. However, apparently they did not include the partition noise $(T(1-T))$ in their consideration. Since it is precisely the partition noise which produces the minimal suppression of $1 / 2$, it is not quite surprising that their theory predicts lower suppression factors.

Sugimura [237] and, independently, Carlos Egues, Hershfield, and Wilkins 238 propose a model in which the states in the well are inelastically coupled to the degrees of freedom of the reservoirs. This model, indeed, yields noise suppression below $1 / 2$ in a limited parameter range. The minimal suppression factor given in Ref. 238] is 0.45 , which is still above the experimental data. This direction of research looks promising, but certainly requires more efforts.

\section{Interaction effects and super-Poissonian noise enhancement}

Prior to the discussion of charging effects, we briefly comment on how quantum wells operate in the strongly non-linear regime $\left(\mathrm{eV} \sim E_{F}\right)$, provided charging effects are not important. For simplicity, we assume that there is only one resonant level of the longitudinal motion $E_{0} \equiv$ $E_{0}^{r}$ in the relevant range of energies, and all others levels lie too high to be of importance, $E_{n}^{r} \gg e V, E_{F}$ for $n>0$. We assume also $E_{0}>E_{F}$, and $V>0$, then electrons from the right reservoir cannot enter the well.

Modifying Eq. (79) to take into account that the band bottoms $\mathrm{eV}$ in the left reservoir and $U$ in the quantum well are now finite, and substituting the transmission coefficient, $T\left(E_{z}\right)=2 \pi \Gamma_{L} \Gamma_{R} \Gamma^{-1} \delta\left(E_{z}-E_{0}\right)$, we obtain for the average current

$$
\langle I\rangle=\frac{e \nu_{2} \mathcal{A}}{\hbar} \frac{\Gamma_{L} \Gamma_{R}}{\Gamma}\left(e V-e U+E_{F}-E_{0}\right),
$$

provided $E_{0}+e U-E_{F}<e V<E_{0}+e U$, and zero otherwise. This dependence, which, of course, could also be obtained classically from the rate equations, is shown in Fig. 30a, solid line. The current drops abruptly to zero for $e V=E_{0}+e U$, which corresponds to the passage of the band bottom of the left reservoir through the resonant level in the well. When the smearing of the resonance due to finite tunneling rates is taken into account (the transmission coefficient is not replaced by a deltafunction), the $I-V$ curve becomes smeared (dashed line in Fig. 30a), and the region of negative differential resistance develops around $e V \sim E_{0}+e U$. This was noted by Tsu and Esaki in their early paper 52], and subsequently observed experimentally in Ref. [53].

Now we consider charging effects. The new ingredient is now that the electrostatic potential in the well $U$ is not an independent parameter any more, but is a function of voltage, which must be calculated self-consistently. Moreover, it has its own dynamics and may fluctuate; we are going to show that the fluctuations of $U$ may considerably enhance noise. Theoretical papers emphasizing the necessity of charging effects for the $I-V$ curve are too numerous to be cited here. For noise, the necessity of a self-consistent treatment in the negative differential resistance region was illustrated in the Green's function approach by Levy Yeyati, Flores, and Anda [239], who, however, did not take into account the fluctuations of $U$. Iannaccone et al [240] suggested that these fluctuations may lead to the enhancement of noise above the Poisson value, and provided numerical results supporting this statement. A self-consistent analytical theory of noise in quantum wells including the fluctuations of the band bottom was developed by the authors 241] in the framework of the scattering approach ${ }^{51}$. The classical theory yields the same results 226]; here we give the classical derivation.

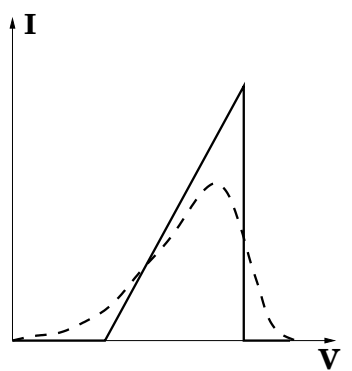

a)

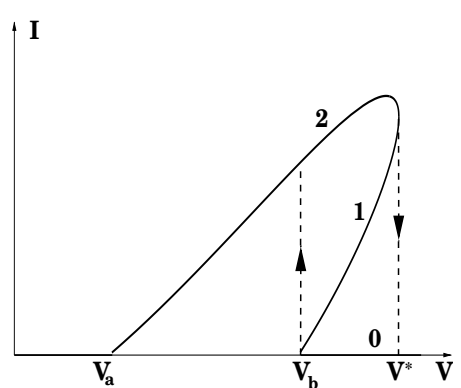

b)
FIG. 30. $I-V$ characteristics of the quantum well: (a) charging effects are neglected; (b) charging effects are taken into account. The values of parameters for (b) are $a_{L}=a_{R}$, $c_{L}=c_{R}, E_{F}=2 E_{0} / 3, e^{2} \nu_{2} \mathcal{A}=10 c_{L}$. For this case $e V^{*}=2.70 E_{0}$.

In the strongly non-linear regime which we discuss here, the energy dependence of the tunneling rates becomes important. To take this into account, we use a simple model and treat each barrier as rectangular. The transmission probability through a rectangular barrier determines the partial decay width of the resonant level,

$$
\begin{aligned}
\Gamma_{L}\left(E_{z}\right) & =a_{L} E_{z}^{1 / 2}\left(E_{z}+e U-e V\right)^{1 / 2} \\
& \times \theta\left(E_{z}\right) \theta\left(E_{z}+e U-e V\right)
\end{aligned}
$$

\footnotetext{
${ }^{51}$ The quantity $U$ serves then as an operator which obeys an operator Poisson equation.
} 


$$
\Gamma_{R}\left(E_{z}\right)=a_{R} E_{z}^{1 / 2}\left(E_{z}+e U\right)^{1 / 2} \theta\left(E_{z}\right) \theta\left(E_{z}+e U\right),
$$

where $a_{L}$ and $a_{R}$ are dimensionless constants (the case of a symmetric well corresponds to $a_{L}=a_{R}$, not to $\Gamma_{L}=$ $\Gamma_{R}$ ). We emphasize that the partial decay widths $\Gamma_{L, R}$ are now functions of $V$ and $U$.

Consider first the average, stationary quantities. Equations (189) are still valid for our case (now $I_{R}=0$, since $E_{0}>\overline{E_{F}}$ ). However, $U$ is no longer an independent variable, but is related to the charge in the well $Q_{L}-Q_{R}$. Assuming that the interaction effects can be described by a charging energy only, we write this relation in the form

$$
\left(Q_{L}-Q_{R}\right)=c_{L}(U-V)+c_{R} U
$$

This equation just states that the total charge of the capacitor equals the sum of charges at the left and the right plates. Equations (189) and (198) must now be solved together, using the expressions for the partial decay widths (197). Combining them, we obtain a closed equation for $U$,

$$
\begin{aligned}
\hbar c \dot{U} & =e \nu_{2} \mathcal{A} \Gamma_{L}\left(e V-e U+E_{F}-E_{0}\right) \\
& \times \theta\left(e V-e U+E_{F}-E_{0}\right) \theta\left(E_{0}+e U-e V\right) \\
& -\left(\Gamma_{L}+\Gamma_{R}\right)\left[c_{L}(U-V)+c_{R} U\right]
\end{aligned}
$$

where $\Gamma_{L, R} \equiv \Gamma_{L, R}\left(E_{0}\right)$.

We analyze now the stationary solutions 52 of Eq. (199). For $0<e V<e V_{a} \equiv c\left(E_{0}-E_{F}\right) / c_{R}$ the only solution is $U=U_{0} \equiv c_{L} V / c$, which corresponds to the charge neutral well (see Eq. (198)): The resonant level is pushed up too high to allow any charge in the well. At $V=V_{a}$ the resonant level passes through the Fermi level of the left reservoir, and with a further increase of voltage, the well is charged, $U>U_{0}$. For $e V_{a}<e V<e V_{b} \equiv c E_{0} / c_{R}$, Eq. (199) has only one stationary solution; however, for $V>\overline{V_{b}}$ three solutions develop. One of them is $U=U_{0}$ and corresponds to the charge neutral well; two other solutions $U_{1}<U_{2}$ describe the charged well. It can be seen from Eq. (199) that the solution $U_{1}$ is unstable, while $U_{0}$ and $U_{2}$ are stable. As the voltage $V$ grows, the solutions $U_{1}$ and $U_{2}$ move towards each other, and at the voltage $V^{*}$ (which is referred below as the instability threshold) merge. For higher voltages $V>V^{*}$, the only stationary solution is $U_{0}$ : The well is charge neutral again, since the resonant level lies too low.

Thus, the new feature due to charging effects is the multi-stability of the system in the range of voltages between $V_{b}$ and $V^{*}$. The $I-V$ characteristics of the quantum well with charging are shown in Fig. $30 \mathrm{~b}$; in the

\footnotetext{
${ }^{52}$ Analytic expressions for the stationary solutions may be obtained, since they obey a cubic equation. However, these expressions are to cumbersome and not very transparent. Instead, we have chosen to give a qualitative discussion, illustrating it by numerical results.
}

multi-stability range the marks 0,1 , and 2 refer to the solutions $U_{0}, U_{1}$, and $U_{2}$, respectively. The current is only non-zero if the well is charged. The instability is manifest in the hysteretic behavior shown by dashed lines in Fig. 30 b: when the voltage is increasing, the well stays charged (solution $U_{2}$ ) until $V^{*}$, and then jumps to the zero-current state $\left(U_{0}\right)$; if the voltage is decreasing, the current is zero until $V_{b}$, and then the jump to the charged state $\left(U_{2}\right)$ happens. This hysteresis was apparently observed experimentally [242]. A finite value of the tunneling rates smears all these features, causing a finite current for all values of voltage. Furthermore, the instability range shrinks, and a region of negative differential resistance appears close to the instability threshold $V^{*}$. For $\Gamma \sim\left(V^{*}-V_{b}\right)$ the instability disappears, and the $I-V$ characteristics resemble the dashed curve in Fig. 30a: The multi-stable regime is only pronounced for wells formed with high tunnel barriers.

Now we turn to the calculation of noise. The principal difficulty which we encounter for the charged well is the following. Equations (189) are now non-linear, since they depend on the potential in the well $U$ in a non-linear way, and $U$, in turn, is related to the charges $Q_{L}$ and $Q_{R}$ via Eq. (199). The Langevin sources, however, can only be added to linear equations 213]. Thus, we have to linearize our set of equations ${ }^{53}$. Restricting ourselves to the voltage range $V_{a}<V<V^{*}$, we write for the potential in the well

$$
U(t)=U_{2}+\Delta U(t)
$$

where $U_{2}(V)$ is a stable stationary solution corresponding to the charged well, and $\Delta U(t)$ are fluctuations. Expanding in $\Delta U$ and adding the Langevin sources with the same properties as before to the resulting linear equations, we write

$$
\begin{aligned}
& \dot{Q}_{L}=\langle I\rangle+\frac{1}{\hbar c}\left[\hbar J-\Gamma_{L}\left(c+c_{0}\right)\right]\left(Q_{L}-Q_{R}\right)+\xi_{L}(t) ; \\
& \dot{Q}_{R}=\langle I\rangle+\frac{1}{\hbar c}\left[\hbar J+\Gamma_{R}\left(c+c_{0}\right)\right]\left(Q_{L}-Q_{R}\right)+\xi_{R}(t),
\end{aligned}
$$

where the $U$-dependent tunneling rates $\Gamma_{L, R}$ are evaluated for $U=U_{2}$, and the quantities $c_{0}=-\partial \bar{Q} / \partial U$ and $J=\partial\left(\Gamma_{R} \bar{Q}\right) / \hbar \partial U$ (also taken for $U=U_{2} ; \bar{Q} \equiv$ $e \nu_{2} \mathcal{A}\left(e V-e U+E_{F}-E_{0}\right) \Gamma_{L} / \Gamma$ is the average charge of the well) are the response of the average charge and current to the increment of the potential in the well. The average current in Eq. (200) is $\langle I\rangle=\Gamma_{R} \bar{Q} / \hbar$.

The noise spectrum, which follows from Eqs. 200, is

$$
S(\omega)=2 e\langle I\rangle\left\{\frac{c_{L}^{2}+c_{R}^{2}}{c^{2}}+\frac{2}{c^{2} \hbar^{2} \omega^{2}+\Gamma^{2}\left(c+c_{0}\right)^{2}}\right.
$$

\footnotetext{
${ }^{53}$ The quantum-mechanical theory [241] also has been developed in linear approximation.
} 


$$
\begin{aligned}
& \times\left[\left(c+c_{0}\right)^{2}\left[-\Gamma_{L} \Gamma_{R}+\frac{c_{L} c_{R} \Gamma^{2}}{c^{2}}\right]\right. \\
& \left.\left.-\hbar J\left(c+c_{0}\right)\left(\Gamma_{L}-\Gamma_{R}\right)+\hbar^{2} J^{2}\right]\right\} .
\end{aligned}
$$

The noise power (201) is strongly voltage dependent via the quantities $c_{0}, J$, and $\Gamma$. In particular, for $V \rightarrow V^{*}$ the combination $c+c_{0}$ tends to zero, as it is seen from Eq. (199). If the charging effects do not play a role, we may set $c_{0}=J=0$ and recover the result (195).

The frequency structure of Eq. (201) is identical to that of Eq. (195); the crossover frequency is $\hbar \omega \sim$ $\Gamma\left(c+c_{0}\right) / c$, and drops down to zero for $V=V^{*}$. For high frequencies the usual result $S=2 e\langle I\rangle\left(c_{l}^{2}+c_{R}^{2}\right) / c^{2}$ is recovered. For zero frequency, we obtain a voltage dependent Fano factor,

$$
F=\frac{1}{2}+2 \frac{(\Lambda-\Delta \Gamma)^{2}}{\Gamma^{2}}, \quad \Delta \Gamma \equiv\left(\Gamma_{L}-\Gamma_{R}\right) / 2 .
$$

We have introduced the "interaction energy"

$$
\Lambda \equiv \frac{\hbar J}{c_{L}+c_{R}+c_{0}}
$$

which has the form of a dimensionless conductance $\hbar J / e^{2}$ multiplied by an effective charging energy of the well $e^{2} /\left(c_{L}+c_{R}+c_{0}\right)$. This quantity contains the relevant information about the charging effects of the well. Eq. (201) is a self-consistent result in contrast to Eq. (195) found by inserting the free electron currents into the Ramo-Shockley formula. We reproduce this result for $\Lambda=0$. We re-emphasize that a calculation of ac conductance or noise using the free-electron results for the currents and the Ramo-Shockley formula is not in general a sound procedure: It assumes that the self-consistent contribution to the currents arising from the internal potential oscillations or fluctuations can be neglected.

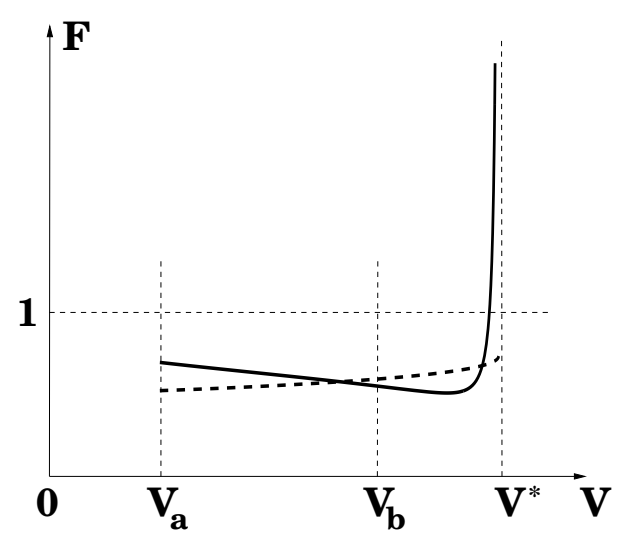

FIG. 31. Voltage dependence of the Fano factor (202) for the same set of parameters as Fig. 30 (solid line); Fano factor (78) for a charge neutral quantum well (dashed line).

For $V \rightarrow V^{*}$ the denominator $c+c_{0}$ of the interaction energy $\Lambda(V)$ quite generally diverges as $\left(V^{*}-V\right)^{-1 / 2}$, while the numerator $J$ stays finite. Thus, the Fano factor diverges according to $\left(V^{*}-V\right)^{-1}$. In particular, close enough to the instability threshold $V^{*}$ the Fano factor increases above one: The noise becomes super-Poissonian. At the onset of current, for $V=V_{a}$ the Fano factor can be calculated in closed form, and one has $1 / 2<F\left(V_{a}\right)<1$. Thus, we describe the transition from sub-Poissonian to super-Poissonian noise. The minimal possible value of the Fano factor in this theory is $F=1 / 2$. It can be shown that the voltage dependence of the Fano factor is peculiar, and, depending on the relative value of the charging effects $e^{2} \nu_{2} \mathcal{A} / c$, noise may be either suppressed as compared with the non-interacting value (78) for low voltages and enhanced for high voltages ("weak interaction scenario"), or enhanced, then suppressed, and only after that enhanced again ("strong interaction scenario"). For details, see Ref. [241]. The voltage dependence of the Fano factor is illustrated in Fig. 31. The divergence of noise is clearly seen.

The finite value of the partial decay widths (tunneling rates) smears the singularities, and, in particular, induces a finite value of the Fano factor for $V=V^{*}$. Another source of deviations from the experimental results is our linearization procedure. For voltages close to the instability threshold the linear approximation is clearly insufficient, and large fluctuations (transitions between the state $U_{2}$ and the state $U_{0}$ ) must be taken into account. These fluctuations also would induce a finite value of noise for $V=V^{*}$. We do not see any reason, however, why these processes should suppress noise below the Poisson value in the whole range of voltages.

Now we briefly review various predictions of the possibility of super-Poissonian noise enhancement in quantum wells. Brown 231] theoretically predicted that noise can be enhanced above the Poissonian value because the energy dependence of transmission probabilities will be affected by the applied voltage in a non-linear way. He, however, did not include partition noise in his consideration, so that for the energy independent transmission probabilities noise is fully Poissonian. Jahan and Anwar [236], who also found super-Poissonian noise enhancement, included self-consistent effect at the level of the stationary transmission probabilities, but also did not take partition noise into account. As we already mentioned, an explanation of the super-Poissonian noise in terms of the potential fluctuations was given by Iannaccone et al 240], and the analytical theory of this enhancement, identifying the relevant energy scales, was proposed in Refs. 241,226.

Experimentally, enhancement of noise in quantum wells, as the voltage approaches the range of negative differential resistance, was observed already in the early experiments by Li et al [64] and by Brown [231]. The super-Poissonian shot noise in the negative differential resistance range was observed by Iannaccone et al [240]. Kuznetsov et al 232] have presented a detailed investigation of the noise oscillations from sub-Poissonian to super-Poissonian values of a resonant quantum well in 
the presence of a parallel magnetic field. The magnetic field leads to multiple voltage ranges of negative differential resistance and permits a clear demonstration of the effect. Their results are shown in Fig. 32 .

To conclude this Section, we discuss the following issue. To obtain the super-Poissonian noise enhancement, we needed multi-stable behavior of the $I-V$ curve; in turn, the multi-stability in quantum wells is induced by charging effects. It is easy to see, however, that the charging (or, generally, interaction) effects are not required to cause the multi-stability. Thus, if instead of a voltage controlled experiment, we discuss a current controlled experiment, the $I-V$ characteristics for the uncharged quantum well (Fig. 30) are multi-stable for any external current. For the case of an arbitrary load line there typically exists a finite range of external parameters where multi-stable behavior is developed. Furthermore, the quantum wells are not the only systems with multistability; as one well-known example we mention Esaki diodes, where the multi-stability is caused by the structure of the energy bands [243].

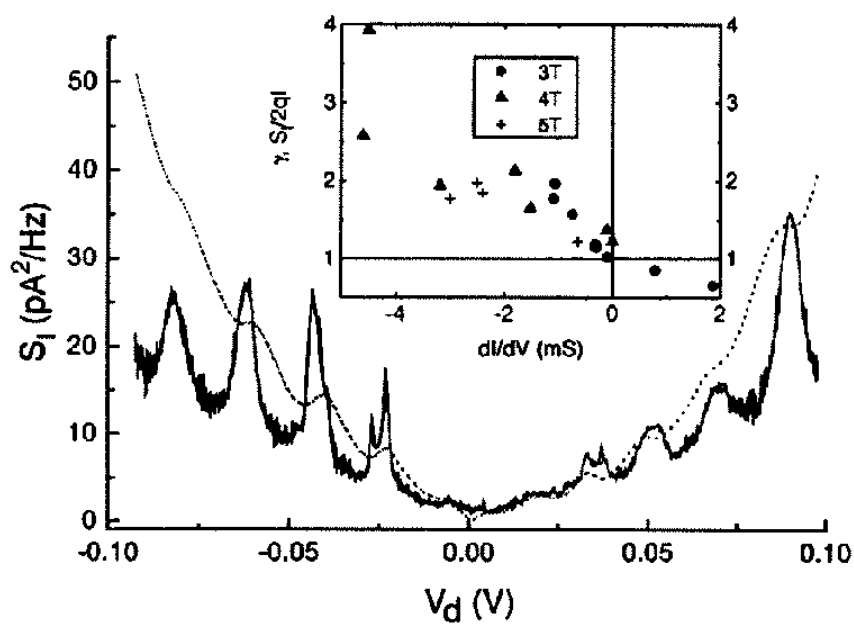

FIG. 32. Experimental results of Kuznetsov et al 232 which show noise in resonant quantum wells in parallel magnetic field. A dotted line represents the Poisson value. It is clearly seen that for certain values of applied bias voltage the noise is super-Poissonian.

Usually such bistable systems are discussed from the point of view of telegraph noise, which is due to spontaneous random transitions between the two states. This is a consideration complementary to the one we developed above. Indeed, in the linear approximation the system does not know that it is multi-stable. The shot noise grows indefinitely at the instability threshold only because the state around which we have linearized the system becomes unstable rather than metastable. This is a general feature of linear fluctuation theory. Clearly the divergence of shot noise in the linear approximation must be a general feature of all the systems with multistable behavior. Interactions are not the necessary ingredient for this shot noise enhancement. On the other hand, as we have discussed, the transitions between different states, neglected in the linear approximation, will certainly soften the singularity and drive noise to a finite value at the instability threshold. To describe in this way the interplay between shot noise and random telegraph noise remains an open problem.

\section{BOLTZMANN-LANGEVIN APPROACH TO NOISE: DISORDERED SYSTEMS}

\section{A. Fluctuations and the Boltzmann equation}

In this Section we describe the generalization of the Langevin method to disordered systems. As is well known, the evolution of the (average) distribution function $\bar{f}(\boldsymbol{r}, \boldsymbol{p}, t)$ is generally described by the Boltzmann equation,

$$
\left(\partial_{t}+\boldsymbol{v} \nabla+e \boldsymbol{E} \partial_{\boldsymbol{p}}\right) \bar{f}(\boldsymbol{r}, \boldsymbol{p}, t)=I[\bar{f}]+I_{i n}[\bar{f}]
$$

Here $\boldsymbol{E}$ is the local electric field, $I_{i n}[\bar{f}]$ is the inelastic collision integral, due to the electron-electron and electronphonon scattering (we do not have to specify this integral explicitly at this stage), and $I[\bar{f}]$ is the electron-impurity collision integral. For a $d$-dimensional disordered system of volume $\Omega$ it is written as

$$
\begin{aligned}
I[\bar{f}(\boldsymbol{r}, \boldsymbol{p}, t)] & =\Omega \int \frac{d \boldsymbol{p}^{\prime}}{(2 \pi \hbar)^{d}}\left[\bar{J}\left(\boldsymbol{p}^{\prime}, \boldsymbol{p}, \boldsymbol{r}, t\right)-\bar{J}\left(\boldsymbol{p}, \boldsymbol{p}^{\prime}, \boldsymbol{r}, t\right)\right], \\
\bar{J}\left(\boldsymbol{p}, \boldsymbol{p}^{\prime}, \boldsymbol{r}, t\right) & \equiv \tilde{W}\left(\boldsymbol{p}, \boldsymbol{p}^{\prime}, \boldsymbol{r}\right) \bar{f}(\boldsymbol{r}, \boldsymbol{p}, t)\left[1-\bar{f}\left(\boldsymbol{r}, \boldsymbol{p}^{\prime}, t\right)\right],
\end{aligned}
$$

where we have introduced the probability $\tilde{W}$ of scattering per unit time from the state $\boldsymbol{p}$ to the state $\boldsymbol{p}^{\prime}$ due to the impurity potential $U$,

$$
\tilde{W}\left(\boldsymbol{p}, \boldsymbol{p}^{\prime}, \boldsymbol{r}\right)=\frac{2 \pi}{\hbar}\left|U_{\boldsymbol{p} \boldsymbol{p}^{\prime}}\right|^{2} \delta\left[\epsilon(\boldsymbol{p})-\epsilon\left(\boldsymbol{p}^{\prime}\right)\right] .
$$

Thus, the impurity collision integral can be considered as the sum of particle currents $J$ to/from the state $\boldsymbol{p}$ from/to all the possible final states $\boldsymbol{p}^{\prime}$, taken with appropriate signs.

The fluctuations are taken into account via the Boltzmann-Langevin approach, introduced in condensed matter physics by Kogan and Shul'man [244]. This approach assumes that the particle currents between the states $\boldsymbol{p}$ and $\boldsymbol{p}^{\prime}$ fluctuate due to the randomness of the scattering process and partial occupation of the electron states. We write

$$
J\left(\boldsymbol{p}, \boldsymbol{p}^{\prime}, \boldsymbol{r}, t\right)=\bar{J}\left(\boldsymbol{p}, \boldsymbol{p}^{\prime}, \boldsymbol{r}, t\right)+\delta J\left(\boldsymbol{p}, \boldsymbol{p}^{\prime}, \boldsymbol{r}, t\right),
$$

where the average current $\bar{J}$ is given by Eq. (204), and $\delta J$ represents the fluctuations. Then the actual distribution function $f(\boldsymbol{r}, \boldsymbol{p}, t)$, which is the sum of the average distribution $\bar{f}$ and fluctuating part of the distribution $\delta f$, 


$$
f(\boldsymbol{r}, \boldsymbol{p}, t)=\bar{f}(\boldsymbol{r}, \boldsymbol{p}, t)+\delta f(\boldsymbol{r}, \boldsymbol{p}, t),
$$

obeys a Boltzmann equation which contains now in addition a fluctuating Langevin source $\xi$ on the right hand side,

$$
\begin{aligned}
& \left(\partial_{t}+\boldsymbol{v} \nabla+e \boldsymbol{E} \partial_{\boldsymbol{p}}\right) f(\boldsymbol{r}, \boldsymbol{p}, t)=I[f]+I_{i n}[f]+\xi(\boldsymbol{r}, \boldsymbol{p}, t), \\
& \xi(\boldsymbol{r}, \boldsymbol{p}, t)=\Omega \int \frac{d \boldsymbol{p}^{\prime}}{(2 \pi \hbar)^{d}}\left[\delta J\left(\boldsymbol{p}^{\prime}, \boldsymbol{p}, \boldsymbol{r}, t\right)-\delta J\left(\boldsymbol{p}, \boldsymbol{p}^{\prime}, \boldsymbol{r}, t\right)\right] .
\end{aligned}
$$

These Langevin sources are zero on average, $\langle\xi\rangle=0$. To specify the fluctuations, Kogan and Shul'man 244 assumed that the currents $J\left(\boldsymbol{p}, \boldsymbol{p}^{\prime}, \boldsymbol{r}, t\right)$ are independent elementary processes. This means that these currents are correlated only when they describe the same process (identical initial and final states, space point, and time moment); for the same process, the correlations are taken to be those of a Poisson process. Explicitly, we have

$$
\begin{aligned}
& \left\langle\delta J\left(\boldsymbol{p}_{1}, \boldsymbol{p}_{2}, \boldsymbol{r}, t\right) \delta J\left(\boldsymbol{p}_{1}^{\prime}, \boldsymbol{p}_{2}^{\prime}, \boldsymbol{r}^{\prime}, t^{\prime}\right)\right\rangle \\
= & \frac{(2 \pi \hbar)^{2 d}}{\Omega} \delta\left(\boldsymbol{p}_{1}-\boldsymbol{p}_{1}^{\prime}\right) \delta\left(\boldsymbol{p}_{2}-\boldsymbol{p}_{2}^{\prime}\right) \\
\times & \delta\left(\boldsymbol{r}-\boldsymbol{r}^{\prime}\right) \delta\left(t-t^{\prime}\right) \bar{J}\left(\boldsymbol{p}_{1}, \boldsymbol{p}_{2}, \boldsymbol{r}, t\right) .
\end{aligned}
$$

Eq. (208) then implies the following correlations between the Langevin sources,

$$
\left\langle\xi(\boldsymbol{r}, \boldsymbol{p}, t) \xi\left(\boldsymbol{r}^{\prime}, \boldsymbol{p}^{\prime}, t^{\prime}\right)\right\rangle=\delta\left(\boldsymbol{r}-\boldsymbol{r}^{\prime}\right) \delta\left(t-t^{\prime}\right) G\left(\boldsymbol{p}, \boldsymbol{p}^{\prime}, \boldsymbol{r}, t\right),
$$

where

$$
\begin{aligned}
G\left(\boldsymbol{p}, \boldsymbol{p}^{\prime}, \boldsymbol{r}, t\right) & =\Omega\left\{\delta\left(\boldsymbol{p}-\boldsymbol{p}^{\prime}\right) \int d \boldsymbol{p}^{\prime \prime}\right. \\
& \times\left[\bar{J}\left(\boldsymbol{p}^{\prime \prime}, \boldsymbol{p}, \boldsymbol{r}, t\right)+\bar{J}\left(\boldsymbol{p}, \boldsymbol{p}^{\prime \prime}, \boldsymbol{r}, t\right)\right] \\
& \left.-\left[\bar{J}\left(\boldsymbol{p}, \boldsymbol{p}^{\prime}, \boldsymbol{r}, t\right)+\bar{J}\left(\boldsymbol{p}^{\prime}, \boldsymbol{p}, \boldsymbol{r}, t\right)\right]\right\} .
\end{aligned}
$$

Note that the sum rule

$$
\int d \boldsymbol{p} G\left(\boldsymbol{p}, \boldsymbol{p}^{\prime}, \boldsymbol{r}, t\right)=\int d \boldsymbol{p}^{\prime} G\left(\boldsymbol{p}, \boldsymbol{p}^{\prime}, \boldsymbol{r}, t\right)=0,
$$

is fulfilled. This sum rule states that the fluctuations only redistribute the electrons over different states, but do not change the total number of particles.

These equations can be further simplified in the important case (which is the main interest in this Section) when all the quantities are sharply peaked around the Fermi energy. Instead of the momentum $\boldsymbol{p}$, we introduce then the energy $E$ and the direction of the momentum $\boldsymbol{n}=\boldsymbol{p} / p$. The velocity and the density of states are assumed to be constant and equal to $v_{F}$ and $\nu_{F}$, respectively. We write

$$
\Omega \tilde{W}\left(\boldsymbol{p}, \boldsymbol{p}^{\prime}, \boldsymbol{r}\right)=\nu_{F}^{-1} \delta\left(E-E^{\prime}\right) W\left(\boldsymbol{n}, \boldsymbol{n}^{\prime}, \boldsymbol{r}\right),
$$

where $W$ is the probability of scattering from the state $\boldsymbol{n}$ to the state $\boldsymbol{n}^{\prime}$ per unit time at the space point $\boldsymbol{r}$.
Furthermore, we will be interested only in the stationary regime, i.e. the averages $\bar{f}$ and $\bar{J}$ (not the fluctuating parts) are assumed to be time independent. Eliminating the electric field $\boldsymbol{E}$ by the substitution $E \rightarrow E-e \varphi(\boldsymbol{r})$, with $\varphi$ being the potential, we write the BoltzmannLangevin equation in the form

$$
\left(\partial_{t}+v_{F} \boldsymbol{n} \nabla\right) f(\boldsymbol{r}, \boldsymbol{n}, E, t)=I[f]+I_{i n}[f]+\xi(\boldsymbol{r}, \boldsymbol{n}, E, t),
$$

where the Langevin sources $\xi$ are zero on average and are correlated as follows,

$$
\begin{aligned}
& \left\langle\xi(\boldsymbol{r}, \boldsymbol{n}, E, t) \xi\left(\boldsymbol{r}^{\prime}, \boldsymbol{n}^{\prime}, E^{\prime}, t^{\prime}\right)\right\rangle=\frac{1}{\nu_{F}} \delta\left(\boldsymbol{r}-\boldsymbol{r}^{\prime}\right) \delta\left(t-t^{\prime}\right) \\
\times & \delta\left(E-E^{\prime}\right) G\left(\boldsymbol{n}, \boldsymbol{n}^{\prime}, \boldsymbol{r}, E\right), \\
& G\left(\boldsymbol{n}, \boldsymbol{n}^{\prime}, \boldsymbol{r}, E\right) \equiv \int d \boldsymbol{n}^{\prime \prime}\left[\delta\left(\boldsymbol{n}-\boldsymbol{n}^{\prime}\right)-\delta\left(\boldsymbol{n}^{\prime}-\boldsymbol{n}^{\prime \prime}\right)\right] \\
\times & {\left[W\left(\boldsymbol{n}, \boldsymbol{n}^{\prime \prime}, \boldsymbol{r}\right) \bar{f}\left(1-\bar{f}^{\prime \prime}\right)+W\left(\boldsymbol{n}^{\prime \prime}, \boldsymbol{n}, \boldsymbol{r}\right) \bar{f}^{\prime \prime}(1-\bar{f})\right] . }
\end{aligned}
$$

We used the notations $\bar{f} \equiv \bar{f}(\boldsymbol{r}, \boldsymbol{n}, E)$ and $\bar{f}^{\prime \prime} \equiv$ $\bar{f}\left(\boldsymbol{r}, \boldsymbol{n}^{\prime \prime}, E\right)$. The expression for the current density,

$$
\boldsymbol{j}(\boldsymbol{r}, t)=e v_{F} \int d \boldsymbol{n} d E \boldsymbol{n} f(\boldsymbol{r}, \boldsymbol{n}, E, t)
$$

(with the normalization $\int d \boldsymbol{n}=1$ ), completes the system of equations used in the Boltzmann-Langevin method. We remark that in this formulation the local electric potential does not appear explicitly: for systems with charged carriers such as electric conductors the electric field is coupled to the (fluctuating) charge density via the Poisson equation. We will return to this point when we discuss situations in which a treatment of this coupling is essential.

\section{B. Metallic diffusive systems: Classical theory of $1 / 3$ - noise suppression and multi-probe generalization}

Equations (212), (213), and (214) are very general and may be applied to a large variety of systems. Now we turn to the case of metallic diffusive systems, where these equations may be simplified even further and eventually can be solved. A classical theory of noise in metallic diffusive wires was proposed by Nagaev 775, and subsequently by de Jong and Beenakker [89,90]. Sukhorukov and Loss 113, 114 gave another derivation of the shot noise suppression for the two-terminal wire and, more importantly, generalized it to treat conductors of arbitrary geometry and with an arbitrary number of contacts. Below we give a sketch of the derivation, following Ref. [114], to which the reader is addressed for further details.

The distribution function in metallic diffusive systems is almost isotropic. We then separate it into symmetric and asymmetric parts, 


$$
f(\boldsymbol{r}, \boldsymbol{n}, E, t)=f_{0}(\boldsymbol{r}, E, t)+\boldsymbol{n} \boldsymbol{f}_{1}(\boldsymbol{r}, E, t) .
$$

For simplicity, we consider here the relaxation time approximation for the electron-impurity collision integral,

$$
I[f]=-\frac{\boldsymbol{n} \boldsymbol{f}_{1}}{\tau(\boldsymbol{r})}, \frac{n_{\alpha}}{\tau(\boldsymbol{r})}=\int d \boldsymbol{n}^{\prime} W\left(\boldsymbol{n}, \boldsymbol{n}^{\prime}, \mathrm{r}\right)\left[n_{\alpha}-n_{\alpha}^{\prime}\right],
$$

where $\tau$ is the average time a carrier travels between collisions with impurities. This approximation is valid when the scattering is isotropic ( $W$ depends only on the difference $\left.\left|\boldsymbol{n}-\boldsymbol{n}^{\prime}\right|\right)$. The full case is analyzed in Ref. [114. Integrating Eq. (212) first with $\int d \boldsymbol{n}$, and then with $\int \boldsymbol{n} d \boldsymbol{n}$, we obtain

$$
\begin{aligned}
D \nabla \cdot \boldsymbol{f}_{1} & =l \bar{I}_{i n}[f], \quad \bar{I}_{i n}[f] \equiv \int d \boldsymbol{n} I_{i n}[f], \\
\boldsymbol{f}_{1} & =-l \nabla f_{0}+\tau d \int \boldsymbol{n} \xi d \boldsymbol{n},
\end{aligned}
$$

where we have introduced the mean free path $l=v_{F} \tau$ and the diffusion coefficient $D=v_{F} l / d$. We assume the system to be locally charge neutral. Integrating Eq. (217) with respect to energy, we obtain for the local fluctuation of the current

$$
\begin{aligned}
& \delta \boldsymbol{j}+\sigma \nabla \delta \varphi=\delta \boldsymbol{j}^{s}, \quad \nabla \cdot \delta \boldsymbol{j}=0, \\
& \delta \boldsymbol{j}^{s}(\boldsymbol{r}, t)=e l \nu_{F} \int \boldsymbol{n} \xi(\boldsymbol{r}, \boldsymbol{n}, E, t) d \boldsymbol{n} d E .
\end{aligned}
$$

Here $\sigma=e^{2} \nu D$ is the conductivity, and $\varphi(\boldsymbol{r})$ is the electrostatic potential. The currents $\boldsymbol{j}^{s}$ are correlated as follows,

$$
\left\langle\delta j_{l}^{s}(\boldsymbol{r}, t) \delta j_{m}^{s}\left(\boldsymbol{r}^{\prime}, t^{\prime}\right)\right\rangle=2 \sigma \delta_{l m} \delta\left(\boldsymbol{r}-\boldsymbol{r}^{\prime}\right) \delta\left(t-t^{\prime}\right) \Pi(\boldsymbol{r}),
$$

where the quantity $\Pi$ is expressed through the isotropic part of the average distribution function $\bar{f}$,

$$
\Pi(\boldsymbol{r})=\int d E \bar{f}_{0}(\boldsymbol{r}, E)\left[1-\bar{f}_{0}(\boldsymbol{r}, E)\right] .
$$

The distribution $\bar{f}_{0}$ obeys the equation

$$
D \nabla^{2} \bar{f}_{0}(\boldsymbol{r}, E)+\bar{I}_{i n}\left[\bar{f}_{0}-\ln \cdot \nabla \bar{f}_{0}\right]=0 .
$$

The standard boundary conditions for the distribution function are the following. Let $L_{n}$ denote the area of contact $n(1 \leq n \leq N)$, and $\Omega$ the rest of the surface of the sample. At contact $n$, the non-equilibrium distribution function $\bar{f}_{0}$ is determined by the equilibrium Fermi

\footnotetext{
${ }^{54}$ This is the transport mean free path; this definition differs by a numerical factor from that used in Section II. Following the tradition, we are keeping two different definitions for the scattering approach and the kinetic equation approach.
}

distribution function in the reservoir $n,\left.\bar{f}_{0}\right|_{L_{n}}=f_{n}(E)$, whereas away from the contacts the current perpendicular to the surface must vanish and thus $\left.\boldsymbol{N} \cdot \nabla \bar{f}_{0}\right|_{\Omega}=0$, where $\boldsymbol{N}$ is the outward normal to the surface.

Eqs. (218) and (219) can be used to find the currentcurrent fluctuations if the non-equilibrium carrier distribution is known. Thus we proceed first to find the nonequilibrium distribution function, solving Eq. (221). We follow then Ref. [113 and find the characteristic potentials $55 \phi_{n}$, which on the ensemble average obey the Poisson equation $\nabla^{2} \phi_{n}=0$, with the boundary conditions

$$
\left.\phi_{n}\right|_{L_{m}}=\delta_{m n} ;\left.\quad \boldsymbol{N} \cdot \nabla \phi_{n}\right|_{\Omega}=0 .
$$

In terms of the characteristic potentials the electrostatic potential is 136

$$
\varphi(\boldsymbol{r})=\sum_{n} \phi_{n}(\boldsymbol{r}) V_{n}
$$

where $V_{n}$ is the voltage applied to the reservoir $n$. Note that $\sum_{n} \phi_{n}(\boldsymbol{r})=1$ at every space point as a consequence of the invariance of the electrical properties of the conductor under an arbitrary overall voltage shift. With the help of the characteristic potentials, the conductance matrix (which we, as before, define as $I_{m}=G_{m n} V_{n}, I_{m}$ being the current through $L_{m}$ directed into the sample), we obtain

$$
G_{m n}=\sigma \int d \boldsymbol{r} \nabla \phi_{m} \nabla \phi_{n}
$$

(the integration is carried out over the whole sample). The conductances are independent of the electrical (nonequilibrium) potential inside the conductor. To see this one can re-write Eq. (222) in terms of a surface integral.

Multiplying Eq. (218) by $\nabla \phi_{n}$ and integrating over the whole volume we obtain the fluctuation of the current through the contact $n$. The potential fluctuations $\delta \varphi(\boldsymbol{r})$ actually play no role and are eliminated due to the boundary condition that they vanish at the contacts.

\footnotetext{
${ }^{55}$ For an arbitrary conductor the electrostatic potential can be expanded as $\varphi(\boldsymbol{r})=\sum \phi_{n}(\boldsymbol{r}) V_{n}$; Ref. 136 calls the coefficients $\phi_{n}$ characteristic potentials. We remark that in the absence of inelastic processes, the average distribution function can be written as a linear combination of the equilibrium reservoir functions $f_{n}, \bar{f}(\boldsymbol{r})=\nu_{F}^{-1} \sum \nu_{n}(\boldsymbol{r}) f_{n}$. Refs. 110,111. call the coefficients $\nu_{n}$ injectivities. In the diffusive metallic conductor of interest here the characteristic potentials and injectivities are the same functions up to a factor given by the local density of states $\nu_{F}$. Such an equivalence does not hold, for instance, in systems composed of different metallic diffusive conductors, and in general the characteristic potentials and injectivities may have a quite different functional form. Here the use of the characteristic potentials has the advantage that it takes effectively the local charge neutrality into account.
} 
At zero temperature, and to linear order in the applied voltage, this is exact: Internal potential fluctuations play a role only in the non-linear voltage dependence of the shot noise and in its temperature dependence. Taking into account the form of the correlation function (219), we find the noise power,

$$
S_{m n}=4 \sigma \int d \boldsymbol{r} \nabla \phi_{n}(\boldsymbol{r}) \nabla \phi_{m}(\boldsymbol{r}) \Pi(\boldsymbol{r}) .
$$

Eq. (223), together with the equation (221) for the distribution function $\bar{f}_{0}$, is the general result for the multiterminal noise power within the classical approach. At equilibrium $\Pi(\boldsymbol{r})=k_{B} T$, and Eq. (223) reproduces the fluctuation-dissipation theorem.

We next apply Eq. 223) to calculate noise suppression in metallic diffusive wires, for the case when the inelastic processes are negligible, $\bar{I}_{i n}=0$. We consider a wire of length $L$ and width $5 \ll L$, situated along the axis $x$ between the point $x=0$ (reservoir L) and $x=L(\mathrm{R})$. The voltage $V$ is applied to the left reservoir. There are only two characteristic potentials,

$$
\phi_{L}=1-\phi_{R}=1-x / L,
$$

which obey the diffusion equation and do not depend on the transverse coordinate. The average distribution function is found as $f_{0}(x)=\phi_{L}(x) f_{L}+\phi_{R}(x) f_{R}$, and thus the quantity $\Pi$ for zero temperature is expressed as

$$
\Pi(x)=e V \phi_{L}(x)\left[1-\phi_{L}(x)\right] .
$$

Subsequently, we find the conductance $G_{L L}=\sigma W / L$, and the shot noise

$$
S_{L L}=\frac{4 e \sigma W V}{L^{2}} \int_{0}^{L} d x \frac{x}{L}\left(1-\frac{x}{L}\right)=\frac{2 e\langle I\rangle}{3} .
$$

As we mentioned earlier, this expression is due to Nagaev [75]. The Fano factor is $1 / 3$, in accordance with the results found using the scattering approach (Section III).

For purely elastic scattering the distribution function $\bar{f}_{0}$ in an arbitrary geometry quite generally can be written as

$$
\bar{f}_{0}(\boldsymbol{r}, E)=\sum_{n} \phi_{n}(\boldsymbol{r}) f_{n}(E) .
$$

This facilitates the progress for multi-probe geometries. Sukhorukov and Loss 113, 114 obtain general expressions for the multi-terminal noise power and use them to study the Hanbury Brown - Twiss effect in metallic diffusive conductors. The quantum-mechanical theory of the same effect can be found in Ref. [78].

\footnotetext{
${ }^{56}$ We use two-dimensional notations, $d=2$.
}

\section{Interaction effects}

Interaction effects are relatively easy to deal with in the Boltzmann-Langevin approach, in contrast to the difficulties encountered by the scattering theory.

Electron-electron interactions. An important feature of electron-electron interactions is that they do not change the total momentum of the electron system 7 . Generally, therefore, electron-electron scattering alone cannot cause transport, and in particular it cannot cause noise. Technically, this is manifested in the fact that the form of current-current fluctuations is given by the same expression (223), as in the non-interacting case. However, electron-electron scattering processes alter the distribution function $\bar{f}_{0}$, and thus the value of the shot noise.

The inelastic collision integral for electron-electron interactions $\bar{I}_{i n}[f]$ (which we denote $\bar{I}_{e e}[f]$ ) generally has the form

$$
\begin{aligned}
\bar{I}_{e e}[f(E)] & =\int d E^{\prime} d \omega K(\omega)\left\{f(E) f\left(E^{\prime}\right)\right. \\
& \times[1-f(E-\omega)]\left[1-f\left(E^{\prime}+\omega\right)\right] \quad(227) \\
& \left.-f(E-\omega) f\left(E^{\prime}+\omega\right)[1-f(E)]\left[1-f\left(E^{\prime}\right)\right]\right\},
\end{aligned}
$$

where the kernel $K(\omega)$ for disordered systems must be found from a microscopic theory. For three-dimensional metallic diffusive systems it was obtained by Schmid 245]; for two- and one-dimensional systems the zerotemperature result may be found in Ref. [246]. For finite temperatures, a self-consistent treatment is needed 247. This kernel turns out to be a complicated function of disorder and temperature. In particular, in 1D for zero temperature it diverges as $K(\omega) \propto \omega^{-3 / 2}$ for $\omega \rightarrow 0$. The strength of the interaction is characterized by a time $\tau_{e e}$, which we call the electron-electron scattering time.

Thus, one needs now to solve Eq. (221) for $\bar{f}_{0}$, calculate the quantity $\Pi(\boldsymbol{r})$, and substitute it into the expression (223) to obtain the noise. Up to now, only two limiting cases have been discussed analytically. First, for weak interactions $D / L^{2} \gg \tau_{e e}^{-1}$ (with $L$ being the typical size of the system) the collision integral in Eq. (221) may be treated perturbatively. Since electron-electron interactions are of minor importance, the distribution function $\bar{f}_{0}$ in this case is still given by Eq. (226). Nagaev [248], assuming a specific form of $K(\omega)$, found that the shot noise power is enhanced due to the electron-electron interactions compared to the non-interacting result.

Another regime where progress is possible is that of strong scattering, $D / L^{2} \ll \tau_{e e}^{-1}$. In this situation, electrons undergo many scattering events before leaving the

\footnotetext{
${ }^{57}$ This is only correct if Umklapp processes can be neglected. The influence of Umklapp processes on shot noise in mesoscopic systems has not been investigated.
} 
system, and one can expect that the distribution function at every point is close to the equilibrium distribution. Indeed, in the leading order, the diffusion term in Eq. (221) can be neglected, and the distribution function must then be one for which the collision integral is zero. The collision integral (227) vanishes identically for the Fermi distribution, and thus the distribution function assumes the form of a local equilibrium Fermi function with the potential $\varphi(\boldsymbol{r})$ and a local effective temperature $T(\boldsymbol{r})$,

$$
\bar{f}_{0}(\boldsymbol{r}, E)=\left[\exp \left(\frac{E-e \varphi(\boldsymbol{r})}{k_{B} T(\boldsymbol{r})}\right)+1\right]^{-1} .
$$

In the literature this distribution function is also referred to as a hot electron distribution. Eq. 221 is now used to find the effective temperature profile $T(\boldsymbol{r})$. Consider the quantity

$$
w(\boldsymbol{r})=\int d E E\left[\bar{f}_{0}(E)-\theta(E-e \varphi(\boldsymbol{r}))\right],
$$

which up to a coefficient and an additive constant is the total energy of the system. The substitution of Eq. 228) gives $w(\boldsymbol{r})=\pi^{2} T^{2}(\boldsymbol{r}) / 6$. Then the application of Eq. (221) yields

$$
\frac{\pi^{2}}{6} \nabla^{2}\left[T^{2}(\boldsymbol{r})\right]=-\frac{e^{2}}{2} \nabla^{2} \varphi^{2}(\boldsymbol{r})=-e^{2} E^{2}(\boldsymbol{r}),
$$

where we have taken into account $\nabla^{2} \varphi=0$ to obtain the final result. Actually, the term on the right-hand side is proportional to the Joule heating $\boldsymbol{j} \boldsymbol{E}$, and the equation states that this energy losses are spent to heat the electron gas.

In the following, we again specialize to the case of a quasi-one-dimensional metallic diffusive wire between $x=0$ and $x=L$. In this case $E=V / L$, and Eq. 230 must be solved with the boundary conditions $T(0)=T(L)=T_{0}$, with $T_{0}$ being the bath temperature. For the temperature profile we then obtain

$$
T(x)=\left[T_{0}^{2}+\frac{3}{\pi^{2}}\left(\frac{e V}{L}\right)^{2} x(L-x)\right]^{1 / 2} .
$$

Substituting this into Eq. (223), we find the shot noise for hot electrons. In particular, when the bath temperature $T_{0}$ equals zero, we find for the Fano factor

$$
F=\frac{\sqrt{3}}{4} \approx 0.43
$$

The result (231) is due to Kozub and Rudin 249,250 and Nagaev 248]. The multi-terminal generalization is given by Sukhorukov and Loss 113,114]. Eq. (231) states that shot noise for hot electrons is actually higher than for non-interacting electrons. This agrees with the notion which we obtained considering the scattering approach (Section III): Electron heating enhances the shot noise.
Indeed, enhanced (as compared to the 1/3-suppression) shot noise was observed experimentally by Steinbach, Martinis, and Devoret [80]. Their experimental data are shown in Fig. 33 for a particular sample. Shorter samples in the same experiment 80 exhibit Fano factors which are between $1 / 3$ and $\sqrt{3} / 4$. The $1 / 3$-suppression of shot noise and crossover from the diffusive to the hot-electron regime was very carefully studied by Henny et al [81, see subsection II F 4 .

The hot-electron result (232) is actually independent of the details of electron-electron interaction (independent of the kernel $K(\omega)$ in Eq. (227)). The crossover between $F=1 / 3$ and $F=\sqrt{3} / 4$ does depend on this kernel. Nagaev 143 and Naveh [144 studied this crossover numerically for a particular form of $K(\omega)$ which assumes that there is no interference between elastic and electronelectron scattering. They suggested that information on the strength of the electron-electron scattering may be extracted from the zero-frequency noise measurements.

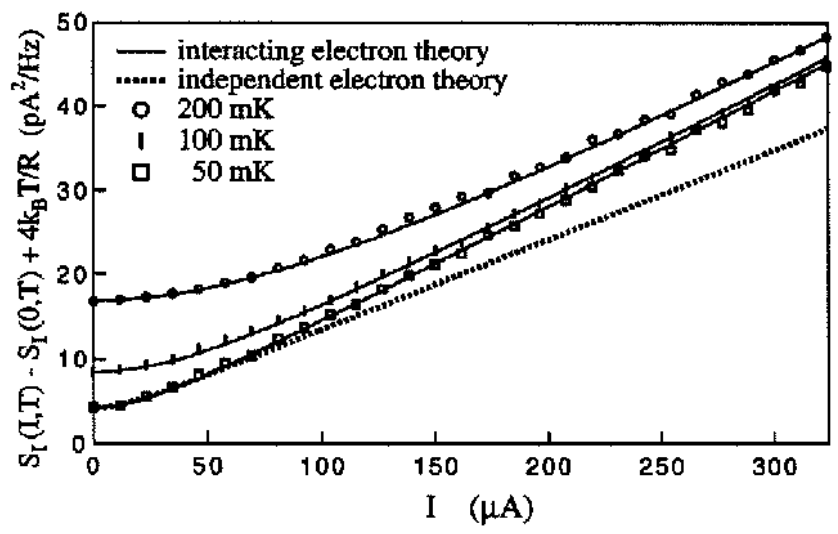

FIG. 33. Shot noise observed at the same sample for three different temperatures by Steinbach, Martinis, and Devoret [30]. Dashed and solid lines indicate the 1/3-suppression and the hot electron result $F=\sqrt{3} / 4$. The temperature is the lowest for the lowest curve.

At this point, we summarize the information we obtained from the scattering and Boltzmann-Langevin approaches about the effects of the electron-electron interactions on noise. There are two characteristic times, one responsible for dephasing processes, $\tau_{\phi}$, and another one due to inelastic scattering (electron heating), $\tau_{e e}$. We expect $\tau_{\phi} \ll \tau_{e e}$. Dephasing does not have an effect on noise, and thus for short enough samples, $D / L^{2} \gg \tau_{e e}^{-1}$, the Fano factor is $1 / 3$ (irrespectively of the relation between $D / L^{2}$ and $\left.\tau_{\phi}\right)$. For long wires, $D / L^{2} \ll \tau_{e e}^{-1}$, the Fano factor equals $\sqrt{3} / 4$ due to electron heating. For even longer wires the electron-phonon interactions become important (see below), and shot noise is suppressed down to zero.

The microscopic theory, however, predicts three characteristic times responsible for electron-electron scattering in disordered systems (for a nice qualitative explanation, see Ref. [251). One is the dephasing time $\tau_{\phi}$, an- 
other one is the energy relaxation time, $\tau_{E}$, and the third one, for which we keep the notation $\tau_{e e}$, has the meaning of the average time between electron-electron collisions. While the dephasing time is quantum-mechanical and cannot be accounted for in the classical theory, the information about $\tau_{e e}$ and $\tau_{E}$ is contained in the collision integral (227). For three-dimensional disordered systems, all the three times coincide: The presence of dephasing always means the presence of inelastic scattering and electron heating. The situation is, however, different in two- and one-dimensional systems, where the three times differ parametrically, with the relation [251,247 $\tau_{e e} \ll \tau_{\phi} \ll \tau_{E}$. This is in apparent contrast with the intuitive predictions of the scattering approach, and opens a number of questions. First, it is not clear whether $\tau_{E}$ or $\tau_{e e}$ is responsible for the $1 / 3-\sqrt{3} / 4$ crossover in the classical theory. Then, the role of dephasing, which is not taken into account in the Boltzmann-Langevin approach, may need to be revisited. These questions may only be answered on the basis of a microscopic theory.

We must point out here that interactions, besides altering the amplitude of shot noise, also create an additional source of noise in diffusive conductors, since the moving electrons produce fluctuating electromagnetic field, as discovered by von Oppen and Stern 252]. Referring the reader to Ref. 252 for details, we mention that this noise is proportional to $V^{2}$ for low voltages, and to $|\omega|^{2 /(4-d)}$ for $D / L^{2} \ll \hbar|\omega| \ll k_{B} T$. As a function of frequency, this noise saturates for low frequencies, and vanishes for $\hbar|\omega| \gg k_{B} T$.

Electron-phonon interactions. In contrast to electron-electron scattering, electron-phonon interactions do change the total momentum of electrons and cause a finite resistance and noise by themselves. Noise in ballistic, one-channel quantum wires due to electron-phonon interactions within the Boltzmann-Langevin approach was studied by Gurevich and Rudin [253,254, who start directly from Eq. (212) with the electron-phonon collision integral on the right-hand side (no impurity scattering). They consider only the situation of weak interactions, when the electron distribution function is not modified by inelastic scattering. They discover that, for this case, the main effect is the absence of noise when the Fermi energy lies below the threshold energy $E_{t h}=2 p_{F} s$, where $s$ is the sound velocity. The appearance of this threshold is due to the fact that the maximal wave vector of acoustic phonons which interact with electrons is $2 p_{F} / \hbar$. For $E_{F}>E_{t h}$ shot noise grows. The suppression of shot noise in long wires, which is a consequence of the equilibration of the electron distribution function due to strong interactions, was beyond the scope of Refs. 253.254.

In disordered systems, one has to take into account three effects. First, elastic scattering modifies the electron-phonon collision integral [255], which assumes different forms depending on the spatial dimension and degree of disorder. Then, it affects the distribution function of electrons. Finally, interactions modify the resistance of the sample, and the expression for the shot noise does not have the simple form of Eq. (223). The distribution function of phonons, which enters the electronphonon collision integral, must be, in principle, found from the Boltzmann equation for phonons, which couples with that for electrons.

The standard approximations used to overcome these difficulties and to get reasonable analytical results are as follows. First, for low temperatures, the contribution to the resistance due to electron-phonon collisions is much smaller than that of electron-impurity scattering. Thus, one assumes that Eq. (223) still holds, and electronphonon collisions only modify the distribution function for electrons and the form of the collision integral. Furthermore, phonons are assumed to be in equilibrium at the lattice temperature. This approach was taken by Nagaev, who calculates the effects of electron-phonon scattering on the shot noise of metallic diffusive wires for the case when electron-electron scattering is negligible [75], and subsequently for the case of hot electrons [248. In addition, careful numerical studies of the role of the electron-phonon interaction in noise in metallic diffusive conductors (starting from the Boltzmann-Langevin approach) are performed by Naveh, Averin, and Likharev [256], and Naveh [145]. The results depend on the relation between temperature, applied voltage, and electronphonon interaction constant, and we refer the reader to Refs. 75,248,256, 145] for details. The only feature we want to mention here is that for constant voltage $e V \gg k_{B} T$ and strong electron-phonon scattering, the Fano factor decreases with the length of the wire. For both non-interacting and hot electrons the specific form of the collision integral which was assumed leads to the dependence $F \propto L^{-2 / 5}$, which is different from the prediction $F \propto L^{-1}$ of the simple model of voltage probes (Section [II). The exponent $2 / 5$ is, however, strongly model dependent, and should not be taken very seriously.

A simple way to see that shot noise is suppressed by the electron-phonon scattering is 249,90 to assume that, in the limit of strong interactions, the distribution function of electrons is the Fermi function with the temperature equal to the bath temperature. Eq. (223) gives then a noise power which is just the Nyquist value, i.e. in this case the shot noise is completely suppressed.

Experimentally, for low temperatures, electron-phonon scattering is less effective than electron-electron collisions, and therefore one expects that, with the increase of the length of the wire, one first goes from the noninteracting regime $F=1 / 3$ to the hot-electron regime, $F=\sqrt{3} / 4$. For even longer wires electron-phonon collisions play a role, and the Fano factor decreases down to zero.

\section{Frequency dependence of shot noise}

While discussing the classical approach to the shot noise suppression, we explicitly assumed that the sample 
is locally charge neutral: Charge pile-up is not allowed in any volume of any size. As a result, we obtained white (frequency independent) shot noise.

In reality, however, there is always a finite (though small) screening radius, which in the case when the system is locally three-dimensional has the form $\lambda_{0}=$ $\left(4 \pi e^{2} \nu\right)^{-1 / 2}$. As Naveh, Averin, and Likharev [140] and Turlakov [257] point out, if one of the dimensions of a disordered sample becomes comparable with $\lambda_{0}$, the pile-up of the charge may modify the frequency dependence of noise, though it leaves the zero-frequency noise power unchanged. If all the dimensions of the sample exceed the screening radius (which is typically the case for metallic, and often also for semiconducting) mesoscopic systems, the charge pile-up inside the sample is negligible, and noise stays frequency independent until at least the plasma frequency, which in three-dimensional structures is very high.

The situation is different if the sample is capacitively connected to an external gate. As we have seen in the framework of the scattering approach (Section IIII), the fact that the sample is now charged, leaves the zerofrequency noise unchanged, but strongly affects the frequency dependence of the shot noise. An advantage of the Boltzmann-Langevin approach is that it can treat these effects analytically, calculating the potential distribution inside the sample and making use of it to treat the current fluctuations. The general program is as follows. Instead of the charge neutrality condition, one uses the full Poisson equation, relating potential and density fluctuations inside the sample. In their turn, density fluctuations are related to the current fluctuations via the continuity equation. Finally, one expresses the current fluctuations via those of the potential and the Langevin sources. Thus, the system of coupled partial differential equations with appropriate boundary conditions needs to be solved. The solution is strongly geometry dependent and has not been written down for an arbitrary geometry. Particular cases, with a simple geometry, were considered by Naveh, Averin, and Likharev [140,142], Nagaev [141,143, and Naveh [144, 145. Without even attempting to give derivations, we describe here the main results, referring the reader to these papers for more details.

A conductor in proximity to a gate can be charged visa-vis the gate. We can view the conductor and the gate as the two plates of a capacitor. In the limit where the screening length is much larger than the wire radius, only a one-dimensional theory is needed. It is this atypical situation which is considered here. For a wire of crosssection $\mathcal{A}$ and a geometrical capacitance $c$ per unit length its low frequency dynamics is characterized by the electrochemical capacitance $c_{\mu}^{-1}=c^{-1}+\left(e^{2} \mathcal{A} \nu\right)^{-1}$ which is

\footnotetext{
${ }^{58}$ For a discussion of electrochemical capacitance and ac conductance see Refs. 157] and 165; see also Section III.
}

the parallel addition of the geometrical capacitance and the quantum capacitance $\left(e^{2} \mathcal{A} \nu\right)^{-1}$. Here we have assumed that the potential is uniform both along the wire and more importantly also in the transverse direction of the wire. Any charge accumulated in the wire can dynamically relax via the reservoirs connected to the wire and via the external circuit which connects the wire and the gate. For a zero-external impedance circuit this relaxation generates a charge relaxation resistance $R_{q}$ (see the discussion in Section III) which for a metallic diffusive conductor is of the order of the sample resistance $R=L(\sigma \mathcal{A})^{-1}$. With these specifications we expect that a metallic diffusive wire in proximity of a gate is characterized by a frequency $\omega_{R C}=1 / R_{q} C_{\mu}$ which is given by $\omega_{R C}=\sigma \mathcal{A} /\left(c L^{2}\right)+\sigma /\left(e^{2} \nu L^{2}\right)$. (Refs. 140,142] express $\omega_{R C}$ in terms of a generalized diffusion constant $D^{\prime}=D+\sigma \mathcal{A} / c$ using the Einstein relation $\sigma=e^{2} \nu D$, such that $\hbar \omega_{R C}=\hbar D^{\prime} / L^{2}$ has the form of a Thouless energy). For $\omega \ll \omega_{R C}$, noise measured at the contacts to the wire is dominated by the white-noise zerofrequency contribution (the Fano factor equals $1 / 3$ for independent electrons or $\sqrt{3} / 4$ for hot electrons). For frequencies higher than $\omega_{R C}$ the spectrum measured at the contacts of the wire starts to depend on the details of the system, and for infinite frequency the Fano factor tends to a constant value, which may lie above as well as below the non-interacting value. This is because the zero-temperature quantum noise $S \propto \hbar|\omega|$ cannot be obtained by classical means: Thus, all the results of this subsections are applicable only outside of the regime when this source of noise is important. In particular, for zero temperature this means $\omega<e|V|$. The crossover to the quantum noise was recently treated by Nagaev 214. using the Green's functions technique.

It is also assumed that the frequency is much below the inverse elastic scattering time, $\omega \ll \tau^{-1}$; outside this regime, the diffusion approximation is not valid. As emphasized in work on chaotic cavities 98 experiments which measure the noise at the gate can also be envisioned: This has the advantage that even for frequencies much smaller than $\omega_{R C}$ the noise is frequency dependent and in fact can for metallic systems also be expected to be determined by $R_{q}$ and $C_{\mu}$.

Nagaev [141,143] considers a circular conductor of length $L$ and radius $R$, surrounded by a circular gate. For $L \ll R$ (short wire) he finds that the noise spectrum measured at a contact is frequency independent. However, generally the frequency dependence is quite pronounced. Thus, for the case when the distribution function is described by Eq. (226), and wires are long, $L \gg R$, the correlation of currents taken at the same contact, $S_{L L}$, for $\omega \gg \omega_{c}$ tends to the Poisson value $2 e\langle I\rangle$, while the current correlation at different contacts, $S_{L R}$, rapidly falls off with frequency, and is exponentially small for $\omega \gg \omega_{c}$. Note that the current is not conserved in this system, since an ac current is also generated at the gate. For hot electrons, the high-frequency noise is 
given by [143] $S_{L L} \sim e\langle I\rangle \alpha^{1 / 6}$, where $\alpha$ is a dimensionless parameter proportional to the interaction strength. This result seems paradoxical, since for the hot electrons the distribution function is independent of the form of the collision integral, and thus the results for noise at any frequency are not expected to depend on this form. The resolution of this paradox, as pointed out by Nagaev 1143, is that close to the contacts for hot electrons the term $\nabla^{2} f$ diverges, and in some sense the electrons close to the contacts are never hot.

Naveh, Averin, and Likharev 142 consider analytically a geometry in which a planar contact is situated above a gate. Instead of solving the Poisson equation, they assumed that the potential profile $\varphi(x)$ is proportional to the charge density $\rho(x)$ in the insulating layer which separates the sample from the gate. Thus, the screening radius is set to be zero, and the charge pileup in the sample is effectively forbidden 5 . They studied the current correlation at the same cross-section, $S(x)$. For non-thermalized electrons, the high-frequency noise $\left(\omega \gg \omega_{c}\right)$ inside the conductor grows even for zero temperature, $S(x) \propto\left(\omega / \omega_{c}\right)^{1 / 2}[x(L-x)]^{1 / 2}$. At the contacts this noise power turns to zero, and one obtains $S_{L L} \equiv S(x=0)=e\langle I\rangle$. Note that this value is the same as for a double-barrier structure with symmetric capacitances. The difference with the result $S_{L L}=2 e\langle I\rangle$ originates from the fact that in this mode the charge pileup in the contact is forbidden. In addition, the frequency dependent noise in the same model is studied numerically by Naveh for electron-electron [144 and electron-phonon 145] interactions.

\section{E. Shot noise in non-degenerate conductors}

Diffusive conductors. Recently, González et al [258], motivated by the diversity of proofs for the $1 / 3$ noise suppression in metallic diffusive wires, performed numerical simulations of shot noise in non-degenerate diffusive conductors. They have taken the interaction effects into account via the Poisson equation. For the disorder a simple model with an energy independent scattering time was assumed. They found that the noise suppression factor for this system, within the error bars, equals $1 / 3$ and $1 / 2$ in three- and two-dimensional systems, respectively. A subsequent work 259] gives the suppression factor 0.7 for $d=1$.

An analytic theory of shot noise in these conductors was proposed by Beenakker [260], and subsequently by Nagaev 261 and Schomerus, Mishchenko, and Beenakker [262,263. The general conclusion is as

\footnotetext{
${ }^{59}$ Numerical results for the same model with charge pile-up (only non-thermalized electrons) were previously provided by Nagaev 141].
}

follows. Shot noise in non-degenerate diffusive conductors is non-universal in the sense that it depends on the details of the disorder (the energy dependence of the elastic scattering time) and the geometry of the sample. In the particular case, when the elastic relaxation time is energy independent (corresponding to the simulations by González et al [258,259]), the suppression factors are close to $1 / 3,1 / 2$, and 0.7 , but not precisely equal to these values. Below we give a brief sketch of the derivation, following Ref. 262].

We start from the Boltzmann equation (203) and add Langevin sources on the right-hand side. Three points now require special attention: (i) for a degenerate gas $\bar{f} \ll 1$, and thus the factors $(1-\bar{f})$ in the collision integral (204) can be taken equal to 1 ; (ii) since carriers are now distributed over a wide energy range, all the quantities must be taken energy-dependent rather than restricted to the Fermi surface. In particular, the velocity is $v(E)=(2 E / m)^{1 / 2}$, and the energy dependence of the mean free path matters, (iii) the screening length is energy dependent, and the system generally may not be regarded as charge neutral. We employ again the $\tau$-approximation for the electron-impurity collision integral. For a while, we assume the relaxation time $\tau$ to be energy independent. We also neglect all kinds of inelastic scattering. Separating the distribution function into symmetric and asymmetric parts (216), and introducing the (energy resolved) charge and current densities,

$$
\begin{array}{r}
\rho(\boldsymbol{r}, E, t)=e \nu(E) f_{0}(\boldsymbol{r}, E, t) ; \\
\boldsymbol{j}(\boldsymbol{r}, E, t)=\frac{1}{d} e v(E) \nu(E) \boldsymbol{f}_{1}(\boldsymbol{r}, E, t),
\end{array}
$$

we obtain two equations, analogous to Eq. (217),

$$
\nabla \cdot \boldsymbol{j}+e \boldsymbol{E}(\boldsymbol{r}, t) \partial_{E} \boldsymbol{j}=0
$$

and

$$
\boldsymbol{j}=-D(E) \nabla \rho-\sigma(E) \boldsymbol{E}(\boldsymbol{r}, t) \partial_{E} f_{0}+\delta \boldsymbol{j}(\boldsymbol{r}, E, t)=0 .
$$

Here $D(E)=2 E \tau / m d$ and $\sigma(E)=e^{2} \nu(E) D(E)$ are the energy dependent diffusion coefficient and conductivity, respectively. The electric field $\boldsymbol{E}$ is coupled to the charge density via the Poisson equation,

$$
\nabla \cdot \boldsymbol{E}(\boldsymbol{r}, t)=\int d E \rho(\boldsymbol{r}, E, t),
$$

and the energy resolved Langevin currents $\delta \boldsymbol{j}$ are correlated in the following way (cf. Eq. (219)),

$$
\begin{aligned}
& \left\langle\delta j_{l}(\boldsymbol{r}, E, t) \delta j_{m}\left(\boldsymbol{r}^{\prime}, E^{\prime}, t^{\prime}\right)\right\rangle=2 \sigma(E) \delta_{l m} \delta\left(\boldsymbol{r}-\boldsymbol{r}^{\prime}\right) \\
\times & \delta\left(E-E^{\prime}\right) \delta\left(t-t^{\prime}\right) \bar{f}_{0}(\boldsymbol{r}, E, t) .
\end{aligned}
$$

Equations (233), (234), and 235) describe the response of the system to the fluctuations (236) of the Langevin currents. The main complication as compared 
to the degenerate case is that the fluctuations of the electric field now play an essential role, and cannot be neglected. For this reason, results for the shot noise depend dramatically on the geometry.

Further progress can be achieved for the quasi-onedimensional geometry of a slab $0<x<L$, located between two reservoirs of the same cross-section. One more approximation is the regime of space-charge limiting conduction, corresponding to the boundary condition $\boldsymbol{E}(x=0, t)=0$. This condition means that the charge in the contacts is well screened, $L \gg L_{s} \gg L_{c}$, with $L_{c}$ and $L_{s}$ being the screening length in the contacts and the sample, respectively. Equations (233), (234), and (235) are supplemented by the absorbing boundary condition at another contact, $\rho(x=L, t)=0$.

Now one can calculate the potential profile inside the sample and subsequently the shot noise power. In this way, within the approximation of energy independent relaxation times, Schomerus, Mishchenko, and Beenakker [262] obtain the following results,

$$
F= \begin{cases}0.69, & d=1 \\ 0.44, & d=2 \\ 0.31, & d=3\end{cases}
$$

The numerical values (237) are, indeed, close to $0.7,1 / 2$ and $1 / 3$, respectively, in accordance with the numerical results by González et al 258,259], but not precisely equal to them.

It is now worthwhile to mention that the results (237), in contrast to the $1 / 3$-suppression of shot noise in the degenerate diffusive conductors, are not universal. Whereas the $1 / 3$ suppression is independent of the geometry of the sample, degree of the disorder, or local dimensionality, the values (237), being geometry independent, do depend on the dimensionality of the sample. Furthermore, they $d o$ depend on the disorder, and this dependence enters through their sensitivity to the energy dependence of the relaxation time 0 , as noticed by Nagaev 261. Schomerus, Mishchenko, and Beenakker [263] investigated the case $\tau(E) \propto E^{\alpha},-1 / 2 \leq \alpha \leq 1$, which are the only values of $\alpha$ compatible with the regime of space-charge limited conduction. In particular, $\alpha=-1 / 2$ corresponds to scattering on short-ranged impurities. They found that the Fano factor in $d=3$ crosses over monotonically from $F=0.38(\alpha=-1 / 2)$ to $F=0(\alpha=1)$. There is no shot noise in this model for $\alpha>1$.

This example clearly demonstrates that Fermi statistics are not necessary to suppress shot noise, in accordance with general expectations.

Ballistic non-degenerate conductors. Bulashenko, Rubí, and Kochelap 264] address the noise in

\footnotetext{
${ }^{60}$ In the case of metallic diffusive wires, this dependence is irrelevant since the relaxation time is evaluated at the Fermi surface.
}

charge limited ballistic conductors. They consider a twoterminal semiconductor sample with heavily doped contacts. Carriers in the semiconductor exist only due to injection from the contacts which thus determine the potential distribution inside the sample. The self-consistent field determines a barrier at which carriers are either completely reflected or completely transmitted (no tunneling). This system is thus a close analog of the charge limited shot noise in vacuum tubes.

It is easy to adapt the Boltzmann-Langevin formulation to this problem: Since carrier motion inside the conductor is determined by the Vlasov equation (collisionless Boltzmann equation),

$$
\left(\partial_{t}+v_{x} \partial_{x}+e E_{x} \partial_{p_{x}}\right) f(x, \boldsymbol{p}, t)=0,
$$

the distribution function $f(x, \boldsymbol{p}, t)$ at any point inside the sample is determined by the distribution function at the surface of the sample. The only source of noise arises from the random injection of carriers at the contacts. Thus the boundary conditions are

$$
\begin{aligned}
\left.f(0, \boldsymbol{p}, t)\right|_{v_{x}>0} & =f_{L}+\delta f_{L}(\boldsymbol{p}, t), \\
\left.f(L, \boldsymbol{p}, t)\right|_{v_{x}<0} & =f_{R}+\delta f_{R}(\boldsymbol{p}, t) .
\end{aligned}
$$

The stochastic forces $\delta f_{L, R}$ are zero on average, and their correlation is

$$
\left\langle\delta f_{\alpha}(\boldsymbol{p}, t) \delta f_{\beta}\left(\boldsymbol{p}^{\prime}, t^{\prime}\right)\right\rangle \propto \delta_{\alpha \beta} \delta\left(p_{x}-p_{x}^{\prime}\right) \delta\left(t-t^{\prime}\right) f_{\alpha}\left(p_{x}\right),
$$

where $f_{\alpha}\left(p_{x}\right)$ is the Maxwell distribution function restricted to $p_{x}>0(\alpha=L)$ or $p_{x}<0(\alpha=R)$. Note that in the degenerate case we would have to write an extra factor $1-f_{\alpha}$ on the right-hand side of Eq. (240), thus ensuring that there is no noise at zero temperature. We have checked already in Section [1] that this statement is correct. On the contrary, in the non-degenerate case $f_{\alpha} \ll 1$, and thus $\left(1-f_{\alpha}\right) \sim 1$. The results for this regime do not of course allow an extrapolation to $k_{B} T=0$. The crossover between the shot noise behavior in degenerate and non-degenerate conductors was investigated by Gonzàlez et al [265] using Monte Carlo simulations.

Eq. (238) is coupled to the Poisson equation,

$$
-d E_{x} / d x=4 \pi \sum_{\boldsymbol{p}} f(x, \boldsymbol{p}, t) .
$$

Solving the resulting equations, Bulashenko, Rubí, and Kochelap 264 found that interactions, at least in a certain parameter range, suppress shot noise below the Poisson value. The suppression may be arbitrarily strong in long (but still ballistic) samples. The results are in good agreement with previous numerical studies of shot noise in the same system by González et al [266,268] and Bulashenko et al [267].

The crossover between the ballistic and diffusive behavior of the non-degenerate Fermi gas was numerically 
studied by González et al [268,259. Analytical results on this crossover are presently unavailable.

A similar problem, a ballistic degenerate conductor in the presence of a nearby gate, was posed by Naveh, Korotkov, and Likharev 269.

We conclude this subsection by recalling that the effect of interactions on noise arises not only in long ballistic structures but already in samples which are effectively zero dimensional, like resonant tunneling diodes 78 or in quantum point contacts [98]. The added complication in extended structures arises from the long range nature of the Coulomb interaction.

\section{F. Boltzmann-Langevin method for shot noise suppression in chaotic cavities with diffusive boundary scattering}

Now we turn to the classical derivation of the $1 / 4-$ shot noise suppression in chaotic cavities. In standard cavities, which are regular objects, the chaotic dynamics arises due to the complicated shape of a surface. Thus scattering at the surface is deterministic and in an individual ensemble member scattering along the surface of the cavity is noiseless. Thus it is not obvious how to apply the Boltzmann-Langevin equation.

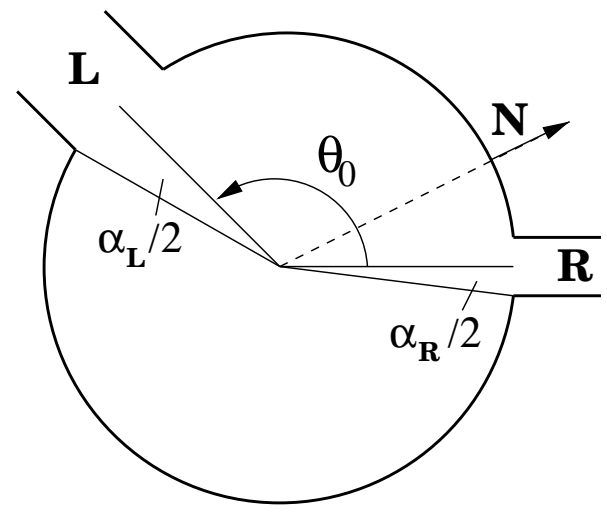

FIG. 34. Geometry of the chaotic cavity with diffusive boundary scattering.

However, recently a model of a random billiard - a circular billiard with diffusive boundary scattering — was proposed 270,271 to emulate the behavior of chaotic cavities 67 . It turned out that the model can be relatively easily dealt with, and Refs. 270,271 used it to study spectral and eigenfunction properties of closed systems. Ref. 99] suggests that the same model may be used to study the transport properties of the open chaotic cavities and presents the theory of shot noise based on the

\footnotetext{
${ }^{61}$ Earlier, a similar model was numerically implemented to study spectral statistics in closed square billiards 272,273.
}

Boltzmann-Langevin approach.

We consider a circular cavity of radius $R$ connected to the two reservoirs via ideal leads; the angular positions of the leads are $\theta_{0}-\alpha_{L} / 2<\theta<\theta_{0}+\alpha_{L} / 2$ (left) and $-\alpha_{R} / 2<\theta<\alpha_{R} / 2$ (right), see Fig. 34; $\theta$ is the polar angle. The contacts are assumed to be narrow, $\alpha_{L, R} \ll 1$, though the numbers of the transverse channels, $N_{L, R}=$ $p_{F} R \alpha_{L, R} / \pi \hbar$, are still assumed to be large compared to 1. Inside the cavity, motion is ballistic, and the average distribution function $\bar{f}(\boldsymbol{r}, \boldsymbol{n})$ obeys the equation

$$
\boldsymbol{n} \nabla \bar{f}(\boldsymbol{r}, \boldsymbol{n})=0 .
$$

At the surface (denoted by $\Omega$ ) we can choose a diffusive boundary condition: the distribution function of the particles backscattered from the surface is constant (independent of $\boldsymbol{n}$ ) and fixed by the condition of current conservation $^{62}$,

$$
\bar{f}(\boldsymbol{r}, \boldsymbol{n})=\pi \int_{\left(\boldsymbol{N} \boldsymbol{n}^{\prime}>0\right)}\left(\boldsymbol{N} \boldsymbol{n}^{\prime}\right) \bar{f}\left(\boldsymbol{r}, \boldsymbol{n}^{\prime}\right) d \boldsymbol{n}^{\prime}, \quad \boldsymbol{N} \boldsymbol{n}^{\prime}<0
$$

where $\boldsymbol{r} \in \Omega, \boldsymbol{N}$ is the outward normal to the surface, and $\int d \boldsymbol{n}=1$. Furthermore, we assume that the electrons coming from the leads are described by the equilibrium distribution functions, and are emitted uniformly into all directions. Explicitly, denoting the cross-sections of the left and the right leads by $\Omega_{L}$ and $\Omega_{R}$, we have

$$
\bar{f}(\boldsymbol{r}, \boldsymbol{n})=f_{L, R}, \quad \boldsymbol{r} \in \Omega_{L, R} ; \quad \boldsymbol{N} \boldsymbol{n}<0 .
$$

Now we can find the average distribution function. Since motion away from the boundary is ballistic, the value of the distribution function Eq. (242) at a point away from the boundary, is determined by the distribution function at the surface associated with the trajectory that reaches this point after a scattering event at the surface. With the boundary conditions (243) and (244), we can then derive an integral equation for $\bar{f}(\theta)$,

$$
\left.\bar{f}(\theta)\right|_{\Omega}=\frac{1}{4} \int_{\Omega+\Omega_{L}+\Omega_{R}} \bar{f}\left(\theta^{\prime}\right)\left|\sin \frac{\theta-\theta^{\prime}}{2}\right| d \theta^{\prime},
$$

subject to the additional conditions $\left.\bar{f}(\theta)\right|_{\Omega_{L, R}}=f_{L, R}$. This exact equation may be considerably simplified in the limit of narrow leads, $\alpha_{L, R} \ll 1$. Integrals of the type $\int_{\Omega_{R}} F(\theta) d \theta$ can now be replaced by $\alpha_{R} F(0)$. This gives for the distribution function

$$
\bar{f}(\theta)=\frac{\alpha_{L} f_{L}+\alpha_{R} f_{R}}{\alpha_{L}+\alpha_{R}}
$$

\footnotetext{
${ }^{62}$ This is the simplest possible boundary condition of this kind. For a review, see Ref. 274. We expect similar results for any other diffusive boundary condition.
} 


$$
\begin{aligned}
& +\frac{g(0)-g\left(\theta_{0}\right)}{4 \pi} \frac{\alpha_{L} \alpha_{R}\left(\alpha_{L}-\alpha_{R}\right)}{\left(\alpha_{L}+\alpha_{R}\right)^{2}}\left(f_{L}-f_{R}\right) \\
& +\frac{g(\theta)-g\left(\theta-\theta_{0}\right)}{4 \pi} \frac{\alpha_{L} \alpha_{R}}{\left(\alpha_{L}+\alpha_{R}\right)}\left(f_{L}-f_{R}\right),
\end{aligned}
$$

with the notation

$$
g(\theta)=\sum_{l=1}^{\infty} \frac{\cos l \theta}{l^{2}}=\frac{1}{12}\left(3 \theta^{2}-6 \pi \theta+2 \pi^{2}\right), \quad 0 \leq \theta \leq 2 \pi .
$$

The first part of the distribution function (246) does not depend on energy and corresponds to the random matrix theory (RMT) results for the transport properties. The second two terms on the right hand side are not universal and generate sample-specific corrections to RMT [99], which we do not discuss here.

The conductance is easily found to be

$$
G=\frac{e^{2}}{2 \pi \hbar} \frac{N_{L} N_{R}}{N_{L}+N_{R}}
$$

which is identical to the RMT result.

The main problem we encounter in attempting to calculate noise via the Boltzmann-Langevin method is that the system is not described by a collision integral of the type (204). Instead, the impurity scattering is hidden inside the boundary condition (243), which, in principle, itself must be derived from the collision integral. This difficulty can, however, be avoided, since we can calculate the probability $W\left(\boldsymbol{n}, \boldsymbol{n}^{\prime}, \boldsymbol{r}\right)$ of scattering per unit time from the state $\boldsymbol{n}$ to the state $\boldsymbol{n}^{\prime}$ at the point $\boldsymbol{r}$ (which is, of course, expected to be non-zero only at the diffusive boundary). Indeed, this probability is

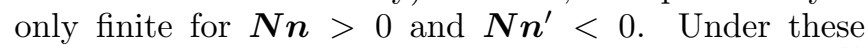
conditions it does not depend on $\boldsymbol{n}^{\prime}$, and thus equals ${ }^{63}$ $W\left(\boldsymbol{n}, \boldsymbol{n}^{\prime}, \boldsymbol{r}\right)=2 W^{\prime}(\boldsymbol{n}, \boldsymbol{r})$, with $W^{\prime}$ being the probability per unit time to scatter out of the state $\boldsymbol{n}$ at the space point $\boldsymbol{r}$. Imagine now the (short) time interval $\Delta t$. During this time, the particles which are closer to the surface than $v_{F} \boldsymbol{n} \boldsymbol{N} \Delta t$ are scattered with probability one, and others are not scattered at all. Taking the limit $\Delta t \rightarrow 0$, we obtain

$$
W\left(\boldsymbol{n}, \boldsymbol{n}^{\prime}, \boldsymbol{r}\right)=\left\{\begin{array}{cc}
v_{F} \boldsymbol{n} \boldsymbol{N} \delta(R-r), & \boldsymbol{n} \boldsymbol{N}>0, \boldsymbol{n}^{\prime} \boldsymbol{N}<0 \\
0, & \text { otherwise }
\end{array} .\right.
$$

Imagine now that we have a collision integral, which is characterized by the scattering probabilities (247). Then, the fluctuation part of the distribution function $\delta f$ obeys the equation 54 (212) with the Langevin sources correlated according to Eq. (213). A convenient way to proceed was proposed by de Jong and Beenakker [89, 90],

\footnotetext{
${ }^{63}$ The coefficient 2, instead of $\pi^{-1}$, is due to the normalization.

${ }^{64}$ We assume that there is no inelastic scattering, $I_{\text {in }} \equiv 0$.
}

who showed that quite generally the expression for the noise can be brought into the form

$$
\begin{aligned}
S & =2 e^{2} \nu_{F} \int d E \int d \boldsymbol{n} d \boldsymbol{n}^{\prime} d \boldsymbol{r} T_{R}(\boldsymbol{n}, \boldsymbol{r}) T_{R}\left(\boldsymbol{n}^{\prime}, \boldsymbol{r}\right) \\
& \times G\left(\boldsymbol{n}, \boldsymbol{n}^{\prime}, \boldsymbol{r}, E\right),
\end{aligned}
$$

where the function $G$ is given by Eq. (214), and $T_{R}$ is the probability that the particle at $(\boldsymbol{n}, \boldsymbol{r})$ will eventually exit through the right lead. This probability obeys $n \nabla T_{R}=0$ with the diffusive boundary conditions at the surface; furthermore, it equals 0 and 1 provided the particle is headed to $\Omega_{L}$ and $\Omega_{R}$, respectively. In the leading order $T_{R}=\alpha_{R} /\left(\alpha_{L}+\alpha_{R}\right)$; however, this order does not contribute to the noise due to the sum rule (211). The subleading order is that for $\boldsymbol{n}$ pointing out of the left (right) contact, $T_{R}=0(1)$. Substituting the distribution function $\bar{f}=\left(\alpha_{L} f_{L}+\alpha_{R} f_{R}\right) /\left(\alpha_{L}+\alpha_{R}\right)$ into Eq. 248), we obtain for the Fano factor

$$
F=\frac{N_{L} N_{R}}{\left(N_{L}+N_{R}\right)^{2}} .
$$

We have thus presented a purely classical derivation of the Fano factor of a chaotic cavity (96). This result was previously derived with the help of the scattering approach and RMT theory (subsection IIF 5).

To what extent can the billiard with diffusive boundary scattering also describe cavities which exhibit deterministic surface scattering? As we have stated above, if we consider an ensemble member of a cavity with specular scattering at the surface, such scattering is deterministic and noiseless. Thus we can definitely not expect the model with a diffusive boundary layer to describe an ensemble member. However, to the extent that we are interested in the description only of ensemble averaged quantities (which we are if we invoke a Boltzmann-Langevin equation) the diffusive boundary layer model can also describe the ensemble averaged behavior of cavities which are purely deterministic. While in an individual cavity, a particle with an incident direction and velocity generates a definite reflected trajectory, we can, if we consider the ensemble average, associate with each incident trajectory, a reflected trajectory of arbitrary direction. In the ensemble, scattering can be considered probabilistic, and the diffusive boundary model can thus also be used to describe cavities with completely deterministic scattering at the surface. This argument is correct, if we can commute ensemble and statistical averages. To investigate this further, we present below another discussion of the deterministic cavity.

\section{G. Minimal correlation approach to shot noise in deterministic chaotic cavities}

In cavities of sufficiently complicated shape, deterministic chaos appears due to specular scattering at the surface. To provide a classical description of shot noise in 
this type of structures, Ref. 99] designed an approach which it called a "minimal correlation" approach. It is not clear whether it can be applied to a broad class of systems, and therefore, we decided to put it in this Section rather than to provide a separate Section.

In a mesoscopic conductor, in the presence of elastic scattering only, the distribution function is quite generally given by 110,111

$$
f_{0}(\boldsymbol{r})=\sum_{n} \frac{\nu_{n}(\boldsymbol{r})}{\nu(\boldsymbol{r})} f_{n}
$$

where $\nu_{n}(\boldsymbol{r})$ is the injectivity of contact $n$ (the contribution to the local density of states $\nu(\boldsymbol{r})$ of contact $n$.) For the ensemble averaged distribution which we seek, we can replace the actual injectivities and the actual local density of states by their ensemble average. For a cavity with classical contacts $\left(N_{n} \gg 1\right.$ open quantum channels), the ensemble averaged injectivities are 138 $\left\langle\nu_{n}(\boldsymbol{r})\right\rangle=\nu_{F} N_{n} / \sum_{n} N_{n}$, where $\nu_{F}$ is the ensemble averaged local density of states. This just states that the contribution of the $n$-th contact to the local density of states is proportional to its width number of quantum channels). Thus the ensemble averaged distribution function, which we denote by $f_{C}$, is

$$
f_{C}=\sum_{n} \beta_{n} f_{n}, \quad \beta_{n} \equiv N_{n} / \sum_{n} N_{n} .
$$

To derive this distribution function classically, we first assert that the ensemble averaged distribution inside the cavity, called $f_{C}$, is a spatially independent constant. This is a consequence of the fact that after ensemble averaging at any given point within the cavity there are no preferred directions within the cavity if carriers conserve their energy (no inelastic scattering). On the ensemble average the interior of the cavity can be treated as an additional dephasing voltage probe (see subsection II G). The interior acts as a dephasing probe since we assume that there is no inelastic scattering at the probe. Consequently at such a probe the current in each energy interval is conserved [90]. Thus we must consider the energy resolved current.

Let us denote the current at contact $n$ in an energy interval $d E$ by $J_{n}(E)$. The total current at the contact is $I_{n}=\int J_{n} d E$. In terms of the distribution function of the reservoirs and the cavity the energy-resolved current is

$$
J_{n}(E)=e^{-1} G_{n}\left(f_{n}-f_{C}\right),
$$

where $G_{n}=e^{2} N_{n} /(2 \pi \hbar)$ is the (Sharvin) conductance of the $n$th contact, and $N_{n}=p_{F} W_{n} / \pi \hbar$ is the number of transverse channels. For energy conserving carrier motion the sum of all currents in each energy interval must vanish (subsection II G). This requirement immediately gives Eq. (250).

Using the distribution function Eq. (250) gives for the conductance matrix

$$
G_{m n}=\left(\delta_{m n}-\beta_{m}\right) G_{n}
$$

This conductance matrix is symmetric, and for the twoterminal case becomes $G_{L L}=\left(e^{2} / 2 \pi \hbar\right)\left(N_{L} N_{R} /\left(N_{L}+\right.\right.$ $\left.N_{R}\right)$ ), as expected.

Now we turn to the shot noise. The fluctuation of the current through the contact $n$ is written as

$$
\delta I_{n}=-\frac{e p_{F}}{2 \pi \hbar^{2}} \int_{\Omega_{n}} d \boldsymbol{n} d \boldsymbol{r} d E\left(\boldsymbol{n} \boldsymbol{N}_{n}\right) \delta f(\boldsymbol{r}, \boldsymbol{n}, E, t),
$$

where $\Omega_{n}$ and $\boldsymbol{N}_{n}$ denote the surface of the contact $n$ and the outward normal to this contact. Here $\delta f$ is the fluctuating part of the distribution function, and for further progress we must specify how these fluctuations are correlated.

The terms with $\boldsymbol{n} \boldsymbol{N}_{n}<0$ describe fluctuations of the distribution functions of the equilibrium reservoirs, $f_{n}(E)$. These functions fluctuate due to partial occupation of states (equilibrium noise); the fluctuations of course vanish for $k_{B} T=0$. The equal time correlator of these equilibrium fluctuations quite generally is (see e.g. Ref. 275)

$$
\begin{aligned}
& \left\langle\delta f(\boldsymbol{r}, \boldsymbol{n}, E, t) \delta f\left(\boldsymbol{r}^{\prime}, \boldsymbol{n}^{\prime}, E^{\prime}, t\right)\right\rangle \\
= & \nu_{F}^{-1} \delta\left(\boldsymbol{r}-\boldsymbol{r}^{\prime}\right) \delta\left(\boldsymbol{n}-\boldsymbol{n}^{\prime}\right) \delta\left(E-E^{\prime}\right) \\
\times & \bar{f}(\boldsymbol{r}, \boldsymbol{n}, E, t)[1-\bar{f}(\boldsymbol{r}, \boldsymbol{n}, E, t)],
\end{aligned}
$$

where in the reservoirs $\bar{f}=f_{n}(E)$. In particular, the cross correlations are completely suppressed.

On the other hand, the terms with $\boldsymbol{n} \boldsymbol{N}_{n}>0$ describe fluctuations of the distribution function inside the cavity. These non-equilibrium fluctuations resemble Eq. (254) very much. Indeed, in the absence of random scattering the only source of noise are the fluctuations of the occupation numbers. Furthermore, in the chaotic cavity the cross correlations should be suppressed because of multiple random scattering inside the cavity. Thus, we assume that Eq. (254) is valid for fluctuations of the non-equilibrium state of the cavity, where the function $f_{C}(E)$ 250) plays the role of $\bar{f}(\boldsymbol{r}, \boldsymbol{n}, E, t)$. In contrast to the true equilibrium state, these fluctuations persist even for zero temperature, since the average distribution function (250) differs from both zero and one.

Furthermore, for $t \neq t^{\prime}$ the correlator obeys the kinetic equation, $\left(\partial_{t}+v_{F} \boldsymbol{n} \nabla\right)\left\langle\delta f(t) \delta f\left(t^{\prime}\right)\right\rangle=0$ [275. We obtain the following formula,

$$
\begin{aligned}
& \left\langle\delta f(\boldsymbol{r}, \boldsymbol{n}, E, t) \delta f\left(\boldsymbol{r}^{\prime}, \boldsymbol{n}^{\prime}, E^{\prime}, t^{\prime}\right)\right\rangle \\
& =\nu_{F}^{-1} \delta\left[\boldsymbol{r}-\boldsymbol{r}^{\prime}-v_{F} \boldsymbol{n}\left(t-t^{\prime}\right)\right] \\
& \times \delta\left(\boldsymbol{n}-\boldsymbol{n}^{\prime}\right) \delta\left(E-E^{\prime}\right) f_{C}\left(1-f_{C}\right),
\end{aligned}
$$

which describes strictly ballistic motion and is therefore only valid at the time scales below the time of flight. An attempt to use Eq. (255) for all times and insert it to Eq. (253) immediately leads to the violation of current conservation. 
Thus, we must take special care of the fluctuations of the distribution function inside the cavity for the case when $t-t^{\prime}$ is much longer than the dwell time $\tau_{d}$. In this situation, the electron becomes uniformly distributed and leaves the cavity through the $n$th contact with the probability $\beta_{n}$. For times $t \gg \tau_{d}$ (which are of interest here) this can be described by an instantaneous fluctuation of the isotropic distribution $\delta f_{C}(E, t)$, which is not contained in Eq. (255). We write then

$$
\begin{aligned}
\delta f(\boldsymbol{r}, \boldsymbol{n}, E, t)= & \delta \tilde{f}(\boldsymbol{r}, \boldsymbol{n}, E, t)+\delta f_{C}(E, t), \\
& \boldsymbol{n} \boldsymbol{N}_{n}>0, \quad \mathbf{r} \in \Omega_{n},
\end{aligned}
$$

where $\delta \tilde{f}$ obeys Eq. (255).

The requirement of the conservation of the number of electrons in the cavity leads to minimal correlation $\$ 65$ between $\delta f_{C}(E, t)$ and $\delta \tilde{f}(\boldsymbol{r}, \boldsymbol{n}, E, t)$. The requirement that current is conserved at every instant of time, $\sum_{n} \delta I_{n}=0$, eliminates fluctuations $\delta f_{C}$. After straightforward calculations with the help of Eq. (255) we arrive at the expression 99]

$$
S_{m n}=2 G_{m n} k_{B}\left(T+T_{C}\right), \quad k_{B} T_{C}=\int d E f_{C}\left(1-f_{C}\right) .
$$

It is easy to check that Eq. (257) actually reproduces all the results we have obtained in Section II with the help of the scattering approach. Explicitly, we obtain

$$
k_{B} T_{C}=\frac{e}{2} \sum_{m, n} \beta_{m} \beta_{n}\left(V_{n}-V_{m}\right) \operatorname{coth}\left(\frac{e\left(V_{n}-V_{m}\right)}{2 k_{B} T}\right) .
$$

At equilibrium $T_{C}=T$, and the noise power spectra obey the fluctuation-dissipation theorem. For zero temperature, in the two-terminal geometry we reproduce the noise suppression factor (96); in the multi-terminal case the Hanbury Brown-Twiss results [115], described in Section II, also follow from Eq. 257.

The perceptive reader notices the close similarity of the discussion given above and the derivation of the classical results from the scattering approach invoking a dephasing voltage probe 90,115. (subsection II G). The correlations induced by current conservation are built in in the discussion of the dephasing voltage probe model of the scattering approach. The discussion which we have given

\footnotetext{
${ }^{65}$ Minimal means here that the correlations are minimally necessary - the only non-equilibrium type fluctuations are induced by the current conservation requirement. This kind of fluctuations was discussed by Lax [276]. The approach similar to what we are discussing was previously applied in Ref. 277] to the shot noise in metallic diffusive wires, where it does not work due to the additional fluctuations induced by the random scattering events.
}

above, is equivalent to this approach, with the only difference that fluctuations are at every stage treated with the help of fluctuating distributions. In contrast, the scattering approach with a dephasing voltage probe invokes only stationary, time-averaged distributions.

The comparison of the Boltzmann-Langevin method with the dephasing voltage probe approach also serves to indicate the limitations of the above discussion. In general, for partial dephasing (modeled by an additional fictitious lead) inside the cavity connected via leads with a barrier [115], even the averaged distribution function $f_{C}$ can not be determined simply via Eq. (251): even on the ensemble average, injectivities are not given simply by conductance ratios.

\section{NOISE IN STRONGLY CORRELATED SYSTEMS}

This Section is devoted to the shot noise in strongly correlated systems. This is a rather wide subject due to the diversity of the systems considered. There is no unifying approach to treat strongly correlated systems. Typically, the shot noise in interacting systems is described by methods more complicated than the scattering or Langevin approaches. An attempt to present a detailed description of the results and to explain how they are derived would lead us to the necessity to write a separate review (if not a book) for each subject. Our intention is to avoid this, and below we only present some results for particular systems without an attempt to derive them. The discussion is qualitative; for a quantitative description, the reader is referred to the original works. As a consequence, this Section has the appearance of a collection of independent results.

\section{A. Coulomb blockade}

The term Coulomb blockade is used to describe phenomena which show a blockage of transport through a system due to the electrostatic effects. We recall only some basic facts; the general features of the Coulomb blockade are summarized in the early review article 278. The most common technique to describe Coulomb blockade effects is the master equation approach.

Tunnel barriers. The simplest structure for which one might think that the Coulomb blockade is significant is a tunnel junction. The junction is characterized by a capacitance $C$. From the electrostatic point of view, the system can be regarded as a capacitor where the tunneling between the electrodes is allowed, i.e. the equivalent circuit is the capacitor $C$ connected in parallel with a resistor $R \propto\left(\sum_{n} T_{n}\right)^{-1}$. Due to the additional charging energy $Q^{2} / 2 C$, where $Q$ is the charge of the junction, the current through the junction is blocked (i.e. exponentially small for $k_{B} T \ll e^{2} / C$ ) for voltages below 
$V_{c}=e / 2 C$ (for simplicity, we only consider $V>0$ ). The $I-V$ curve is essentially $I=R^{-1}\left(V-V_{c}\right) \theta\left(V-V_{c}\right)$. The singularity at $V=V_{c}$ is smeared at elevated temperatures and finite resistances (see below). The shot noise is Poissonian for low voltages (since the junction is opaque) and crosses over to the usual $(1-T)$ suppression (for one channel) for high voltages. We note, however, that this description only applies to junctions with $T \ll 1$, and, thus, technically shot noise is Poissonian in the whole range of voltages.

The Coulomb blockade picture given above holds only provided the resistance of the sample is much greater than the quantum of the resistance, $R \gg R_{k} \equiv 2 \pi \hbar / e^{2}$, and breaks down in the opposite limit, where the $I-V$ characteristics are Ohmic and (for zero frequency) insensitive to the charging effects. This latter limit is the subject of work by Ben-Jacob, Mottola and Schön 147 and Schön 27].

Lee and Levitov [28] investigate the effect of the external impedance (active resistance $R_{e x}$ ) on the noise properties of the tunnel junction. They consider the case $R \gg R_{k}$ and describe the crossover between the Ohmic regime $\left(R_{e x} \ll R\right)$ to the Coulomb blockade regime $\left(R_{e x} \gg R\right)$. They also consider finite frequency effects, but do not take into account the displacement currents. They arrive at the remarkable conclusion that the current conservation for noise is violated for high enough frequencies, $(i \omega C)^{-1} \ll|Z(\omega)|$, where $Z$ is the external impedance. Thus, this is an illustration of the statement (Section III) that one has to take care of the displacement currents, even when the scattering matrices are not energy dependent, for frequencies higher than the inverse collective response time, in this particular situation $\left(R_{e x} C\right)^{-1}$.

Quantum dots. An equivalent circuit for a quantum dot with charging (cited in the Coulomb blockade literature as the single-electron transistor, SET) is shown in Fig. 35a. The SET is essentially a two-barrier structure with the capacitances included in parallel to the resistances; in addition, the quantum dot is capacitively coupled to a gate. We assume $R_{1,2} \gg R_{k}$. In the simplest approximation, the role of the gate is simply to fix the number of electrons which are in the dot in the absence of the driving voltage $V, N_{g}=C V_{g} / e, C=C_{1}+C_{2}+C_{g}$. For a moment we assume now $V_{g}=N_{g}=0$. Then for low voltages $V<e / 2 C$ the tunneling is blocked. The point $V=e / 2 C$ is degenerate, since the energies of an empty dot and a dot with one electron are equal. For $e / 2 C<V<3 e / 2 C$ there is one electron in the dot, and so on. Consequently, the $I-V$ curve shows steps (the Coulomb staircase, Fig. 35b). The steps are smeared by the temperature and finite resistance. Actually, the Coulomb staircase is only well pronounced when the dot is asymmetric, $R_{1} \ll R_{2}$, and in addition, $C_{1} \leq C_{2}$. In the opposite case, there is no current until $e / 2 C$, but the $I-V$ characteristics for higher voltages are regular, similarly to the tunnel junction. Finite (fixed) gate voltage shifts the $I-V$ curve; for $N_{g}=1 / 2$ the degeneracy point happens to be at $V=0$, i.e. the system shows Ohmic behavior.

For the case when there is no Coulomb staircase, shot noise was investigated by Belogolovskii and Levin [279]. Based on the master equation approach, they report a shot noise suppression. However the paper is too concise to provide an understanding of the origin of the noise suppression. Independently, for this case shot noise was investigated by Korotkov et al 280 and Korotkov 281] using the master equation approach, and subsequently by Korotkov using the Langevin approach [282]: He adds Langevin sources to the rate equations, each number of electrons in the dot is described by an individual fluctuating source. In this case the shot noise is absent for $V<e / 2 C$, Poissonian at the onset of current (since the tunneling rate through one of the barriers dominates, see below), and for high voltages is described by a Fano factor

$$
F=\frac{R_{1}^{2}+R_{2}^{2}}{\left(R_{1}+R_{2}\right)^{2}} .
$$

The result (258) is genuinely the double-barrier Fano factor (78), since $R_{1,2} \propto T_{1,2}^{-1}$. This high-voltage behavior was independently obtained by Hung and $\mathrm{Wu} 284$ using the Green's function technique. Korotkov 2281] also studied the frequency dependence of shot noise and found that it is a regular function, which for high frequencies $(R C)^{-1} \ll \omega \ll e^{2} / \hbar C, R^{-1}=R_{1}^{-1}+R_{2}^{-1}$, saturates at an interaction-dependent value.

For the general case (Coulomb staircase regime), shot noise was (independently) analyzed by Hershfield et al 283 using a master equation. In the plateau regimes, the transport is via the only state with a fixed number of electrons, and the shot noise is Poissonian (up to exponentially small corrections); in particular, for $V<e / 2 C$ noise is exponentially small. The situation is, however, different close to the degenerate points (at the center of the step of the staircase), since there are now two charge states available. Hershfield et al 283] find that the Fano factor in the vicinity of the degenerate points is $F(V)=\left(\Gamma_{1}^{2}+\Gamma_{2}^{2}\right) /\left(\Gamma_{1}+\Gamma_{2}\right)^{2}$. Here $\Gamma_{1}$ and $\Gamma_{2}$ are the (tunneling) rates to add an electron through barrier 1 and remove an electron through barrier 2. Though this result looks similar to the expression for the resonant double-barrier structure (78), the important difference is that the rates $\Gamma_{1,2}$ are now strongly voltage dependent due to the Coulomb blockade. In particular, at the onset of the step $\Gamma_{1} \ll \Gamma_{2}$, and $F=1$. The Fano factor thus shows dips in the region of the steps. For high voltages the Coulomb blockade is insignificant, and the Fano factor assumes the double-barrier suppression value (258). The resulting voltage dependence of the Fano factor is sketched in Fig. 35b, upper curve. Hershfield et al 283] also perform extensive numerical simulations of shot noise and show that the structure in the Fano factor disappears in the symmetric limit $R_{1} \sim R_{2}$. 


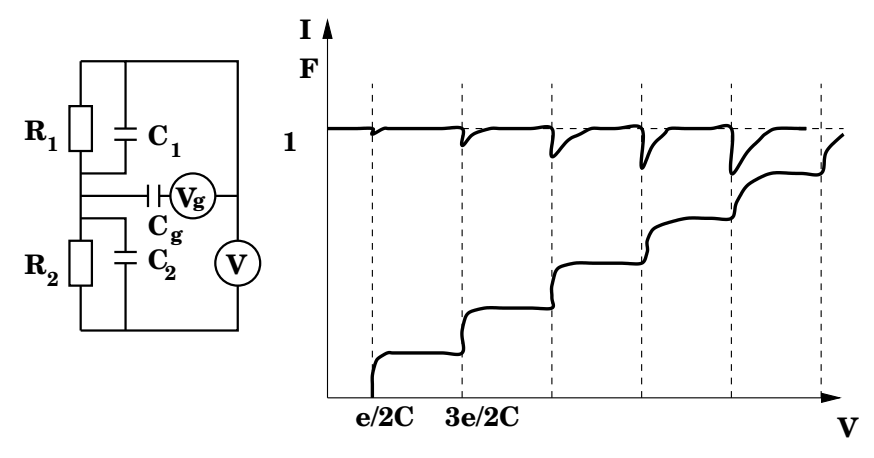

a)

b)

FIG. 35. (a) Equivalent circuit for the single-electron transistor; (b) A sketch of the $I-V$ characteristics (lower curve) and the Fano factor voltage dependence (upper curve) for the very asymmetric case $R_{1} \ll R_{2}, C_{1} \ll C_{2}, V_{g}=0$.

Similar results were subsequently obtained by Galperin et al 285, Hanke et al 286], and Hanke, Galperin, and Chao [287], who also investigate the frequency dependence of shot noise. They point out that the quantity $\partial^{2} S / \partial \omega^{2}$ is more sensitive to the voltage near the Coulomb blockade steps than the noise spectral power $S$. Sub-Poissonian shot noise suppression in the Coulomb blockade regime is numerically confirmed by Anda and Latgé 288, however, at high bias, they find that the average current tends to zero.

Another option is to consider transport properties as a function of the gate voltage $V_{g}$. Both current and noise are periodic functions of $V_{g}$ with the period of $e / C$ (which corresponds to the period of 1 in $N_{q}$ ). As pointed out by Hanke, Galperin, and Chao [287], shot noise is then periodically suppressed below the Poisson value. Wang, Iwanaga, and Miyoshi 289] consider shot noise in the Coulomb blockade regime in a semiconductor quantum dot, where the single-particle level spacing is relatively large. In this situation it is not enough to write the interaction potential in the dot in the form $Q^{2} / 2 C$, and the real space-dependent Coulomb interaction must be taken instead. The current as a function of the gate voltage exhibits a number of well-separated peaks, and, consequently, shot noise is Poissonian everywhere except for the peak positions, where the Fano factor has dips. Surprisingly enough, the numerical results of Wang, Iwanaga, and Miyoshi 289] show that the Fano factor in the dip may be arbitrarily low, certainly below $1 / 2$. They explain this as being due to the suppression of the shot noise by Coulomb interactions.

We also mention here the papers by Krech, Hädicke, and Müller [290] and Krech and Müller [291], who, based on a master equation, conclude that the Fano factor may be suppressed down to zero in the Coulomb blockade regime; in particular, the Fano factor tends to zero for high frequencies. Though we cannot point out an explicit error in these papers, the results seem quite surprising to us. We believe that in the simple model of the Coulomb blockade, when the electrostatic energy is approximated by $Q^{2} / 2 C$, it is quite unlikely that the shot noise is suppressed below $1 / 2$, which is the non-interacting suppression factor for the symmetric double-barrier structure. Clearly, to answer these questions, an analytic investigation, currently unavailable, is required.
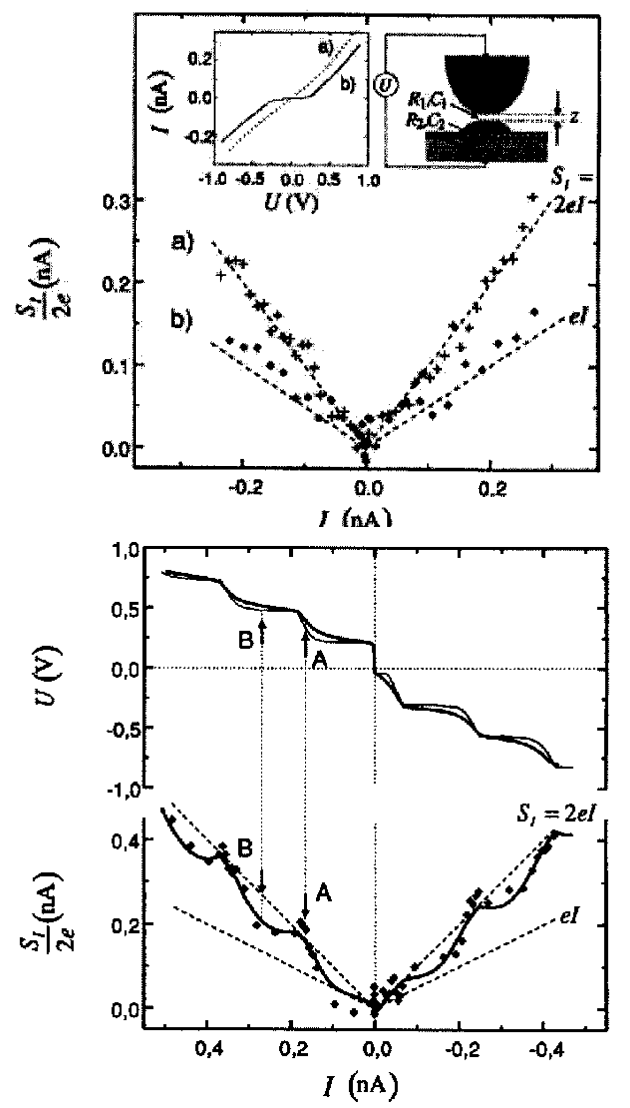

FIG. 36. Experiments by Birk, de Jong, and Schönenberger 30|. Left: shot noise in the quantum dot with $R_{2}=2 R_{1}$ (diamonds); the inset shows the $I-V$ characteristics. Right: $I-V$ curve (top) and shot noise (bottom) in a very asymmetric quantum dot. Solid curves show the theory of Hershfield et al 283. Dashed lines in all cases show the Poisson value $2 e\langle I\rangle$ and the value $e\langle I\rangle$.

Experiments on shot noise in the Coulomb blockade regime were performed by Birk, de Jong, and Schönenberger 30]. They put a nanoparticle between the STM tip and metallic surface, and were able to change the capacitances $C_{1}$ and $C_{2}$ and resistances $R_{1}$ and $R_{2}$. For $R_{2}=2 R_{1}$ they found the "smooth" Coulomb blockade $I-V$ characteristics (no traces of the Coulomb staircase), with the noise crossing over from Poissonian to Eq. 258) with the increasing voltage (Fig. 36, left). In contrast, for the very asymmetric

\footnotetext{
${ }^{66}$ The difference of this setup with a SET is that there is no gate.
} 
case $R_{1} \ll R_{2}$ Birk, de Jong, and Schönenberger [30] observe the Coulomb staircase and periodic shot noise suppression below the Poisson value (Fig. 36, right). They compare their experimental data with the theory of Hershfield et al [283] and find quantitative agreement.

Arrays of tunnel barriers. Consider now a onedimensional array of metallic grains separated by tunnel barriers. This array also shows Coulomb blockade features: The current is blocked below a certain threshold voltage $V_{c}$ (typically higher than the threshold voltage for a single junction), determined by the parameters of the array. Just above $V_{c}$, the transport through the array is determined by a single junction (a bottleneck junction), and corresponds to the transfer of charge $e$. Thus, the shot noise close to the threshold is Poissonian, the Fano factor equals one. With increasing voltage more and more junctions are opened for transport, and eventually a collective state is established in the array [278]: An addition of an electron to the array causes the polarization of all the grains, such that the effective charge transferred throughout the array ${ }^{67}$ is $e / N$, with $N \gg 1$ being the number of grains. In this regime, the Fano factor is $1 / N$. The crossover of shot noise from Poissonian to $1 / N$-suppression was obtained by Matsuoka and Likharev [292], who suggested these qualitative considerations and backed them by numerical calculations.

Other interesting opportunities for research open up if the frequency dependence of shot noise is considered. First, in the collective state these arrays exhibit single-electron tunneling oscillations with the frequency $\omega_{s}=I / e, I$ being the average current. These oscillations are a consequence of the discreteness of the charge transfer through the tunnel barrier. The increase of charge on the capacitor is the result of the development of a dipole across the barrier. This is a polarization process for which the charge is not quantized. However, when the polarization charge reaches $e / 2$ the Coulomb blockade is lifted and the capacitance is decharged by the tunneling transfer of a single electron. Both the continuous polarization process and the discrete charge transfer process give rise to currents in the external circuit. It is difference in time scale for the two processes which causes current oscillations. The polarization process is much slower than the decharging process. The discreteness of the charge transfer process is, as we have seen, a key ingredient needed to generate shot noise. However here, in contrast to the Schottky problem, the charge transfer occurs at time intervals which are clearly not given by a Poisson distribution. Instead, here, ideally the charge transfer process is clocked and there is no shot noise. However in an array of tunnel junctions, there exists the possibility to observe both current oscillations and shot

\footnotetext{
${ }^{67}$ This can be viewed as a transport of solitons with the fractional charge.
}

noise: In an array charge transfer occurs via a soliton (a traveling charge wave). Korotkov, Averin, and Likharev 293 and Korotkov 294] find that arrays of tunnel barriers exhibit both a current that oscillates (on the average) at a frequency $\omega_{s}=I / e$, and shot noise which is strongly peaked at the frequency $\omega_{s}$.

Another type of oscillations are Bloch oscillations, which are due to the translational symmetry of the array. Their frequency is proportional to the voltage drop across each contact; if the array is long and $V \ll N e^{2} / C$, this frequency is approximately $\omega_{B}=e^{2} / 4 \pi \hbar C, \omega_{B} \gg \omega_{s}$. The shot noise also peaks at $\omega_{B}$, as found by Korotkov, Averin, and Likharev [293].

Hybrid structures. Qualitatively, the behavior of a superconducting SET - the same structure as shown in Fig. 35a but with the normal central grain replaced by a superconducting grain - is different from the behavior of the normal SET, since the system is now sensitive to the parity of the number of electrons on the island. If this number is odd, there is always an unpaired electron, and thus the energy of these odd states is shifted up by the superconducting gap $\Delta$ as compared to the even states. Thus, odd states are unfavorable, and for $e^{2} / C, e V<\Delta$, $k_{B} T \ll \Delta$ cannot be occupied. This parity effect 295. is superimposed on the usual Coulomb blockade behavior, and the general picture can be very complex. In particular, in the limiting case $e^{2} / C \ll \Delta$ and for $N_{g}=0$, we anticipate the following behavior of noise. For $V<e / C$ the current is Coulomb blocked, and the shot noise is thus Poissonian. To determine the Fano factor in this case, we must find the processes which give rise to current slightly above the threshold $V_{c}=e / C$. The dominant process is an electron coming from a reservoir which is Andreev reflected at the interface, and generates a Cooper pair in the superconducting grain, which is again converted to an electron and a hole pair at the interface. This process results in the transfer of an effective charge of $2 e$; the Fano factor is thus 2. For $e^{2} / C<e V \ll \Delta$, the Fano factor is expected to be smaller as the voltage is increased and the Coulomb blockade is lifted. For $\mathrm{eV} \sim \Delta$ singleelectron tunneling starts to play a role; eventually, for $e V \gg \Delta$ shot noise is determined by the double-barrier value of the Fano factor (258).

With the help of the gate, the polarization charge can be adjusted to be $N_{g}=1$. In this case, the system is at the degeneracy point, since the energies of two ground states with an even number of carriers are the same. The current is then finite for any bias [296], and there is no Coulomb blockade behavior: Cooper pair tunneling starts from zero voltage.

These considerations are qualitative, and must be confirmed by quantitative calculations. Presently, there are not many results available on noise in interacting hybrid structures. The investigations of shot noise in SNS and NSN systems in the Coulomb blockade regime were pioneered by Krech and Müller [297]. They use the same master equation technique as in their previous works 290,291, and arrive at the same unphysical result that 
the shot noise is always suppressed for high frequencies down to zero.

Hanke et al 298,299 perform numerical calculations of the shot noise in superconducting SETs in the regime $e^{2} / C<\Delta, N_{g}=1$. In this case, there are several threshold voltages, and the Fano factor is found to peak at each threshold. Strangely, Refs. 298,299 find that the Fano factor may lie well above 2 . We do not currently understand this result.

Ferromagnetic junctions. Recently, junctions in which one or several electrodes are ferromagnetic have become of interest in mesoscopic physics. Many different structures have been proposed and, in principle, one may discuss shot noise for all these structures; presently, only one theoretical and one experimental paper exist.

Bułka et al 300] consider a structure in which two electrodes are separated by a quantum dot in the Coulomb blockade regime (a ferromagnetic analog of the SET). They assume that there is no spin-flip scattering in the dot. The difference between the two spin polarizations in the electrodes is phenomenologically modeled as a difference in resistances: Thus, if the ferromagnetic reservoir 1 is spin-up polarized, the resistance $R_{1 \downarrow}$ for spin-down electrons is much higher than the resistance $R_{1 \uparrow}$ for spinup electrons. Assuming that all the resistances are much higher than $R_{k}$, Butka et al [300] generalize the master equation approach to the case of spin-dependent transport, and present results of a numerical calculation.

In structures in which both electrodes are ferromagnetic (FNF junction), the orientation of the magnetic moments of the electrodes becomes important. To understand what happens, we discuss first the shot noise suppression in a non-interacting FNF junction 301. We have already seen that it is relevant at the steps of the Coulomb staircase. We assume that both electrodes are made of the same ferromagnetic material, so that the ratio of the resistances for spin-up and spin-down propagation is the same. The junction is also assumed to be very asymmetric. Thus, for the parallel orientation (when the magnetic moments of both reservoirs are spinup), we have the resistances $R_{1 \uparrow}=R_{0}, R_{1 \downarrow}=\alpha R_{0}$, $R_{2 \uparrow}=\beta R_{0}$, and $R_{2 \downarrow}=\alpha \beta R_{0}$. The constants $\alpha$ and $\beta$ describe the asymmetry of the spin-up and spin-down propagation, and the asymmetry of the barrier, respectively. For a given spin projection, the current is proportional to $R_{1} R_{2} /\left(R_{1}+R_{2}\right)$, while the shot noise is proportional to $R_{1} R_{2}\left(R_{1}^{2}+R_{2}^{2}\right) /\left(R_{1}+R_{2}\right)^{3}$. Here $R_{1}$ and $R_{2}$ must be taken for each spin projection separately; the total current (shot noise) is then expressed as the sum of the currents (shot noises) of spin-up and spin-down electrons. Evaluating in this way the Fano factor, we obtain

$$
F_{\uparrow \uparrow}=\frac{1+\beta^{2}}{(1+\beta)^{2}},
$$

which is precisely the double-barrier Fano factor 78, since the Fano factors for spin-up and spin-down electrons are the same.
Similarly, for the anti-parallel $(\uparrow-\mathrm{N}-\downarrow)$ orientation we write $R_{1 \uparrow}=R_{0}, R_{1 \downarrow}=\alpha R_{0}, R_{2 \uparrow}=\alpha \beta R_{0}$, and $R_{2 \downarrow}=$ $\beta R_{0}$. The Fano factor is

$$
F_{\uparrow \downarrow}=\left(\frac{1+\alpha^{2} \beta^{2}}{(1+\alpha \beta)^{3}}+\frac{\alpha^{2}+\beta^{2}}{(\alpha+\beta)^{3}}\right)\left(\frac{1}{1+\alpha \beta}+\frac{1}{\alpha+\beta}\right)^{-1},
$$

and depends now on $\alpha$. In the strongly asymmetric junction, $\alpha, \beta \gg 1$, the Fano factor for the parallel orientation (259) becomes 1. At the same time, for the anti-parallel orientation Eq. (260) is entirely determined by spin-down electrons, and takes the form $F_{\uparrow \downarrow}=\left(\alpha^{2}+\beta^{2}\right) /(\alpha+\beta)^{2}$. Thus, the shot noise in the anti-parallel configuration is suppressed as compared to the parallel case. This situation is, of course, the same in the Coulomb blockade regime, and this is precisely what Bułka et al 300] find numerically.

In these discussions, it is assumed that both spin channels are independent. In reality, spin relaxation processes which couple the two channels might be important.

It is interesting to consider the structure in which one of the reservoirs is ferromagnetic and the another one is normal (FNN junction). Bułka et al [300] only treat the limiting case when, say, spin-down electrons cannot propagate in the ferromagnet at all $\left(R_{1 \downarrow}=\infty\right)$. They find that the $I-V$ curve in this case shows negative differential resistance, and shot noise may be enhanced above the Poisson value, similarly to what we have discussed in Section \ for quantum wells.

Experimentally, the main problem in ferromagnetic structures is to separate shot noise and $1 / f$-noise. Nowak, Weissman, and Parkin 302 measured the lowfrequency noise in a tunnel junction with two ferromagnetic electrodes separated by an insulating layer. They succeeded in extracting information on shot noise, and report sub-Poissonian suppression for low voltages, but they did not perform a systematic study of shot noise, and the situation, both theoretically and experimentally, is very far from being clear.

Concluding remarks on the Coulomb blockade. A patient reader who followed this Review from the beginning has noticed that we have considerably changed the style. Indeed, in the previous Sections we mostly had to deal with results, which are physically appealing, well established, cover the field, and in many cases are already experimentally confirmed. Here, instead, the results are contradictory, in many cases analytically unavailable, and, what is more important, fragmentary they do not systematically address the field. To illustrate this statement, we only give one example; we could have cited dozens of them. Consider a quantum dot under low bias; it is typically Coulomb blocked, unless the gate voltage is tuned to the degenerate state, so that $N_{g}$ is half-integer. As we have discussed above, the current dependence on the gate voltage is essentially a set of peaks, separated by the distance of approximately $e / C$. 
Between the peaks (in the valleys), as we implicitly assumed, the zero-temperature current is due to quantum tunneling, and is exponentially small. We concluded therefore, that the Fano factor is 1 - the shot noise is Poissonian. In reality, however, there are cotunneling processes - virtual transitions via the high-lying state in the dot - which are not exponentially suppressed and thus give the main contribution to the current. Cotunneling is a genuinely quantum phenomenon, and cannot be obtained by means of a classical approach. It is clear that the cotunneling processes may modify the Fano factor in the valleys; moreover, it is a good opportunity to study quantum effects in the shot noise. This problem, among many others, remains unaddressed 8 .

\section{B. Anderson and Kondo impurities}

Anderson impurity model. In the context of mesoscopic physics, the Anderson impurity model describes a resonant level with a Hubbard repulsion. It is sometimes taken as a model of a quantum dot. Commonly the entire system is described by the tight-binding model with non-interacting reservoirs and an interaction $U \hat{n}_{\downarrow} \hat{n}_{\uparrow}$ on the site $i=0$ (resonant impurity), with $\hat{n}_{\downarrow}$ and $\hat{n}_{\uparrow}$ being the operators of the number of electrons on this site with spins down and up, respectively. Tunneling into the dot is described, as in the non-interacting case, by partial tunneling widths $\Gamma_{L}$ and $\Gamma_{R}$, which may be assumed to be energy independent. The on-site repulsion is important (in the linear regime) when $U \gg E_{0}$, where $E_{0}$ is the energy of the resonant impurity relative to the Fermi level in the reservoirs. In this case, for low temperatures $T<T_{K}$ the spin of electrons traversing the quantum dot starts to play a considerable role, and the system shows features essentially similar to the Kondo effect 304,305]. Here $T_{K}$ is a certain temperature, which is a monotonous function of $U$, and may be identified with the effective Kondo temperature. For $T<T_{K}$ the effective transmission coefficient grows as the temperature is decreased, and for $T=0$ reaches the resonant value $T^{\max }=4 \Gamma_{L} \Gamma_{R} /\left(\Gamma_{L}+\Gamma_{R}\right)^{2}$ (see Eq. (75)) for all impurities which are closer to the Fermi surface than $\Gamma_{L}+\Gamma_{R}$. Subsequent averaging over the impurities [304] gives rise to the logarithmic singularity in the conductance for zero temperature. A finite bias voltage $V$ smears the singularity [304,306]; thus, the differential conductance $d I / d V$ as a function of bias shows a narrow peak around $V=0$ and two broad side peaks for $e V= \pm U$.

\footnotetext{
${ }^{68} \mathrm{~A}$ more complicated problem - transport through the double quantum dot in the regime when the cotuneling dominates - was investigated by Loss and Sukhorukov 303 with the emphasis on the possibility of probing entanglement (see Appendix $B$. They report that the Fano factor equals one.
}

Theoretical results on shot noise are scarce. Hershfield 307 performs perturbative analysis in powers of $U$ of the shot noise, based on the Green's function approach. His results are thus relevant for the high-temperature regime, but do not describe the Kondo physics, which is non-perturbative in $U$. He finds that the noise, apart from the non-interacting contribution (described by the Fano factor (78)) contains also an interacting correction. This correction is a non-trivial function of the applied bias voltage; it is always positive for a symmetric barrier $\Gamma_{L}=\Gamma_{R}$, but may have either sign for an asymmetric barrier. This interacting correction is zero for zero bias, and peaks around a certain energy $E_{r}$, which is the bare resonant energy $E_{0}$, renormalized by interactions. For higher voltages it falls off with energy, and in the limit $e V \gg U, E_{0}$ the Fano factor returns to the noninteracting value (78). Thus, in this case the interactions may either enhance or suppress the noise. We also point out the analogy with the Coulomb blockade results: In the symmetric case $R_{1}=R_{2}$, the actual Coulomb blockade noise suppression is always stronger, than the noninteracting suppression (258); generally, it may be either stronger or weaker. Yamaguchi and Kawamura [308,309] perform a complementary analysis by treating the tunneling Hamiltonian perturbatively. They find that the shot noise is strongly suppressed as compared with the Poisson value for voltages $e V \sim E_{0}$ and $e V \sim E_{0}+U$ (the latter resonance corresponds to the addition of the second electron to the resonant state, which is then shifted upwards by $U$ ).

For the Kondo regime, we expect that, since the effective transmission coefficient tends to $T^{\max }$ for zero temperature, the shot noise is a sensitive function of $k_{B} T$, which for a symmetric barrier decreases and eventually vanishes as the temperature tends to zero. This regime is investigated by Ding and $\mathrm{Ng}$ [310], who complete the Green's functions analysis by numerical simulations. They only plot the results for the symmetric case $\Gamma_{L}=\Gamma_{R}$ and only for $T=T_{K}$; the shot noise in this regime is, indeed, suppressed below the non-interacting value for any applied bias. Yamaguchi and Kawamura [309], treating the tunneling Hamiltonian perturbatively, report that the Fano factor is suppressed down to zero at zero bias. Results concerning averages over impurities are unavailable.

Kondo model. In mesoscopic physics, this is the model of two non-interacting reservoirs which couple to the $1 / 2$-spin in the quantum dot via exchange interaction. The interacting part of the Hamiltonian is $\hat{H}_{\text {int }}=J_{\lambda}^{\alpha \beta} s_{\alpha \beta}^{\lambda} \sigma^{\lambda}$, where $\lambda=x, y, z$ and $\alpha, \beta=L, R$. Here $s_{\alpha \beta}^{\lambda}$ are the matrix elements of the electron spin operator in the basis of the reservoir states, and $\sigma^{\lambda}$ is the spin of the Kondo impurity. In physical systems the coupling $J_{\lambda}$ is symmetric; however, to gain some insight and use the exact solutions, other limits are often considered.

To our knowledge, the only results on shot noise in the Kondo model are due to Schiller and Hershfield [311], 
who consider a particular limiting case (Toulouse limit), $J_{z}^{\alpha \beta}=J_{y}^{\alpha \beta}, J_{z}^{L R}=J_{z}^{R L}=0$, and $J_{z}^{L L}=J_{z}^{R R}$. As a function of the bias voltage, the Fano factor is zero at zero bias, and grows monotonically. In the high-bias limit the noise is Poissonian rather than suppressed according to Eq. (78). The transport properties of the Kondo model are strongly affected by an applied magnetic field, which may drive the Fano factor well above the Poisson value. The frequency dependence of the shot noise is sensitive to the spectral function of the Kondo model, and exhibits structure at the inner scales of energy. The studies [311], though quite careful, do not, of course, exhaust the opportunities to investigate shot noise in strongly correlated systems, offered by the Kondo model.

\section{Tomonaga-Luttinger liquids and fractional quantum Hall edge states}

Many problems concerning (strictly) one-dimensional systems of interacting electrons may be solved exactly by using specially designed techniques. As a result, it turns out that in one dimension, electron-electron interactions are very important. They lead to the formation of a new correlated state of matter, a Tomonaga-Luttinger liquid, which is characterized by the presence of gapless collective excitations, commonly referred as plasmons. In particular, the transport properties of the one-dimensional wires are also quite unusual. We only give here the results which we subsequently use for the description of noise; a comprehensive review may be found e.g. in Ref. 312. Throughout the whole subsection we assume that the interaction is short-ranged and one-dimensional, $V\left(x-x^{\prime}\right)=V_{0} \delta\left(x-x^{\prime}\right)$, and the voltage $V>0$.

For an infinite homogeneous Luttinger liquid the "conductance" is renormalized by interactions, $G=g e^{2} / 2 \pi \hbar$, where the dimensionless interaction parameter,

$$
g=\left(1+\frac{V_{0}}{\pi \hbar v_{F}}\right)^{-1 / 2}
$$

will play an important role in what follows. This parameter equals 1 for non-interacting electrons, while $g<1$ for repulsive interactions. However, if one takes into account the reservoirs, which corresponds to a proper definition of conductance, the non-interacting value $e^{2} / 2 \pi \hbar$ is restored, and thus the interaction constant $g$ cannot be probed in this way.

If one has an infinite system (no reservoirs) with a barrier 69 , the situation changes. Even an arbitrarily weak barrier totally suppresses the transmission in the interacting case $g<1$, and for zero temperature there is no linear dc conductance. The two limiting cases may be

\footnotetext{
${ }^{69}$ This barrier is routinely called "impurity" in the literature.
}

treated analytically. For strong barriers (weak tunneling), when the transmission coefficient is $T \ll 1$, the $I-V$ curve in the leading order in $V$ is

$$
\langle I\rangle=\frac{e^{2}}{2 \pi \hbar} a T V^{2 / g-1},
$$

where $a$ is a non-universal (depending on the upper energy cut-off) constant. In the opposite case of weak reflection, $1-T \ll 1$, the interactions renormalize the transmission coefficient, so that the barrier becomes opaque, and for low voltages we return to the result (262). On the other hand, for high voltages the backscattering may be considered as a small correction, and one obtains

$$
\langle I\rangle=\frac{g e^{2}}{2 \pi \hbar} V-\frac{e^{2}}{2 \pi \hbar} b T V^{2 g-1},
$$

where $b$ is another non-universal constant. Eq. (263) is only valid when the second term on the right-hand side is small. To emphasize the difference, we will refer to the weak and strong tunneling cases (which describe the regimes (262) and (263), respectively), rather than to the cases of transparent and opaque barriers. In particular, whatever the strength of the barrier, for low voltages and temperatures the tunneling is weak 70 .

For the transmission through a double-barrier structure resonant tunneling may take place, but the resonances become infinitely narrow in the zero-temperature limit.

What is extremely important for the following is that weak tunneling is accompanied by a transfer of charge $g e$ between left- and right-moving particles (we can loosely say that there are quasiparticles with the charge ge which are scattered back from the barrier), while in the case of the strong tunneling the charge transfer across the barrier is $e$ - there is tunneling of real electrons.

Whereas the Luttinger liquid state may, in principle, be observed in any one-dimensional system, the most convenient opportunity is offered by the fractional quantum Hall effect (FQHE) edge states. Indeed, the edge state of a sample in the FQHE regime is a onedimensional system, and it may be shown that for the bulk filling factor $\nu=1 /(2 m+1), m \in \mathcal{Z}$ (Laughlin states), the interaction parameter $g$ takes the same value $g=1 /(2 m+1)$. The difference with the ordinary Luttinger liquid is that the FQHE edge states are chiral: the motion along a certain edge is only possible in one direction. Thus, if we imagine a FQHE strip, the electrons along the upper edge move, say, to the right, and the

\footnotetext{
${ }^{70}$ For the FQHE case (see below) this should not cause any confusion: Strong tunneling regime means strong tunneling through the barrier, which is the same as weak backscattering, or weak tunneling of quasiparticles between the edge states. Conversely, the weak tunneling regime means that the edge states are almost not interconnected (Fig. 37).
} 
electrons along the lower edge move to the left. For the transport properties we discuss this plays no role 11 , and the expressions (262) and (263) remain valid. a)

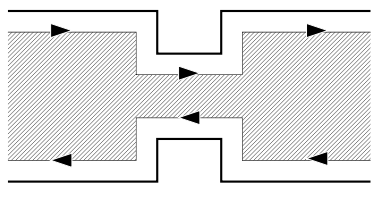

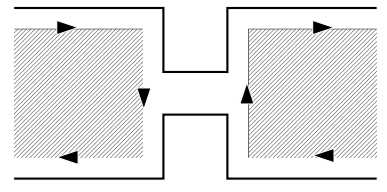

b)
FIG. 37. The tunneling experiment with the FQHE edge states: (a) strong tunneling; (b) weak tunneling. The shaded areas denote the location of the FQHE droplet(s).

In particular, for the FQHE case the quasiparticles with the charge $\nu e$ may be identified with the Laughlin quasiparticles. The distinction between the strong and weak tunneling we described above also gets a clear interpretation (Fig. 37), which is in this form due to Chamon, Freed and Wen 313. Indeed, consider a FQHE strip with a barrier. If the tunneling is strong (Fig. 37a), the edge states go through the barrier. The backscattering corresponds then to the charge transfer from the upper edge state to the lower one, and this happens via tunneling between the edge states inside the FQHE strip. Thus, in this case, there are Laughlin quasiparticles which tunnel. In principle, the electrons may also be backscattered, but such events have a very low probability (see below). In the opposite regime of weak tunneling, the strip splits into two isolated droplets (Fig. 37b). Now the tunneling through the barrier is again the tunneling between two edge states, but it only may happen outside the FQHE state, where the quasiparticles do not exist. Thus, in this case, one has tunneling of real electrons.

Theory of dc shot noise. Shot noise in Luttinger liquids was investigated by Kane and Fisher 314 using the bosonization technique ${ }^{22}$. The conclusion is that for an ideal infinite one-dimensional system there is no shot noise ${ }^{73}$. Shot noise appears once the barrier is inserted. For the strong tunneling regime the shot noise is

$$
S=2 g e\left(\frac{g e^{2}}{2 \pi \hbar} V-\langle I\rangle\right),
$$

where the average current $\langle I\rangle$ is given by Eq. (263) in the regime in which the latter is valid. If we introduce the (small) backscattering current $I_{b}=\left(e^{2} / 2 \pi \hbar\right) b T V^{2 g-1}$, the shot noise is written as

\footnotetext{
${ }^{71} \mathrm{~A}$ difference for shot noise is that any experiment with the FQHE edge states is always four-terminal. The behavior of all correlation functions which we discuss below is the same.

${ }^{72}$ Ref. [314] also considers the finite-temperature case and describes the crossover between thermal and shot noise.

${ }^{73}$ The same is, of course, also true for a system between the two reservoirs.
}

$$
S=2 g e I_{b}
$$

which physically corresponds to the Poisson backscattering stream of (Laughlin) quasiparticles with the charge ge. Eq. (265) is precisely the analog of the two-terminal expression $S=\left(e^{3} V / \pi \hbar\right) T(1-T)$ for the non-interacting case, which may be written as $S=2 e I_{b}$ for $1-T \ll 1$ and corresponds to a Poissonian distributed stream of backscattered electrons. As we have mentioned already, there is also a contribution to the shot noise (264) due to the tunneling of electrons; as explicitly shown by Auerbach 315, this contribution is exponentially suppressed. Thus, the shot noise experiments in the strong tunneling regime open the possibility to measure the fractional charge. This possibility has been experimentally realized (see below).

For the case of weak tunneling, the shot noise is Poissonian with the charge $e, S=2 e\langle I\rangle$ : It is determined by the charge of tunneling electrons. Expressions for noise interpolating between this regime and Eq. (264), as well as a numerical evaluation for $g=1 / 3$, are provided by Fendley, Ludwig, and Saleur 316]; Fendley and Saleur 317 and Weiss 318 generalize them to finite temperatures.

For the resonant tunneling process, at resonance, the shot noise is given by 314

$$
S=4 g e\left(\frac{g e^{2}}{2 \pi \hbar} V-\langle I\rangle\right)=4 g e I_{b},
$$

which corresponds to the effective charge $2 \mathrm{eg}$. This reflects the fact that in resonance the excitations scatter back in pairs. Safi 319] argues, however, that the contribution due to the backscattering of single quasiparticles is of the same order; this statement may have implications for the Fano factor, which is then between $g$ and $2 g$.

Sandler, Chamon, and Fradkin [320] consider a situation in the strong tunneling regime with a barrier separating the two FQHE states with different filling factors $\nu_{1}=1 /\left(2 m_{1}+1\right)$ and $\nu_{2}=1 /\left(2 m_{2}+1\right)$. One of the states may be in the integer quantum Hall regime, for instance $m_{1}=0$. In particular, the case of $\nu_{1}=1$ and $\nu_{2}=1 / 3$ may be solved exactly. They conclude that the noise in this system corresponds to a Poissonian stream (265) of backscattered quasiparticles which are now, however, not the Laughlin quasiparticles of any of the two FQHE states. The charge of these excitations, which is measured by the shot noise, is $\tilde{g} e$, with $\tilde{g}^{-1}=\left(\nu_{1}^{-1}+\nu_{2}^{-1}\right) / 2=m_{1}+m_{2}+1$. Thus, for $\nu_{1}=1$ and $\nu_{2}=1 / 3$ the effective charge is $e / 2$. This also implies that the shot noise experiment cannot distinguish certain combinations of filling factors: the effective charge of the $1 / 3-1 / 3$ junction is the same as that of the $1-1 / 5$ junction.

We also note that the above results were obtained for infinite wires (or FQHE edges). Taking into account the electron reservoirs, as we have mentioned above, changes 
the conductance of an ideal wire. However, it is not expected to affect the Fano factor of a wire with a barrier, which is determined by the scattering processes at the barrier. On the other hand, Ponomarenko and Nagaosa 321] present a calculation which implies that the shot noise in the wire connected to reservoirs is Poissonian with the charge $e$. In our opinion, this statement is not physically appealing, and we doubt that it is correct. Nevertheless, it deserves a certain attention, and more work is needed in this direction.

Frequency-dependent noise. The frequency dependence of noise was studied by Chamon, Freed, and Wen 322, 313, who first derived results perturbative in the tunneling strength, and subsequently were able to find an exact solution for $g=1 / 2$. Lesage and Saleur [323,324 and Chamon and Freed 325] developed non-perturbative techniques valid for any $g$. We briefly explain the main results, addressing the reader for more details to Refs. 313,323. The frequency dependence of noise is essentially similar to that for non-interacting electrons in the case when the scattering matrices are energy independent. There is the $|\omega|$ singularity for zero frequency; the singularity itself is not changed by the interaction, but the coefficient in front of this singularity is interactionsensitive 4 . Furthermore, there is a singularity at the frequency $\omega=e V / \hbar$, which corresponds to the motion of electrons. One could expect that the role of interactions consists in the creation of yet one more singularity at the "Josephson" frequency $\omega_{J}=g e V / \hbar$, reflecting the quasiparticle motion. Indeed, as shown by Chamon, Freed, and Wen [313], shot noise exhibits structure at this frequency. This structure is, however, smeared by the finite tunneling probability, and does not represent a true singularity 75 . Lesage and Saleur 323 also predict some structure at the multiple Josephson frequency $n g \mathrm{eV} / \hbar$, which could be a clear signature of interactions.

FQHE: Other filling factors. An interesting question is what happens for the edge states in the FQHE regime with filling factors different from $1 /(2 m+1)$. In this case, the structure of edge states is more complicated. Although currently there is no general accord on the precise form of this structure, it is clear that for the non-Laughlin FQHE states there are two or several edge states, propagating in the same or opposite directions. Furthermore, it is not quite clear whether different assumptions for the edge structure would lead to different

\footnotetext{
${ }^{74}$ There is a discrepancy in the literature concerning this issue: Chamon, Freed, and Wen [322] and Chamon and Freed [325] report that the coefficient in front of $|\omega|$ is proportional to $V^{4(g-1)}$, while Lesage and Saleur find that the singularity is $V^{4(1-1 / g)}$. The reason for this discrepancy is currently unclear.

${ }^{75}$ In the perturbative calculation it appears as a true singularity, though. Chamon, Freed and Wen [313] call this a "fake singularity".
}

predictions for the shot noise. If this is the case, the shot noise measurements can be used to test the theories of the structure of the FQHE edge.

Imura and Nomura 326 apply a Luttinger liquid description for the investigation of shot noise in the FQHE plateau regime with the filling factor $\nu=2 /(2 m \pm 1), m \in$ $\mathcal{Z}$. They predict that, as the gate voltage which forms the barrier changes, the conductance of the sample crosses from the plateau with the value $(1 /(m \pm 1))\left(e^{2} / 2 \pi \hbar\right)$ to another plateau $(2 /(2 m \pm 1))\left(e^{2} / 2 \pi \hbar\right)$. These expressions apply in the weak scattering regime, and the full Poissonian noise is determined by the effective charges $q=e /(m \pm 1)$ and $q=e /(2 m \mp 1)$ (rather than $\nu e)$ at the first and second plateaus, respectively. Their results are in agreement with the experimental data for $\nu=2 / 5$, and also with the picture emerging from the phenomenological consideration of transport in the composite fermion model (see below).

Fractional statistics. After we have shown that the shot noise experiments with the FQHE edge states may probe fractional charge of Laughlin quasiparticles, one may naturally ask the question: Can the fractional statistics, which the Laughlin quasiparticles are known to obey, also be probed in the shot noise experiments? This problem was addressed by Isakov, Martin and Ouvry [327], who consider the two-terminal experiment for the independent charged particles obeying the exclusion statistics. They obtain that the crossover between zerotemperature shot noise and Nyquist noise is sensitive to the statistics of quasi-particles. We believe that the paper 327 has a number of serious drawbacks. To start with, the statistical particle counting arguments which the paper takes as a departing point, are unable to reproduce the exact results which follow from the scattering matrix approach for bosons, and thus had to add ad hoc certain terms to reproduce these results in the limiting case. Then, the exclusion statistics apply to an ensemble of particles and not to single particles; it is not clear whether the notion of independent particles obeying the exclusion statistics is meaningful. Finally, in the scattering problem one needs to introduce the reservoirs, which are not clearly defined in this case. Having said all this, we acknowledge that the question, which Isakov, Martin and Ouvry [327] address, is very important. Presumably to attack it one must start with the ensemble of particles; we also note that the effects of statistics are best probed in the multi-terminal geometry rather that in the two-terminal case. A demonstration of the HBT-type effect with the FQHE edge states would clearly indicate the statistics of the quasiparticles.

Experiments. Saminadayar et al [328] and, independently, de-Picciotto et al 329] performed measurements on a FQHE strip with the filling factor $\nu=1 / 3$ into which they inserted a quantum point contact. The transmission coefficient of the point contact can be modified by changing the gate voltages. In particular, Saminadayar et al 328] obtained the data showing the crossover from strong tunneling to the weak tunneling regime. Their 
results, shown in Fig. 38, clearly demonstrate that in the strong tunneling (weak backscattering) regime the effective charge of the carriers is $e / 3$. As the tunneling becomes weak, the effective charge crosses over to $e$, as expected for the strong backscattering regime. They also carefully checked the crossover between the shot noise and the equilibrium noise, and found an excellent agreement with the theory.

Though the results of Refs. [328] and 329] basically coincide, we must point out an important difference which is presently not understood. The transmission in the experiment by de-Picciotto et al 329] is not entirely perfect: they estimate that for the sets of data they plot the transmission coefficients are $T=0.82$ and $T=0.73$. Taking this into account, they phenomenologically insert the factor $T$ in the expression for the shot noise and fit the data to the curve $S=(2 e / 3) T I_{B}$. In this way, they obtain a good agreement between the theory and experiment. On the other hand, Saminadayar et al [328] fit their data to the curve $S=(2 e / 3) I_{B}$ (without the factor $T$ ). An attempt to replot the data taking the factor $T$ into account leads to an overestimate of the electron charge. The theory we described above predicts a more complicated dependence than just the factor $T$; therefore it may be important to clarify this detail in order to improve our understanding of the theory of FQHE edge states.

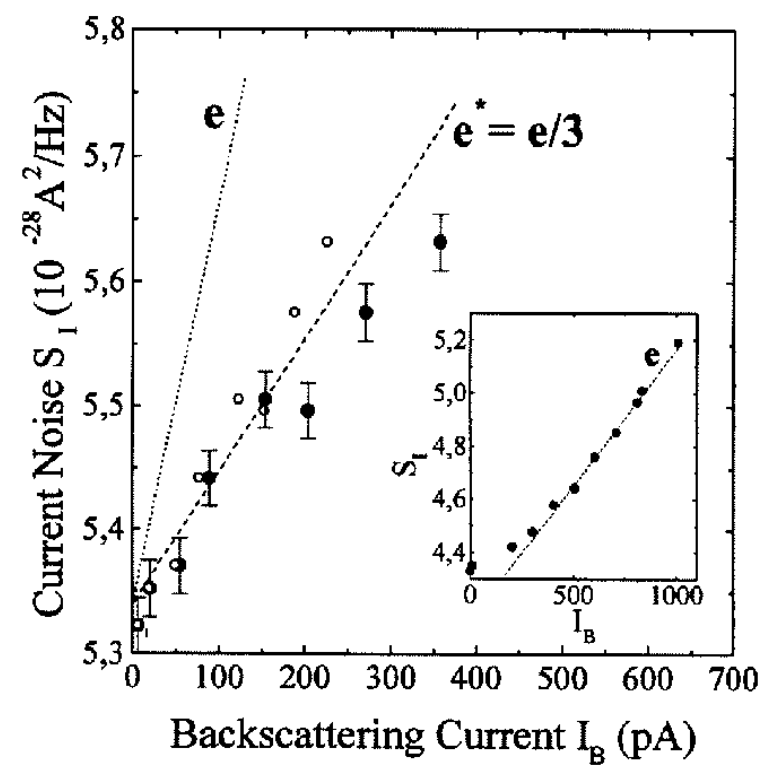

FIG. 38. Experimental results of Saminadayar et al [328] for $\nu=1 / 3$ (strong tunneling - weak backscattering regime).

Reznikov et al 330 performed similar measurements in the magnetic field corresponding to the filling factor $\nu=2 / 5$. Changing the gate voltage (and thus varying the shape of the quantum point contact) they have observed two plateaus of the conductance, with the heights $(1 / 3)\left(e^{2} / 2 \pi \hbar\right)$ and $(2 / 5)\left(e^{2} / 2 \pi \hbar\right)$, respectively. The noise measurements showed that the effective charges at these plateaus are $e / 3$ and $e / 5$, respectively. These results are in agreement with the subsequent theory of Imura and Nomura 326], and also with the predictions of the composite fermion model (see below): They assumed that there are two transmission channels which in turn open with the gate voltage. This experiment is important since it clearly shows that what is measured in the FQHE shot noise experiments is not merely a filling factor (like one could suspect for $\nu=1 / 3$ ), but really the quasiparticle charge.

\section{Composite fermions}

An alternative description of the FQHE systems is achieved in terms of the composite fermions. Starting from the FQHE state with the filling factor $\nu=$ $p /(2 n p \pm 1), n, p \in \mathcal{Z}$, one can perform a gauge transformation and attach $2 n$ flux quanta to each electron. The resulting objects (an electron with the flux attached) still obey the Fermi statistics and hence are called composite fermions (CF). The initial FQHE state for electrons corresponds in the mean field approximation to the filling factor $\tilde{\nu}=p$ for $\mathrm{CF}$, i.e. the composite fermions are in the integer quantum Hall regime with the $p$ Landau levels filled. In particular, the half-filled Landau level corresponds to the $\mathrm{CF}$ in zero magnetic field. Composite fermions interact electrostatically via their charges, and also via the gauge fields, which are a measure of the difference between the actual flux quanta attached and the flux treated in the mean field approximation. It is important that, at least in the mean field approximation, the composite Fermions do not form a strongly interacting system, and therefore may be regarded as independent particles. One can then proceed by establishing an analogy with the transport of independent or weakly interacting electrons.

Von Oppen 331] considers the shot noise of composite fermions at the half-filled Landau levels, assuming that the sample is disordered. He develops a classical theory based on the Boltzmann-Langevin approach ${ }^{76}$ (see Section VI), incorporating interactions between them. Though the fluctuations of both electric and magnetic fields now become important, in the end he obtains the same result as for normal diffusive wires: In the regime of negligible interactions between the CF's, the Fano factor equals $1 / 3$ and is universal. Likewise, in the regime when the $\mathrm{CF}$ distribution function is in local equilibrium (ana$\log$ of the hot electron regime), the suppression factor is $\sqrt{3} / 4$.

\footnotetext{
${ }^{76}$ To this end, he has to assume implicitly the existence of two CF reservoirs, described by equilibrium Fermi distribution functions.
} 
Kirczenow 332 considers current fluctuations in the FQHE states, appealing to statistical particle counting arguments. However, he does not take into account any kind of scattering, and only treats equilibrium (Nyquist) noise for which the result is known already.

Shot noise in the FQHE strip $(\nu=2 /(2 p+1))$ with a tunnel barrier was discussed by de Picciotto 333 who assumed that the composite Fermions are transmitted through the quantum point contact similarly to the noninteracting electrons. Namely, there are $p$ channels corresponding to the $p$ CF Landau levels. Each channel is characterized by an individual transmission coefficient. As the gate voltage is changed the channels open (the corresponding transmission coefficient crosses over from 0 to 1$)$, and the conductance exhibits plateaus with the height $\left.\left(e^{2} / 2 \pi \hbar\right) l /(2 l+1), 1<l<p\right)$. The shot noise at the plateau $l$ is then given as the Poisson backscattered current with the effective charge $e /(2 l+1)$. Though this paper is phenomenological and requires further support from microscopic theory, we note that all the features predicted in Ref. 333] are not only in agreement with the Luttinger liquid approach by Imura and Nomura [326], but also were observed experimentally [330 for $\nu=2 / 5$ (see above). In this case there are two channels corresponding to $p=1$ and $p=2$, which implies conductance plateaus with heights $(1 / 3)\left(e^{2} / 2 \pi \hbar\right)$ and (for higher gate voltage) $(2 / 5)\left(e^{2} / 2 \pi \hbar\right)$; the corresponding charges measured in the shot noise experiment are $e / 3$ and $e / 5$, respectively.

\section{CONCLUDING REMARKS, FUTURE PROSPECTS, AND UNSOLVED PROBLEMS}

\section{A. General considerations}

In this Section, we try to outline the directions along which the field of shot noise in mesoscopic systems has been developing, to point out the unsolved problems which are, in our opinion, important, and to guess how the field will further develop. A formal summary can be found at the end of the Section.

Prior to the development of the theory and the experiments on shot noise in mesoscopic physics, there already existed a considerable amount of knowledge in condensed matter physics, electrical engineering, and especially optics. Both theory and experiments are available in these fields, and the results are well established. Similarly in mesoscopic physics, there exists a fruitful interaction between theory and experiment. However, presently there are many theoretical predictions concerning shot noise, not only extensions of the existing theories, but which really address new sub-fields, which are not yet tested experimentally. Below we give a short list of these predictions. Like every list, the choice reflects very much our taste, and we do not imply that the predictions not included in this list are of minor importance.
- 1/4-suppression of shot noise in chaotic cavities;

- Multi-terminal effects probing statistics (exchange Hanbury Brown - Twiss (HBT) effect; shot noise at tunnel microscope tips; HBT effect with FQHE edge states);

- Frequency dependent noise beyond NyquistJohnson (noise measurements which would reveal the inner energy scales of mesoscopic systems); current fluctuations induced into gates or other nearby mesoscopic conductors;

- Shot noise of clean NS interfaces; mesoscopic nature of positive cross-correlations in hybrid structures;

- Shot noise in high magnetic fields at the half-filled Landau level;

- Shot noise in hybrid magnetic structures.

The theory, in our opinion, is generally well developed for most of the field and adequately covers it. However, a number of problems persist: For instance, there is no clear understanding under which conditions the crosscorrelations in multi-terminal hybrid structures may be positive. Recent work 179 suggests that it is only a mesoscopic quantum contribution which is positive, but that to leading order the correlations will be negative as in normal conductors. Considerably more work is required on the frequency dependence of shot noise and on strongly correlated systems. The former (Section III) offers the opportunities to probe the inner energy scales and collective relaxation times of the mesoscopic systems; only a few results are presently available. As for the strongly correlated systems (including possibly unconventional superconductors), this may become (and is already becoming) one of the mainstreams of mesoscopic physics; since even the dc shot noise measurements provide valuable information about the charge and statistics of quasiparticles, we expect a lot of theoretical developments in this direction concerning the shot noise. Some of the unsolved problems in this field may be found directly in Section VII, one of the most fascinating being the possibility of probing the quasiparticle statistics in multi-terminal noise measurements with FQHE edge states.

One more possible development, which we did not mention in the main body of this Review, concerns shot noise far from equilibrium under conditions when the $I-V$ characteristics are non-linear. The situation with non-linear problems resembles very much the frequency dependent ones: Current conservation and gauge invariance are not automatically guaranteed, and interactions must be taken into account to ensure these properties (for a discussion, see e.g. Ref. 153). Though in the cases which we cited in the Review the non-linear results seem to be credible, it is still desirable to have a 
gauge-invariant general theory valid for arbitrary nonlinear $I-V$ characteristics. It is also desirable to gain insight and develop estimates of the range of applicability of the usual theories. Recently Wei et al 334 derived a gauge invariant expression for shot noise in the weakly non-linear regime, expressing it through functional derivatives of the Lindhard function with respect to local potential fields. They apply the results to the resonant tunneling diode. Wei et al 334 also discuss the limit in which the tunneling rates may be assumed to be energy independent. Apparently, the theory of Wei et al does not treat the effect of fluctuations of the potential inside the sample, which may be an important source of noise. Furthermore, Green and Das 335 337 proposed a classical theory of shot noise, based on a direct solution of kinetic equations. They discuss the possibility to detect interaction effects in the cross-over region from thermal to shot noise. It is, however, yet to be shown what results this approach yields in the linear regime and whether it reproduces, for instance, the 1/3-suppression of shot noise in metallic diffusive wires. An application of both of these approaches to specific systems is highly desirable.

What we have mentioned above, concerns the development of a field inside mesoscopic physics. We expect, however, that interesting connections will occur across the boundaries of different fields. An immediate application which can be imagined is the shot noise of photons and phonons. Actually, noise is much better studied in optics than in condensed matter physics (see e.g. the review article [338]), and, as we have just mentioned previously, the theory of shot noise in mesoscopic physics borrowed many ideas from quantum optics. At the same time, mesoscopic physics gained a huge experience in dealing, for instance, with disordered and chaotic systems. Recently a "back-flow" of this experience to quantum optics started. This concerns photonic noise for the transmission through (disordered) waveguides, or due to the radiation of random lasers or cavities of chaotic shape. In particular, the waveguides and cavity may be absorbing or amplifying, which adds new features as compared with condensed matter physics 77 . For references, we cite a recent review by Patra and Beenakker [339]. Possibly, in the future other textbook problems of mesoscopic physics will also find their analogies in quantum optics. Phonons are less easy to manipulate with, but, in principle, one can also imagine the same class of problems for them. Generally, shot noise accompanies the propagation of any type of (quasi)-particles; as the last

\footnotetext{
${ }^{77}$ One has to take, of course, also into account the differences between photon and electron measurements: Apart from the evident Bose versus Fermi and neutral versus charged particles, there are many more. For example, the photon measurement is accompanied by a removal of a photon from the device, while the total electron number is always conserved.
}

example, we mention plasma waves.

\section{B. Summary for a lazy or impatient reader}

Below is a summary of this Review. Though we encourage the reader to work through the whole text (and then she or he does not need this summary), we understand that certain readers are too lazy or too impatient to do this. For such readers we prepare this summary which permits to acquire some information on shot noise in a very short time-span. We only include in this summary the statements which in our opinion are the most important.

- Shot noise occurs in a transport state and is due to fluctuations in the occupation number of states caused by (i) thermal random initial fluctuations; (ii) the random nature of quantum-mechanical transmission/reflection (partition noise), which, in turn, is a consequence of the discreteness of the charge of the particles. The actual noise is a combination of both of these microscopic sources and typically these sources cannot be separated.

- Shot noise provides information about the kinetics of the transport state: In particular, it can be used to obtain information on transmission channels beyond that contained in the conductance. In two-terminal systems, zero-frequency shot noise for non-interacting electrons is suppressed in comparison with the Poisson value $S=2 e\langle I\rangle$.

- For quantum wells the noise suppression is $F=$ $S / 2 e\langle I\rangle=\left(\Gamma_{L}^{2}+\Gamma_{R}^{2}\right) /\left(\Gamma_{L}+\Gamma_{R}\right)^{2}$. The suppression is universal for metallic diffusive wires $(F=1 / 3)$ and chaotic cavities $(F=1 / 4)$.

- Far from equilibrium, in the vicinity of instability points the shot noise can exceed the Poisson value.

- In the limit of low transmission, the shot noise is Poissonian and measures the charge of transmitting particles. In particular, for normal metal - superconductor interfaces this charge equals $2 e$, whereas in SNS systems it is greatly enhanced due to multiple Andreev reflections. In the limit of low reflection, the shot noise may be understood as Poissonian noise of reflected particles; in this way, the charge of quasiparticles in the fractional quantum Hall effect is measured.

- For carriers with Fermi statistics, in multi-terminal systems the zero-frequency correlations of currents at different terminals are always negative. For Bose statistics, they may under certain circumstances become positive. These cross-correlations may be used to probe the statistics of quasiparticles. 
- The ensemble averaged shot noise may be described both quantum-mechanically (scattering approach; Green's function technique) and classically (master equation; Langevin and Boltzmann-Langevin approach; minimal correlation approach). Where they can be compared, classical and quantummechanical descriptions provide the same results. Classical methods, of course, fail to describe genuinely quantum phenomena like e.g. the quantum Hall effect.

- As a function of frequency, the noise crosses over from the shot noise to the equilibrium noise $S \propto$ $|\omega|$. This is only valid when the frequency is low as compared with the inner energy scales of the system and inverse times of the collective response. For higher frequencies, the noise is sensitive to all these scales. However, the current conservation for frequency dependent noise is not automatically provided, and generally is not achieved in noninteracting systems.

- Inelastic scattering may enhance or suppress noise, depending on its nature. In particular, in macroscopic systems shot noise is always suppressed down to zero by inelastic (usually, electron-phonon) scattering. When interactions are strong, shot noise is usually Poissonian, like in the Coulomb blockade plateau regime.

- We expect that the future development of the field of the shot noise will be mainly along the following directions: Within the field of mesoscopic electrical systems: (i) experimental developments; (ii) frequency dependence; and (iii) shot noise in strongly correlated systems; and more generally (iv) shot noise in disordered and chaotic quantum optical systems, shot noise measurements of phonons (and, possibly, of other quasiparticles).

\section{ACKNOWLEDGEMENTS}

We have profited from discussions and a number of specific comments by Pascal Cedraschi, Thomas Gramespacher, and Andrew M. Martin. Some parts of this Review were written at the Aspen Center for Physics (Y. M. B); at the Max-Planck-Institut für Physik Komplexer Systeme, Dresden (Y. M. B.); at the Centro Stefano Franscini, Ascona (Y. M. B. and M. B.); and at the Université de Montpellier II (Y. M. B.). We thank these institutions for hospitality and support. This work was supported by the Swiss National Science Foundation via the Nanoscience program.

\section{APPENDIX A: COUNTING STATISTICS AND OPTICAL ANALOGIES}

The question which naturally originates after consideration of the shot noise is the following: Can we obtain some information about the higher moments of the current? Since, as we have seen, the shot noise at zero frequency contains more information about the transmission channels than the average conductance, the studies of the higher moments may reveal even more information. Also, we have seen that in the classical theories of shot noise the distribution of the Langevin sources (elementary currents) is commonly assumed to be Gaussian, in order to provide the equivalence between the Langevin and Fokker-Planck equations [213]. An independent analysis of the higher moments of the current can reveal whether this equivalence in fact exists, and thus how credible the classical theory is.

A natural quantity to study is the $k$-th cumulant of the number of particles $n(t)$ which passed through the barrier during the time $t$ (which is assumed to be large). In terms of the current $I(t)$, this cumulant is expressed as

$$
\left\langle\left\langle n^{k}(t)\right\rangle\right\rangle=\frac{1}{e^{k}} \int_{0}^{t} d t_{1} \ldots d t_{k}\left\langle\left\langle I\left(t_{1}\right) \ldots I\left(t_{k}\right)\right\rangle\right\rangle,
$$

where $\langle\langle\ldots\rangle\rangle$ means the cumulant (irreducible part). For the following, we only consider the time-independent problems, i.e. the noise in the presence of a dc voltage. Then the first cumulant is $\langle\langle n(t)\rangle\rangle=\langle I\rangle t / e$, and the second one is expressed through the zero-temperature shot noise power,

$$
\left\langle\left\langle n^{2}(t)\right\rangle\right\rangle=\frac{S t}{2 e^{2}} .
$$

In particular, the ratio of $\left\langle\left\langle n^{2}(t)\right\rangle\right\rangle$ and $\langle\langle n(t)\rangle\rangle$ gives the Fano factor. The cumulants with $k>2$ in Eq. (A1) contain additional information about the statistics of current. Thus, if the distribution of the transmitted charge is Poissonian, all the cumulants have the same value; for the Gaussian distribution all the cumulants with $k>2$ vanish.

The general expression for the cumulants of the number of transmitted particles was obtained by Lee, Levitov, and Yakovets 340], who followed the earlier paper ${ }^{8}$ by Levitov and Lesovik 341. We only give the results for zero temperature. Consider first one channel with the transmission probability $T$. The probability that $m$ particles pass through this channel during the time $t$ is given by Bernoulli distribution, as found by Shimizu and Sakaki 343] and Levitov and Lesovik [341],

$$
P_{m}(t)=C_{N}^{m} T^{m}(1-T)^{N-m}, \quad m \leq N,
$$

\footnotetext{
${ }^{78}$ The paper 341] corrects Ref. 342.
} 
where $N(t)$ is the "number of attempts", on average given by $\langle N\rangle=e V t / 2 \pi \hbar$. Actually, $N(t)$ fluctuates, but in the long-time limit $N \gg 1$ these fluctuations are insignificant [341, and therefore $N$ in Eq. (A2) must be understood as the average value. The expression (A2) gives the probability that out of $N$ attempts $m$ particles go through (with the probability $T$ ) and $N-m$ others are reflected back.

The next step is to define the characteristic function,

$$
\chi(\lambda)=\sum_{m=0}^{N} P_{m} \exp (i m \lambda)=\left[T e^{i \lambda}+1-T\right]^{N} .
$$

If we have several independent 79 channels, the characteristic function is multiplicative,

$$
\chi(\lambda)=\prod_{j}\left[T_{j} e^{i \lambda}+1-T_{j}\right]^{N},
$$

where the product is taken over all the transmission channels. The coefficients in the series expansion of $\ln \chi$ are the cumulants that we are looking for,

$$
\ln \chi_{\lambda}=\sum_{k=1}^{\infty} \frac{(i \lambda)^{k}}{k !}\left\langle\left\langle n^{k}(t)\right\rangle\right\rangle
$$

and Lee, Levitov, and Yakovets 340] obtain in this way the explicit expression,

$$
\left\langle\left\langle n^{k}(t)\right\rangle\right\rangle=\left.N \sum_{j}\left[T(1-T) \frac{d}{d T}\right]^{k-1} T\right|_{T=T_{j}} .
$$

We see from Eq. (A6) that $\langle\langle n(t)\rangle\rangle=N \sum T_{j}$, and $\left\langle\left\langle n^{2}(t)\right\rangle\right\rangle=N \sum T_{j}\left(1-T_{j}\right)$, which are the results for the average current and the zero frequency shot noise power. However, higher cumulants do not vanish at all. Though they generally cannot be calculated in a closed form, the distributions are studied for many systems, and we give a brief overview below.

For the tunnel barrier, when $T_{j} \ll 1$ for any $j$, all the cumulants are equal, $\left\langle\left\langle n^{k}(t)\right\rangle\right\rangle=N \sum T_{j}$, and thus the distribution of the transmitted charge is Poissonian.

De Jong 344 analyzes the counting statistics for double-barrier structures using the concept of the distribution function for the transmission probabilities. For the symmetric case $\left(\Gamma_{L}=\Gamma_{R}=\Gamma\right)$ he finds $\langle\ln \chi\rangle=$ $2 N_{\perp} \Gamma t(\exp (i \lambda / 2)-1)$, and explicitly for the cumulants $\left\langle\left\langle n^{k}(t)\right\rangle\right\rangle=N_{\perp} \Gamma t / 2^{k-1}$. Here $N_{\perp}$ is the number of transverse channels at the Fermi surface. The cumulants decrease exponentially with $k$, and thus the statistics are closer to Gaussian than to Poissonian. De Jong 344 was

\footnotetext{
${ }^{79}$ In the sense that we can diagonalize the matrix $t^{\dagger} t$ and define the transmission eigenvalues.
}

also able to obtain the same results classically, starting from the master equation.

The case of metallic diffusive wires is considered by Lee, Levitov, and Yakovets [340, and subsequently by Nazarov [345]. They find that the disorder-averaged logarithm of the characteristic function is 80

$$
\langle\ln \chi(\lambda)\rangle=\frac{\langle I\rangle t}{e} \operatorname{arcsinh}^{2} \sqrt{e^{i \lambda}-1} .
$$

The expressions for the cumulants cannot be found in a closed form. As expected, from Eq. (A7) we obtain $\left\langle\left\langle n^{2}\right\rangle\right\rangle=\langle I\rangle t / 3 e$, in agreement with the fact that the Fano factor is $1 / 3$. For the following cumulants one gets, for instance, $\left\langle\left\langle n^{3}\right\rangle\right\rangle=\langle I\rangle t / 15 e$ and $\left\langle\left\langle n^{4}\right\rangle\right\rangle=$ $-\langle I\rangle t / 105 e$. For high $k$ the cumulants $\left\langle\left\langle n^{k}\right\rangle\right\rangle$ behave as $(k-1) ! /\left((2 \pi)^{k} k^{1 / 2}\right)$, i.e. they diverge! Moreover, Lee, Levitov, and Yakovets 340] evaluate the sampleto-sample fluctuations of the cumulants, and find that for the high-order cumulants these fluctuations become stronger than the cumulants themselves. Thus, the far tails of the charge distributions are strongly affected by disorder. Nazarov 345] generalizes the approach to treat weak localization corrections.

For the transmission through the symmetric chaotic cavity, Ref. 347 finds

$$
\langle\ln \chi(\lambda)\rangle=\frac{4\langle I\rangle t}{e} \ln \frac{e^{i \lambda / 2}+1}{2},
$$

with the explicit expression for the cumulants

$$
\left\langle\left\langle n^{2 l}\right\rangle\right\rangle=\frac{\langle I\rangle t}{e} \frac{2^{2 l}-1}{2^{2 l-1} l} B_{2 l},
$$

and $\left\langle\left\langle n^{2 l+1}\right\rangle\right\rangle=0(l \geq 1)$. Here $B_{k}$ are the Bernoulli numbers $\left(B_{2}=1 / 6, B_{4}=-1 / 30\right)$. Indeed, for the second cumulant we obtain $\left\langle\left\langle n^{2}\right\rangle\right\rangle=\langle I\rangle t / 4 e$, in accordance with the $1 / 4$-shot noise suppression in symmetric chaotic cavities. Eq. (A9) can be also obtained classically [347], using the generalization of the minimal correlation approach.

Muzykantskii and Khmelnitskii 169 investigate the counting statistics for the NS interface and find

$$
\chi(\lambda)=\prod_{j}\left[T_{A j} e^{2 i \lambda}+1-T_{A j}\right]^{N}, \quad T_{A j} \equiv \frac{2 T_{j}^{2}}{\left(2-T_{j}\right)^{2}} .
$$

\footnotetext{
${ }^{80}$ The characteristic function is not self-averaging; the expansion of $\ln \langle\chi(\lambda)\rangle$ yields different expression for the cumulants, as found by Muttalib and Chen [346]. As Levitov, Lee, and Yakovets 340 argue, the correct quantity to average is $\ln \chi(\lambda)$, rather than $\chi(\lambda)$, since it is linearly related to the cumulants of the transmitted charge.
} 
It is clearly seen from the comparison with Eq. (A4) that the particles responsible for transport have effective charge $2 e$.

Other important developments include the generalization to the multi-terminal (Levitov and Lesovik [341]) and time-dependent (Ivanov and Levitov [348]; Levitov, Lee, and Lesovik [349]; Ivanov, Lee, and Levitov 350]) problems, and numerical investigation of the counting statistics for the non-degenerate ballistic conductors (Bulashenko et al 267]).

Thus, the counting statistics certainly reveal more information about the transport properties of conductors than is contained in either the conductance or the second order shot noise. The drawback is that it is not quite clear how these statistics can be measured. A proposal, due to Levitov, Lee, and Lesovik [349], is to use the spin-1/2 galvanometer, precessing in the magnetic field created by the transmission current. The idea is to measure the charge transmitted during a certain time interval through the evolution of the spin precession angle. However, the time dependent transport is a collective phenomenon (Section IIII), and thus the theory of such an effect must include electron-electron interactions. In addition, this type of experiments is not easy to realize.

On the other hand, measurements of photon numbers are routinely performed in quantum optics. In this field concern with counting statistics has already a long history. However, typical mesoscopic aspects - disorder, weak localization, chaotic cavities - and effects particular to optics, like absorption and amplification, make the counting statistics of photons a promising tool of research. Some of these aspects (relating to disorder and chaos, where the random matrix theory may be applied), have been recently investigated by Beenakker [351]; however, there are still many unsolved problems.

\section{APPENDIX B: SPIN EFFECTS AND ENTANGLEMENT}

A notion which mesoscopic physics recently borrowed from quantum optics is entanglement. States are called entangled, if they cannot be written simply as a product of wave functions. For our purpose, we will adopt the following definition. Imagine that we have two leads, 1 and 3, which serve as sources of electrons. The entangled states are defined as the following two-particle states described in terms of the creation operators,

$$
| \pm\rangle=\frac{1}{\sqrt{2}}\left(\hat{a}_{3 \downarrow}^{\dagger}\left(E_{2}\right) \hat{a}_{1 \uparrow}^{\dagger}\left(E_{1}\right) \pm \hat{a}_{3 \uparrow}^{\dagger}\left(E_{2}\right) \hat{a}_{1 \downarrow}^{\dagger}\left(E_{1}\right)\right)|0\rangle,
$$

where $\hat{a}_{\alpha \sigma}^{\dagger}(E)$ is the operator which creates an electron with the energy $E$ and the spin projection $\sigma$ in the source $\alpha$. The state corresponding to the lower sign in Eq. (B1) is the spin singlet with the symmetric orbital part of the wave function, while the upper sign describes a triplet (antisymmetric) state.
Such entangled states are very important in the field of quantum computation. Condensed matter systems are full of entangled states: there is hardly a system for which the ground state can be expressed simply in terms of a product of wave functions. The key problem is to find ways in which entangled states can be generated and manipulated in a controlled way. Optical experiments on noise have reached a sophisticated stage since there exist optical sources of entangled states (the production of twin photon-pairs through down conversion). It would be highly desirable to have an electronic equivalent of the optical source and to analyze to what extent such experiments can be carried out in electrical conductors [352,108. An example of such source is a p-n junction which permits the generation of an electron-hole pair and the subsequent separation of the particles. The disadvantage of such an entangled state is that the electron and hole must be kept apart at all times. Similarly, a Cooper pair entering a normal conductor, represents an entangled state. But in the normal conductor it is described as an electron-hole excitation and there is to our knowledge no deterministic way to separate the electron and the hole. To date most proposals in condensed matter related to quantum computation consider entangled states in closed systems.

Theoretically, entanglement opens a number of interesting opportunities. One of the questions is: Provided we were able to prepare entangled states, how do we know the states are really entangled? Since we deal with twoparticle states, it is clear that entanglement can only be measured in the experiments which are genuinely twoparticle. Burkard, Loss, and Sukhorukov 353,354 investigate the multi-terminal noise. Indeed, add to the structure two more reservoirs (electron detectors) 2 and 4 , and imagine that there is no reflection back to the sources (the geometry of the exchange HBT experiment, Fig. 15(b), with the additional "entangler" creating the states (B1)). The system acts as a three-terminal device, with an input of entangled electrons and measuring the current-current correlation at the two detectors.

Since the shot noise is produced by the motion of the electron charge, it is plausible that the noise measurements are in fact sensitive to the symmetry of the orbital part of the wave function, and not to the whole wave function. Thus the noise power seen at a single contact is expected to be enhanced for the singlet state (symmetric orbital part) and suppressed for the triplet state (anti-symmetric orbital part). It is easy to quantify these considerations by repeating the calculation of Section II in the basis of entangled states (B1). Assuming that the system is of finite size, so that the set of energies $E$ is discrete, and the incoming stream of entangled electrons is noiseless, Burkard, Loss, and Sukhorukov [353] obtained the following result, $\left\langle I_{2}\right\rangle=\left\langle I_{4}\right\rangle, S_{22}=S_{44}=-S_{24}$, with

$$
S_{22}=2 e T(1-T)\left(1 \mp \delta_{E_{1}, E_{2}}\right) .
$$

Thus, indeed, the shot noise is suppressed for the triplet state and enhanced for the singlet state, provided the 
electrons are taken at (exactly) the same energy. For the singlet state this suppression is an indication of the entanglement, since there are no other singlet states. One can also construct the triplet states which are not entangled,

$$
|\uparrow \uparrow\rangle=\hat{a}_{3 \uparrow}^{\dagger}\left(E_{2}\right) \hat{a}_{1 \uparrow}^{\dagger}\left(E_{1}\right)|0\rangle,
$$

and an analogous state with spins down. These states, as shown by Burkard, Loss, and Sukhorukov [353], produce the same noise as the entangled triplet state. Thus, the noise suppression in this geometry is not a signature of the entanglement.

Another proposal, due to Loss and Sukhorukov [303], is that the entangled states prepared in the double quantum dot can be probed by the Aharonov-Bohm transport experiments. The shot noise is Poissonian in this set-up, and both current and shot noise are sensitive to the symmetry of the orbital wave function.

More generally, one can also ask what happens if one can operate with spin-polarized currents separately. (Again, presently no means are known to do this). Burkard, Loss, and Sukhorukov 353] considered a transport in a two-terminal conductor where the chemical potentials are different for different spin projections. In particular, if $V_{\uparrow}=-V_{\downarrow}$, the total average current is zero (the spin-polarized currents compensate each other). Shot noise, however, exists, and may be used as a means to detect the motion of electrons in this situation.

\section{APPENDIX C: NOISE INDUCED BY THERMAL TRANSPORT}

Sukhorukov and Loss 114 consider shot noise in metallic diffusive conductors in the situation when there is no voltage applied between the reservoirs, and the transport is induced by the difference of temperatures. To this end, they generalize the Boltzmann-Langevin approach to the case of non-uniform temperature. For the simplest situation of a two-terminal conductor, when one of the reservoirs is kept at zero temperature, and the other at the temperature $T$, their result reads

$$
S=\frac{4}{3}(1+\ln 2) G k_{B} T
$$

for the purely elastic scattering, with $G$ being the Drude conductance. This shows, in particular, that the noise induced by thermal transport is also universal - the ratio of the shot noise power to the thermal current does not depend on the details of the sample. This is an experiment that would be interesting to realize.

Another prospective problem concerning the noise induced by the non-uniform temperature, is that the applied temperature gradient would cause not only the transport of electrons, but also transport of phonons. Thus, in this kind of experiments one can study shot noise (and, possibly, also counting statistics) of phonons.
This really looks very promising, and, to our knowledge, by now has never been discussed.

[1] M. J. M. de Jong and C. W. J. Beenakker, in: Mesoscopic Electron Transport, ed. by L. L. Sohn, L. P. Kouwenhoven, and G. Schön, NATO ASI Series E, Vol. 345 (Kluwer Academic Publishing, Dordrecht, 1997) 225.

[2] Th. Martin, in: Coulomb and Interference Effects in Small Electronic Structures, ed by D. C. Glattli, M. Sanquer, and J. Trân Thanh Vân (Editions Frontières, Gifsur-Yvette, 1994) 405.

[3] Sh. Kogan, Electronic noise and Fluctuations in Solids (Cambridge University Press, Cambridge, 1996).

[4] Y. Imry, Introduction to Mesoscopic Physics (Oxford University Press, Oxford, 1997).

[5] W. Schottky, Ann. Phys. (Leipzig) 57 (1918) 541.

[6] A. van der Ziel, Noise (Chapman and Hall, London, 1955).

[7] R. Hanbury Brown and R. Q. Twiss, Nature 177 (1956) 27.

[8] R. Loudon, in: Disorder in Condensed Matter Physics, ed. by J. A. Blackman and J. Taguena (Clarendon Press, Oxford, 1991) 441.

[9] M. Büttiker, Phys. Rev. B 46 (1992) 12485.

[10] A. Prêtre, H. Thomas, and M. Büttiker, Phys. Rev. B 54 (1996) 8130.

[11] L. D. Landau and E. M. Lifshits, Statistical Physics, Pt.1 (Butterwort-Heinemann, Oxford, 1980), §118.

[12] M. Henny, S. Oberholzer, C. Strunk, T. Heinzel, K. Ensslin, M. Holland, and C. Schönenberger, Science 284 (1999) 296.

[13] G. B. Lesovik and R. Loosen, Z. Phys. B 91 (1993) 531.

[14] I. O. Kulik and A. N. Omel'yanchuk, Fiz. Nizk. Temp. 10 (1984) 305 [Sov. J. Low Temp. Phys. 10 (1984) 158].

[15] V. A. Khlus, Zh. Éksp. Teor. Fiz. 93 (1987) 2179 [Sov. Phys. JETP 66 (1987) 1243];

[16] G. B. Lesovik, Pis'ma Zh. Éksp. Teor. Fiz. 49 (1989) 513 [JETP Lett. 49 (1989) 592].

[17] B. Yurke and G. P. Kochanski, Phys. Rev. B 41 (1990) 8184 .

[18] M. Büttiker, Phys. Rev. Lett. 65 (1990) 2901.

[19] M. Büttiker, Physica B 175 (1991) 199.

[20] R. Landauer and Th. Martin, Physica B 175 (1991) 167; ibid 182 (1992) 288 (E).

[21] Th. Martin and R. Landauer, Phys. Rev. B 45 (1992) 1742.

[22] M. H. Pedersen and M. Büttiker (unpublished).

[23] A. I. Larkin and Yu. N. Ovchinnikov, Zh. Éksp. Teor. Fiz. 53 (1967) 2159 [Sov. Phys. JETP 26 (1968) 1219].

[24] A. J. Dahm, A. Denenstein, D. N. Langenberg, W. H. Parker, D. Rogovin, and D. J. Scalapino, Phys. Rev. Lett. 22 (1969) 1416.

[25] D. Rogovin and D. J. Scalapino, Physica 55 (1971) 399.

[26] D. Rogovin and D. J. Scalapino, Ann. Phys. (N. Y.) 86 
(1974) 1.

[27] G. Schön, Phys. Rev. B 32 (1985) 4469.

[28] H. Lee and L. S. Levitov, Phys. Rev. B 53 (1996) 7383.

[29] S. T. Liu and A. van der Ziel, Physica 37 (1967) 241.

[30] H. Birk, M. J. M. de Jong, and C. Schönenberger, Phys. Rev. Lett. 75 (1995) 1610.

[31] B. J. van Wees, H. van Houten, C. W. J. Beenakker, J. G. Williamson, L. P. Kouwenhoven, D. van der Marel, and C. T. Foxon, Phys. Rev. Lett. 60 (1988) 848.

[32] D. A. Wharam, T. J. Thornton, R. Newbury, M. Pepper, H. Ahmed, J. E. F. Frost, D. G. Hasko, D. C. Peacock, D. A. Ritchie, and G. A. C. Jones, J. Phys. C 21 (1988) L209.

[33] L. I. Glazman, G. B. Lesovik, D. E. Khmel'nitskii, and R. I. Shekhter, Pis'ma Zh. Éksp. Teor. Fiz. 48 (1988) 218 [JETP Lett. 48 (1988) 238].

[34] M. Büttiker, Phys. Rev. B 41 (1990) 7906.

[35] A. G. Scherbakov, E. N. Bogachek, and U. Landman, Phys. Rev. B 57 (1998) 6654.

[36] L. Y. Chen and S. C. Ying, Mod. Phys. Lett. B 9 (1995) 573.

[37] I. A. Larkin and M. Reznikov, in: Proc. of 23rd Int. Conf. on the Physics of Semiconductors, ed. by M. Scheffler and R. Zimmermann (World Scientific, Singapore, 1996) 1565.

[38] A. I. Akimenko, A. B. Verkin, and I. K. Yanson, J. Low Temp. Phys. 54 (1984) 247. See their Fig. 9.

[39] Y. P. Li, D. C. Tsui, J. J. Heremans, J. A. Simmons, and G. W. Weimann, Appl. Phys. Lett. 57 (1990) 774.

[40] C. Dekker, A. J. Scholten, F. Liefrink, R. Eppenga, H. van Houten, and C. T. Foxon, Phys. Rev. Lett. 66 (1991) 2148.

[41] F. Liefrink, A. J. Scholten, C. Dekker, R. Eppenga, H. van Houten, and C. T. Foxon, Physica B 175 (1991) 213.

[42] M. I. Reznikov, M. Heiblum, H. Shtrikman, and D. Mahalu, Phys. Rev. Lett. 75 (1995) 3340.

[43] A. Kumar, L. Saminadayar, D. C. Glattli, Y. Jin, and B. Etienne, Phys. Rev. Lett. 76 (1996) 2778.

[44] H. E. van den Brom and J. M. van Ruitenbeek, Phys. Rev. Lett. 82 (1999) 1526.

[45] J. Bürki and C. A. Stafford, Phys. Rev. lett. 83 (1999) 3342.

[46] A. D. Stone and P. A. Lee, Phys. Rev. Lett. 54 (1985) 1196.

[47] M. Büttiker, IBM J. Res. Develop. 32 (1988) 63.

[48] L. Y. Chen and C. S. Ting, Phys. Rev. B 43 (1991) 4534.

[49] A. Reklaitis and L. Reggiani, J. Appl. Phys. 82 (1997) 3161.

[50] A. Reklaitis and L. Reggiani, Phys. Stat. Solidi (b) 204 (1997) 459.

[51] D. V. Averin, J. Appl. Phys. 73 (1993) 2593.

[52] R. Tsu and L. Esaki, Appl. Phys. Lett. 22 (1973) 562.

[53] L. L. Chang, L. Esaki, and R. Tsu, Appl. Phys. Lett. 24 (1974) 593.

[54] J. H. Davies, P. Hyldgaard, S. Hershfield, and J. W. Wilkins, Phys. Rev. B 46 (1992) 9620.

[55] J. A. Melsen and C. W. J. Beenakker, Physica B 203 (1994) 219.
[56] V. I. Melnikov, Phys. Lett. A 198 (1995) 459.

[57] E. Runge, Phys. Rev. B 47 (1993) 2003.

[58] Ø. Lund Bø and Yu. Galperin, J. Phys. Cond. Matter 8 (1996) 3033.

[59] Ø. Lund Bø and Yu. Galperin, Phys. Rev. B 55 (1997) 1696.

[60] S.-J. Xiong, J. Appl. Phys. 78 (1995) 6079.

[61] Yu. V. Nazarov and J. J. R. Struben, Phys. Rev. B 53 (1996) 15466.

[62] A. I. Larkin and K. A. Matveev, Zh. Éksp. Teor. Fiz. 93 (1987) 1030 [Sov. Phys. JETP 66 (1987) 580].

[63] L. I. Glazman and K. A. Matveev, Pis'ma Zh. Éksp. Teor. Fiz. 48 (1988) 403 [JETP Lett. 48 (1988) 445].

[64] Y. P. Li, A. Zaslavsky, D. C. Tsui, M. Santos, and M. Shayegan, Phys. Rev. B 41 (1990) 8388.

[65] T. G. van de Roer, H. C. Heyker, J. J. M. Kwaspen, H. P. Joosten, and M. Henini, Electron. Lett. 27 (1991) 2158.

[66] P. Ciambrone, M. Macucci, G. Iannaccone, B. Pellegrini, M. Lazzarino, L. Sorba, and F. Beltram, Electron. Lett. 31 (1995) 503.

[67] H. C. Liu, J. Li, G. C. Aers, C. R. Leavens, M. Buchanan, and Z. R. Wasilewski, Phys. Rev. B 51 (1995) 5116.

[68] A. Przadka, K. J. Webb, D. B. Janes, H. C. Liu, and Z. R. Wasilewski, Appl. Phys. Lett. 71 (1997) 530.

[69] S.-T. Yau, H. B. Sun, P. J. Edwards, and P. Lynam, Phys. Rev. B 55 (1997) 12880.

[70] O. N. Dorokhov, Solid State Commun. 51 (1984) 381.

[71] Y. Imry, Europhys. Lett. 1 (1986) 249.

[72] C. W. J. Beenakker, Rev. Mod. Phys. 69 (1997) 731.

[73] A. D. Stone, P. A. Mello, K. A. Muttalib, and J.L. Pichard, in: Mesoscopic Phenomena in Solids, ed. by B. L. Altshuler, P. A. Lee, and R. A. Webb (NorthHolland, Amsterdam, 1991) 369.

[74] C. W. J. Beenakker and M. Büttiker, Phys. Rev. B 46 (1992) 1889.

[75] K. E. Nagaev, Phys. Lett. A 169 (1992) 103.

[76] B. L. Altshuler, L. S. Levitov, and A. Yu. Yakovets, Pis'ma Zh. Éksp. Teor. Fiz. 59 (1994) 821 [JETP Lett. 59 (1994) 857].

[77] Yu. V. Nazarov, Phys. Rev. Lett. 73 (1994) 134.

[78] Ya. M. Blanter and M. Büttiker, Phys. Rev. B 56 (1997) 2127.

[79] F. Liefrink, J. I. Dijkhuis, M. J. M. de Jong, L. W. Molenkamp, and H. van Houten, Phys. Rev. B 49 (1994) 14066.

[80] A. H. Steinbach, J. M. Martinis, and M. H. Devoret, Phys. Rev. Lett. 76 (1996) 3806.

[81] M. Henny, S. Oberholzer, C. Strunk, and C. Schönenberger, Phys. Rev. B 59 (1999) 2871.

[82] M. J. M. de Jong and C. W. J. Beenakker, Phys. Rev. B 46 (1992) 13400.

[83] A. M. S. Macêdo, Phys. Rev. B 49 (1994) 1858.

[84] A. M. S. Macêdo and J. T. Chalker, Phys. Rev. B 49 (1994) 4695.

[85] A. M. S. Macêdo, Phys. Rev. Lett. 79 (1997) 5098.

[86] C. Mudry, P. W. Brouwer, and A. Furusaki, Phys. Rev. B 59 (1999) 13221. 
[87] C. Mudry, P. W. Brouwer, and A. Furusaki (unpublished).

[88] R. Gade, Nucl. Phys. B 398 (1993) 499.

[89] M. J. M. de Jong and C. W. J. Beenakker, Phys. Rev. B 51 (1995) 16867.

[90] M. J. M. de Jong and C. W. J. Beenakker, Physica A 230 (1996) 219.

[91] R. C. Liu, P. Eastman, and Y. Yamamoto, Solid State Commun. 102 (1997) 785.

[92] C. W. J. Beenakker and J. A. Melsen, Phys. Rev. B 50 (1994) 2450.

[93] K. M. Schep and G. E. W. Bauer, Phys. Rev. B 56 (1997) 15860.

[94] I. L. Aleiner and L. I. Glazman, Phys. Rev. B 57 (1998) 9608.

[95] P. W. Brouwer and I. L. Aleiner, Phys. Rev. Lett. 82 (1999) 390.

[96] H. U. Baranger and P. Mello, Phys. Rev. Lett. 73 (1994) 142.

[97] R. A. Jalabert, J.-L. Pichard, and C. W. J. Beenakker, Europhys. Lett. 27 (1994) 255.

[98] M. H. Pedersen, S. A. van Langen, and M. Büttiker, Phys. Rev. B 57 (1998) 1838.

$[99]$ Ya. M. Blanter and E. V. Sukhorukov, condmat/9904448.

[100] Yu. V. Nazarov, in: Quantum Dynamics of Submicron Strustures, ed. by H. A. Cerdeira, B. Kramer, and G. Schön, NATO ASI Series E, Vol. 291 (Kluwer Academic Publishing, Dordrecht, 1995) 687.

[101] P. W. Brouwer and C. W. J. Beenakker, J. Math. Phys. 37 (1996) 4904.

[102] M. Büttiker, Phys. Rev. B 38 (1988) 9375.

[103] S. Komiyama and H. Hirai, Phys. Rev. B 54 (1996) 2067.

[104] A. J. Kil, R. J. J. Zijlstra, P. M. Koenraad, J. A. Pals, and J. P. André, Solid State Commun. 60 (1986) 831.

[105] B. E. Kane, D. C. Tsui, and G. Weimann, Surf. Sci. 196 (1988) 183.

[106] S. Washburn, R. J. Haug, K. Y. Lee, and J. M. Hong, Phys. Rev. B 44 (1991) 3875.

[107] W. D. Oliver, J. Kim, R. C. Liu, and Y. Yamamoto, Science 284 (1999) 299.

[108] M. Büttiker, Science 284 (1999) 275.

[109] Ç. Kurdak, C.-J. Chen, D. C. Tsui, J. P. Lu, M. Shayegan, S. Parihar, and S. A. Lyon, Surf. Sci. 361/362 (1996) 705.

[110] T. Gramespacher and M. Büttiker, Phys. Rev. Lett. 81 (1998) 2763.

[111] T. Gramespacher and M. Büttiker, Phys. Rev. B 60 (1999) 2375.

[112] M. Büttiker, Phys. Rev. Lett. 68 (1992) 843.

[113] E. V. Sukhorukov and D. Loss, Phys. Rev. Lett. 80 (1998) 4959.

[114] E. V. Sukhorukov and D. Loss, Phys. Rev. B 59 (1999) 13054.

[115] S. A. van Langen and M. Büttiker, Phys. Rev. B 56 (1997) R1680.

[116] Ya. M. Blanter, S. A. van Langen, and M. Büttiker, Usp. Fiz. Nauk 168 (1998) 159 [Physics-Uspekhi 41 (1998) 149].
[117] R. C. Liu, Y. Yamamoto, and S. Tarucha, Physica B 249-251 (1998) 152.

[118] R. C. Liu, B. Odom, Y. Yamamoto, and S. Tarucha, Nature 391 (1998) 263.

[119] B. L. Altshuler, A. G. Aronov, and B. Z. Spivak, Pis'ma Zh. Éksp. Teor. Fiz. 33 (1981) 101 [JETP Lett. 33 (1981) 94].

[120] Y. Gefen, Y. Imry, and M. Ya. Azbel, Phys. Rev. Lett. 52 (1984) 129.

[121] M. Büttiker, Y. Imry, and M. Ya. Azbel, Phys. Rev. A 30 (1984) 1982.

[122] M. A. Davidovich and E. V. Anda, Phys. Rev. B 50 (1994) 15453.

[123] G. Iannaccone, M. Macucci, and B. Pellegrini, Phys. Rev. B 56 (1997) 12104.

[124] M. Büttiker, in: SQUID'85. Superconducting Quantum Interference Devices and their Applications, ed. by H. D. Hahlbohm and H. Lübbig (Walter de Gruyter, Berlin, 1985), 529 .

[125] M. Büttiker, Phys. Rev. B 33 (1986) 3020.

[126] J. L. D. D'Amato and H. M. Pastawski, Phys. Rev. B 41 (1990) 7411.

[127] F. Gagel and K. Maschke, Phys. Rev. B 52 (1995) 2013.

[128] G. Müller, D. Weiss, S. Koch, K. von Klitzing, H. Nickel, W. Schlapp, and R. Lösch, Phys. Rev. B 42 (1990) 7633.

[129] R. C. Liu and Y. Yamamoto, Phys. Rev. B 49 (1994) 10520.

[130] R. C. Liu and Y. Yamamoto, Phys. Rev. B 50 (1994) 17411.

[131] M. Büttiker, in: Noise in Physical Systems and $1 / f$ Fluctuations, ed. by V. Bareikis and R. Katilius (World Scientific, Singapore, 1995) 35.

[132] P. W. Brouwer and C. W. J. Beenakker, Phys. Rev. B 55 (1997) 4695.

[133] A. Shimizu and M. Ueda, Phys. Rev. Lett. 69 (1992) 1403.

[134] L. Y. Chen and C. S. Ting, Phys. Rev. B 46 (1992) 4714.

[135] M. Büttiker, in Quantum Dynamics of Submicron Strustures, ed. by H. A. Cerdeira, B. Kramer, and G. Schön, NATO ASI Series E, Vol. 291 (Kluwer Academic Publishing, Dordrecht, 1995) 657.

[136] M. Büttiker, J. Phys. Cond. Matter 5 (1993) 9361.

[137] I. L. Aleiner and A. I. Larkin, Phys. Rev. B 54 (1996) 14423.

[138] P. W. Brouwer and M. Büttiker, Europhys. Lett. 37 (1997) 441.

[139] Ya. M. Blanter and M. Büttiker, Europhys. Lett. 42 (1998) 535.

[140] Y. Naveh, D. V. Averin, and K. K. Likharev, Phys. Rev. Lett. 79 (1997) 3482.

[141] K. E. Nagaev, Phys. Rev. B 57 (1998) 4628.

[142] Y. Naveh, D. V. Averin, and K. K. Likharev, Phys. Rev. B 59 (1999) 2848.

[143] K. E. Nagaev, Phys. Rev. B 58 (1998) R7513.

[144] Y. Naveh, Phys. Rev. B 58 (1998) R13387.

[145] Y. Naveh, cond-mat/9806348.

[146] M. Büttiker, Phys. Rev. B 45 (1992) 3807.

[147] E. Ben-Jacob, E. Mottola, and G. Schön, Phys. Rev. Lett. 51 (1983) 2064. 
[148] S.-R. E. Yang, Solid State Commun. 81 (1992) 375.

[149] M. Ueda and A. Shimizu, J. Phys. Soc. Jap. 62 (1993) 2994.

[150] R. J. Schoelkopf, P. J. Burke, A. A. Kozhevnikov, D. E. Prober, and M. J. Rooks, Phys. Rev. Lett. 78 (1997) 3370.

[151] D. Zheng, Z. D. Wang, and Y. Liu, Phys. Lett. A 208 (1995) 375.

[152] G. B. Lesovik and L. S. Levitov, Phys. Rev. Lett. 72 (1994) 538.

[153] M. H. Pedersen and M. Büttiker, Phys. Rev. B 58 (1998) 12993.

[154] R. J. Schoelkopf, A. A. Kozhevnikov, D. E. Prober, and M. J. Rooks, Phys. Rev. Lett. 80 (1998) 2437.

[155] Y. Levinson and P. Wölfle, Phys. Rev. Lett. 83 (1999) 1399.

[156] M. Büttiker, in: Noise in Physical Systems and $1 / f$ Fluctuations, ed. by P. H. Handel and A. Chung, AIP Conf. Proc. No. 285 (AIP, New York, 1993) 3.

[157] M. Büttiker, H. Thomas, and A. Prêtre, Phys. Lett. A 180 (1993) 364.

[158] T. Kuhn, L. Reggiani, and L. Varani, Superlatt. Microstruct. 11 (1992) 205.

[159] T. Kuhn, L. Reggiani, and L. Varani, Semicond. Sci. Technol. 7 (1992) B495.

[160] R. Dashen, S. H. Ma, and H. J. Bernstein, Phys. Rev. 187 (1969) 345.

[161] F. Kassubek, C. A. Stafford, and H. Grabert, Phys. Rev. B 59 (1999) 7560.

[162] V. A. Gopar, P. A. Mello, and M. Büttiker, Phys. Rev. Lett. 77 (1996) 3005.

[163] C. W. J. Beenakker (private communication, 1999).

[164] M. Büttiker, J. Korean Phys. Soc. 34 (1999) 121 condmat/9902054).

[165] Ya. M. Blanter, F. W. J. Hekking, and M. Büttiker, Phys. Rev. Lett. 81 (1998) 1925.

[166] M. Büttiker and A. M. Martin, cond-mat/9902320.

[167] G. E. Blonder, M. Tinkham, and T. M. Klapwijk, Phys. Rev. B 25 (1982) 4515.

[168] S. Datta, P. F. Bagwell, and M. P. Anantram, Phys. Low-Dim Struct. 3 (1996) 1.

[169] B. A. Muzykantskii and D. E. Khmelnitskii, Phys. Rev. B 50 (1994) 3982.

[170] M. J. M. de Jong and C. W. J. Beenakker, Phys. Rev. B 49 (1994) 16070.

[171] Th. Martin, Phys. Lett. A 220 (1996) 137.

[172] S. V. Naidenov and V. A. Khlus, Fiz. Nizk. Temp. 21 (1995) 594 [Low Temp. Phys. 21 (1995) 462].

[173] A. L. Fauchère, G. B. Lesovik, and G. Blatter, Phys. Rev. B 58 (1998) 11177.

[174] M. P. Anantram and S. Datta, Phys. Rev. B 53 (1996) 16390.

[175] J.-X. Zhu and C. S. Ting, Phys. Rev. B 59 (1999) R14165.

[176] G. B. Lesovik, Th. Martin, and J. Torrès, condmat/9902278

[177] A. M. Martin, T. Gramespacher, and M. Büttiker, Phys. Rev. B 60 (1999) Nov. 1 cond-mat/9907240.

[178] J. Torrès and Th. Martin, cond-mat/9906012.

[179] T. Gramespacher and M. Büttiker, cond-mat/9908469.
[180] A. N. Vystavkin and M. A. Tarasov, Pis'ma Zh. Tekh. Phys. 9 (1983) 869 [Sov. Tech. Phys. Lett. 9 (1983) 373].

[181] X. Jehl, P. Payet-Burin, C. Baraduc, R. Calemczuk, and M. Sanquer, Phys. Rev. Lett. 83 (1999) 1660.

[182] K. K. Likharev, Rev. Mod. Phys. 51 (1979) 101.

[183] H. Kanter and F. L. Vernon, Jr., Appl. Phys. Lett. 16 (1970) 115.

[184] H. Kanter and F. L. Vernon, Jr., Phys. Rev. Lett. 25 (1970) 588.

[185] M. J. Stephen, Phys. Rev. Lett. 21 (1968) 1629.

[186] M. J. Stephen, Phys. Rev. 182 (1969) 531.

[187] K. K. Likharev and V. K. Semenov, Pis'ma Zh. Éksp. Teor. Fiz. 15 (1972) 625 [JETP Lett. 15 (1972) 442].

[188] A. G. Aronov and R. Katilius, Zh. Éksp. Teor. Fiz. 68 (1975) 2208 [Sov. Phys. JETP 41 (1975) 1106].

[189] P. S. Landa and N. D. Tarankova, Fiz. Nizk. Temp. 3 (1977) 32 [Sov. J. Low Temp. Phys. 3 (1977) 15].

[190] A. B. Zorin, Fiz. Nizk. Temp. 7 (1981) 709 [Sov. J. Low Temp. Phys. 7 (1981) 346].

[191] G. B. Lesovik, Pis'ma Zh. Éksp. Teor. Fiz. 60 (1994) 806 [JETP Lett. 60 (1994) 820].

[192] W. H. Parker, B. N. Taylor, and D. N. Langenberg, Phys. Rev. Lett. 18 (1967) 287.

[193] G. Vernet and R. Adde, Appl. Phys. Lett. 19 (1971) 195.

[194] R. K. Kirschman and J. E. Mercereau, Phys. Lett. A 35 (1971) 177.

[195] M. Tarasov, D. Golubev, V. Shumeiko, Z. Ivanov, E. Stepantsov, O. Harnak, and T. Claeson, Pis'ma Zh. Éksp. Teor. Fiz. 68 (1998) 426 [JETP Lett. 68 (1998) 454].

[196] I. O. Kulik, Zh. Éskp. Teor. Fiz. 57 (1969) 1745 [Sov. Phys. JETP 30 (1970) 944].

[197] A. Furusaki and M. Tsukada, Physica B 165 \& 166 (1990) 967.

[198] S. N. Artemenko, A. F. Volkov, and A. V. Zaitsev, Zh. Éksp. Teor. Fiz. 76 (1979) 1816 [Sov. Phys. JETP 49 (1979) 924].

[199] M. Büttiker, Phys. Rev. B 32, 1846 (1985).

[200] M. Büttiker, Ann. N.Y. Acad. Sciences 480 (1986) 194.

[201] D. Averin and H. T. Imam, Phys. Rev. Lett. 76 (1996) 3814.

[202] A. Martín-Rodero, A. Levy Yeyati, and F. J. GarcíaVidal, Phys. Rev. B 53 (1996) R8891.

[203] J. C. Cuevas, A. Martín-Rodero, and A. Levy Yeyati, Phys. Rev. Lett. 82 (1999) 4086.

[204] T. M. Klapwijk, G. E. Blonder, and M. Tinkham, Physica B 109 \& 110 (1992) 1657.

[205] D. Averin, Physica B 227241 (1996).

[206] Y. Naveh and D. V. Averin, Phys. Rev. Lett. 82 (1999) 4090.

[207] E. V. Bezuglyi, E. N. Bratus', V. S. Shumeiko, and G. Wendin, Phys. Rev. Lett. 83 (1999) 2050.

[208] J. P. Hessling, V. S. Shumeiko, Yu. M. Galperin, and G. Wendin, Europhys. Lett. 34 (1996) 49.

[209] Y. Misaki, A. Saito, and K. Hamasaki, Jpn. J. Appl. Phys. Pt. 135 (1996) 1190.

[210] Y. Misaki, A. Saito, S. Anezaki, and K. Hamasaki, IEEE Trans. Appl. Supercond. 7 (1997) 2438.

[211] P. Dieleman, H. G. Bukkems, T. M. Klapwijk, 
M. Schicke, and K. H. Gundlach, Phys. Rev. Lett. 79 (1997) 3486 .

[212] T. Hoss, C. Strunk, T. Nussbaumer, R. Huber, U. Staufer, and C. Schönenberger, cond-mat/9901129.

[213] N. G. van Kampen, Stochastic Processes in Physics and Chemistry (North-Holland, Amsterdam, 1992).

[214] K. E. Nagaev, cond-mat/9908193.

[215] R. Landauer, Phys. Rev. B 47 (1993) 16427.

[216] L. Y. Chen, G. Levine, J. Yang, and C. S. Ting, Mod. Phys. Lett. 5 (1991) 315.

[217] E. Barkai, R. S. Eisenberg, and Z. Schuss, Phys. Rev. E 54 (1996) 1161.

[218] N. G. van Kampen, Physica A 244 (1997) 414.

[219] M. E. Raikh (private communication, 1991).

[220] A. Imamoḡlu and Y. Yamamoto, Phys. Rev. Lett. 70 (1993) 3327.

[221] L. Y. Chen, Phys. Rev. B 48 (1993) 4914.

[222] H.-O. Müller, A. Hädicke, U. Hanke, and K. A. Chao, Z. Phys. B 102 (1997) 557.

[223] H. B. Sun and G. J. Milburn, Superlatt. Microstruct. 23 (1998) 883.

[224] H. B. Sun and G. J. Milburn, Phys. Rev. B 59 (1999) 10748.

[225] G. J. Milburn, cond-mat/9905360.

[226] Ya. M. Blanter and M. Büttiker, in: Quantum Physics at Mesoscopic Scale, ed. by D. C. Glattli, M. Sanquer, and J. Trân Thanh Vân (Editions Frontières, Gif-surYvette, 1999)(to be published).

[227] L. Y. Chen, Mod. Phys. Lett. 7 (1993) 1677.

[228] G. Iannaccone, M. Macucci, and B. Pellegrini, Phys. Rev. B 55 (1997) 4539.

[229] J. H. Davies, J. Carlos Egues, and J. W. Wilkins, Phys. Rev. B 52 (1995) 11259.

[230] Y. Isawa, N. Matsubara, and T. Ohuti, Solid-State Electron. 42 (1998) 1515.

[231] E. R. Brown, IEEE Trans. Electron Devices 39 (1992) 2686.

[232] V. V. Kuznetsov, E. E. Mendez, J. D. Bruno, and J. T. Pham, Phys. Rev. B 58 (1998) R10159.

[233] J. Han and F. S. Barnes, IEEE Trans. Electron Devices 38 (1991) 237.

[234] M. A. Alam and A. N. Khondker, IEEE Trans. Electron Devices 39 (1992) 2184.

[235] H. Sheng and S.-J. Chua, J. Phys. D 27 (1994) 137.

[236] M. M. Jahan and A. F. M. Anwar, Solid-State Electron. 38 (1995) 429.

[237] A. Sugimura, Solid State Commun. 87 (1993) 461.

[238] J. Carlos Egues, S. Hershfield, and J. W. Wilkins, Phys. Rev. B 49 (1994) 13517.

[239] A. Levy Yeyati, F. Flores, and E. V. Anda, Phys. Rev. B 47 (1993) 10543.

[240] G. Iannaccone, G. Lombardi, M. Macucci, and B. Pellegrini, Phys. Rev. Lett. 80 (1998) 1054.

[241] Ya. M. Blanter and M. Büttiker, Phys. Rev. B 59 (1999) 10217.

[242] V. J. Goldman, D. C. Tsui, and J. E. Cunningham, Phys. Rev. Lett. 58 (1987) 1256.

[243] P. Hänggi and H. Thomas, Phys. Repts. 88 (1982) 207.

[244] Sh. M. Kogan and A. Ya. Shul'man, Zh. Éksp. Teor. Fiz. 56 (1969) 862 [Sov. Phys. JETP 29 (1969) 467].
[245] A. Schmid, Z. Phys. 271 (1974) 251.

[246] B. L. Altshuler and A. G. Aronov, in: Electron-electron Interactions in Disordered Systems, ed. by A. L. Efros and M. Pollak (North-Holland, Amsterdam, 1985), 1.

[247] Ya. M. Blanter, Phys. Rev. B 54, 12807 (1996).

[248] K. E. Nagaev, Phys. Rev. B 52 (1995) 4740.

[249] V. I. Kozub and A. M. Rudin, Phys. Rev. B 52 (1995) 7853.

[250] V. I. Kozub and A. M. Rudin, Pis'ma Zh. Éksp. Teor. Fiz. 62 (1995) 45 [JETP Lett. 62 (1995) 49].

[251] B. L. Altshuler, A. G. Aronov, and D. E. Khmelnitsky, J. Phys. C 15 (1982) 7367.

[252] F. von Oppen and A. Stern, Phys. Rev. Lett. 79 (1997) 1114.

[253] V. L. Gurevich and A. M. Rudin, Pis'ma Zh. Éksp. Teor. Fiz. 62 (1995) 13 [JETP Lett. 62 (1995) 12].

[254] V. L. Gurevich and A. M. Rudin, Phys. Rev. B 53 (1996) 10078.

[255] A. Schmid, Z. Phys. B 259 (1973) 421.

[256] Y. Naveh, D. V. Averin, and K. K. Likharev, Phys. Rev. B 58 (1998) 15371.

[257] M. Turlakov, cond-mat/9908298.

[258] T. González, C. González, J. Mateos, D. Pardo, L. Reggiani, O. M. Bulashenko, and J. M. Rubí, Phys. Rev. Lett. 80 (1998) 2901.

[259] T. González, J. Mateos, D. Pardo, O. M. Bulashenko, and L. Reggiani, Phys. Rev. B 60 (1999) 2670.

[260] C. W. J. Beenakker, Phys. Rev. Lett. 82 (1999) 2761.

[261] K. E. Nagaev, Phys. Rev. Lett. 83 (1999) 1267.

[262] H. Schomerus, E. G. Mishchenko, and C. W. J. Beenakker, Phys. Rev. B 60 (1999) 5839.

[263] H. Schomerus, E. G. Mishchenko, and C. W. J. Beenakker, cond-mat/9907027.

[264] O. M. Bulashenko, J. M. Rubi, and V. A. Kochelap, cond-mat/9906179.

[265] T. González, J. Mateos, D. Pardo, L. Varani, and L. Reggiani, cond-mat/9910125.

[266] T. González, O. M. Bulashenko, J. Mateos, D. Pardo, and L. Reggiani, Phys. Rev. B 56 (1997) 6424.

[267] O. M. Bulashenko, J. Mateos, D. Pardo, T. González, L. Reggiani, and J. M. Rubí, Phys. Rev. B 57 (1998) 1366 .

[268] T. González, O. M. Bulashenko, J. Mateos, D. Pardo, L. Reggiani, and J. M. Rubí, Semicond. Sci. Technol. 12 (1997) 1053.

[269] Y. Naveh, A. N. Korotkov, and K. K. Likharev, Phys. Rev. B 60 (1999) R2169.

[270] V. Tripathi and D. E. Khmelnitskii, Phys. Rev. B 58 (1998) 1122.

[271] Ya. M. Blanter, A. D. Mirlin, and B. A. Muzykantskii, Phys. Rev. Lett. 80 (1998) 4161.

[272] E. Cuevas, E. Louis, and J. A. Vergés, Phys. Rev. Lett. 77 (1996) 1970.

[273] E. Louis, E. Cuevas, J. A. Vergés, and M. Ortuño, Phys. Rev. B 56 (1997) 2120.

[274] V. I. Okulov and V. V. Ustinov, Fiz. Nizk. Temp. 5 (1979) 213 [Sov. J. Low Temp. Phys. 5 (1979) 101].

[275] S. V. Gantsevich, V. L. Gurevich, and R. Katilius, Riv. Nuovo Cimento 2, No. 6 (1979).

[276] M. Lax, Rev. Mod. Phys. 32 (1960) 25. 
[277] C. W. J. Beenakker and H. van Houten, Phys. Rev. B 43 (1991) 12066.

[278] D. V. Averin and K. K. Likharev, in: Mesoscopic Phenomena in Solids, ed. by B. L. Altshuler, P. A. Lee, and R. A. Webb (North-Holland, Amsterdam, 1991) 173.

[279] M. A. Belogolovskii and L. G. Levin, Pis'ma Zh. Tekh. Phys. 17 (1991) 84 [Sov. Tech. Phys. Lett. 17 (1991) 311].

[280] A. N. Korotkov, D. V. Averin, K. K. Likharev, and S. A. Vasenko, in: Single-Electron Tunneling and Mesoscopic Devices, ed. by H. Koch and H. Lübbig, Springer Series in Electronics and Photonics, Vol. 31 (SpringerVerlag, Berlin, 1992) 45.

[281] A. N. Korotkov, Phys. Rev. B 49 (1994) 10381.

[282] A. N. Korotkov, Europhys. Lett. 43 (1998) 343.

[283] S. Hershfield, J. H. Davies, P. Hyldgaard, C. J. Stanton, and J. W. Wilkins, Phys. Rev. B 47 (1993) 1967.

[284] K.-M. Hung and G. Y. Wu, Phys. Rev. B 48 (1993) 14687.

[285] Yu. M. Halperin, U. Hanke, K. A. Chao, and N. Zou, Mod. Phys. Lett. B 7 (1993) 1159.

[286] U. Hanke, Yu. M. Galperin, K. A. Chao, and N. Zou, Phys. Rev. B 48 (1993) 17209.

[287] U. Hanke, Yu. M. Galperin, and K. A. Chao, Phys. Rev. B 50 (1994) 1595.

[288] E. V. Anda and A. Latgé, Phys. Rev. B 50 (1994) 8559.

[289] Z. Wang, M. Iwanaga, and T. Miyoshi, Jpn. J. Appl. Phys. Pt. 137 (1998) 5894.

[290] W. Krech, A. Hädicke, and H.-O. Müller, Int. J. Mod. Phys. B 6 (1992) 3555.

[291] W. Krech and H.-O. Müller, Z. Phys. B 91 (1993) 423.

[292] K. A. Matsuoka and K. K. Likharev, Phys. Rev. B 57 (1998) 15613.

[293] A. N. Korotkov, D. V. Averin, and K. K. Likharev, Phys. Rev. B 49 (1994) 1915.

[294] A. N. Korotkov, Phys. Rev. B 50 (1994) 17674.

[295] D. V. Averin and Yu. V. Nazarov, Phys. Rev. Lett. 69 (1992) 1993.

[296] F. W. J. Hekking, L. I. Glazman, K. A. Matveev, and R. I. Shekhter, Phys. Rev. Lett. 70 (1993) 4138.

[297] W. Krech and H.-O. Müller, Mod. Phys. Lett. B 8 (1994) 605.

[298] U. Hanke, M. Gisselfält, Yu. Galperin, M. Jonson, R. I. Shekhter, and K. A. Chao, Phys. Rev. B 50 (1994) 1953.

[299] U. Hanke, Yu. Galperin, K. A. Chao, M. Gisselfält, M. Jonson, and R. I. Shekhter, Phys. Rev. B 51 (1995) 9084.

[300] B. R. Bułka, J. Martinek, G. Michałek, and J. Barnaś, cond-mat/9904341.

[301] Ya. M. Blanter (unpublished).

[302] E. R. Nowak, M. B. Weissman, and S. S. P. Parkin, Appl. Phys. Lett. 74 (1999) 600

[303] D. Loss and E. V. Sukhorukov, cond-mat/9907129.

[304] L. I. Glazman and M. E. Raikh, Pis'ma Zh. Eksp. Teor. Fiz. 47 (1988) 378 [JETP Lett. 47 (1988) 452].

[305] T. K. Ng and P. A. Lee, Phys. Rev. Lett. 61 (1988) 1768.

[306] S. Hershfield, J. H. Davies, and J. W. Wilkins, Phys. Rev. B 46 (1992) 7046.
[307] S. Hershfield, Phys. Rev. B 46 (1992) 7061.

[308] F. Yamaguchi and K. Kawamura, J. Phys. Soc. Jap. 63 (1994) 1258.

[309] F. Yamaguchi and K. Kawamura, Physica B 227 (1996) 116.

[310] G.-H. Ding and T.-K. Ng, Phys. Rev. B 56 (1997) R15521.

[311] A. Schiller and S. Hershfield, Phys. Rev. B 58 (1998) 14978.

[312] M. P. A. Fisher and L. I. Glazman, in: Mesoscopic Electron Transport, ed. by L. L. Sohn, L. P. Kouwenhoven, and G. Schön, NATO ASI Series E, Vol. 345 (Kluwer Academic Publishing, Dordrecht, 1997) 331.

[313] C. de C. Chamon, D. E. Freed, and X. G. Wen, Phys. Rev. B 53 (1996) 4033.

[314] C. L. Kane and M. P. A. Fisher, Phys. Rev. Lett. 72 (1994) 724.

[315] A. Auerbach, Phys. Rev. Lett. 80 (1998) 817.

[316] P. Fendley, A. W. W. Ludwig, and H. Saleur, Phys. Rev. Lett. 75 (1995) 2196.

[317] P. Fendley and H. Saleur, Phys. Rev. B 54 (1996) 10845.

[318] U. Weiss, Solid State Commun. 100 (1996) 281.

[319] I. Safi, Phys. Rev. B 56 (1997) R12691.

[320] N. P. Sandler, C. de C. Chamon, and E. Fradkin, Phys. Rev. B 59 (1999) 12521.

[321] V. V. Ponomarenko and N. Nagaosa, Solid State Commun. 110 (1999) 321.

[322] C. de C. Chamon, D. E. Freed, and X. G. Wen, Phys. Rev. B 51 (1995) 2363.

[323] F. Lesage and H. Saleur, Nucl. Phys. B 490 (1997) 543.

[324] F. Lesage and H. Saleur, Nucl. Phys. B 493 (1997) 613.

[325] C. Chamon and D. E. Freed, Phys. Rev. B 60 (1999) 1848.

[326] K.-I. Imura and K. Nomura, Europhys. Lett. 47 (1999) 83.

[327] S. B. Isakov, Th. Martin, and S. Ouvry, Phys. Rev. Lett. 83 (1999) 580.

[328] L. Saminadayar, D. C. Glattli, Y. Jin, and B. Etienne, Phys. Rev. Lett. 79 (1997) 2526.

[329] R. de-Picciotto, M. Reznikov, M. Heiblum, V. Umansky, G. Bunin, and D. Mahalu, Nature 389 (1997) 162.

[330] M. Reznikov, R. de Picciotto, T. G. Griffiths, M. Heiblum, and V. Umansky, Nature 399 (1999) 238.

[331] F. von Oppen, Phys. Rev. B 56 (1997) 9674.

[332] G. Kirczenow, Phys. Rev. B 58 (1998) 1457.

[333] R. de Picciotto, cond-mat/9802221.

[334] Y. Wei, B. Wang, J. Wang, and H. Guo, condmat/9902306.

[335] F. Green and M. P. Das, cond-mat/9809339.

[336] F. Green and M. P. Das, cond-mat/9905086.

[337] M. P. Das and F. Green, cond-mat/9910183.

[338] C. H. Henry and R. F. Kazarinov, Rev. Mod. Phys. 68 (1996) 801.

[339] C. W. J. Beenakker and M. Patra, Mod. Phys. Lett. B 13 (1999) 337.

[340] H. Lee, L. S. Levitov, and A. Yu. Yakovets, Phys. Rev. B 51 (1995) 4079.

[341] L. S. Levitov and G. B. Lesovik, Pis'ma Zh. Éksp. Teor. Fiz. 58 (1993) 225 [JETP Lett. 58 (1993) 230].

[342] L. S. Levitov and G. B. Lesovik, Pis'ma Zh. Éksp. Teor. 
Fiz. 55 (1992) 534 [JETP Lett. 55 (1992) 555].

[343] A. Shimizu and H. Sakaki, Phys. Rev. B 44 (1991) 13136.

[344] M. J. M. de Jong, Phys. Rev. B 54 (1996) 8144.

[345] Yu. V. Nazarov, Ann. Phys. (Leipzig) 8 (1999) 507.

[346] K. A. Muttalib and Y. Chen, Int. J. Mod. Phys. B 10 (1996) 1999.

[347] Ya. M. Blanter, H. Schomerus, and C. W. J. Beenakker (unpublished).

[348] D. A. Ivanov and L. S. Levitov, Pis'ma Zh. Éksp. Teor. Fiz. 58 (1993) 450 [JETP Lett. 58 (1993) 461].

[349] L. S. Levitov, H. Lee, and G. B. Lesovik, J. Math. Phys. 37 (1996) 4845.

[350] D. A. Ivanov, H. W. Lee, and L. S. Levitov, Phys. Rev. B 56 (1997) 6839.

[351] C. W. J. Beenakker, in: Diffuse Waves in Complex Media, ed. by J.-P. Fouque, NATO ASI Series C, Vol. 531 (Kluwer Academic Publishing, Dordrecht, 1999) 137.

[352] D. P. DiVincenzo and D. Loss, J. of Magnetism and Magnetic Materials 200 (1999) 202 (condmat/9901137).

353] G. Burkard, D. Loss, and E. V. Sukhorukov, condmat/9906071.

354] E. V. Sukhorukov, D. Loss, and G. Burkard, condmat/9909348. 